
. 

(1) $M$

$8 b+17$.

i 


\title{
THE LIASSIC THERAPSID OLIGOKYPHUS
}

BY

WALTER GEORG KÜHNE

\author{
WITH TWELVE STEREOSCOPIC PLATES \\ $A N D$ \\ SIXTY-SIX FIGURES IN THE TEXT
}

\section{LONDON \\ PRINTED BY ORDER OF \\ THE TRUSTEES OF THE BRITISH MUSEUM \\ I956}


$\ominus$ 


\section{CONTENTS}

PAGE

PREFACE

LIST OF PLATES

LIST OF FIGURES IN THE TEXT

INTRODUCTION

GEOLOGY

BIOSTRATONOMY

SKULL .

Premaxillary .

Septomaxillary

Maxillary

Lacrimal

Jugal .

Dentary

Splenial .

Identification of Articular and Quadrate

Orientation of the Articular Complex and the Quadrate

Description of the Articular Complex

Quadrate

Squamosal

Brain-case

Exoccipital

Supraoccipital

Basioccipital

Periotic

Internal View of Brain-case

Inner Ear

Stapes

Basisphenoid

Brain

Epipterygoid

Pterygoid

Vomer

Palatine

Dermal Bones of the Brain-case and Roof of the Skull

Reconstruction of the Skull

DENTITION

Upper Cheek Teeth

Lower Cheek Teeth

Succession and Wear of Teeth

Description of Incisors of Large and Small Adult Forms

Function of the Dentition

TAXONOMY . 
VERTEBRAL COLUMN

P.AGE

Post-axial Vertebral Column . $\quad$. . . . . . . . . . . . . . . . IOI

RIBS

PRESTERNUM

II 2

STERNUM

II 3

SHOULDER GIRDLE

Clavicle

Scapulo-coracoid

FORE LIMB

Humerus

Ulna

Radius.

PELVIC GIRDLE .

Ilium

Ischium

Pubis

HIND LIMB :

Femur .

Tibia

Fibula .

Tarsus [and Carpus]

Calcaneum

Astragalus

Metapodials

Phalanges

Claw 


\section{PREFACE}

Nearly a century ago Charles Moore investigated the Rhaetic deposits filling a fissure in the Carboniferous Limestone at Holwell in Somerset, and at the Bath meeting of the British Association in 1864 he exhibited twenty-nine mammalian teeth as the most important result of his labour in washing and sorting three cartloads of material. He estimated that the clay had yielded him altogether " probably one million specimens " including in addition to marine invertebrates " relics of nine genera of reptiles ... and fifteen genera of fishes"; of Acrodus alone he produced 70,00o teeth. Yet in spite of this shining example the Mesozoic fissure-fillings of the Mendips were subsequently strangely neglected, until in I938 a young German palaeontologist, Walter Kühne, arrived in this country with the express intention of collecting early mammals from such deposits. Scarcely deflected from his ultimate goal by the "gale of Europe" which sprang up in 1939 and after violent storms landed him in the calm haven of an internment camp, Dr. Kühne was able to continue his studies with the help of additional material collected under the auspices of the Geological Department of the British Museum by the late Dorothea Bate and Mr. L. E. Parsons. After the end of the war Dr. Kühne completed his investigations in the Zoological Department of University College, working in conjunction with the Geological Department of the British Museum, where the greater part of his collection is now housed.

The present monograph is some measure of his success as a collector and his skill as an investigator, while the conclusion that the creature whose remains he has assembled in such abundance is strictly speaking a reptile rather than a mammal does not in the least detract from its scientific interest and importance. The author returned to Germany before the text and illustrations were complete, and much of the work of final preparation for the press has fallen on Dr. W. E. Swinton and Mr. F. M. Wonnacott. We trust that the process of editing has admitted no untoward discrepancies or errors.

March I955.

W. N. EDWARDS, Keeper of Geology.

Circumstances have delayed the publication of this monograph until after Mr. Edwards' retirement from the Keepership of the Department. His sucessor can only echo, with diffidence, the hope expressed in the last sentence of Mr. Edwards' preface.

June 1956.

ERROL I. WHITE, Keeper of Geology. 



\section{LIST OF PLATES}

(All are of Oligokyphus)

\section{PLATE}

I. Premaxillaries and maxillaries.

2. Maxillaries.

3. Jugals and Lacrimals.

4. Dentaries.

5. Dentary, basisphenoid, epipterygoid, articular and quadrate.

6. Squamosals.

7. Basioccipital, periotic and tabular.

8. Posterior half of skull and brain-case.

9. Periotic and basicranium.

Io. Epipterygoid, pterygoid, palatine and frontal.

II. Maxillaries, dentaries and cheek teeth.

I2. Cheek teeth; juvenile dentaries. 



\section{LIST OF FIGURES IN THE TEXT}

FIGURE

r. Oligokyphlis. Fractured ends of limb bones . . . . . . . . . . . . . . I4

Location and frequency of fractures in limb bones .

Dentary

Ir. Diademodon. Zygomatic arch .

r2. Bienotherium yunnanense. Sutures of the jugal . . . . . . . . . . . . 43

I3. Oligokyphus. Basicranium . . . . . . . . . . . . . . 47

14

Brain-case .

I5.

Brain cast and reconstruction

16.

Epipterygoid

I7.

Reconstruction of skull—dorsal view.

2r. Thrinaxodon liorhinus. Comparison of palate with Oligokyphus

22. Gomphognathus cf. mastacus. Comparison of palate with Oligokyphus 
FIGURE

PAGE

41. Diademodon. Left atlantal arch

42. Oligokyphus.

43.

Left atlantal arch

44.

Axis

Cervical and thoracic vertebrae

45.

Thoracic vertebrae.

46.

Sacral vertebrae

47.

Caudal vertebrae

48.

Caudal vertebrae

49.

Distal caudal vertebra

$50 . \quad$,

5 I. ,

$52 . \quad$

$53 . \quad$,

$54 . \quad$,

55 . "

$56 . \quad$,

$57 . \quad$,

$58 . \quad$,

59.

60.

6 I. ,

$62 . \quad$,

63.

$64 . \quad$,

$65 . \quad$,

66.

Rib outlines

Presternum

Right clavicle .

Right scapulo-coracoid

Right humerus

Right ulna

Right radius

Right half of pelvis

Left femur

Left tibia

Right fibula .

Left calcaneum

Left astragalus

Metacarpals

Metatarsals

Phalanges and claws

Reconstruction of skeleton 


\section{INTRODUGTION}

After the two catalogues on Mesozoic Mammalia by G. G. Simpson (I928, I929) had been written, incorporating all the material which was then known and recognized as mammalian, it became clear that any further advance in knowledge of these mammals could come only from new material. Investigations in the last ten years have helped to dispel the myth that Mesozoic Mammalia are extraordinarily rare and very difficult to find. It has been my experience when working in Britain that systematic and prolonged search is usually crowned with success, provided, of course, that such search is maintained in suitable areas and with suitable methods. This is not to say that the British Isles are predestined to contain a richer fauna of this kind than other areas of comparable size and structure, but it is certain that the possibilities of finding specimens here are very good indeed. The Rhaetic, Lias, Oolite, Purbeck and Wealden have yielded Mesozoic Mammalia, and future work will certainly result in still better finds from other horizons. It has been possible to show that at any locality which has yielded Mesozoic Mammalia a rich fauna, comprising many specimens, could be obtained. Hence, what is necessary is the discovery of new localities. It cannot be sufficiently emphasized that luck is not a factor of primary importance in finding such localities. It certainly played no part in the discovery of three localities of Mesozoic mammals in England. When fossils were collected by the slaters of Stonesfield, the few mammalian jaws "made their appearance" without being sought for. Charles Moore's work with the clay from the Holwell fissures led to the discovery of Microlestes ${ }^{1}$ simply because he worked with sufficient quantities to give Microlestes a chance to be found and his method was such that teeth were bound to turn up. The circumstances of the discovery of the mammal bed at Swanage by W. R. Brodie and C. Wilcox are unknown to me, but Owen (187I: 2I) states that the two discoverers were unaware of the mammalian nature of their finds.

Mesozoic mammals might be sought for either in sapropelic freshwater deposits or in the solution-cavities of older limestones which had been filled during the Mesozoic. The latter occurrences must be common, otherwise it would have been impossible for me in less than ten years to find two new localities in the very small area of England and Wales where the Lias transgresses on limestone. Indeed, once it is realized that bone accumulations are to be found in Mesozoic karst-regions it will not be long before our knowledge of mammals and other small terrestrial tetrapods of the Mesozoic is much more adequate. When the new localities have been found it will become evident that the Mesozoic Mammalia, though small and rather inconspicuous, were not rare in their time and constituted an essential component of the terrestrial faunas during the Rhaetic, Jurassic and Cretaceous.

One fact has more and more clearly emerged during work on the remains: that the tacitly accepted taxonomy of neontologists applied to the transition period, when some

1 The name Microlestes Plieninger 1847 being preoccupied by Microlestes Schmidt I846, Simpson replaced it in 1928 by Microcleptes. Since this too was preoccupied, by Microcleptes Newman I840, Simpson (I947: 497) coined the new name Haramiya. W. G. Kühne noted this but stated (I949:350, footnote) that he preferred to use the name Microlestes, not in defiance of nomenclatorial rules, but as "an application of commonsense and utility." 
reptiles were about to become mammals, does not lead to satisfactory results. All the forms evolving in this period, whether taxonomically " reptiles" or "mammals," are of vital interest in the early history of Mammalia. If one could see them in the flesh, osteological characters, which are unambiguous, would be depreciated considerably. Homeothermy, hairy coat, viviparity and diaphragmatic breathing evolved side by side with epiphyseal growth of bones, with a squamoso-dentary articulation of the jaw, with root-division in the cheek teeth and with a mammalian limb posture. As a logical consequence of the concept of a "transitional period," one should not find during this period mammals in the modern meaning of the word.

A host of strange and unexpected creatures lived then, representing the highest achievement of contemporary vertebrate evolution. Each of such genera sheds some light on the narrow phylogenetic track, represented by those forms which are the true mammalian ancestors, forms which were presumably rare and which evade most successfully the searching endeavours of any collector whose activities are not specially centred on them.

This work is devoted to a Tritylodontid; it deals exclusively with forms which are taxonomically reptiles. But this fact neither conflicts with an original intention to find and to work with Mesozoic Mammalia in England, nor does it imply that by allocating Tritylodon and its allies to the reptiles, their importance and their scientific value has in any degree decreased. The Tritylodontidae show admirably many characters which distinguish Mammalia from Cynodonts; their main interest lies in the fact that they bridge the wide gap in our knowledge which existed between well-known Cynodonts of the Middle Triassic and well-known Multituberculates of Palaeocene age and of the Recent Monotremes. The known Jurassic and Cretaceous Mammalia are not nearly so well represented and understood.

A long discussion about the mammalian or reptilian relationship of the Tritylodontidae and some other fossil animals of similar grade and age has thus ended with the result that this problem is now dismissed as essentially meaningless. What interests us is not the arbitrary allocation of the vertebrates in question to two preconceived classes -Mammalia or Reptilia - it is the retention of reptilian and the introduction of mammalian characters in animals which are "mammal-like" and which lived from Upper Triassic to Middle Jurassic times.

A number of papers written since Simpson's catalogues may be discussed here :

Broili \& Schröder (I936a) described new Tritylodon material from the Orange Free State. Unfortunately no lower dentitions were found. The designation of the specimen " I936 II 5 " as part of the " milk-dentition " I regard as unhappy, and it certainly implies more than is intended. A typical milk-dentition is only present in the majority of placental Mammalia-to which Tritylodon has no relation whatsoever. Furthermore, as will be shown, the Tritylodontid Oligokyphus did not even have tooth replacement. These Munich specimens will again be mentioned when dealing with the upper dentition of Oligokyphus and with taxonomy. Dietrich in 1937 described the ulna, Eoraetia siegerti, which has essential similarities with the ulna of Oligokyphus. Dietrich's excellent analysis will be considered later.

In 1937 and I938 Simpson, departing completely from his views of I928, separated the Tritylodontidae from the Multituberculates. This is the more remarkable as no material throwing new light on the problem of the systematic position of the Tritylodontidae had been found in the interval; it is an excellent instance of growing insight and profound 
knowledge approaching the truth step by step, without requiring fresh material evidence for an objective reinterpretation.

Butler's short and excellent paper (I939) on Tritylodon longaevus was the first occasion that this often misinterpreted specimen received justice so far as the dentition is concerned. The analysis of the cusp pattern is perfect, the drawing unimpeachable, and his conclusions will be discussed in the appropriate place. The paper reveals one slip of Simpson's, pointed out already by Broili \& Schröder (I936a) : the mid-row of the fourth cheek tooth has actually three cusps and not four.

Young in his first paper on Bienotherium (I940) states "that the ... described fossils belong to the Multituberculates is of course obvious." What Young meant was of course that Bienotherium is a Tritylodontid.

Watson's paper "On Permian and Triassic Tetrapods" (I942) puts in an admirable way all that could be said at that time about the Tritylodontids and about Bienotherium. His comparison of Bienotherium and Diademodon, implying a phylogenetic relationship between the Gomphodont reptiles and the Tritylodontidae, has stood the test of many detailed comparisons made possible by the new English material.

In my first paper (Kühne, I943) on the material of Oligokyphus now fully described here I reached, on the evidence of the dentary, conclusions similar to Watson's.

The merit of Bohlin's interesting paper (I946) is in the definite separation of the Tritylodontidae and Microlestidae from the Upper Jurassic Multituberculata, making the latter the last of mammalian orders to appear in the Jurassic. (For the Symmetrodonta see Kühne, I950a.)

Parrington (I947) defined the Sub-order Ictidosauria and made the Tritylodontidae a family of it. Finally, in I947, there appeared Young's latest and extensive paper on the Bienotherium material.

Considering Simpson's later departure from his opinion of I928, it would be most unfair to refute one by one his reasons for the mammalian nature of Tritylodon. Furthermore, his reservations (I928), which tend to make Tritylodon independent of the Upper Jurassic Multituberculata, are fully confirmed in the present account. Broom's verdict (I905), implying mammalian relationship for Tritylodon, was due to the a priori assumption that propalinal jaw movement is impossible with a primary jaw articulation, but, as Professor B. Peyer has pointed out to the writer, even Sphenodon moves its jaw in a longitudinal direction.

Unless a fossil skull can be compared with appropriate material only a little earlier or later in age, great caution is necessary when assessing its relationship. If a fossil skull is incomplete, still more caution is necessary, as unexpected combinations of characters are often found. If such incomplete fossil material is of an age where-on good evidence-we can expect a transition to take place (in our case, the transition from mammal-like reptiles to mammals), it is almost impossible to arrive at a final solution regarding the systematic position of the fossil.

Finally: a priori considerations are not helpful in determining the systematic position of such fossils. In the present case it appears that propalinal jaw movement is possible with a primary jaw-articulation (cf. Broom, I905), and that multiple roots of the cheek teeth can occur with a primary jaw articulation (cf. Simpson, I928, when dealing with the systematic position of the Microlestidae). 
For more than ten years work has been done on Oligokyphus from " Mendip I4 " and many ideas considered important when formulated have been superseded as better specimens have been found. It has been the author's practice when dealing with the reasons for identifying a certain skull bone, to give, where possible, evidence from articulation, though articulated fragments are rare.

The Text-figures of single bones are always based on all the evidence available, often obtained from many specimens. The stereoscopic plates will, it is hoped, speak for themselves. A stereoscope is, however, a necessity; without it, a reader will gather little information from the plates.

Since the discovery of Oligokyphus at "Mendip I4" in August I939 more than 2,000 specimens have been prepared, all the outlines of restored skeletal elements have been made and most of the photographic work done. Apart from the war years, when field work was impossible, each summer saw the author and his wife at the locality, and every season yielded some specimens which improved the osteological material.

My thanks are due to a number of people who contributed directly or indirectly to the successful completion of this work : Roads Reconstruction (I934) Ltd., the leaseholders of Windsor Hill Quarry, permitted the collection of matrix. Mr. W. N. Edwards arranged a collecting trip to Windsor Hill Quarry in I940 during the height of the war; the late Dorothea Bate and Mr. L. E. Parsons, who did the collecting, gathered more than ro cwts. of matrix which I received in the Isle of Man; without it, this work would have been far less successful. Mr. C. R. Cuthbert, Metropolitan Police Laboratory, Scotland Yard, Camp Commander at the Married Aliens Internment Camp on the Isle of Man from I94I to I944, made it possible for me to work on the material during our stay on this island. Mr. J. R. Bruce, acting Director of the Marine Biological Station of Liverpool University at Port Erin, offered the facilities the Station could provide; thus the setting of fragments and the finer preparation were done with the help of binocular microscopes. Mr. N. A. Barnicot greatly helped to improve the author's English. The material had been worked out in the Zoological Department of University College, London, under the benevolent guidance of Professor D. M. S. Watson, and the writer is deeply obliged for the patience, kindness and permanent interest Professor Watson has shown to him. Thanks are also due to Dr. C. T. Trechmann, and to my wife, Mrs. Charlotte Kühne, who was present when the site was discovered in August 1939 and who collected many exquisite specimens. Mr. D. E. Woodall did the excellent drawings of most of the specimens under my supervision. Mr. W. N. Edwards, Keeper of Geology at the British Museum (Natural History), and members of his staff, particularly Dr. W. E. Swinton, have assisted me in various ways.

The first part of this monograph was presented in 1949 to the Mathematisch-Naturwissenschaftliche Fakultät der Rheinischen Friedrich Wilhelms Universität at Bonn in candidacy for the degree of Doctor rerum naturalium. 


\section{GEOLOGY}

Numerous workers have shown that Rhaetic and Liassic beaches and islands are today exposed in Somerset and Glamorgan. Immersion-horizons and derived fossils are characteristic features of the Liassic shelf, beach-deposits and fissure fillings of the contemporaneous shorelines and islands. It is in the fissure fillings that one has to look for sediments containing representatives of Rhaetic and Liassic mammalian and pre-mammalian life. For this reason the author studied the fissure filling in the Carboniferous Limestone of the Mendips and of South Glamorgan. In both areas the Carboniferous Limestone is extensively quarried and consequently well exposed. Fissure fillings can be observed in almost every other quarry. The fissure sediments are always more or less conglomeratic and either argillaceous -from which the extraction of the micro-fauna is relatively easy-or calcareous. These fissure fillings are often said to be of Rhaetic age and this is confirmed by superficial investigations, which will almost always produce Rhaetic fishes. But it would be wrong to accept the Rhaetic age of a fissure deposit on the evidence of teeth of Acrodus minimus, Saurichthys acuminatus and Sargodon tomicus unless this is confirmed by the absence of fossils of a younger age. It is only natural to expect these very compact teeth, in perfect preservation, to occur as derived forms in younger deposits. To argue the age of one fissure filling from another in the neighbourhood would be unwise, because fissure fillings ranging in age from the Rhaetic to the Vesulian are to be expected side by side. The only reliable way of establishing the age of a fissure deposit is to determine all its fauna, and especially its invertebrates. Like the Microlestes-locality Holwell, the fissure " Mendip I4," containing the Oligokyphus bone bed, is situated on the south flank of one of the Mendip periclines. Like Holwell, "Mendip I4" contains a faunal assemblage which ranges from the Rhaetic to the jamesoni zone of the Charmouthian. If the terrestrial vertebrates found in the fissures are waterworn, or if the possibility of secondary deposition in the fissure deposit cannot be safely ruled out, their exact age determination is impossible and only a minimal and maximal age of the determinable elements of the marine fauna can be given.

\section{The Locality " Mendip I4"}

The locality "Mendip I4" is the Windsor Hill Quarry, one mile north of Shepton Mallet. This quarry, now disused, stands entirely in the Zaphrentis zone, its northern boundary being actually in the basal shales which lead down into the Old Red Sandstone. In the east and south, the Lower Lias approaches to within a few hundred yards of the quarry; in the west the Carboniferous Limestone continues for about a mile, with only isolated patches of Mesozoic cover. The Carboniferous Limestone has a dip of about $35^{\circ}$ to the south-west; the fissure which contains the bones of Oligokyphus strikes east-west. The fissure is only one foot wide or less and is exposed for about 50 yards. It was fortunate that the outcrop of the fissure was so near to the road that quarrying had to stop, and that the fissure was thus extensively exposed. The fissure deposit is a rather pebbly, ferruginous, sandy limestone, the pebble content increasing to the west. Bones of Oligokyphus have 
been found all over the exposure, but the collecting was done mainly in the western part, where the yield of bones was greatest. The data given below refer to this part of the exposure. The colour of the fissure deposit is a light brown. Stratification is perceptible, the dip of the bedding planes being $10^{\circ}$ to the west. Of the comminuted matrix, ranging from 0.5 to $1.0 \mathrm{~mm}$., 80 per cent. is soluble in cold $\mathrm{HCl}$; the residue consists of some brown clayey material and plenty of rounded quartz grains. Sixty per cent. of the pebbles over $5 \mathrm{~mm}$. are Carboniferous chert, 30 per cent. Carboniferous Limestone and unidentified limestone containing no fossils and possibly of Triassic age, Io per cent. are silica pebbles. Chert and silica pebbles have a high polish and the latter are often deeply cavernous, indicating that minerals which before the formation of the fissure deposit were part of the pebble have been leached out. Rare angular and relatively large pieces of Carboniferous Limestone were probably derived from the walls of the fissure or from the immediate neighbourhood. Rare too, but conspicuous by their purple colour, are pebbles and derived fossils of phosphatized Lias, the largest fragments measuring not more than $4 \mathrm{~cm}$. The only other macroscopic components of the fissure sediment are the bones of Oligokyphus with a maximum length of $6 \mathrm{~cm}$. ; in a few very rich slabs of matrix as many as 200 specimens per cubic foot were encountered. Here and there in the fissure deposit are patches dark grey in colour, due to finely disseminated pyrites. There can be no doubt that before weathering the whole of the fissure deposit was pyritous. The tractability of the matrix, which allows even the smallest skeletal elements to be freed, is due to the weathering of pyrites. Calcite-cement was transformed into soluble selenite and this was subsequently leached out, leaving the originally compact and hard matrix loose and locally friable. Evidence for slumping and settling of the fissure deposit has been found only occasionally; for instance a large lower incisor and a rib have been found which had been fractured after their sedimentation. The fragments met at an obtuse angle, the fracture planes lying opposite each other.

\section{Stratigraphy}

The conditions under which the deposition of the bones of Oligokyphus took place at "Mendip I4" are described in detail in the following chapter on biostratonomy; unequivocal evidence is there produced that the bones of Oligokyphus were deposited in the fissure already macerated, and in some cases superficially weathered, but otherwise still fresh. It is certain that the bones are in their primary place of deposition.

The time of deposition of a sediment containing a mixed fauna is determined by the youngest components of this fauna. As will be seen, only a small part of the marine fauna of "Mendip I4" is of value in determining this time; the greater part of the fauna is either derived or unsuitable as a time marker.

The fishes of the "Mendip I4" fauna have been determined by Dr. E. I. White, the ammonites by Dr. L. F. Spath, the belemnites by Mr. L. Bairstow, other molluscs by Dr. L. R. Cox, and the brachiopods by Dr. H. M. Muir-Wood.

\section{VERTEBRATA}

FAUNAL LIST

Oligokyphus major n. sp. (see p. 98). The genus is also known in the Upper Rhaetic.

Oligokyphus minor n. sp. (see p. 98). 
Acrodus minimus. Ten teeth. Middle Trias-Rhaetic.

Acrodus anningiae. Two teeth. Lower Lias.

Hybodus minor. Few teeth. Rhaetic-Lower Lias.

Saurichthys acuminatus. Five teeth. Trias-Rhaetic.

A Chimaeroid ; more than 400 dermal tubercles.

\section{AMMONOIDEA}

Armioceras sp. | both specimens are imprints on the attached valve of oysters. Lower Promicroceras sp.\} Lias.

There are numerous indeterminable casts of interseptal spaces of ammonites preserved in phosphate and derived.

\section{BELEMNOIDEA}

The numerous belemnites which are found especially in the western part of the fissure deposit, where it is more conglomeratic, are badly preserved. Moreover, the British belemnites from the Lower and Middle Lias are greatly in need of revision. The belemnites from "Mendip I4" can be compared with "Belemnites acutus," confined to the planorbis and bucklandi zones and with Pseudohastites sp. which occurs in the Charmouthian. It is certain that most of the belemnites, though indeterminable specifically, actually belong to several species of the Pseudohastites group; this group is definitely Charmouthian and, furthermore, a rich belemnite fauna, as realized at "Mendip I4," is not found before the Charmouthian in England. There is one phragmocone well preserved in phosphate and derived.

\section{GASTROPODA}

Indeterminate small gastropods are frequently found in the pebbles of derived Liassic phosphate.

\section{LAMELLIBRANCHIATA}

In the derived Liassic phosphate, three indeterminate internal casts of Homomyaria have been found. The underived lamellibranchs are all Anisomyaria. They are badly preserved and either allow no specific determination or are unsuitable as zonal indicators:

Oxytoma inaequivalve; Pecten; Ostrea; Gryphaea; ? Pseudolimea; Meleagrinella; Inoceramus.

\section{BRACHIOPODA}

The brachiopod fauna is varied and its preservation is such as to allow specific determination. The report by Dr. H. M. Muir-Wood is as follows :

" The Brachiopoda consist of 47 fragmentary detached valves, one immature specimen with shell entire and sixteen derived specimens; some of the derived specimens, though imperfectly preserved, have two valves attached. The deposit appears to be remanié, and most of the specimens are much dwarfed. 


\section{'Derived’ Specimens}

" The specimens labelled 'derived' have been identified as :

"(I) Calcirhynchia? sp. Part of a pedicle valve. Calcirhynchia calcaria S.S. Buckman, to which species this fragment may belong, occurs commonly in the Hettangian, angulatum zone, and in the Sinemurian, bucklandi zone.

"(2) Spiriferina tumida (von Buch). Five imperfect specimens, not dwarfed, four of which have the two valves attached.

"Distribution. Hettangian, angulatum zone, and Lias $\alpha$ of Württemberg. In Dorset this species has been found by Dr. W. D. Lang in the angulatum zone of the coast-section.

"(3) Spiriferina walcotti (J. Sowerby). Five imperfect specimens with both valves attached, one of which is nearly normal size, the other four being about half adult size.

"Distribution. Sinemurian, semicostatum zone, gmuendense subzone of the Radstock area, and in a nodule bed in phosphatized condition at the base of the turneri clay.

" (4) Zeilleria sarthacensis (d'Orbigny). An internal cast of the posterior half of both valves preserved in limonite, and part of a pedicle valve, both normal size.

"Distribution. Z. sarthacensis is very common in the Hettangian, angulatum zone, but does occur at the base of bucklandi (Sinemurian).

"(5) Squamirhynchia fronto (Quenst.). One specimen normal size with both valves attached.

"Distribution. Lias $\gamma$ of Württemberg; raricostatum (Watch Ammonite Stone Bed 99), and jamesoni (Bed II4) of the Dorset coast ; armatum-jamesoni zone of Radstock district, Somerset.

“ (6) Semiplicate Rhynchonellid preserved as an imperfect internal cast $5 \mathrm{~mm}$. in length. Both valves are moderately convex and there is no fold or sinus. The general form and ornamentation are rather comparable with that of Scalpellirhynchia scalpellum (Quenst.) from the Lias $\delta$ of Württemberg, and from the Lower Lias, Green Ammonite Beds, davoei zone, and Middle Lias, margaritatus zone, of the coast-section, Dorset.

"Specimens I-4 are from the Hettangian or Lower Sinemurian, while 5 and 6 occur elsewhere in the Upper Sinemurian or the Pliensbachian.

\section{"Specimens Presumed to Be Contemporaneous}

"These specimens have been identified as:

“( (I) Spiriferina walcotti (J. Sowerby). Five detached valves about half normal size.

"Distribution. Semicostatum zone, gmuendense subzone of Somerset, especially Radstock district.

“(2) Piarorhynchia radstockiensis (Dav.). Four fragments, all dwarfed.

"Distribution. As for S. walcotti.

"(3) Piarorhynchia sp. Three fragments.

" Distribution of Piarorhynchia sp. Identified as an undescribed form from Table Ledge, Dorset, Bed 53 of Lang, semicostatum zone, scipionianum subzone of Spath.

" (4) Calcirhynchia calcaria S. S. Buckman. Six fragments, all dwarfed.

"Distribution. Hettangian, top of angulatum zone, and Sinemurian, base of bucklandi zone of Dorset, Somerset, and Glos.

"(5) Rhynchonellid sp. indet. Fragments of two species. 
“(6) Squamirhynchia fronto (Quenst.). One imperfect brachial valve, dwarfed. Identical in ornamentation with specimens from Radstock, and Dorset (Lang Coll.). Also found derived.

"(7) Spiriferina verrucosa (von Buch) var. plicata (Quenst.). One brachial valve, dwarfed.

"Distribution. Armatum zone of Radstock, and Glos; Lias $\gamma$ of Württemberg.

“(8) Zeilleria cf. crithea (d’Orb.). Fifteen fragments.

"A general comparison with the Lias Zeillerias showed that the fragments resemble most closely Z. crithea from the armatum-jamesoni zone of Radstock.

"Specimens I-5 are from the Hettangian or Lower Sinemurian, while specimens 6-8 are from the Upper Sinemurian or the Pliensbachian."

The evidence from the brachiopod fauna is rather equivocal. There are two components of Sinemurian age, one derived and of normal size, the other underived and dwarfed. The third component consists of Charmouthian forms, of which Squamirhynchia fronto occurs underived and dwarfed, and derived, filled with phosphatic rock and of normal size. This evidence, taken in conjunction with that obtained from the belemnites, is sufficient for it to be stated quite definitely that the age of deposition of the fissure filling "Mendip I4" containing the bones of Oligokyphus sp. is Charmouthian (Lias $\gamma$ ).

Oligokyphus triserialis, the type species of Oligokyphus, and Oligokyphus biserialis come from the "Rhaet-Lias" of Württemberg. The material consists of one upper and one lower cheek tooth, both waterworn. Both teeth are deposited in the Geological and Palaeontological Institute of Tübingen University. Oligokyphus has now a recorded biozone from the "Rhaet-Lias" to the Charmouthian. 


\section{BIOSTRATONOMY}

This section is based on observations and records made while preparing the bones of Oligokyphus in the period I94I-I944. During these war years, considerable time was at my disposal and the matrix available was only Io cwt., obtained from Somerset by the Geological Department of the British Museum. Not being pressed for time I was able to give the same attention to specimens which were morphologically valueless as to those of morphologic importance. The restricted amount of matrix made it desirable to extract all the bones in it. The result was: firstly, that even very small bones were found. For instance, I owe to this procedure the six specimens of quadrate. Secondly, that as no material was neglected it became possible to make a quantitative assessment of the fragmentation of the limb bones. Thirdly, the quantitative relations of different skeletal elements as represented in the matrix could be determined.

The vertebrate palaeontologist is often forced to use very fragmentary material and this is rightly considered a nuisance. None the less, I hope to show that the fragmentation and numerical representation of bones may be a characteristic feature of a fauna and that its analysis may yield valuable information.

I have looked in vain through the literature to find papers dealing with fracture incidence and fracture edges of Recent terrestrial animals whose cause of death is beyond dispute. Such work would have made my own conclusions much more reliable. ${ }^{1}$

\section{Summary of the Evidence}

I. The matrix of "Mendip I4" is a marine fissure filling. Natural cavities in bones, for instance nerve channels, as well as those cavities which originated when the marrow cavity was exposed by fracture, are filled with the same matrix which surrounds the bones.

2. No two associated bones have been found-this was still true in I948 when the total number of bones prepared was at least twice as large as in I944. This excludes of course fragments of the skull and one specimen of sacral vertebrae, where the association is by co-ossification or by suture.

3. Bones found in small pieces of matrix may differ considerably in colour. The colour of bones and teeth ranges from light brown to almost black. Light bones are well preserved, their surface is smooth, their structure is compact; dark bones are less well preserved, their surface is rough and exfoliated, their structure is porous. The colour differences of bone are independent of the state of the matrix.

4. Very slight water wear is detectable on fracture edges, similar in degree on all specimens. Articular ends of bones, when preserved, are never damaged by water wear. Wear facets on incisors and cheek teeth are almost always sharp and perfectly preserved.

5. It is very remarkable that among the total number of specimens collected up to I95I, not one bone or fragment of bone has been found which could be assigned to a terrestrial vertebrate other than Oligokyphus.

\footnotetext{
1 The author of a paper on the fracture incidence and the fracture edges of the bones of a herd of Rangifer, chased by wolves over precipices in Lapland, would endear himself to all palaeontologists working on mammals.
} 
6. The majority of the small bones of pes and manus are complete, but with increasing length and complexity, complete bones become rare. For instance: among IIO registered specimens of limb bones, only two tibiae, one radius and one ulna have been found complete. Of vertebrae 50 per cent. have been found complete, though the dorsal spine was missing in almost all specimens; of metapodials and phalanges 80 per cent. have been found complete.

7. Carnivore tooth marks can be observed on the bones of Oligokyphus.

8. The numerical representation of different skeletal elements in the matrix does not correspond to their representation as realized in whole individuals; on the contrary, a considerable discrepancy exists. The maximum number of whole individuals as represented by upper cheek teeth contrasts with one tenth of this number represented by carpalia and tarsalia.

These facts make it possible to deduce the history of the bones of Oligokyphus from the moment of death until their entombment in the matrix. Besides this a little can be said about the geographical and climatic environment of Oligokyphus, about the organization of Oligokyphus itself and about the unknown carnivore which preyed on it.

There is nothing to suggest a marine or aquatic habitat for Oligokyphus. All the evidence given in the osteological part of this monograph is, without reservation, for a terrestrial habitat. It is, therefore, legitimate to assert that Oligokyphus met its death on dry land and that flowing water brought the skeletal remains into the marine matrix where the bones are found.

\section{Interpretation of the Evidence}

(I) The presence of the same matrix inside the bones as well as surrounding them is an indication that the bones are found in their primary place of sedimentation. Evidence that the bones of Oligokyphus were not derived is also provided under (6) and (8).

(2) The total absence of associated bones in the locality means that maceration of the skeletal elements and fragments was complete before sedimentation took place. If this were not so one would expect to find associated skeletal elements as least as rarities. Whatever the source of supply of the bones of Oligokyphus on dry land may have been, a continuous transportation of bones to the fissure "Mendip I4" is out of the question because of the absence of associated elements. Continuous transportation would have brought bones from animals so recently dead that their bones had not been macerated.

(3) Colour differences between bones in the same small piece of matrix are independent of the state of the matrix, and dark colour is correlated with exfoliation, light colour with smooth and intact bone surface. Exfoliation is a consequence of sub-aerial weathering; it cannot be brought about while a bone is immersed. It is therefore legitimate to say that colour variations in different skeletal specimens of Oligokyphus are a consequence of different lengths of exposure to weathering, due either to the bones having accumulated slowly on dry land and later having been swept together into the sea, or to the varying period that elapsed before specimens became waterborne. If the first alternative held, one would have expected a similar percentage of all kinds of specimens to have a certain degree of lightness. Actually this is not the case, and observations support the second alternative. Using as a standard a certain lightness of the teeth which have a colour-range similar to that of the bones, it was found that isolated upper cheek teeth had a light : dark ratio of I : II ; for the same 
teeth in situ this ratio was I : 7.5; for isolated upper cheek teeth of very small size-possibly the teeth of quite young animals - the same ratio was I : 3.5. Specific gravity and form determine the resistance of a body to flowing water. Hence it required less water-power to transport the minute cheek teeth from land to sea than the cheek teeth of normal size. It required less water-power to transport from land to sea maxillae with teeth than isolated upper cheek teeth of the same size. It follows that weathering before transport took place over longer periods in some specimens than in others. This conclusion can be corroborated by another observation : 60 specimens of dentaries have been distributed according to their colour into three groups of 20 , light, medium and dark, and the empty alveoli and cheek teeth in situ have been counted. The result is this:

$\begin{array}{lcc} & \text { Empty alveoli } & \text { Teeth in situ } \\ \text { Light group } & 30 & 26 \\ \text { Medium group } & \text { I7 } & 38 \\ \text { Dark group } & \text { I3 } & 40\end{array}$

the respective ratios are : I.I5: I ; $0.44:$ I ; $0.32:$ I.

(4) All the fracture edges are less sharp than those produced while preparing the bones. Really rounded edges have never been observed, nor have bones been found in all stages of water wear; the homogeneity of the whole material is impressive in this respect. The conclusions from this evidence are obvious. The whole skeletal material found at "Mendip I4 " had an extremely short journey from dry land to the fissure in the sea-floor. The material with skeletal remains of Oligokyphus did not come from the whole catchment area of even a small stream but it must have originated from one small site. Two conditions served to prevent the bones from suffering breaker action. Firstly, the bones had to be fully macerated and air could easily escape through the nutritive foramina when the bone became immersed-this prevented the bones from floating on the surface. Secondly, the bones must have been carried right out into the sea through a small river mouth. Prof. H. Gerth kindly pointed out to me that any one bone found in the fissure would escape the effects of surf and breakers if transported to the fissure during low tide, the fissure being in the intertidal zone or very near to it.

(5) The complete absence in the fissure deposit of any terrestrial animal but Oligokyphus is striking. About I5 tons of fissure deposit have been handled; not only has the yielding matrix been comminuted to fragments of less than one-eighth of a cubic centimetre, making possible the extraction of minute and relatively rare bones of Oligokyphus, but the fine sand dust of many hundredweights of matrix was conscientiously sifted, washed, and handpicked. The result was always the same: smaller and still smaller teeth of Oligokyphus were found, and numerous dermal ossifications of a Chimaeroid were met with regularly, but no indication even of Diapsids, let alone mammal-like reptiles or mammals, was encountered. Masses of matrix yielding no bones of Oligokyphus were also comminuted. In fact nothing was left untried to get hold of even a single tooth of a Triconodont, and the result was negative. At "Mendip I4," where ideal conditions for the preservation of Liassic Mammalia appear to have been realized, their total absence is disappointing.

There can be little doubt on geological grounds that the habitat of Oligokyphus was an island of less than 20 square miles. An island habitat tends to favour a few genera which 
are abundant and eliminates the rest of a fauna which might still be found on the mainland. The population of Oligokyphus must have been dense to make possible a bone bed of the abundance of "Mendip I4".

Tooth marks of animals not congeneric with Oligokyphus have been found on its bones, supplying definite evidence of at least one other genus of terrestrial animal in the same habitat. Its absence can be accounted for if we assume between it and Oligokyphus a predator-prey relation such as exists, for instance, between weasel and lemming or fox and rabbit. If the skeletal remains of Oligokyphus are the "left-overs" of a carnivore it is unlikely that bones of this carnivore would be found with those of its prey. That it preyed only on Oligokyphus is probable under the assumption of a restricted insular fauna. That the predator has not even left one tooth in or together with the bones of Oligokyphus makes it likely that the predator did not possess a dentition with rapid tooth replacement. Crocodiles for instance invariably leave great numbers of their teeth with the bones of their prey.

(6) The evidence provided by the fractures of the bones of Oligokyphus will be dealt with under three headings: (a) the conditions under which fracturing occurred; $(b)$ the fracture edges; $(c)$ the location of the fractures.

(a) The great frequency of fractures in limb bones and their relative rarity in metapodials and phalanges make it extremely unlikely that the bones have been fractured in a fossil or sub-fossil condition, for had that been the case, the small and fragile bones of pes and manus would be at least as fragmentary as the limb bones, if preserved at all. This corroborates the conclusion that the bones were not derived.

The conclusions under (2) make it clear that all waterborne bones or bone fragments were already macerated ; that means their specific gravity was greater than water and they sank. Conclusions under (4) give the observed water wear on bones. It must be admitted that bones exclusively fractured by water have not been studied. Fracturing of bones can be expected in rapidly flowing streams, especially with bones heavier than water, as in the present case (since all the bones had been macerated). However, a high fracture incidence would be expected under such conditions and not the very selective fracturing realized at "Mendip I4". This seems to rule out fracturing of the bones while waterborne. Fracturing of bones on land can be accomplished in only a few ways: through falls or similar effects of gravity, and predator action. In both cases no comparable Recent material exists. The only material with which to compare the bones from "Mendip I4" comes from Pikermi ; here the observations though general and not quantitative are reliable; the conclusions are however of necessity hypothetical, as they are not backed by evidence from Recent material.

(b) Three characters of the fracture edges of the limb bones of Oligokyphus will be discussed : the relation of the circumference of the bone to the vertical extension (height of the fracture), the straightness or ruggedness of the fracture edge, and the angle between the fracture surface and the bone surface.

Text-fig. I shows drawings of impressions made by rolling five fractured ends of limb bones of Oligokyphus on plasticine. The relation of their circumference to the height of the fracture is : I: $0.25 ; \mathrm{I}: 0.25 ; \mathrm{I}: 0 . \mathrm{I9} ; \mathrm{I}: 0.26 ; \mathrm{I}: 0 . \mathrm{I} 9$. The ruggedness of the fracture edge is well seen in the figure. In the course of the fracture, two or three corners are produced as well as numerous small indentations. A straight course of the fracture at an acute angle to the axis of the bone rarely occurs. 
The angle between the fracture edge and the surface of the bone is variable. If a bone of Oligokyphus is fractured now, the fractured edge is invariably at $90^{\circ}$ to the bone surface ; the same is the case if Recent bones are burned and afterwards fractured. The bones of Oligokyphus must therefore have been fractured when still elastic, and before the organic matter was destroyed.

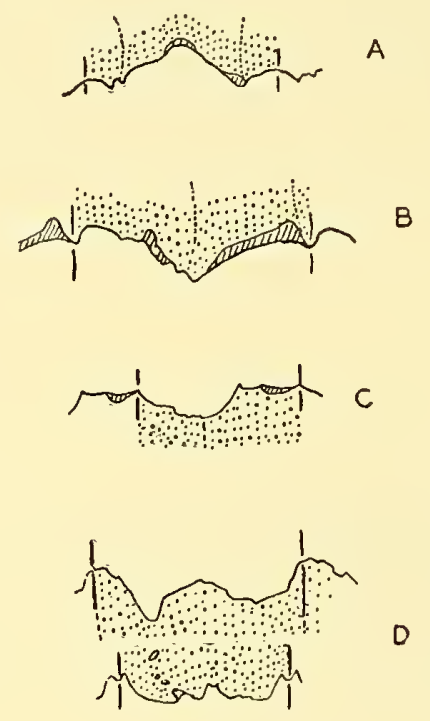

TEXT-FIG. I.-Drawings of impressions of five fractured ends of limb bones of Oligokyphus. The dotted area is bone ; the obliquely hatched area is fracture edge forming obtuse angle with surface of bone, therefore visible externally. One circumference is enclosed between two vertical bars. Dotted line in A and B : Linea aspera and ridge from greater trochanter. Dotted line in C: Deltopectoral crest. A, R.7305, and B, R.7306, femora. C, R.7307, humerus. D, R.7308, tibia.

The limb bones of Hipparion from Pikermi show fracture edges which are very unlike those of Oligokyphus and which have been attributed by Abel (IgI2) to a fall over a cliff edge when the animal was still alive. The ratio of bone circumference to height of fracture is $I$ : 0.65 (average from I8 specimens of tibia). Ruggedness of the fractured edge is not very conspicuous. The angle between the fracture edge and the bone surface is always variable; an angle of $90^{\circ}$ is the exception.

Whatever the ultimate verdict as to the causes of these fractures may be, this evidence shows at least that fractures of a quite different kind are actually found in nature.

(c) In dealing with the location of the fractures of the limb bones of Oligokyphus the procedure has been as follows: all specimens of humerus, femur and tibia, available at a date in 1942 , were arranged side by side so that each fragment occupied its proper place in relation to the whole bone. On the left side of Text-fig. 2 are shown vertical lines which represent the length of each specimen. These lines have been arranged as densely as possible. Each bone is divided into seven equal zones, and the lines dividing the zones have been drawn across the lines representing specimens of the respective bone. On the right side of Text-fig. 2 the dotted and white areas together represent the whole number of specimens in each zone. The dotted area represents specimens ending by fracture in each zone. Dividing the whole number of units in each zone by the number of dotted (fractured) units in each 
zone, the frequency is expressed; the values of fracture frequency are connected by a black line.

The left side of Text-fig. 2 shows a characteristic distribution of the available material ; there is a conspicuous absence of the ends of the three bones. Predator attack on bones always begins at the ends and it is probable that their absence in the Oligokyphus material is due to the predator eating the spongious bone ends, or comminuting them into fragments which are not to be found in the matrix. The spongious ends of bones, once broken off

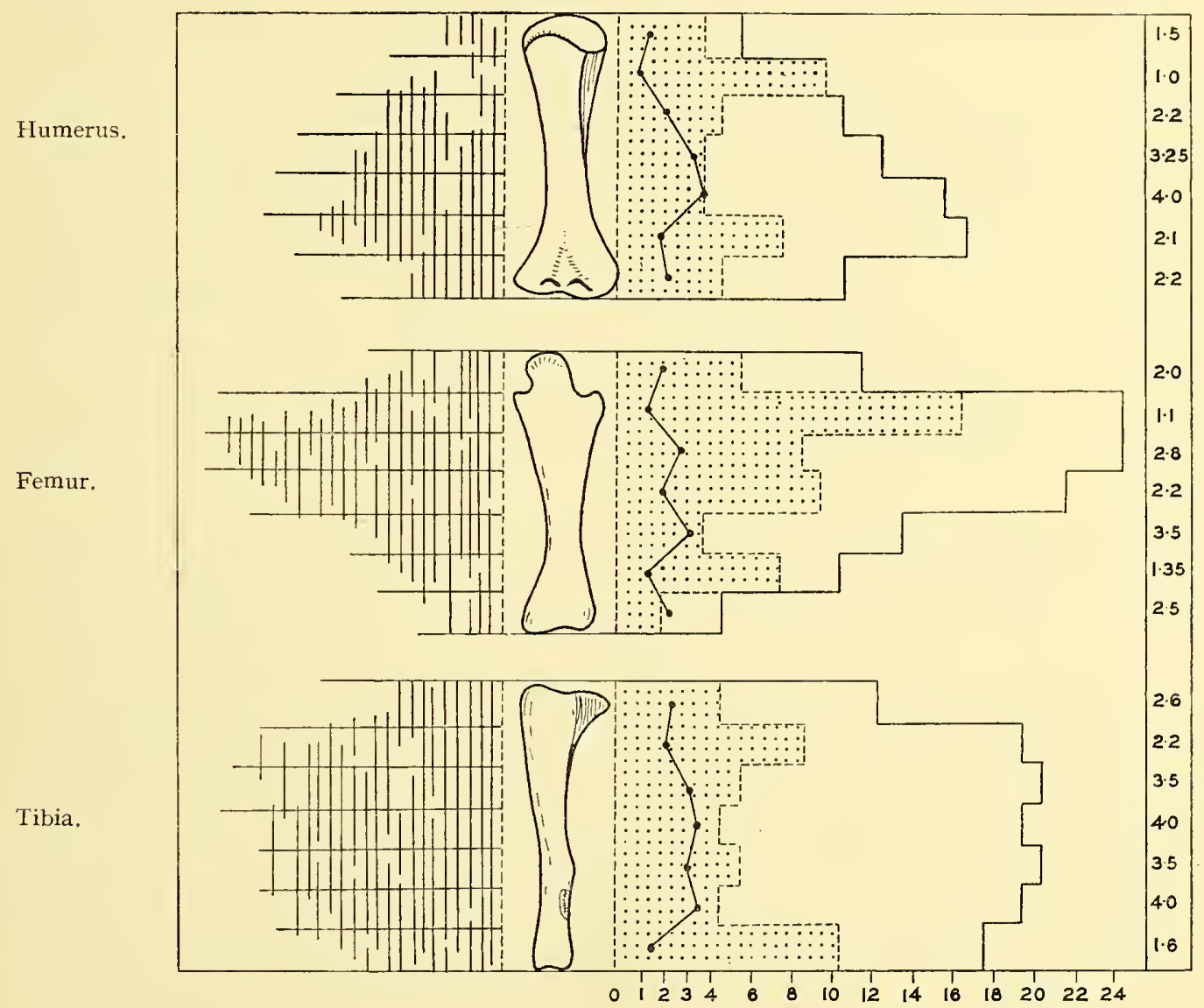

TEXT-FIG. 2.- Location of fractures and fracture frequency in limb bones of Oligokyphus. From left to right: Material divided into seven zones. Outline of limb bones. Dotted area : specimens ending with fracture in zone. The values for fracture frequency are connected by a solid line inside the dotted area. White area : specimens passing through a zone. On the right-hand margin: values for fracture frequency.

the shaft, have however a much smaller specific gravity and offer much less resistance to flowing water than the solid shafts. As will be seen under (8), this resistance plays an essential part in the numerical distribution of any kind of specimen and is the cause of the paucity of small and light skeletal elements in the fissure deposit. It is therefore clear that the absence of ends of limb bones may be due to predator action and to differential fluvial dispersal.

The striking preponderance of the material of tibiae seems to be entirely due to predator action. This bone is the only one of the distal limb bones comparable in strength and 
compactness with humerus and femur. The tibia is so much better preserved because the distal extremity is much less muscular than the proximal and therefore less attractive to the predator, which naturally concentrates its efforts on the extremities, the shoulder and the pelvic region. The rarity of fractures in the bones of pes and manus, compared with the frequency of fractures encountered in other extremity bones, has a similar explanation; pes and manus do not provide food for a predator and are therefore left untouched.

Most important, and a direct expression of the activity of the predator, are the values of fracture frequency for the seven zones, since they are independent of the numerical representation which may be partly due to fluvial dispersal. As will be seen on the left side of Text-fig. 2, the maximum fracturing coincides only on one of six occasions with the minimum representation of the bone (distal end of tibia). The maximum fracture frequency is always associated with the region where the soft spongiosa merges into the dense and hard shaft of the bone. The minimum fracture frequency is found in the proximal extremity bones where their shaft is narrowest. Again the minimum of fracture frequency and the representation maximum do not coincide. The average fracture frequency of all three bones of humerus, femur and tibia is $2 \cdot 3,2 \cdot 17,3.75$; the number of complete bones found up to 1948 for the same elements is: 3 , I and 4 .

The average length of a specimen of humerus, femur and tibia is $0.34,0.36$ and $0.4 \mathrm{I}$ of the whole bone.

(7) The above paragraphs provide circumstantial evidence for predator action on the bones of Oligokyphus. The tooth marks of a carnivore on the bones provide direct evidence. These marks generally occur as pairs of small craters. As their impressions in matrix have been preserved, there can be no doubt that they were inflicted before the sedimentation of the bones. Specimen R.7309 has a round perforation near the fracture edge. In R.7308 (Text-fig. I, D) three tooth marks are arranged in a row, followed on the fracture edge by a large indentation, providing evidence that the tooth-marks and the fracturing of the bone have been caused by the same action. The tooth-marks of rodents are frequently found on bones of Tertiary mammals; but it is obvious that such tooth-marks are not associated with the death of the animal providing the bones. In the case of Oligokyphus this is not so.

(8) When dealing with the numerical representation of different skeletal elements the absolute number of specimens of any kind of bone is not significant. Such a number becomes significant however if divided by the number of times this element is present in a whole individual and compared with numbers similarly obtained of other skeletal elements. The numerical representation of certain elements in an individual of Oligokyphus is not assessable with certainty. The reasons why a certain numerator has been employed when reducing the absolute numbers are given below. The observed number of upper cheek teeth in the maxillary is 6 or 7 . The number of lower cheek teeth is variable from 4 to 6 in small specimens and 7 in large specimens; the numerator chosen is 6 . The numerator for limb bones is obvious ; however, it was necessary to reduce the absolute number of specimens because of the intense fragmentation of these bones. The reduced number is the maximum number of specimens of a bone passing a zonal line in Text-fig. 2. The number of precaudal vertebrae has been determined as 30 , slightly less than in Cynognathus. The numerator for metapodials and terminal phalanges is 20 , assuming a pentadactyle pes and manus. The phalangeal formula has been determined as $2,3,3,3,3$. Oligokyphus is given a mammalian 
phalangeal formula because this formula was well under way in early Cynodonts and it is therefore most probable that the Liassic Oligokyphus already had a mammalian pes and manus. Moreover, if one or several digits had possessed more than three phalanges, the second or the third phalange would be expected to have the same breadth but only half the length or less of the first phalange. Such bones have not been found; if present, however, they would have been found together with many other skeletal elements of similar size. The numerator for phalanges (minus the terminal phalange) is therefore 36 . The numerator for carpalia and tarsalia has been put down as 32 ; this number is reasonable, but will not be argued further.

\begin{tabular}{|c|c|c|c|c|}
\hline & $\begin{array}{l}\text { Total number } \\
\text { of specimens. }\end{array}$ & $\begin{array}{l}\text { Reduced } \\
\text { numbers for } \\
\text { limb bones. }\end{array}$ & Numerator. & Quotient. \\
\hline $\begin{array}{l}\text { Upper cheek teeth, } \\
\text { situ. }\end{array}$ & $2 I O$ & - & I4 & I5 \\
\hline Femur & 33 & 23 & 2 & II $\cdot 5$ \\
\hline Lower cheek teeth & I $2 \mathrm{I}$ & - & I2 & $I O \cdot I$ \\
\hline Tibia & 32 & I9 & 2 & $9 \cdot 5$ \\
\hline Humerus & 26 & I6 & 2 & $8 \cdot 0$ \\
\hline Trunk vertebrae & II3 & - & 30 & $3 \cdot 76$ \\
\hline Metapodials & 75 & - & 20 & $3 \cdot 75$ \\
\hline Phalanges & IOg & - & 36 & $3 \cdot 0$ \\
\hline Terminal phalanges & 49 & - & 20 & $2 \cdot 4$ \\
\hline Carpalia and Tarsalia & 48 & - & 32 & $I \cdot 5$ \\
\hline
\end{tabular}

The quotient, column 4 in the table, gives the number of individuals which can be fitted with the available material. As can be seen, the largest number of individuals-I5-can be fitted with upper cheek teeth; the smallest number (carpalia and tarsalia) is one-tenth of the maximum. The rarest specimens are small and light, the most frequent specimens are heavy or large. That, in the latter group, whole bones are of the greatest rarity can only be explained by the almost complete fragmentation of large bones before being waterborne. That the current which transported the skeletal elements of Oligokyphus carried all of them and did not act selectively, is borne out by the preponderance of the heaviest elements in the fissure deposit. As the current was capable of transporting the heaviest elements to the fissure, it could certainly do the same with the others. The mode of motion along the sea floor was, however, different for each specimen, the heavy ones coming to rest much more often than the light ones. As the fissure was not wider than one foot, the observed distribution of light and heavy specimens in it would be realized if the fissure extended at right angles to the direction of the current carrying the specimens. The heavy specimens taking small steps along the sea floor would fall invariably into the fissure, whereas the light specimens taking long steps had a good chance to be waterborne while traversing the fissure.

\section{Summary of Deductions}

On a small island in the Mendip Archipelago, a restricted but very numerous terrestrial vertebrate tauna flourished, Oligokyphus being the only herbivorous component, an unknown 
mammal or mammal-like reptile being its predator. At a restricted spot only a few hundred metres from the coast, most likely a spring to which Oligokyphus flocked, the killing and eating of Oligokyphus took place. Fracturing of the bones by the predator was accidental and confined to those regions of the body most nourishing; consequently the bones of pes and manus were left unharmed. The spring was visited only during the dry season, the climate being moist enough to allow complete maceration while it lasted. With the onset of a wet season Oligokyphus no longer used the spring; the area around it, covered by bone, was swept by sheet floods and the skeletal debris became waterborne in a brook. This sweeping was not, however, in every year sufficient to carry all the skeletal debris away, heavy elements, and especially isolated teeth, might well have been exposed to weathering for a year or two until an extremely heavy downpour made them waterborne. Once in the brook, the skeletal elements were carried along its bed right into the sea. Into the open fissure in the sea floor there would fall mainly heavy skeletal elements, the majority of the small and light elements, as well as small debris of bone, traversed the fissure and passed out to sea to be dispersed by currents. 


\section{THE SKULL OF OLIGOKYPHUS}

About 300 specimens of skull bones and 50 specimens of lower jaws are catalogued. This number alone would seem to ensure that even the most intricate structures of the skull of Oligokyphus can be elucidated with ease. But this is far from the case. The skull-about $90 \mathrm{~mm}$. long-has been fractured to such a degree that the longest specimen preserved measures only $35 \mathrm{~mm}$. Very few elements have been found in sutural connection, and sutures and natural margins of bone, which are thin and prone to break away when subjected to fracturing, have gone in many cases. Very thin and flat bones are not preserved at all in this numerically very large amount of material, but there are 35 specimens of maxillaries, 24 premaxillaries, 20 frontals, and 20 jugals.

The great bulk of the material consists of fragments, which are generally useless from the morphological point of view and for reconstruction. The sole justification for having prepared them and made them available is that as a result of this work some rare and better preserved specimens were discovered. Furthermore, in each skeletal element one finds one or more fracture zones, and with depressing regularity almost any fragment found proves to be broken within these zones. Another factor which increases the liability of fragmentation is the lack of early fusion of the skull bones of Oligokyphus. Looking through the rich dentition material, one does not find many teeth markedly worn. Oligokyphus was killed before it could attain old age. Few sutures are in consequence fused. Fortunately, the fragments as preserved are uncrushed and undistorted and show as much as a Recent bone.

The skull material of Oligokyphus consists of fragmentary specimens from two adult forms and less frequent specimens from juvenile individuals. Material from the larger adult form is considerably better preserved than the specimens from the smaller adult form, due to greater resistance to fracturing. It need only be said that the morphological differences between the two adult forms are not greater than between two species of small Felidae.

It is not intended to discuss the status of the two adult forms now ; they may be different species, but there are good reasons against this view.

PREMAXILLARY

(Plate I, figs. 2, 3 ; Text-fig. 3)

\section{Material :}

$\begin{array}{cr}\text { Regd. } & \text { Length } \\ \text { No. } & \text { (mm. })\end{array}$

R.700I $4 \cdot 2$

Right ; with alveoli of $\mathrm{I}^{1}, \mathrm{I}^{2}$ and $\mathrm{I}^{3}$ cleared of matrix and anterior sagittal suture.

$\begin{array}{ll}\text { R.7002 } & - \\ \text { R.7003 } & 4 \cdot 4 \\ \text { R.7004 } & 4 \cdot 3 \\ \text { R.7005 } & 2 \cdot 7\end{array}$

Left ; outer wall only, good for position of $\mathrm{I}^{2}$ and $\mathrm{I}^{3}$.

Left ; essential for medial sutures and palatal maxillary suture.

Left ; essential for anterior sagittal suture.

Left; good, small specimen, anterior sagittal suture. 


\section{Material:}

$\begin{array}{ccc}\text { Regd. } & \text { Length } & \text { Side } \\ \text { No. } & \text { (mm.) }\end{array}$

R.7006

R.7007

R.7008

R.7009

R.70IO

R.70II

R.7012

R.7013

R.70I4

R.70I5

R.7016

R.70I7

R.70I8

R.7019-

7024

WGK I
Right; lacking external wall, showing alveoli of $\mathrm{I}^{2}$ and $\mathrm{I}^{3}$ well, essential for palatal maxillary suture.

Right; with root of $\mathrm{I}^{3}$ in situ.

Right ; with root of $\mathrm{I}^{3}$ in situ.

Right.

Right; good sagittal suture.

Right.

Left.

Left ; good posterior sagittal suture and palatal maxillary suture.

Left ; probably juvenile specimen.

Left; good for alveolus of $\mathrm{I}^{1}$.

Right.

Right.

Right.

The total of 24 premaxillaries is a record among the smaller skull bones of Oligokyphus. To make as many specimens as possible eligible for measurement, the length has been taken from the wall of the foramen incisivum to the external side of the bone, at right angles to the longitudinal axis of the skull, across the lamina which separates the alveoli of $\mathrm{I}^{2}$ and $\mathrm{I}^{3}$.

\section{Orientation}

The basis for the orientation of the separate elements of the skull is the assumption that the upper row of cheek teeth is horizontal. The posterior palatal suture of the premaxillary joins the anterior suture of the maxillary. The anterior palatal surface of the maxillary is bent downwards and the posterior palatal surface of the premaxillary continues its direction. Text-fig. 3 shows this orientation.

\section{Palatal viere}

The palatal surface of the bone is not flat, its anterior part in front of the alveolus of $\mathrm{I}^{3}$ turning upward at an angle of about $120^{\circ}$ to the posterior part of the surface, which directly continues the plane of the anterior part of the palatal surface of the maxillary. The anterior palatal surface is rough and the edge between $\mathrm{I}^{1}$ and $\mathrm{I}^{2}$ is rounded. The posterior palatal surface is smooth, its lateral margin sharp, being covered by the distal end of the maxillary. The heart-shaped foramen incisivum is entirely surrounded by the premaxillaries; there are no palatine processes. The dots on the left side of Text-fig. 3, a give the position of all the small foramina which have been observed on the palatal surface in all 24 specimens. The position of the more posterior foramina is not constant. In general there are three such foramina present ; they are vascular and belong to canals which anastomose in the bone. 
The small anterior foramen medial to $\mathrm{I}^{2}$ is known in Gomphognathus cf. mastacus (Broili \& Schröder, I935 : I34, fig. I3) and in Thrinaxodon liorhinus (Parrington, I946: I84, fig. 2). In Oligokyphus the position of this foramen is quite constant and so is its presence, for it is found in all specimens which have the relevant area preserved. The course of its canal is clear in Oligokyphus ; it passes vertically through the premaxillary and emerges in a groove on the internal side of the bone (R.7or3). This groove passes horizontally and posteriorly for about $4 \mathrm{~mm}$. and then fades out. The soft structure pertaining to the foramen and its canal was therefore attached to the premaxillary and not to the medial vomerine septum.

A

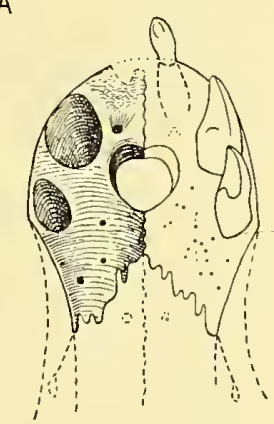

$B$

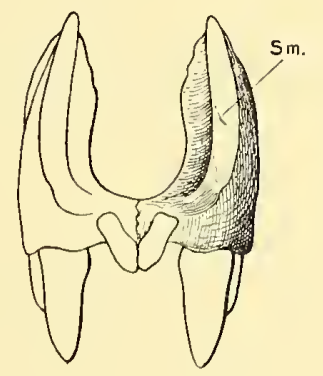

c

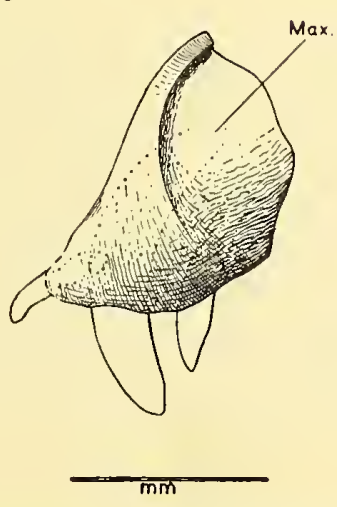

TeXt-FIg. 3.-Premaxillary of Oligokyphus. A, Palatal view. B, Frontal view ; Sm., contact surface for septomaxillary. c, Lateral view ; Max., contact surface for maxillary.

In general the foramen incisivum transmits the ductus nasopalatinus, some vessels and the long spheno-palatine nerve supplying the anterior part of the gum. In this case there is no evidence that the large foramen incisivum served a different purpose, and nothing points to the assumption that the small foramen, in front of the foramen incisivum, has taken over one or more of the functions of this foramen. Most likely therefore the anterior palatine foramen in the premaxillary served only for blood supply.

\section{Lateral View}

The posterior part of the bone is covered by the maxillary, which overlaps it in a gentle and smooth curve. Superiorly the line of contact is followed by a distinct step, indicating that the maxillary is rather massive in this area; this step decreases from above 
downwards and at the lower margin of the bone it is hardly perceptible. The part of the lateral surface of the premaxillary which is covered by the maxillary is characteristically striated; its facial part is slightly pitted. The premaxillary is exposed on the side of the face as a narrow strip between the maxillary and the septomaxillary for the whole of its height.

\section{Frontal View}

The procumbent $\mathrm{I}^{1}$ is conspicuous, as is a slightly concave and narrow surface which begins lateral to and above its alveolus. This surface rises along the anterior margin of the bone to its apex. The upper part of this surface bears the striation which is characteristic of contact areas between two bones, and this whole surface was in contact with the septomaxillary.

The well-preserved specimens R.7002 and R.7004 show that neither a palatine nor an internarial process existed.

By entirely surrounding the foramen incisivum and forming a distinct medial suture behind it the premaxillary of Oligokyphus excludes the vomer and the maxillary from the foramen incisivum. All these characters distinguish the bone from that in Cynodonts.

The measurements reveal a high frequency of large specimens between 3.9 and $4.6 \mathrm{~mm}$. in length. With the exception of R.7oI4 the six smaller specimens indicate fully adult animals. There is no specimen which has the sagittal suture co-ossified.

\section{SEPTOMAXILLARY}

There is no septomaxillary available. According to Broom (I9Io) and Simpson (I928) the septomaxillary of Tritylodon is in contact with the nasal, the maxillary and the premaxillary. Its contact with the last has been discussed above. Neither the available maxillaries nor the nasals are well enough preserved to show the sutures with the septomaxillary. The absence of septomaxillaries from the collection is not extraordinary. Small, oblong and thin bones are in general very rare at the locality.

\section{MAXILLARY}

\section{Material}

Regd.

No. Side

R.7025 Left

R.7026 Right

R.7027 Left

R.7028 ,

R.7029

Cheek
teeth
preserved
$\mathrm{I}-5$
$2-7$

$2-5$

I -5

(Plate I, fig. I; Plate 2; Text-fig. 4)

$\begin{array}{cc}\begin{array}{c}\text { Length of } \\ \text { all C.t. } \\ \text { preserved } \\ (\text { mm. })\end{array} & \begin{array}{c}\text { Length of } \\ \text { C.t. } 2-5 \\ (m m .)\end{array} \\ \mathrm{I} 5 \cdot 5 & \mathrm{I} 2 \cdot 6 \\ \mathrm{I} 6 \cdot 2 & \mathrm{I} 2 \cdot 6\end{array}$

Proximal half of infraorbital canal well preserved.

I5.7 Shows palatal premaxillary sutures.

I3. 4 Shows sagittal and premaxillary suture, facial part above anterior exit of inferior orbital canal.

I5. 8 With lacrimal, jugal and palatine. 


\section{Material :}

\begin{tabular}{|c|c|c|c|c|c|}
\hline $\begin{array}{l}\text { Regd. } \\
\text { No. }\end{array}$ & Side & $\begin{array}{c}\text { Cheek } \\
\text { teeth } \\
\text { preserved }\end{array}$ & $\begin{array}{l}\text { Length of } \\
\text { all C.t. } \\
\text { preserved } \\
(\mathrm{mm} .)\end{array}$ & $\begin{array}{c}\text { Length of } \\
\text { C.t. } 2-5 \\
(\text { mm. })\end{array}$ & \\
\hline R.7030 & Left & $\mathrm{I}-5$ & $19 \cdot 6$ & $16 \cdot 2$ & At anterior end lacrimal suture. \\
\hline R.703I & ,, & $2-7$ & $20 \cdot 7$ & $\mathrm{I}_{5} \cdot 6$ & - \\
\hline R.7032 & ," & $2-6$ & $19 \cdot 6$ & $16 \cdot 3$ & - \\
\hline R.7033 & Right & $2-5$ & - & $15 \cdot 9$ & - \\
\hline R.7034 & ," & $2-5$ & - & I $4 \cdot 9$ & - \\
\hline R.7035 & Left & $2-5$ & - & $\mathrm{I} 4 \cdot 3$ & $\begin{array}{l}\text { A spout for palatine nerve medially } \\
\text { of cheek tooth } 5 \text {. }\end{array}$ \\
\hline R.7036 & Right & $3-6$ & $13 \cdot 2$ & - & - \\
\hline R.7037 & - & $2-5$ & - & $\mathrm{I} 7 \cdot 5$ & - \\
\hline R.7038 & - & $2-7$ & - & $13 \cdot 2$ & - \\
\hline R.7039 & Left & $2-5$ & - & $13 \cdot 3$ & - \\
\hline R.7040 & , & 4 & I3 $\cdot 0$ & - & - \\
\hline R.704I & & $\mathrm{I}-5$ & $\mathrm{I} 4 \cdot 4$ & II $\cdot 8$ & - \\
\hline R.7042 & Right ; & without teeth. & & & \\
\hline R.7043 & & ," & & & \\
\hline R.7044 & Left & , & & & \\
\hline R.7045 & . & $2-4$ & $13 \cdot 6$ & - & Anterior part of infraorbital canal. \\
\hline R.7046 & Right & $3-5$ & II $\cdot 8$ & - & - \\
\hline R.7047 & Left & $4-6 ?$ & $\mathrm{I} 2 \cdot \mathrm{O}$ & - & - \\
\hline R.7048 & Right & 3 & $8 \cdot 3$ & - & May be juvenile specimen. \\
\hline R.7049 & Left & 3 & $8 \cdot 3$ & - & - \\
\hline R.7050 & ," & 3 & $8 \cdot 3$ & - & - \\
\hline R.705I & ," & $3-5$ & $13 \cdot 2$ & - & - \\
\hline R. 7052 & Right & $2-5$ & - & $16 \cdot 6$ & - \\
\hline
\end{tabular}

Fragment of anterior end with facial premaxillary suture, palatal premaxillary suture and sagittal suture.

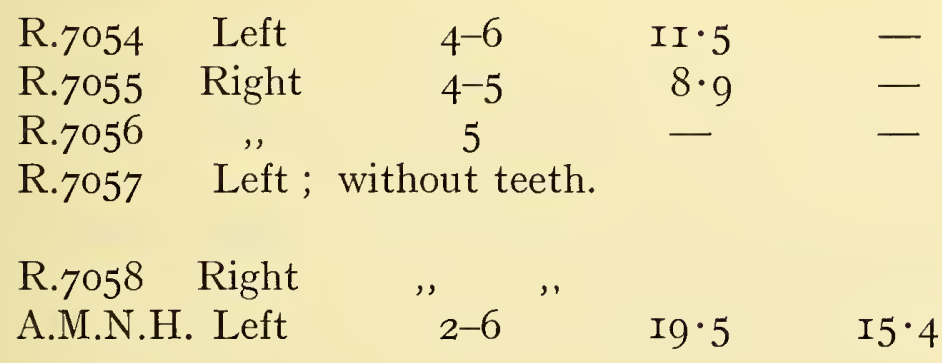

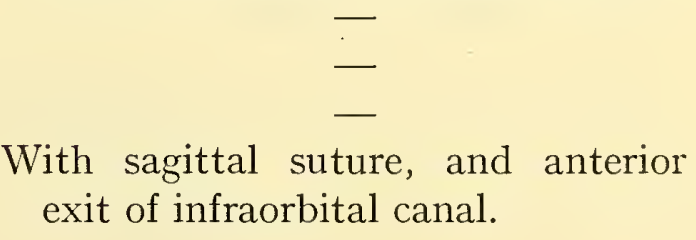

exit of infraorbital canal.

Of the thirty-five fragments of maxillary, twelve have been used in the drawings of the completed bone. The regions still missing are, as might be expected, thin laminae and pointed parts of the bone. But taking into account the evidence from the premaxillary, where the overlapping areas end in an obtuse angle and are therefore preserved, the whole 
anterior area of the maxillary can be reconstructed with certainty. Thus the only area unrepresented even by indirect evidence is the superior-posterior one. The fragment most frequently found is a rod of bone, square in section, ending anteriorly and posteriorly with a fracture and accommodating 3-4 cheek teeth. The total number of upper cheek teeth is seven, of which the last has never been found in use. Fortunately three specimens are less fragmented than usual : R.7027 has been made the basis for the drawings, R.7028 is unique in the preservation of the extremely thin upper facial part, and R.7029 provides the only evidence of the wide lateral horizontal flange on which the jugal rests. Furthermore R.7029 has attached to it parts of the jugal, lacrimal and palatine and is directly comparable with Broili's No. I936 II I of Tritylodon. This specimen is unique and a very useful fragment ; nevertheless its preservation, apart from the association of four bones, is very poor. The consistency of the bone substance is " cheesy", and repeated brushing to produce a clean surface actually deformed the surface-sculpture.

The seven bones in sutural contact with the maxillary are : the maxillary of the opposite side, the premaxillary, the septomaxillary, the nasal, the lacrimal, the jugal and the palatine. Evidence for the sutural contact with the septomaxillary and nasal is derived from Tritylodon and Bienotherium. The bones in sutural contact with the maxillary have remained the same from the earliest Cynodonts to modern mammals, the only changes being due to the loss of the jugal or the lacrimal in a few of the latter. In the Gorgonopsia however a large prefrontal was still in contact with the maxillary.

\section{Sutures}

There is no evidence of any bone being co-ossified with the maxillary. The sagittal suture with the opposite maxillary is shown with the exception of its anterior I mm. The posterior end of this suture is $3.4 \mathrm{~mm}$. wide and has horizontal striae, anteriorly it becomes thinner and the striae change from a horizontal to a postero-inferior antero-superior direction. The palatal premaxillary suture is deeply serrated. Only the second third of the facial contact with the premaxillary is preserved in R.7053. This contact of the maxillary is I mm. thick and abuts against the step provided by the premaxillary between its facial part and the one covered by the maxillary. The posterior end of the premaxillary is received by a pocket in the lateral wall of the maxillary.

\section{Sutures with the Lacrimal}

The suture with the lacrimal begins between the anterior and posterior exit of the suborbital canal and $\mathrm{I} \mathrm{mm}$. above a line connecting them. Externally the maxillary overlaps the lacrimal (R.7028). R.7030 also shows this sutural area and the adjoining floor of the nasal cavity. Conditions in this and the other available specimens make it certain that the exit of the lacrimal duct into the nasal cavity was entirely formed by the lacrimal. The maxillary is not perforated by the lacrimal duct, as it is for instance in Nythosaurus (R.I7I5). The lacrimal is attached to the maxillary by a large sutural area which lies mainly on the flat upper surface of the posterior part of this bone, above the tooth row, but extends to the point where the facial part arises abruptly.

The surface for the attachment of the lacrimal is separated from that for the jugal, which in effect continues it laterally, by the infraorbital canal.

The lacrimal sutural area below the entry of the infraorbital canal almost reaches the 
posterior end of the bone. It leaves a narrow strip of maxillary above the cheek teeth. Anteriorly it is limited by a forward and downward sloping line which ends above cheek tooth 4 and is bounded posteriorly by the sutural area for the jugal. This line cannot be distinguished on the maxillary but can be inferred from the evidence of R.7029 where all three bones are in association and from the jugal R.7087.

A triangular lateral shelf of the maxillary opposite cheek tooth 3 is attached to the anterior end of the jugal by a deep interlock of two horizontal blades from each bone.

A

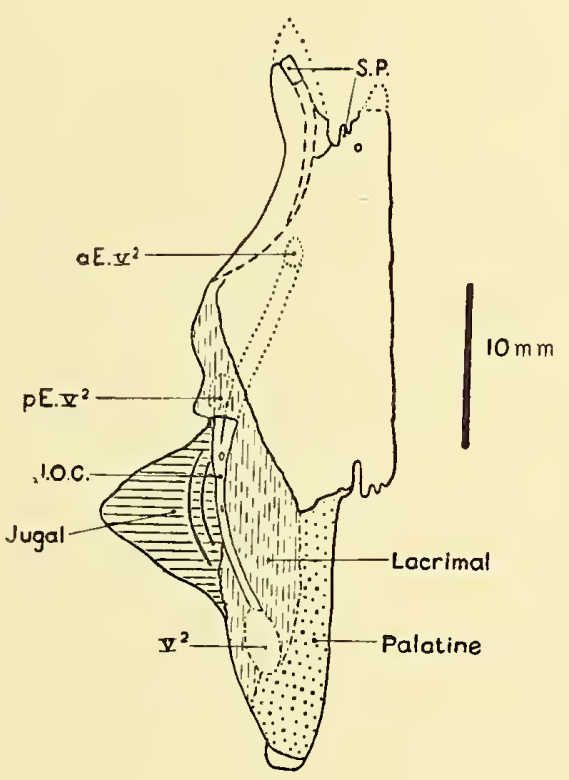

B
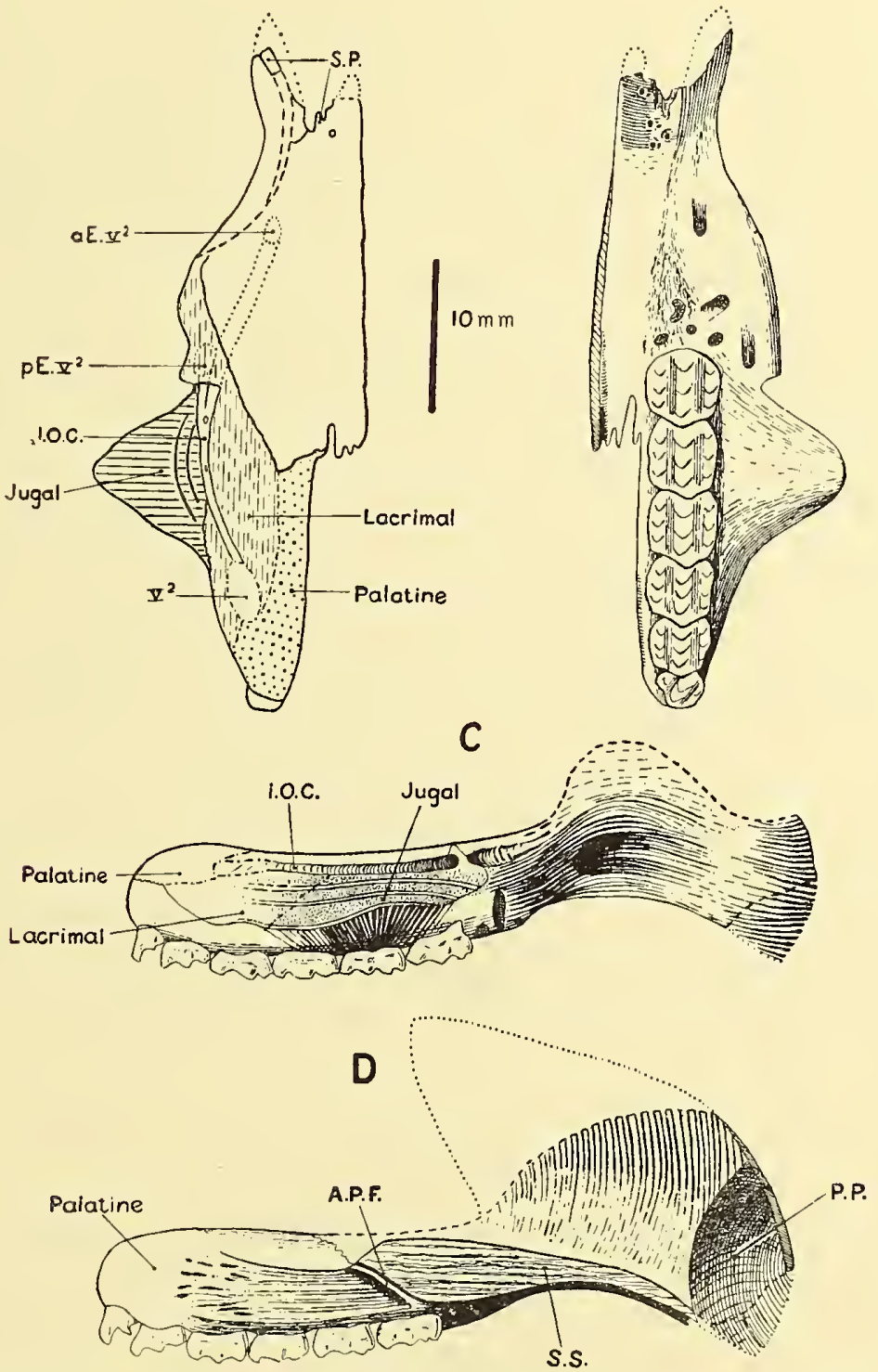

Text-FIg. 4.-Maxillary of Oligokyphus. A, Dorsal view. B, Ventral view. C, Lateral view. $\mathrm{D}$, Medial view. In $\mathrm{A}$ : The sutural areas for jugal, lacrimal and palatine are indicated by different shading. a.E. $V^{2}$, anterior exit of maxillary branch of $\mathrm{N}$. trigeminus; p.E. $V^{2}$, posterior exit of maxillary branch of $\mathrm{N}$. trigeminus ; A.P.F., anterior palatine foramen ; I.O.C., infraorbital canal ; $P . P .$, pocket for premaxilla ; S.P., articulation for premaxillary ; S.S., sagittal sutural surface ; $V^{2}$, entry of maxillary branch of $\mathrm{N}$. trigeminus.

(The teeth in this figure are diagrammatic.) 
The area on the maxillary covered by the palatine is very extensive; it comprises the whole of the internal surface posterior to cheek tooth 2 and the dorsal surface from the end of the bone to the region of cheek tooth 3. The transverse suture between maxillary and palatine, across the palate, is deeply serrated.

\section{Foramina and Canals}

On the palate near the premaxillary suture there are a few vascular foramina. There are two openings for the palatinal nerve: the anterior one passes through the suture between the palatine and the maxillary and leaves a gutter on the latter near the sagittal suture (R.7032, R.7044). The posterior opening for the palatinal nerve is faintly indicated on the maxillary by a shallow groove above cheek tooth 5 (R.7035). This groove flattens out anteriorly and, between cheek teeth 4 and 3, forms a narrow palatal exposure of the maxillary between the cheek teeth and the palatine. A similar condition is found in Diademodon. The infraorbital canal transmits the maxillary branch of the trigeminus. Its entry lies mainly in the lacrimal and palatine (R.7029), but the bottom of this long and oval opening is on the maxillary. This is the case, for instance, in R.7055 and R.7026 ; both specimens also show the medial wall of the canal up to cheek tooth 2. Above this tooth the infraorbital canal is completely enclosed by the maxillary, and sends off its second branch to the face through the maxillary above cheek tooth I (posterior exit of the infraorbital canal). The third branch runs forward inside the maxillary, which is here very shallow, and opens out on the face above the middle of the diastema. There is certainly no other facial exit of the infraorbital canal, though along its course there are several small holes which open into the bone. R.7053 shows conclusively that there are no ossified maxilloturbinals.

The general shape of the maxillary should be clear from Text-figs. 4, A-D and the Plates, and a written description is therefore unnecessary. The following points must, however, be emphasized : the latero-posterior margin of the premaxillary is continued by a sharp edge on the maxillary, separating the palatal from the facial surface. This edge fades out at the height of the anterior exit of the infraorbital canal and is followed posteriorly by a rugose area which ends at cheek tooth I or its alveolus. The sagittal suture and the midrow of cusps on the upper cheek teeth are not parallel and diverge posteriorly to include an angle of $4^{\circ}$. The root of the zygomatic arch extends from cheek teeth $\mathrm{I}$ to 4 but the wide lateral flange on which the jugal rests has its apex opposite cheek tooth 3 .

\section{LACRIMAL}

(Plate 3, fig. 2 ; Text-figs. 4, 5)

There are nine lacrimals available, of which specimens R.7059-66 are isolated and one bone is attached to the maxillary, jugal and palatine in specimen R.7029: of these R.7029, R.7059, R.7064 and R.7066 are essential for the description. Owing to the state of preservation no dimension can be given with accuracy.

The identification of the bone rests on R.7029 and on comparison with Tritylodon and Diademodon; there is no doubt as to its correctness. The bone consists of a basal part attached by suture to the jugal and maxillary, which is penetrated by three systems of canals, and an upper part which forms a large portion of the face and extends inward as a thin lamina to form the anterior wall of the orbit. 


\section{Sutures}

The anterior margin of the facial part, broken but with only a little missing, is well shown in R.7064 and R.7066. Striations on the inner side indicate a sutural contact for the posterior margin of the ascending ramus of the maxillary. The sutures with the body of the maxillary have been discussed above. The suture with the palatine can be observed on R.7029 and R.7064. A thin sheet of palatine covers the posterior part of the lacrimal medial to the entry of the infraorbital canal. The posterior end of the lacrimal in this region is always frayed and broken, but what is preserved shows deep vertical zig-zag sutures (R.7059). The ventral side of the lacrimal, lateral to the infraorbital canal, is attached to the jugal.

\section{Systems of Canals in the Lacrimal}

The infraorbital canal was mentioned when dealing with the maxillary. From its entry, mainly in the lacrimal, to its posterior exit in the maxillary, it runs obliquely through the sutural surfaces of lacrimal and maxillary. At its entry the maxillary forms only the bottom of the infraorbital canal ; the lacrimal, at its antero-medial margin, forms only the roof of it. In the lacrimal the infraorbital canal is bent into a lateral convexity and at the apex of this curve a thin branch is given off. This branch of $V_{2}$ reaches the dorsal surface of the laterally extended base of the bone, after running for two millimetres in it. Three millimetres anteriorly this branch enters into the bone again, to emerge finally at the anterior facial exposure of the lacrimal, immediately above the suture with the jugal. Together with the two exits in the maxillary, we have therefore three facial openings of $\mathrm{V} 2$ in Oligokyphus. The lacrimal branch of $\mathrm{V}_{2}$ is similarly developed in Cynodonts. Simpson (I933 : 293, fig. 5) figured this area in Nythosaurus larvatus (R.I7I5) and held the opinion that a branch of $V_{2}$ emerged from the little foramen below the lacrimal duct. Several specimens of Diademodon in the collection of Prof. D. M. S. Watson show the same foramen which is definitely unconnected with the lacrimal duct.

The lacrimal duct has two openings of equal size, one above the other, in the anteroinferior corner of the orbit. The branches very soon unite. The duct ends on the inner side of the lacrimal in a wide funnel (R.7066). A feature not found in any other mammal-like reptile is a third canal system in the lacrimal. Though close to the infraorbital canal, it is independent of $\mathrm{V} 2$ and lacks any definite relation with a major nerve ; it is probably vascular. From a spot on the lateral inferior margin of the lacrimal and below the anterior rim of the entry of the infraorbital canal, a canal slopes upward and forward and meets the infraorbital canal after a course of $3 \mathrm{~mm}$. Only the lateral wall of this canal exists, and this is divided from the lateral wall of the infraorbital canal by a forward tapering tongue of bone (R.7064, R.7066). The duct leaves the lacrimal together with the lacrimal branch of V2. The presence of small ridges of bone in places where this canal and that for $\mathrm{V}_{2}$ are confluent shows that the structures which lie within them do not unite, but are of a different nature. That the vessel referred to is actually independent of the lacrimal branch of $V_{2}$ is further proved by the fact that their common exit on the horizontal ledge on the lacrimal is about twice as wide as the foramen through which the lacrimal branch of V2 re-enters the bone. In R.7059 all three canal systems have been completely cleared of matrix and the description of their course and relations is based on observation.

From Carboniferous stegocephalians to Recent anthropoids, the lacrimal has a history of 
reduction. A large skull bone acquires a special function-that of harbouring the lacrimal duct - and this function is, in the writer's opinion, a reason why one finds an excessively small lacrimal in numerous mammals. So long as there is a lacrimal duct, the lacrimal can decrease but not disappear. Thus the evolutionary trend of the reduction of the lacrimal is arrested shortly before disappearance. But there is an acknowledged phylogenetic line from Gorgonopsids to Ictidosaurs, in which the lacrimal actually increases in size. The lacrimal of Gorgonopsids is smaller than in early Cynodonts, for instance Thrinaxodon; and in Cynognathus and Diademodon the lacrimal is larger than in Thrinaxodon. The increase in size of the lacrimal reaches its climax in Tritylodontids, which have a face built on the
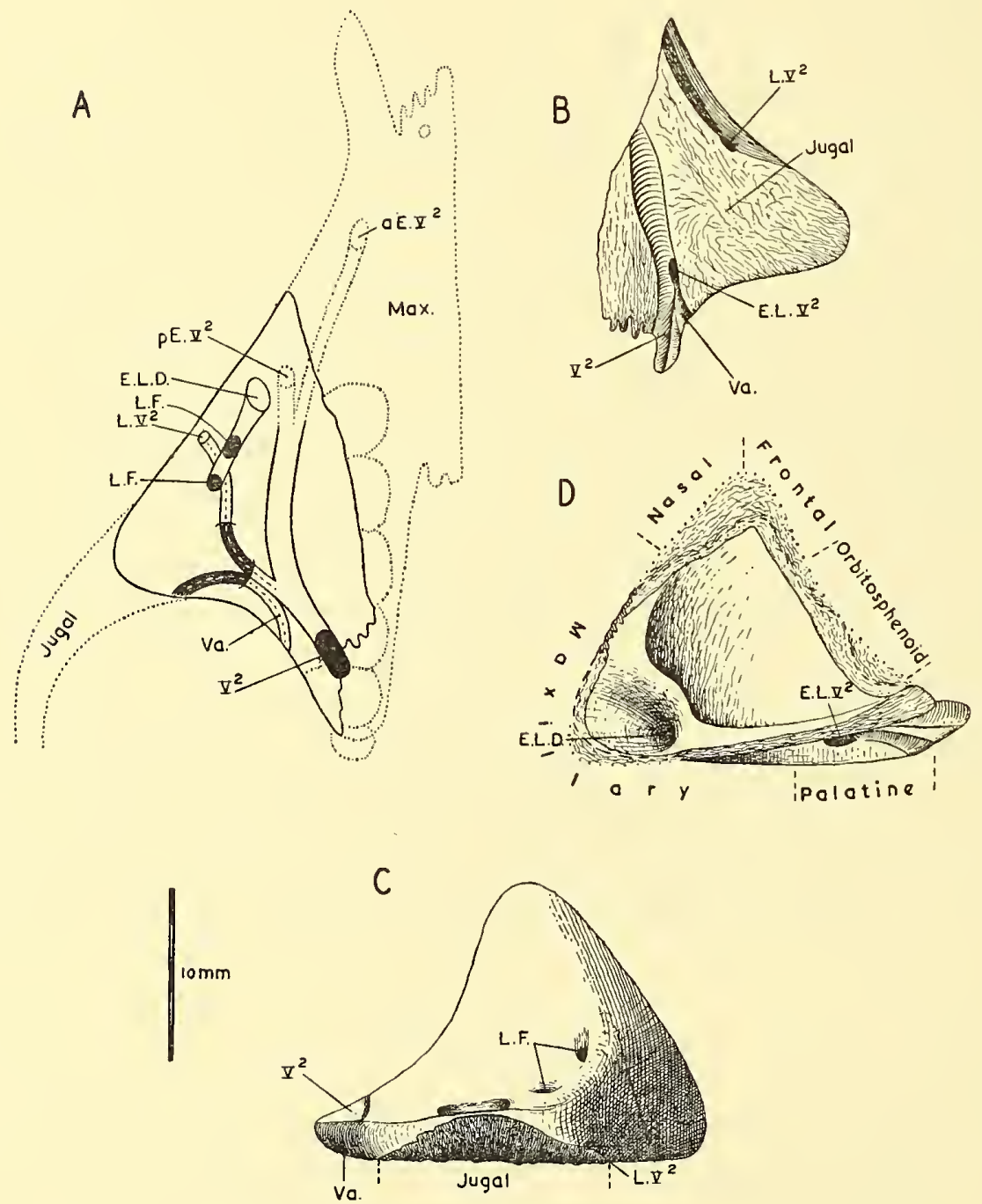

TeXT-FIG. 5.-Lacrimal of Oligokyphus. A, Key to the four systens of canals in the lacrimal. B, Ventral view. C, Lateral view. D, Medial view. a.E. $V^{2}$, anterior exit of $V^{2}$ in maxillary; p.E. $V^{2}$, posterior exit of $V^{2}$ in maxillary ; $L . V^{2}$, exit of lacrimal branch of $V^{2} ; E . L . V^{2}$, entry of lacrimal branch of $V^{2} ; V^{2}$, entry of $V^{2}$ into lacrimal and site of sphenopalatine ganglion ; E.L.D., exit of lacrimal duct ; L.F., lacrimal foramen ; $V a$, vascular canal in lacrimal. In A : The jugal, maxillary and the course of $V^{2}$ in this bone are dotted. In $\mathrm{B}$ and $\mathrm{c}$ : The word "jugal " indicates the sutural surface for this bone on the lacrimal. In D : The words maxillary, orbitosphenoid and palatine indicate the extent of the contact areas of these bones on the lacrimal. 
Cynodont pattern and directly comparable with that of the members of that order. This inversion of an evolutionary trend becomes understandable if one looks for some of the bones which are in contact with the lacrimal. In Gorgonopsids there is a very large prefrontal which during Permian and Triassic times continuously decreased until it is lost in Ictidosaurs. The lacrimal is the bone which gains by the losses of the prefrontal before it likewise decreases and the maxillary gains in turn by its losses. In Oligokyphus, therefore, one would expect a large lacrimal. But the area of the lacrimal is further increased by the nature of its attachment to the jugal. The maxillary contact with this bone is generally vertical, for instance in Cynognathus and Canis, and the lacrimal has no part in the zygomatic arch. In Oligokyphus and the other Tritylodontids the anterior end of the jugal is horizontally flattened. It is inserted between the wide horizontal flanges of the maxillary below and the lacrimal above and thus allows the latter to increase laterally.

JUGAL

Material :

(Plate 3, figs. I, 3 ; Text-fig. 6)

\begin{tabular}{|c|c|c|c|c|c|}
\hline $\begin{array}{l}\text { Regd. } \\
\text { No. }\end{array}$ & Side & $\begin{array}{l}\text { Measurement } \\
(\text { mm.) }\end{array}$ & $\begin{array}{l}\text { Regd } \\
\text { No. }\end{array}$ & Side & $\begin{array}{l}\text { Measuremen } \\
(\text { (mm. })\end{array}$ \\
\hline R. 7067 & Left & $4 \cdot 4$ & R.7080 & Right & $2 \cdot 9$ \\
\hline R.7068 & ,", & - & R.708I & ," & $4 \cdot 4$ \\
\hline R.7069 & ," & - & R.7082 & ," & $2 \cdot 5$ \\
\hline R. 7070 & ," & $5 \cdot 5$ & R.7083 & ," & $4 \cdot 9$ \\
\hline R.707I & ," & $2 \cdot 8$ & R.7084 & Left & - \\
\hline R. 7072 & $"$ & $2 \cdot 8$ & R.7085 & ," & $3 \cdot 9$ \\
\hline R. 7073 &,, & $2 \cdot 6$ & R.7086 & ,, & $2 \cdot 7$ \\
\hline R. 7074 & & $2 \cdot 8$ & R.7087 & ", & $2 \cdot 7$ \\
\hline R.7075 & Right & - & WGK.I & ," & $3 \cdot 0$ \\
\hline R.7076 & , & $5 \cdot 5$ & WGK.2 & Right & $4 \cdot 4$ \\
\hline R. 7077 & ," & $3 \cdot 6$ & WGK.3 & & $3 \cdot 6$ \\
\hline R. 7078 & , & $2 \cdot 6$ & WGK.4 & Left & $3 \cdot 3$ \\
\hline R.7079 & ," & $5 \cdot 5$ & & & \\
\hline
\end{tabular}

Identification of the jugal rests on R.7087, which contains this bone in association with the lacrimal, and on R.7029. The measurement given is the height of the bone immediately behind the insertion area for the lacrimal. The extreme unevenness in distribution of the fragments of the whole bone is noteworthy: there is not one specimen available of the posterior third. The middle part of the bone, with the sutural surface for the squamosal, has been found only four times. All the remaining twenty-one specimens do not reach much beyond the lacrimal-jugal suture.

The jugal is long, flat and thin; it is gently curved to give the zygomatic arch its greatest width opposite the pterygoid flanges. The jugal is concave inside and convex outside, as in many mammals in which it is long. Its greatest height is at its posterior end as preserved ; in large specimens it measures $\mathrm{I} 2 \mathrm{~mm}$. here, with a tendency to increase in height posteriorly. The strongly compressed bone has a depressed anterior end. Anteriorly the bone loses 
height and also gets thicker and wider. The upper edge turns medially and after a few millimetres is covered by the posterior margin of the lateral flange of the lacrimal. An outer edge continues the general curvature of the bone and is covered by the antero-lateral margin of the lacrimal. The bone ends along the lateral wall of the infraorbital canal.

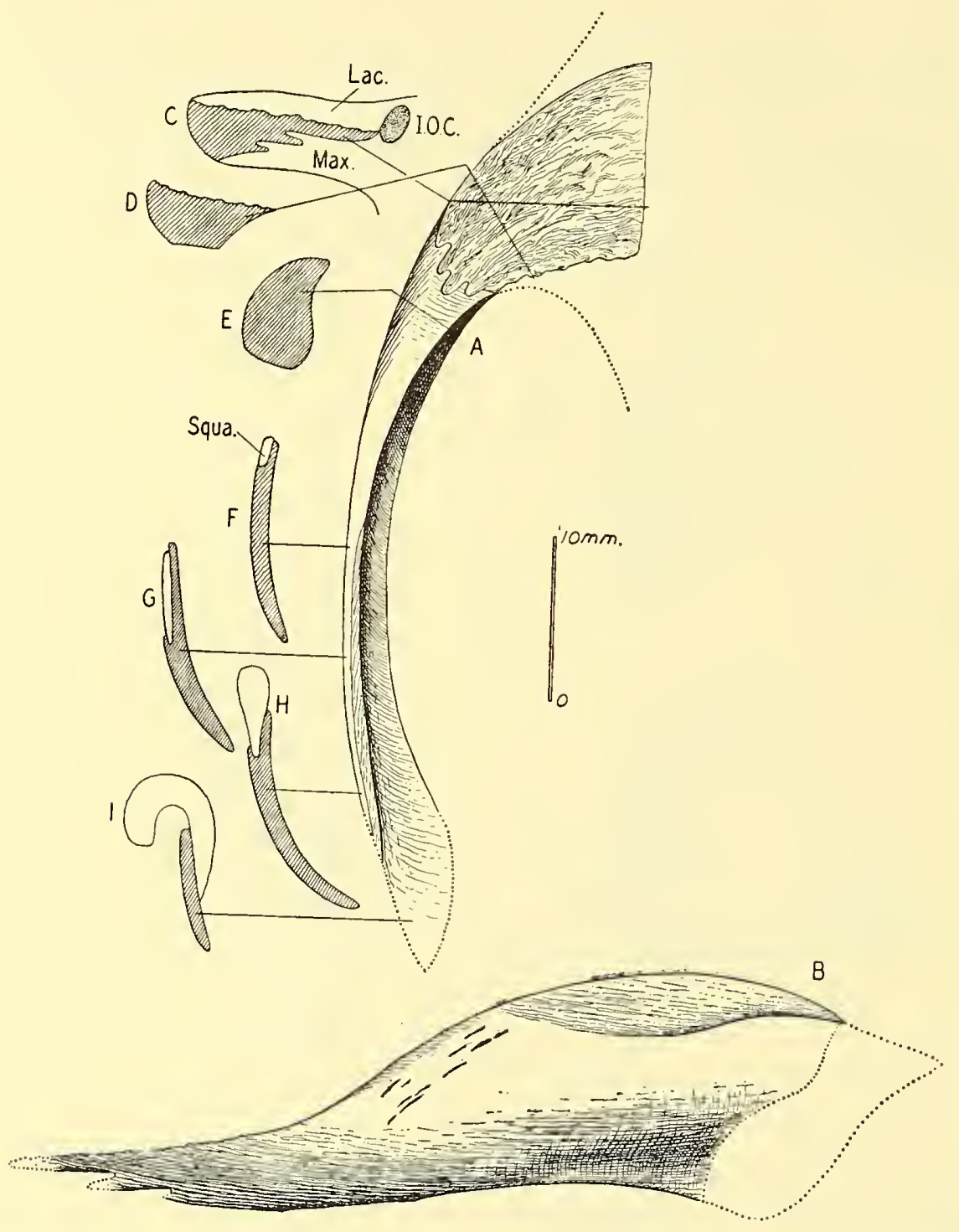

Text-Fig. 6.-The jugal of Oligokyphus. A, Dorsal view. B, Lateral view. C-I, Transverse sections. I.O.C., infraorbital canal ; Lac., lacrimal ; Max., maxillary ; Squa., squamosal.

The sutural area for the lacrimal is very rough and that for the inferior lateral flange of the maxillary is smooth. R.707I shows the feature mentioned when dealing with the jugular sutural area of the maxillary: two horizontal blades interdigitating with similar blades on the maxillary (R.7032).

Taken as a whole, the jugal has undergone profound changes from a Cynodont condition to a Tritylodont condition. In Cynodonts the squamosal ends anteriorly in a deep cleft on the upper margin of the jugal. In Oligokyphus a mere remnant of this arrangement is to be 
seen : the anterior end of the squamosal covers the upper part of the jugal externally, but the posterior half of the lower margin of this sutural area, as preserved in R.7075, is fractured at the end, indicating that a thin lamina of jugal was present lateral to the squamosal, and a trace of this lamina is actually preserved. The longitudinal surface markings of the sutural area with the squamosal are not parallel with the surface markings on the uncovered part of the bone. What has happened is that the markings of the squamosal have become impressed on the jugal.

In the triangular skull of Cynodonts the most exposed and widest parts are its posterolateral corners, formed by the jugal and squamosal, which are here very strong and massive. In Oligokyphus the skull is widest in the middle of the rounded zygomatic arch and the contact of jugal and squamosal has undergone great changes which will be discussed later. The posterior end of the jugal-on negative evidence-is not massive and strong, but exceedingly thin and weak. Indeed evidence regarding the posterior end of the jugal is provided by the squamosal and will be discussed when dealing with that bone.

The jugal of Cynodonts is distinguished by a ventral process from the lower margin, below the posterior rim of the orbit (Watson, I920:510). In Oligokyphus this process has disappeared. On the homologous place one can, however, detect a quite insignificant and hardly perceptible elevation on the otherwise perfectly smooth lower margin (R.7070).

The Cynodont jugal is characterized by its double anchorage anteriorly, with the postorbital and with the lacrimal plus maxillary, and by providing the posterior rim of the orbit. In Oligokyphus the postorbital has disappeared and with it any widening of the jugal behind the orbit.

In Cynodonts the contact of jugal with lacrimal and maxillary is vertical, comparable with the condition in polyprotodont marsupials; in Oligokyphus this contact is horizontal.

If one looks for an explanation of these significant changes at the anterior end of the jugal in Tritylodontids one can note the following: in Oligokyphus the distance between the occipital condyles and the pterygoid flanges has shrunk considerably compared with Cynodonts, and this is an essential change in the mammalian direction. But the jugal has counteracted this shortening by moving its anterior insertion forward, lateral to the row of cheek teeth, so that its lower anchorage can no longer be maintained by clasping the posterior end of the maxillary; this is a disadvantage mechanically. As its superior anterior anchorage has gone altogether the jugal thus left would be as long as it is in Cynodonts, but devoid of a firm insertion anteriorly. The lateral flanges of the maxillary and of the lacrimal, flanking the jugal between them, provide this safe insertion.

\section{THE DENTARY}

(Plate 4 ; Text-fig. 7)

This is the most numerously represented bone in the collection, more than eighty specimens being available. In order to include as many specimens as possible for comparison, the height and the breadth of the horizontal ramus at the anterior end of the masseteric fossa have been taken as dimensions. Only those teeth have been included under column four which are now present and erupted. Unerupted teeth, as well as those represented only by their roots or alveoli or the indication of a tooth formerly present because of pressure wear on the anterior face of an existing tooth, have been neglected in this count. 


\begin{tabular}{|c|c|c|c|}
\hline $\begin{array}{l}\text { Number of } \\
\text { specimen }\end{array}$ & $\begin{array}{l}\text { Breadth } \\
(\mathrm{mm} .)\end{array}$ & $\begin{array}{l}\text { Height } \\
(\mathrm{mm} .)\end{array}$ & $\begin{array}{l}\text { Number } \\
\text { of teeth }\end{array}$ \\
\hline R.7II9 & $6 \cdot 3$ & II $\cdot 8$ & 5 \\
\hline R.7I2I & $6 \cdot 4$ & II $\cdot 7$ & 5 \\
\hline R.7I 22 & $7 \cdot \mathrm{I}$ & II $\cdot O$ & 3 \\
\hline R.7I24 & $4 \cdot 5$ & $6 \cdot 0$ & o \\
\hline R.7I29 & $6 \cdot 3$ & IO $\cdot O$ & 3 \\
\hline R.7I30 & $4 \cdot 9$ & $7 \cdot 7$ & 5 \\
\hline R.7I3I & $6 \cdot 0$ & II $\cdot 4$ & o \\
\hline R.7I33 & $6 \cdot 8$ & II $\cdot O$ & 3 \\
\hline R.7I34 & - & IO 9 & 4 \\
\hline R.7I36 & $6 \cdot 5$ & $\mathrm{IO} \cdot \mathrm{O}$ & I \\
\hline R. 7 I 48 & $7 \cdot 0$ & II $\cdot 4$ & O \\
\hline R.7I49 & $6 \cdot I$ & II $\cdot 4$ & 2 \\
\hline R.7I $5^{\circ}$ & $4 \cdot 7$ & $7 \cdot 7$ & 2 \\
\hline R.7I5I & $5 \cdot 7$ & $9 \cdot 0$ & O \\
\hline R.7I54 & $7 \cdot I$ & $I 2 \cdot 6$ & $\mathrm{O}$ \\
\hline R.7I55 & $4 \cdot 8$ & $7 \cdot 9$ & 2 \\
\hline R. 7 I 56 & $4 \cdot 7$ & $7 \cdot 6$ & 3 \\
\hline R.7I57 & $4 \cdot 8$ & $7 \cdot 0$ & 4 \\
\hline R.7I $5^{8}$ & $4 \cdot 7$ & $7 \cdot 5$ & 4 \\
\hline R.7I60 & $4 \cdot 7$ & $7 \cdot 0$ & 2 \\
\hline R.7I6I & $4 \cdot 5$ & $7 \cdot 6$ & 3 \\
\hline R.7I62 & $4 \cdot 7$ & $7 \cdot 0$ & 2 \\
\hline R.7I63 & $4 \cdot 3$ & $6 \cdot 2$ & o \\
\hline R.7I64 & - & $7 \cdot 7$ & 3 \\
\hline R.7I65 & $5 \cdot 0$ & $8 \cdot 0$ & 2 \\
\hline R.7I67 & $4 \cdot 0$ & $5 \cdot 0$ & $\mathrm{O}$ \\
\hline
\end{tabular}

\begin{tabular}{|c|c|c|c|}
\hline $\begin{array}{l}\text { Number of } \\
\text { specimen }\end{array}$ & $\begin{array}{l}\text { Breadth } \\
(\mathrm{mm} .)\end{array}$ & $\begin{array}{l}\text { Height } \\
\text { (mm.) }\end{array}$ & $\begin{array}{l}\text { Number } \\
\text { of teeth }\end{array}$ \\
\hline R.7I68 & $3 \cdot 8$ & $4 \cdot 8$ & $\mathrm{o}$ \\
\hline R.7I69 & $3 \cdot 2$ & $4 \cdot 3$ & I \\
\hline R.7I7I & $3 \cdot 4$ & $5^{\cdot} \mathrm{O}$ & o \\
\hline R.7I72 & $3 \cdot 6$ & $5 \cdot 4$ & o \\
\hline R.7I73 & $5 \cdot I$ & $7 \cdot 0$ & 2 \\
\hline R.7I75 & $4 \cdot 8$ & $7 \cdot 2$ & 2 \\
\hline $\mathrm{R} .7176$ & $4 \cdot 5$ & $6 \cdot 9$ & 2 \\
\hline R.7I77 & $5 \cdot 0$ & $7 \cdot 3$ & I \\
\hline R.7I8I & $6 \cdot I$ & II $\cdot 2$ & 4 \\
\hline R.7I82 & $5 \cdot 7$ & $9 \cdot 3$ & o \\
\hline R.7I83 & $5 \cdot 7$ & $9 \cdot 5$ & 2 \\
\hline R.7I84 & $5 \cdot 3$ & $8 \cdot 9$ & o \\
\hline R.7I88 & $3 \cdot 2$ & $4 \cdot I$ & o \\
\hline R.7204 & $4 \cdot 2$ & $6 \cdot 2$ & 4 \\
\hline R. 7250 & $6 \cdot 9$ & $\mathrm{I} 2 \cdot 5$ & o \\
\hline R.7252 & $2 \cdot 6$ & $3 \cdot 6$ & o \\
\hline $\mathrm{R} .7253$ & $5 \cdot 0$ & $7 \cdot 8$ & 3 \\
\hline Copenhagen spec. & L. $6 \cdot 7$ & I3 $\cdot I$ & 2 \\
\hline S. Dakota S.o.M. & $6 \cdot 3$ & $\mathrm{IO} \cdot 2$ & 2 \\
\hline Basle N.H.M. & $6 \cdot 0$ & $9 \cdot 0$ & 5 \\
\hline W.G.K.3 & $4 \cdot 5$ & $6 \cdot 9$ & o \\
\hline W.G.K.6 & $5 \cdot 3$ & $8 \cdot 5$ & 2 \\
\hline W.G.K.I3 & $4 \cdot 4$ & $7 \cdot 7$ & o \\
\hline
\end{tabular}

And 39 other specimens which have not been measured.

Almost the whole of the dentary is known. In outline, the lower border of the horizontal ramus is evenly and slightly convex, from the angular corner to the anterior end of the symphysis. Its deepest point lies below the first cheek tooth. There are two small interruptions in this curve; the anterior one is the pronounced posterior part of the symphysis, the posterior one is a very slight indentation on the medial side of the bone, beginning at the lower border below the proximal end of the fifth cheek tooth and slanting upwards and posteriorly ending at or behind the dental foramen. This indentation is rather slight in many specimens, but a very reliable and constant character is the narrowing of the bone behind it up to the angular corner. This corner is homologous with the angular process of mammals, but as it is never a sharp-angled projection, being either obtuse-angled or rightangled, it is not a process sensu stricto.

From the angular corner to the tip of the articular process, the outline of the bone continues at first in a concave, and then for a posterior third of this distance in a slightly convex, border. From the posterior end of the articular process to the highest point of the coronoid process the outline is concave, but as the superior part of the coronoid process is the only part of the dentary which has not yet been found, its further curvature is unknown. 

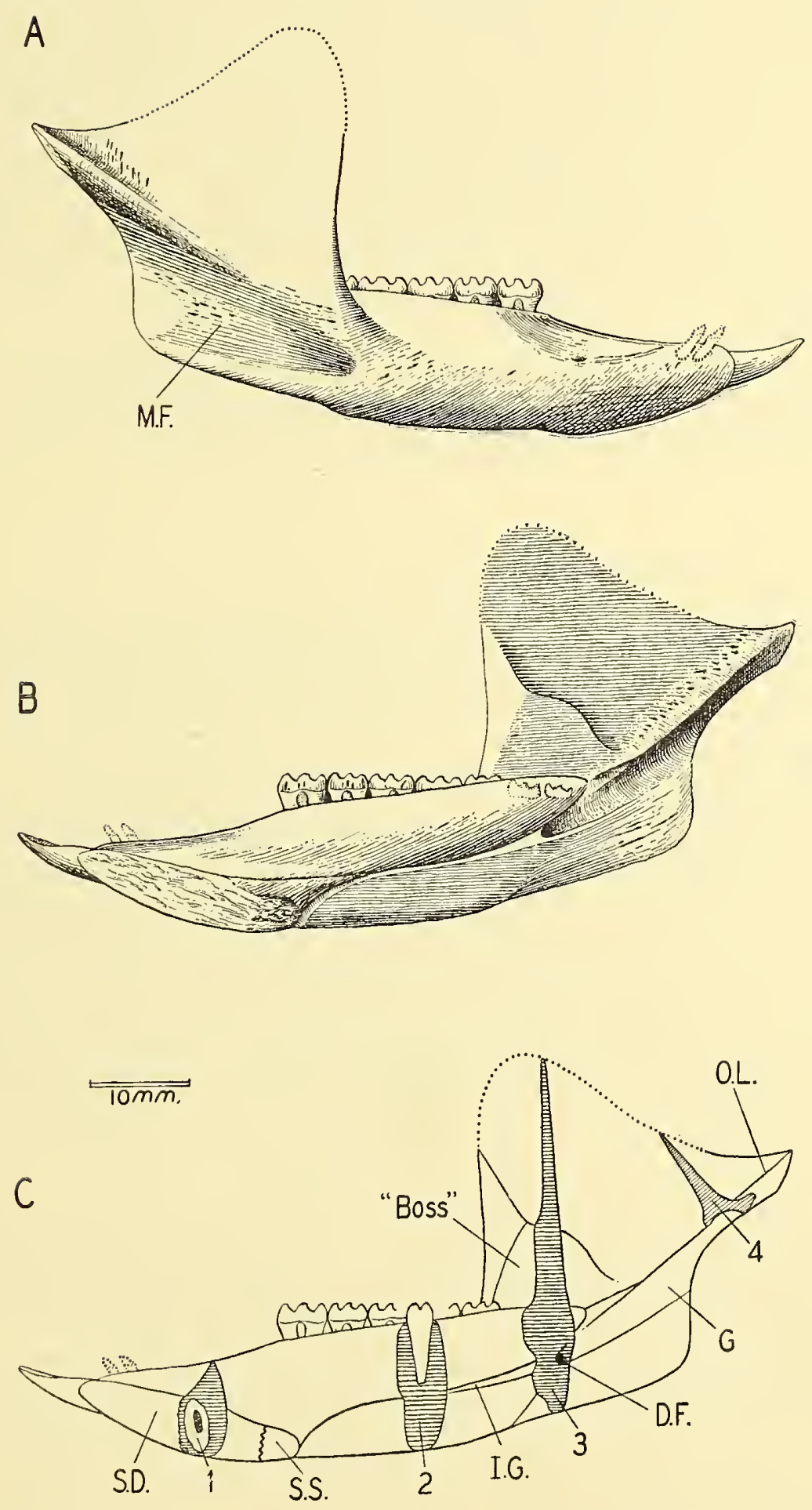

Text-Fig 7.-The dentary of Oligokyphus. A, Lateral view ; M.F., masseteric fossa. B, Medial view. C, Key for medial view and superimposed four transverse sections. D.F., dental foramen ; $G$, groove for accessory bones of lower jaw; I.G., internal groove; O.L., overhanging ledge ; S.D., symphyseal sutural surface for dentary ; S.S., sutural surface for splenial ; "Boss", in approximation to the lateral surface of the flange of the pterygoid. I-4, Transverse sections. 
The alveolar border, straight from the last to the first cheek tooth, becomes rather sharp with the beginning of the diastema, its profile distinctly concave and uninterrupted by the small alveoli for $I_{2}$ and $I_{3}$. The two rows of cheek teeth diverge very slightly posteriorly. As the two rami of the lower jaw diverge at an angle of $30^{\circ}$, the straight row of cheek teeth passes medially of the ascending ramus in such a way that the sixth and seventh cheek teeth and even half of the fifth cheek tooth are hidden behind the ascending ramus when viewed laterally. In very small individuals (R.7I67 and R.7I68) where the basicranial width is relatively much greater than in the large forms, the rami of the lower jaw diverge much more and the oblique setting of the row of cheek teeth on the horizontal ramus is therefore much more pronounced.

The ascending ramus begins some millimetres below the anterior end of the fourth cheek tooth and about $4 \mathrm{~mm}$. lateral of the tooth-row ; it rises obliquely to a point $3 \mathrm{~mm}$. above the middle of the fifth cheek tooth and continues almost vertically.

\section{Medial Side of the Dentary (Text-fig. 7 в and c)}

The posterior end of the alveolar border of the horizontal ramus develops a blunt, prominent corner protruding medially and distinctly divided from the posterior part of the dentary. The dental foramen is situated below this prominent corner which in the large species contains an unerupted cheek tooth.

Rising from the dental foramen and from the upper posterior point of the prominent corner mentioned above, there are two converging edges which include between them a small concave area, triangular in shape. As this area leads directly into the dental foramen it is most likely that it indicates the course of the mandibular branch of the $\mathrm{N}$. trigeminus. The lower of the two edges is continued as a wide overhanging ledge, which ends only at the posterior end of the dentary. From the dental foramen issues another edge, parallel with the first, ending above the angular corner at a point which is, in mammalian terminology, inside the subcondylar notch. Thus between this edge and the overhanging ledge there is a wide and spacious groove, which has its counterpart in Cynodonts where it houses the accessory bones of the lower jaw. As it approaches the posterior end of the articular process of the dentary, the underside of the overhanging ledge is no longer concave, but plane. Immediately before the tip it narrows very much, forming with the masseteric ridge on the lateral side of the bone the end of the articular process which is horizontal, lancet-pointed and certainly not bluntly conical (R.7I26). There is not the slightest doubt that this point as described and figured is the natural end of the dentary and that nothing of the bone is missing in this region. It is certain that this point never served as a condyle for the dentary. The superior border of the overhanging horizontal ledge is continued anteriorly by an obtuse-angled ridge, which curves upward in a slight double-bend and divides the thin postero-superior part from the thick and massive antero-inferior part of the coronoid process. This latter part has its much more pronounced homologue in Bienotherium and Broom's Ictidosaur "A " where it has been called " the boss" (Watson I942). There is no evidence of the presence of a coronoid and this bone must be considered as absent in Oligokyphus.

The internal groove: below the ridge which terminates the groove for the accessory 
bones of the jaw runs a shallow and narrow but distinct groove (R. 7I2I, R.7I22, R.7I30) which continues below the dental foramen and ends with a distinct downward bend at the posterior end of the symphysis (R.7I22, R.7I40, R.7I42). It seems that the vessel which was lodged in this groove emerged at the front of the symphyseal area. From the lateral side of the dental foramen branches a distinct but very narrow groove, which can be followed anteriorly to a region above the posterior two-fifths of the symphysis. The course of this narrow groove is always above the wider groove just described. The dual origin (from the ridge below the angular groove and from the dental foramen) and the dual course at the symphysis (above and below it) make the internal groove of Oligokyphus very similar to the same structure described by Simpson (I928a) in Mesozoic mammalia. His drawing of the condition of Docodon (fig. 3) is actually identical with the condition found in Oligokyphus. In none of the Oligokyphus material is the symphysis of the lower jaw co-ossified. Further information about the symphysis will be found under the heading Splenial.

\section{Lateral Side of the Dentary}

In adult individuals one mental foramen is situated just below the anterior end of the first cheek tooth. The masseteric fossa is distinct and large; its borders are never sharp but are confluent with the horizontal and ascending ramus. A strong buttress reaches half-way forward into the masseteric fossa issuing from the tip of the articular process, at which point it is most prominent. On top the buttress is flat, on the underside it is gently convex, not concave like the overhanging ledge on the medial side of the bone.

The absence of a condyle on the dentary, and the wide groove which is homologous with the same structure in Cynodonts where it houses the accessory bones of the jaw, make the conclusion inescapable that the lower jaw of Oligokyphus is compound. The articulation of the jaw was by means of an articular and a quadrate.

The position of the ginglymus between articular and quadrate in relation to the dentary can be established; it must have been immediately behind the posterior end of the dentary. In the large form that would be $8-9 \mathrm{~mm}$. above a plane formed by the cusps of the lower cheek teeth, approximately $30 \mathrm{~mm}$. behind the dental foramen, and $\mathrm{I} 2 \mathrm{~mm}$. from the sagittal plane, each ramus of the lower jaw diverging from this plane at an angle of $15^{\circ}$.

\section{THE SPLENIAL}

There is no specimen of a splenial and evidence for the bone is indirect and from the dentary. In Cynodonts the splenial reaches from the posterior part of the symphysis to the dental foramen: the splenial of Oligokyphus, as will be shown, does the same. Specimens R.7I2I, R.7I22, R.7I4O show the symphysis very well. It is apparent that the posterior fifth of the symphysis makes an obtuse angle of about $160^{\circ}$ with the anterior portion. As the whole of the symphysis has the characteristic surface that indicates bone contact, one is forced to the conclusion that the posterior fifth of the symphysis was in contact with a bone which could not have been the dentary. This can only have been the splenial. Below the internal groove, from the dental foramen to the symphysis, the horizontal ramus is noticeably flat (R.7I22, R.7I39, R.7I54). The same condition is met with in Diademodon, where this flattened area is the contact-surface for the splenial. These two lines of evidence are sufficient for the existence of the splenial in Oligokyphus to be deduced. 
It is convenient to mention here some points which are not entirely germane to this monograph : Simpson (I928a) made a very interesting study of a structure, which he aptly called the internal groove, on the dentary of Mesozoic mammalia. But despite his numerous observations, he did not find it possible to show why the internal groove is a constant element in Jurassic mammalia and why it is lost in Tertiary mammals.

A consideration of the structural forerunners of mammals may provide the key to this problem: Cynodonts have a well-developed splenial as well as an internal groove, and Oligokyphus has the same. Wherever a vessel or a nerve is situated between two bones, these bones have to be grooved to accommodate the soft structure between them; hence the existence of the internal groove in Cynodonts and in Oligokyphus can be explained by the presence of a splenial in these forms. Since we find the internal groove as a constant character in Jurassic mammalia, we can postulate the existence of the splenial in these animals with a high degree of probablility. Moreover the Multituberculates, which are not found before the Upper Jurassic and are seemingly the latest order of mammals to appear in the Jurassic, have no internal groove, whereas the Triconodonts and Panthotheria both have it. The Symmetrodonts possess an internal groove too and are now known from the Rhaeto-Liassic (Kühne, I950a).

As the splenial has no relation to the condylar region of the dentary, it is quite possible that it was still in existence when the other accessory bones of the lower jaw had either disappeared or had taken over a new function in the middle ear. This condition would apply in the Jurassic mammalia with the exception of the Multituberculates. Once the splenial had disappeared, vessel or nerve or both, no longer confined to the narrow canal between dentary and splenial, could run between the muscles and fasciae of the lower jaw and any impression of them on the medial side of the dentary would be the exception rather than the rule. Such a condition would apply to Recent and Tertiary mammalia. If the existence of the splenial can be proved in Jurassic mammals, the most important osteological character on which to distinguish Reptilia and Mammalia--the compound or simple nature of the lower jaw-will lose much of its value.

\section{IDENTIFICATION OF ARTICULAR AND QUADRATE}

The Cynodont articular-quadrate joint forms a perfect ginglymus, the quadrate providing the trochlea and the articular the articular notch. Hence, if two bones are found which form a ginglymus and which cannot be accounted for as any other part of the skeleton, they may have formed the primary articulation of the lower jaw of Oligokyphus. Several such specimens have been found.

In the later Cynodonts, Cynognathus and Diademodon, the short articular is always co-ossified with the prearticular medially and the surangular superiorly. Granted similar conditions in Oligokyphus, the ensuing bone-complex would be rather elongated and tapering anteriorly. The bone provisionally identified as the articular complex fulfils these requirements. The bone provisionally identified as the quadrate has one long peg-like process at right angles to the axis of the trochlea; as the bone is complete, and if the identification of the bone is correct, this means that in Oligokyphus the quadrate and the quadrato-jugal are still separate bones, which is at variance with the condition of later Cynodonts. In Cynognathus and Diademodon, for instance, the squamosal has always two notches and the quadrate-quadrato-jugal complex always has two prongs which fit into these notches. 


\section{ORIENTATION OF THE ARTICULAR COMPLEX AND THE QUADRATE}

As bones are available from both right and left sides, there are many possible positions and combinations. The position of the bones when a complete fit of the two articular surfaces is achieved suggests itself as the correct one. Moreover their relation to other bones of the skull is the natural one in this position and in some cases the only possible one. The anteroposterior orientation of the articular complex is determined by the articular notch, which is at the posterior end. If the articular complex together with the angular is ensheathed by the groove for the accessory bones in the dentary, the direction of this groove-inclined $30^{\circ}$ forwards and converging toward the sagittal plane at $15^{\circ}$ - should be more or less identical with the direction of the longitudinal axis of the articular complex. The rotatory axis of the articular notch in the articular makes an angle of $30^{\circ}$ (or $\mathrm{r}_{50^{\circ}}$ ) with the longitudinal axis of this bone. Assuming a transverse position for the ginglymus of quadrate and articular, such as occurs in Cynodonts, the medial side of this bone would be where the acute angle $\left(30^{\circ}\right)$ is formed between the rotatory and longitudinal axis and anterior to the articular notch.

If the superior and inferior side of the articular complex could be determined, right and left bones could be identified and a quadrate which fitted would be orientated and identified automatically.

The surface of the groove for the accessory bones in the dentary is concave and smooth and any bone in contact with it must be supposed to have a corresponding surface similarly smooth and convex. The contact of prearticular and angular is a normal sutural one and the contact surface can be expected to be of rather high relief. The bone provisionally identified as the articular complex has two kinds of surfaces along its shaft, one convex and smooth, the other deeply grooved longitudinally. Both surfaces are anterior to the articular notch and either above or below it, according to the orientation of the bone. Since it may be assumed that surangular and prearticular join in the formation of the articular complex, the surangular surface, which is smooth and convex, will be that in contact with the groove in the dentary, and will be the superior, whereas the deeply grooved surface will be the inferior, providing the contact surface for the angular on the prearticular.

\section{DESCRIPTION OF THE ARTICULAR COMPLEX}

(Plate 5, figs. I, 2, 4 ; Text-fig. 8)

Material: There are six specimens available, R.7189-94. Principal specimens are R.7I89 (left), from a large animal, and R.7Igo (right), also from a large animal.

No distinct sutures can be made out on any specimen. The bone is described in the orientation defined above. The articular notch occupies $140^{\circ}$ of a cylindroid surface, which ends above in a well-defined lip and below in a rather straight margin. There is no retroarticular process. This margin is rounded at its medial end (R.7Igo) and laterally it is drawn out into an acute point. Between the apex of the lip above and the acute point below, the lateral margin of the articular notch has a small but characteristic excavation. The articular notch is open medially. The surface of the notch is not absolutely cylindrical, for it bears a faint and slightly lateral convexity which is distinct towards the lip of the 
articular notch but fades out towards the lower margin of the notch. On the medial side of the bone and directly antero-inferior to the articular notch there is a prominent bulbus with the characters of a muscle insertion. On the lateral side the contact of articular and surangular is well shown in Text-fig. 8, D. This contact agrees with the condition in Cynognathus sp. drawn by Broom (I904, pl. 35, fig. 5) and thus corroborates the suggested orientation of the bone.

The muscle insertion must be for part of the M. pterygoideus internus. The position of the insertion is as required for this muscle and its presence here corroborates the orientation of the articular complex. Furthermore, the muscle insertion is additional evidence

A
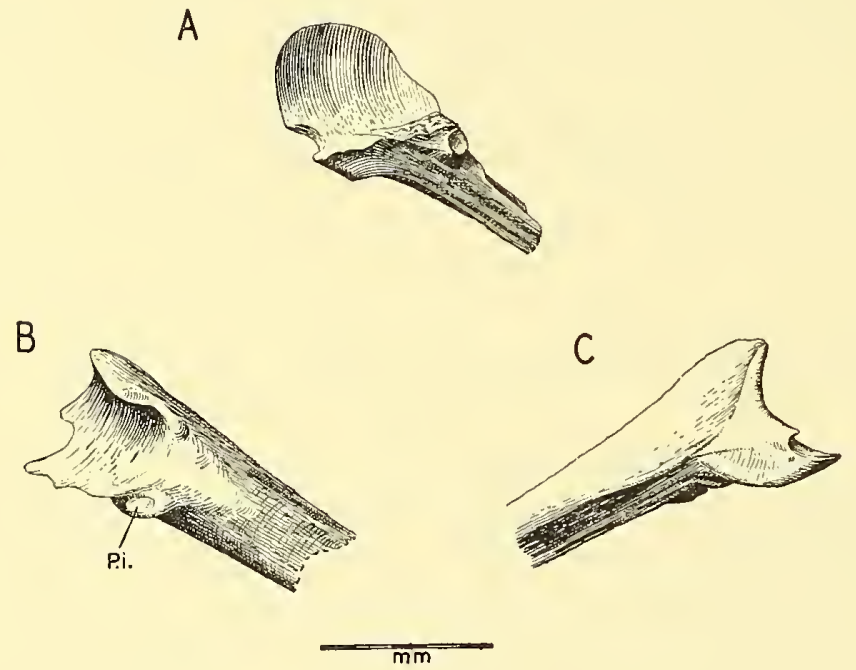

D

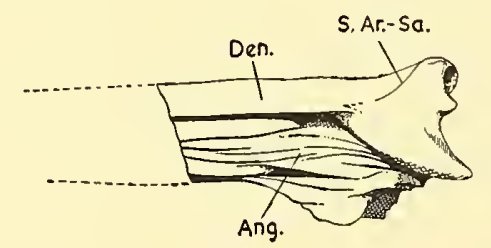

TeXt-rig. 8.-Articular complex of Oligokyphus. A, Posterior view. B, Internal view. C, External view. D, View from below and laterally. P.i., insertion area for M. pterygoideus internus ; $A n g$., sutural surface for angular; Den, surface which is covered by the dentary ; S.Ar.-Sa, supposed suture between articular and surangular.

for the articular-malleus homology. The insertion for part of the M. pterygoideus internus in this bone is exactly at the position where in mammalia the M. tensor tympani inserts on the malleus.

To summarize this account of the accessory bones of the jaw : the coronoid is lost in Oligokyphus; its place and function have been taken by an outgrowth of the ascending ramus of the dentary. The splenial, though it exists, is not in the material studied. The angular too is not available; it is regarded as a separate and well-developed bone. The deeply furrowed inframedial surface of the articular complex was in contact with it. The articular is fused with the surangular and prearticular as in Cynodonts. 
THE QUADRATE

(Plate 5, figs. I, 4 ; Text-fig. 9)

Material: There are four specimens: R.7I95 is a complete left; R.7I96 is a right with complete trochlea; R.7I97 and R.7I98 are smaller fragments.

The trochlea covers about $280^{\circ}$; it shows a constriction, correlated with the slight convexity of the articular notch already mentioned. Antero-superiorly the trochlear surface has a rounded margin to accommodate " the lip " on the articular notch ; postero-superiorly the margin of the trochlear surface is almost straight. Laterally the trochlea is truncated, suggesting that a small quadrato-jugal was in contact here. R.7Ig6 shows that there was no foramen between the quadrate and the quadrato-jugal. The trochlea fills the articular notch in the articular completely, so that the quadrato-jugal did not take part in the articulation of the lower jaw (Parrington I946, figs. 5, 6).

A
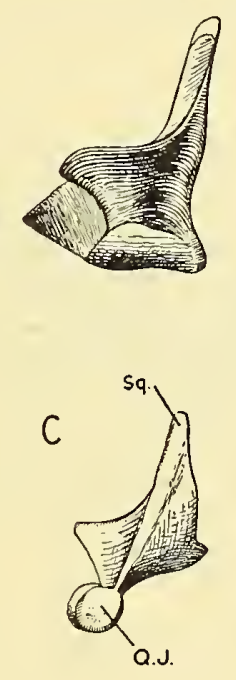

B

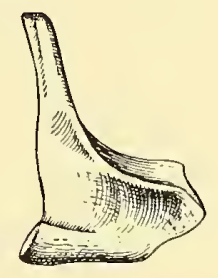

D

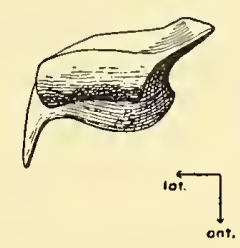

Text-Fig. 9.-Left quadrate of Oligokyphus. A, Frontal view. B, Posterior view. C, Lateral view. $S q$. ." "peg", articulating with squamosal; $Q . J$., surface on the trochlea, articulating with the quadratojugal. D, Ventral view.

Superiorly, a thin vertical and transverse sheet of bone supports the trochlea. This sheet is strengthened by a pillar-like thickening above the antero-medial part of the trochlea. The medial margin of this vertical sheet is drawn out into a process in a postero-medial direction. Watson (I9II) found a medial process on the quadrate in Diademodon polyphagus, directed antero-medially and in contact with the quadrate ramus of the epipterygoid. In the later Protacmon (Watson, I920) no such process is found on the quadrate. The same applies to Cynognathus and even to the early Thrinaxodon (Parrington, I946). Olson however mentions a stapedial process in Thrinaxodon (Olson, 1944). The process in Oligokyphus is probably a stapedial process. Its postero-medial direction does not suggest that it was in ligamentous contact with the epipterygoid.

The vertical and transverse sheet above the trochlea widens above. Its flat and smooth upper surface is confluent with the medial surface of the peg-like process which 
rises high on the lateral side of the bone and keeps contact with the squamosal at its extremity. As described by Parrington (I946) in Thrinaxodon, the upper end of the peg-like process of the quadrate ends abruptly as if cut off. In the only specimen of the region in question, the end of the peg-like process does not look as if it were broken off.

The flat dorsal surface of the quadrate has no corresponding articular surface on the squamosal and the same applies to the peg-like process at the lateral side of the quadrate. Ligaments must have maintained the connection between the quadrate and the squamosal, aiding the movement of the quadrate during mastication.

\section{SQUAMOSAL}

\section{(Plate 6; Text-fig. Io).}

Material: There are twenty specimens, R.7088-R.7107. The anterior end is lacking in all of them; eight specimens consist of the posterior part of the bone; the remainder are fragments of the zygomatic process.

No measurements are given, as it has not been possible to select a suitable standard measurement in the majority of specimens. The identification of the bone rests on R.709o, the largest cranial fragment in the collection, where the posterior part of a left squamosal is associated with a complete basicranium. The squamosal articulates with the lateral extremity of the paroccipital process by an oval depression. Above this is a groove, which is the upper half of a small canal, the post-temporal foramen. Above and medially of this foramen the squamosal forms a flat, thin sheet of bone which is applied to the widened lateral edge of the tabular and extends dorsally to touch the parietal. From this attachment the bone passes out laterally ; its upper surface, rounded from back to front; lies in a plane below its more dorsal extension on the brain-case.

The ventral surface of this part of the bone has a smooth depressed area in front, which ends in a deep ridge bounding a deeper and more extensive excavation for the attachment of the quadrate. More laterally the bone swings forward to lie just within the external surface of the head and to extend forward to meet the zygomatic process of the jugal in a long oblique overlap.

Even the most lucid description of a bone new to science is hardly illuminating. One way of conveying the characteristic features of the bone is to compare it with the same bone of an animal as nearly related as possible. The comparison in this case is with Bienotherium and Diademodon. In the latter, the pertinent structures are well known and have been illustrated sufficiently. Bienotherium is intermediate in time and, though a Tritylodontid, it is in many respects intermediate in structure.

Starting from the condition in Diademodon, two factors which govern the shape of the squamosal in Oligokyphus may be pointed out. Firstly, the squamosal in Cynodonts together with the jugal is essentially a structural unit, giving the box-like skull rigidity against stresses in its longitudinal axis. In Oligokyphus a greatly developed sagittal crest takes such stresses and makes a strong squamosal and jugal superfluous. Secondly, the breadth to length ratio of the skull is much smaller in Cynodonts than in Tritylodontids. The skull of Cynodonts (Diademodon) approaches an isosceles triangle in shape, with its base formed by the excessively long paroccipital processes and the greatly developed and 

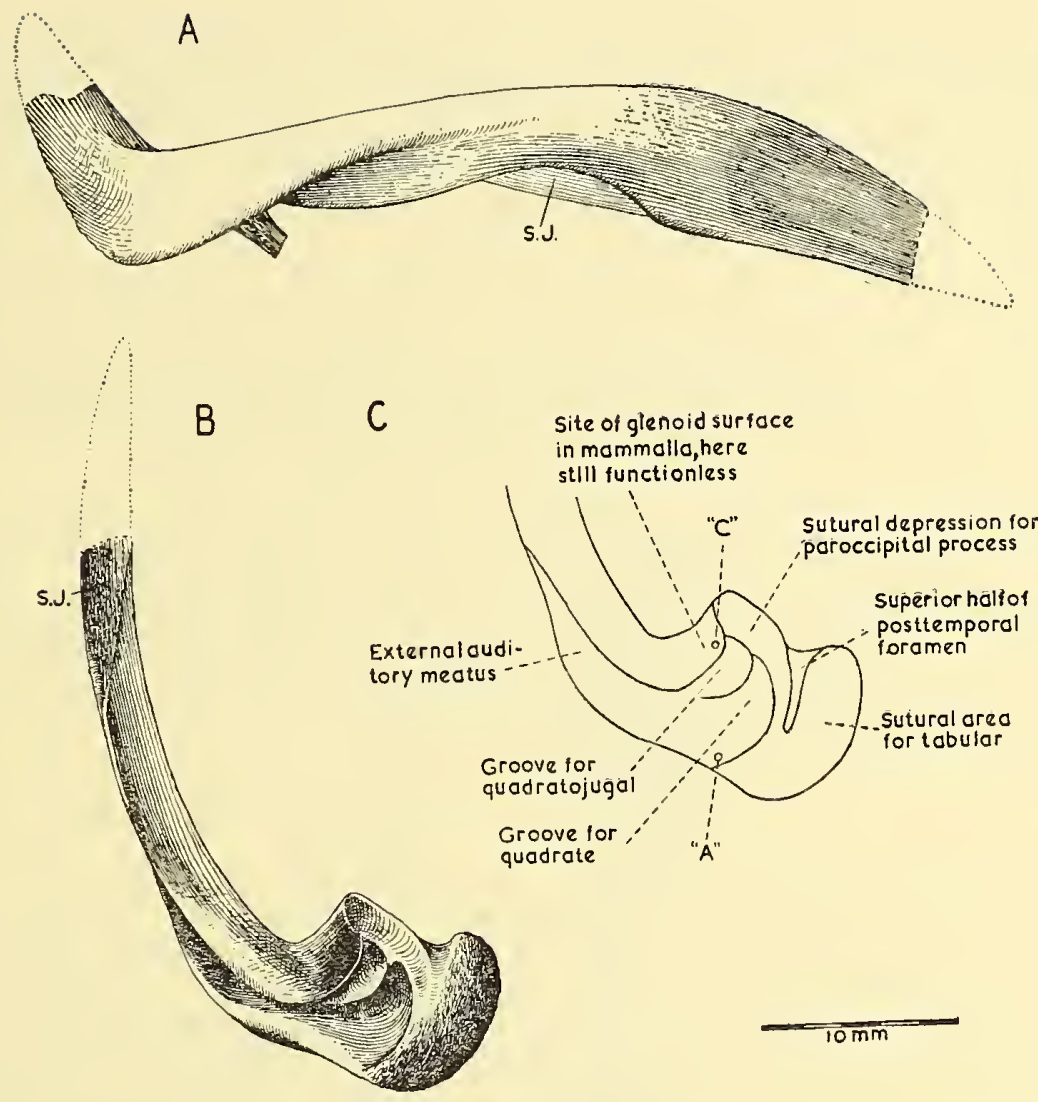

Groove lor ', "Á
quadrate

TEXT-Fig. IO.-Squamosal of Oligokyphus. A, Lateral view; B, Ventral view; S.J., sutural surface for jugal. C, Key for B.

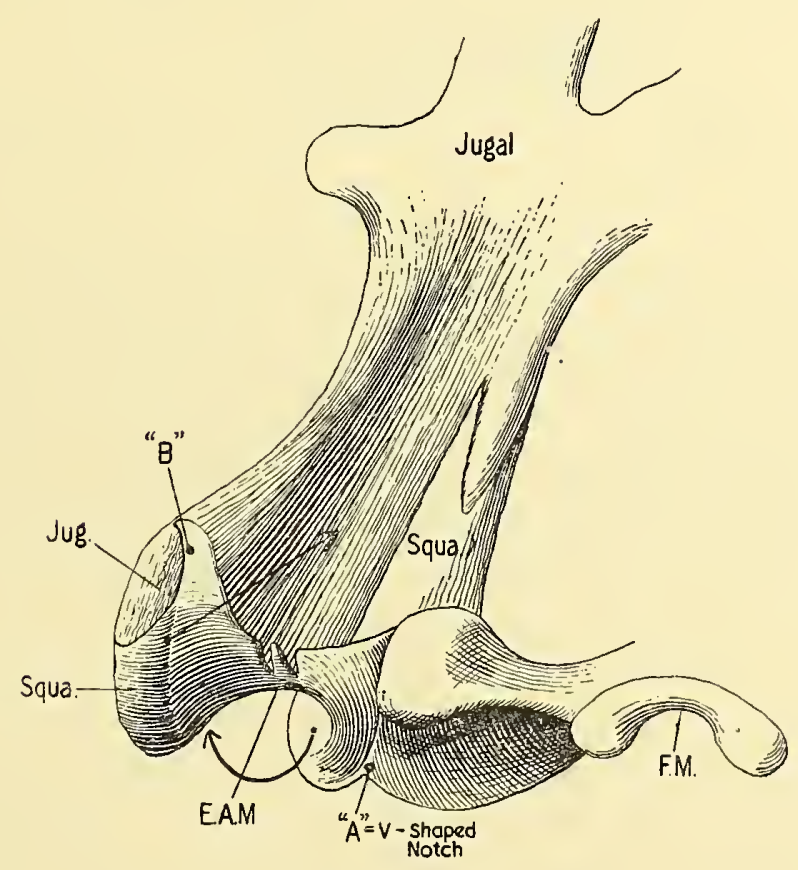

TEXT-FIG. II.-Zygomatic arch of Diademodon in ventral view. "A" and "B", reference points, see text. The two arrows indicate the movement that parts of the squamosal would have to perform to arrive at a Tritylodont condition. E.A.M., external auditory meatus ; F.M., foramen magnum. 
produced postero-lateral ends of jugal and squamosal. In Oligokyphus the length of the paroccipital process is reduced and so is the width of the external auditory meatus. The greatest breadth of the skull is across the zygomatic arches. Both these factors make a considerable reduction of the squamosal.

I. The V-shaped notch.

Diademodon: between the medial plate of the squamosal, which is in contact with the tabular, and the medial rim of the external auditory meatus there is a deep and steep $V$-shaped notch, the two sides of which enclose an angle of $50^{\circ}$. The zygomatic process rises nearly to the level of the sagittal crest.

Bienotherium: the V-shaped notch is identical with that in Diademodon. The sagittal crest rises slightly above the level of the zygomatic arches. (See Text-fig. II).

Oligokyphus: as the height of the squamosal is reduced, the V-shaped notch is shallow and wide, including an angle of $100^{\circ}$. The zygomatic arch rises very little above the bottom of the $\mathrm{V}$-shaped notch.

II. The external auditory meatus.

Gregory's tentative identification (IgIo) of the structure dealt with below as the external auditory meatus seems to be correct. In no living or fossil mammal is there a groove in the squamosal comparable with that in Cynodonts. Be this as it may, the evidence furnished by Oligokyphus corroborates Gregory's identification. The course of the cartilaginous external auditory meatus in Ornithorhynchus is similar to the course of the groove in the squamosal in Oligokyphus and the diameter of this groove, leading forward from the tympanic region, is so narrow in Oligokyphus that it would only allow a meatus of mammalian dimensions to pass through it.

Diademodon: the external auditory meatus is a groove, opening posteriorly and laterally. It is extremely wide, owing to the postero-lateral rim being extended laterally.

Bienotherium: the posterior part of the left zygomatic arch is preserved. It provides evidence of the great width of the zygomatic arch in this region, as in Cynodonts. As the skull is imperfectly preserved and lacks sutures, the posterior end of the jugal cannot be seen but it is probably as shown in Text-fig. I2. On this assumption the prominent squamoso-jugular corner of Cynodonts (point " B ") can be homologized with its counterpart in Bienotherium. Here it has migrated medially, leaving the medial rim of the external auditory meatus as the most lateral structure of the posterior part of the skull. The medial rim of the meatus remains unchanged and in a Cynodont condition.

Oligokyphus : the external auditory meatus is a narrow groove which is open only from below. This is due to a change in the condition found in Bienotherium: the medial rim has developed outward and downward and has increased relatively to the condition in Cynodonts; the lateral rim, however, is essentially unchanged. The result of this development is that the postero-lateral corner of the Cynodont skull, formed by the squamosal and jugal (point " B" in the figures) no longer exists in Oligokyphus. The lateral rim of the external auditory meatus in Cynodonts has become 
the medial rim of this structure in Oligokyphus and the medial rim of the external auditory meatus in Cynodonts has becorne the lateral rim of this structure in Oligokyphus. From now on, this Cynodont terminology must be abandoned and the rims of the external auditory meatus of Oligokyphus called lateral or medial according to the position they occupy in this genus. For descriptive purposes the roof of the external auditory meatus may be taken in a caudal direction. The lateral rim of the external auditory meatus begins in the middle of the squamosal and gains continuously in depth until it comes to an end at point " A." The edge of this rim is rounded. The lateral rim is evenly concave except for the last $5 \mathrm{~mm}$. where the curvature becomes convex.

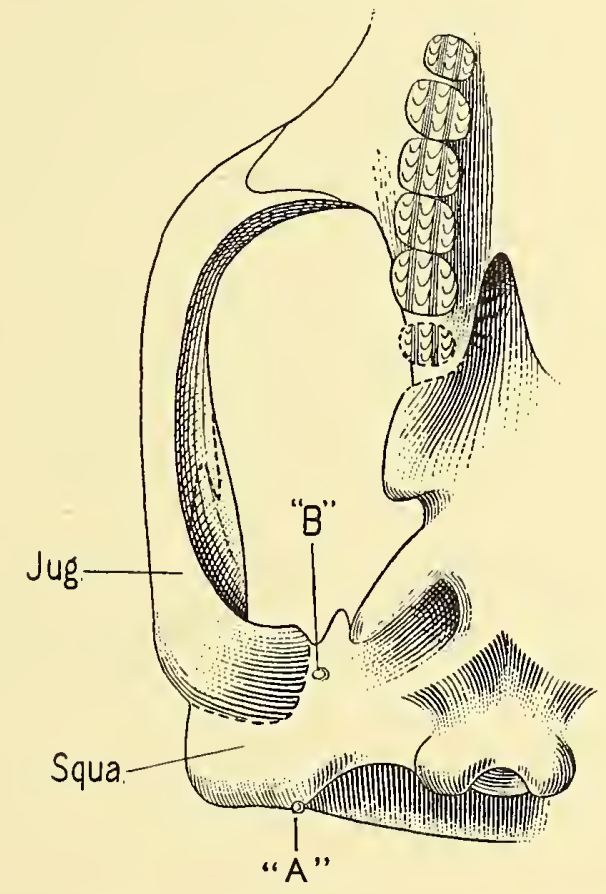

TEXT-FIG. I2.-Bienotherium yunnanense. Interpretation of the sutures of the jugal.

The medial rim of the external auditory meatus is identical with the continuous lower margin of the zygomatic process ; its edge along its whole course is rather sharp and the medial wall of the meatus is notably flat, as if a bone were in contact with it. As in Bienotherium, such a bone could only have been the jugal (but see below). The course of the medial rim is at first parallel with the sagittal axis, then it becomes turned at about $15^{\circ}$ towards the sagittal plane and finally curves round in a wide $\mathrm{U}$ until its end is almost parallel with its beginning. In its middle and anterior course, the meatus is not wider than I $\mathrm{mm}$. but its posterior part widens into a large quadrant-shaped basin.

III. The articulation of quadrate and quadrato-jugal and other features of the posterior part of the squamosal.

In Cynodonts and Diademodon the inferior rim of the external auditory meatus is an edge pointing downward and orientated at right angles to the sagittal plane. It 
carries two notches, the lateral one deeper than the medial. In Thrinaxodon the lateral notch receives the quadrato-jugal, the medial receives the quadrate. Both these bones tend to drop out after death and are rarely found in situ. It is observable that surfaces of the pegs of the quadrate-quadrato-jugal complex which are in contact with the squamosal grooves do not show structures guaranteeing a close fit, such as a ridge on one surface fitting into a groove in the other. Medially of the two notches, the thin edge of the squamosal is drawn out into a short but distinct spout, pointing downwards and medially and ending on the infero-lateral face of the long and massive paroccipital process. Above this spout, and medially of the extensive contact between the squamosal and the paroccipital process, is situated the post-temporal foramen. Seen from behind this foramen perforates the tabular; on the inside of the temporal fossa, it is seen to emerge between the squamosal and the paroccipital process, both bones providing one half of its outlet. Medially and dorsally of the post-temporal foramen the medial sheet of the squamosal has extensive contact with the tabular.

Bienotherium: one result of the medial migration of the jugal-squamosal corner (point " B ") is its approach to the paroccipital process and the concomitant backward displacement of the quadrate cum quadrato-jugal, away from the inferior rim of the external auditory meatus. The area for the articulation of the quadrate in Bienotherium has to be looked for about $2 \mathrm{~mm}$. posterior to the lower end of the bar, leading to the letter $\mathrm{Q}$ in Young's text-fig. 3 (I947). Neither the articular area for the quadrate nor the quadrate itself is preserved in Bienotherium.

Oligokyphus: the ventral surface of the posterior part of the squamosal shows the narrow groove of the external auditory meatus, situated antero-laterally with its two rims diverging medially and enclosing a quadrant-shaped depression with one corner at "A". A line connecting " $\mathrm{A}$ " and " $\mathrm{C}$ ", which is at an opposite corner, is nearly parallel with the sagittal axis. The outer rim of the external auditory meatus continues to "A". From "A" in an antero-medial direction the depressed area is enclosed by a steep and thick wall, which forms medially the contact area of the squamosal with the tabular (R.7089). This margin continues antero-laterally, as a thin lamina of bone dividing the depressed area from an oval concavity anteromedial to it, which receives the anterior part of the paroccipital process (R.7090). Owing to its extreme thinness, the full height of this lamina is not preserved in any specimen. The lateral side of the depressed area is the posterior end of the medial rim of the meatus ending in " $\mathrm{C}$ ". The quadrant-shaped depression described above may be called an ecto-tympanic cavity, being lateral to the primary and reptilian tympanic membrane. A septum, parallel with the lateral rim of the external auditory meatus, divides the roof of the ecto-tympanic cavity into an antero-lateral part, shallow and small, and a postero-medial part which is deep and wide. The septum is about $0.5 \mathrm{~mm}$. thick at its base (R.7088); I. $5 \mathrm{~mm}$. from its base, the thickness has decreased to about $0.2 \mathrm{~mm}$. and it can be surmised that it was considerably higher in the natural condition than it is in its present state. Approaching the narrow meatus proper, this septum loses height abruptly and merges into the roof of the meatus. In other words, the external auditory meatus widens medially into an ecto-tympanic cavity, which is divided into two compartments, both ending antero-medially in a high and thin wall. Before the most probable place of insertion of the quadrate and the quadrato-jugal is 
suggested, other pertinent features may be discussed: could the quadrate and quadrato-jugal of Oligokyphus occupy that position on the periotic, which the incus maintains in adult mammals? There are three answers to this question; $(a)$ the description of the periotic will reveal that nowhere on this bone-complex is there an area which could serve as an insertion for the quadrate or for the quadrate and the quadratojugal. (b) When the skull is dealt with as a whole, it will be evident that the quadrate cannot be placed farther medially than below the squamosal. (c) The osteology of Oligokyphus reveals very many reptilian and several very striking mammalian features; it seems very improbable that a composite lower jaw would go together with a quadrate which was already in the true mammalian position. In deciding the insertion area of quadrate and quadrato-jugal, some elements of the posterior part of the squamosal can be excluded in advance because of their observable or established function. The oval depression, anterior to the ecto-tympanic cavity, houses the lateral end of the paroccipital process (R.709o) and cannot therefore have held the quadrate or the quadrato-jugal. Medially of this depression is the superior wall of the post-temporal foramen. From this consideration it seems that there is no other possible place for the quadrate and the quadrato-jugal than the anterior end of the two compartments into which the ecto-tympanic cavity is divided. Corroborative evidence for this conclusion will appear when the skull is dealt with as a whole. Finally, mention should be made of a rounded and concave area anterior to the ecto-tympanic cavity, which suggests itself as the glenoid cavity of a secondary jaw articulation. It is significant that it is found here, anterior to the primary articulation of the lower jaw, and at the exact place where it is required by comparative anatomists in the mammalian ancestor. Clearly, however, the tip of the dentary of Oligokyphus did not come in contact with it. The quadrate intervenes between the posterior tip of the dentary and the roof of the ectotympanic cavity in a vertical direction.

IV. The zygomatic process.

Diademodon: the whole lateral surface of the zygomatic process is covered by the jugal. The anterior two-thirds of the zygomatic process are ensheathed by the jugal in such a way that only the upper surface of the zygomatic process is not covered by this bone.

Bienotherium : the specimen does not provide any evidence regarding the zygomatic process.

Oligokyphus : there is considerable variability in this area.

R.7088: the medial rim of the external auditory meatus is the inferior margin of the zygomatic process; along its whole length it is sharp and uninterrupted.

R.7098: the inferior margin of the external auditory meatus is smooth and rounded to within $5 \mathrm{~mm}$. of its anterior end ; anteriorly its condition is as in the previous specimen.

R.7094 and R.7096: the medial rim of the external auditory meatus is smooth and rounded with the exception of the anterior $7 \mathrm{~mm}$., where it is sharp, flattened and drawn out inferiorly, presumably for the insertion of a muscle. This sharp edge ends anteriorly in a distinct indentation and is preceded by a similar sharp edge which is straight and 
which encloses a plano-convex niche inferiorly and on the lateral side of the zygomatic process. This niche houses the posterior end of the jugal. Approaching the anterior end of this contact-surface, the inferior margin of the zygomatic process lies for a short distance (R.7094, R.7097) in a shallow cleft of the jugal, until the contact of the jugal on the lateral side of the zygomatic process ends and the one on the medial side enlarges. Finally the jugal covers the anterior end of this surface completely.

\section{THE BRAIN-CASE}

(Plate 5, fig. 3a, Plates 7-9; Text-figs. I3, I4)

Material: The principal specimen is R.7090 which consists of a right and left exoccipital, periotic, parietal, supra- and basioccipital, basisphenoid, left tabular and squamosal. The other specimens are :

R.7Iog right periotic.

R.7IIo left periotic, exoccipital and basioccipital.

R.7III , , , basioccipital.

R.7II2 right ,, and tabular.

R.7II3 right and left periotic, exoccipitals and basioccipital.

R.7II4 left periotic.

R.7II5 fragment of left periotic.

R.7II6 right periotic, unprepared.

R.7II7 fragment of right periotic.

R.7II8 posterior part of basisphenoid.

R.7242 anterior ,, , , , with basipterygoid processes.

This is the first description of a brain-case belonging to a mammal-like animal intermediate in age between the Lower Triassic Diademodon (Watson, I9I3; Brink, in preparation) and the Paleocene Ptilodus (Simpson, I937). Complete ossification of the periotic and greater width of the medulla and the cerebellum are the main structural advances of the brain-case of Oligokyphus when compared with Cynodonts. The height of the posterior part of the skull is immensely increased by the enormous parietal and lambdoidal crests and by the basisphenoid which points vertically downwards. The extraordinary height of the cranium is not correlated with an equally increased brain-capacity. In fact, the high crests of the dermal bones of the posterior part of the skull are entirely for muscle-insertion and so is the deep space between the foramen magnum and the overhanging lambdoidal crest. Incidentally, though the posterior part of the floor of the brain cavity is slightly below the level of the foramen magnum, its roof rises only very little above the upper margin of this foramen. The lateral lobe of the cerebellum is actually below this level.

\section{EXOCCIPITAL}

\section{(Text-fig. I3)}

The exoccipital is fused with the supraoccipital and is in sutural contact with the tabular, opisthotic and basioccipital. The bone is perforated by three small foramina situated behind the jugular foramen, the posterior serving probably for a vein, the anterior two transmitting the XIIth nerve. The jugular foramen extends between exoccipital and opis- 

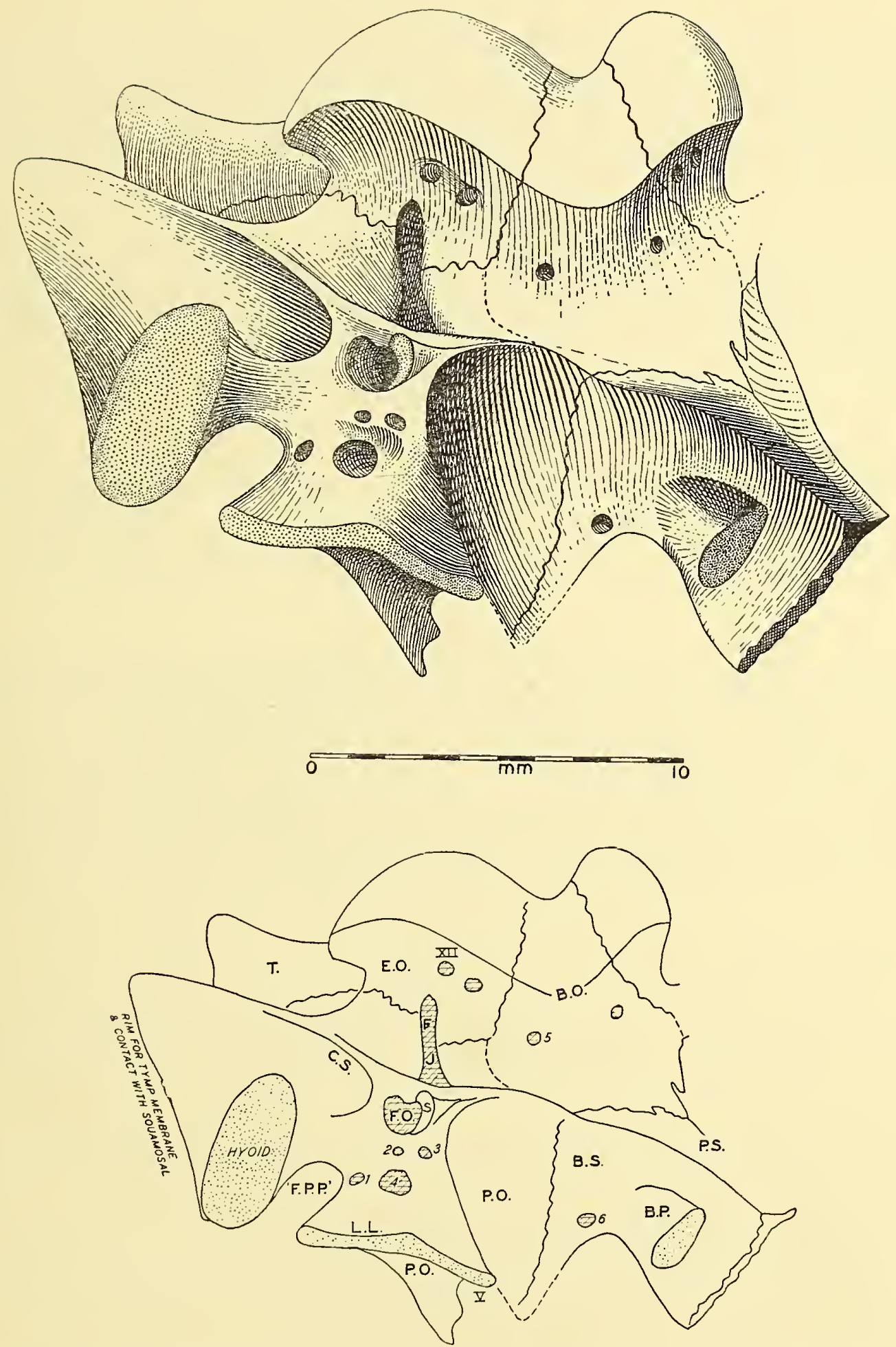

TEXT-FIG. I3.-The basicranium of Oligokyphus from below and laterally. Composite drawing, with key incorporating all available evidence. B.O., basioccipital ; B.P., basipterygoid process ; B.S., basisphenoid ; C.S., cavity for mus. stapedius ? ; E.O., exoccipital ; F.J., foramen jugulare ; F.O., fenestra ovalis ; F.P.P., notch, homologous with the foramen pterygo-paroccipitale of cynodonts; Hyoid., area of articulation of the hyoid arch on the anterior part of the paroccipital process ; L.L., lateral lamina of the prootic ; P.O., prootic ; P.S., parasphenoid, fused with basisphenoid ; $S$., contact area for stapes ; $T$., tabular ; I, foramen for superficial petrosal branch of VII ; 2 and 3 , foramina for VII ; 4 and 5, unidentified (vascular ?) foramina ; 6 , exit of VI ; V, Exit of V; XII, foramina for XII. 
thotic. The exoccipital forms the big stalked condyle and the margin of the foramen magnum above it.

The points of interest of the exoccipital are: the condyle reaches half way up the foramen magnum, whereas in Cynodonts it does not reach so high, and the condyle is larger than in Cynodonts.

\section{SUPRAOCCIPITAL}

This bone is fused with the exoccipitals and is in sutural contact with the tabulars. Though the evidence is not good, there seems to be a suture between the interparietal and the supraoccipital. Two small foramina observed on the outside of the supraoccipital are of no significance.

\section{BASIOCCIPITAL}

(Text-fig. I3)

The basioccipital is in sutural contact with the basisphenoid anteriorly. It is also in sutural contact with the exoccipitals. It forms the posterior part of the floor of the braincase. In the foramen magnum it forms the shallow notch between the condyles.

\section{PERIOTIC}

Opisthotic and prootic are fused in all the specimens and are described together. The periotic is in sutural contact with the exoccipital and with the basisphenoid. The tabular is fused to the opisthotic. The alisphenoid has a small sutural contact outside the cavum epipterycum with the anterior part of the "lateral lamina" of the prootic. The anterosuperior part of the periotic is covered by the parietal which provides the roof of the braincase in this position. Further back it is supposed that the parietals form the roof of the brain-case, until they meet the supraoccipital. Superiorly the periotic is fused with the parietal.

\section{Processes of the periotic}

These are the paroccipital process and, divided from it by the foramen pterygo-paroccipitale, the "lateral lamina" of the prootic. The former process is seen on all specimens, the latter only on R.7rog, though its posterior and most massive part is represented several times.

The paroccipital process of Oligokyphus is much shorter than in Cynodonts, and is equal to the distance from the sagittal plane to the lateral margin of the exoccipital condyle. In Cynodonts the whole lateral surface of the paroccipital process is in contact with the squamosal ; in Oligokyphus only the upper margin of the lateral face of the process has sutural contact with it. Its whole lateral face is excavated and forms the medial wall of the tympanic cavity. Its ventral surface too is concave and bears laterally an incision for the stapes. What has taken place through the stages which lead from a Cynodont condition to the one realized in Oligokyphus is a downward growth of the lateral end of the paroccipital process; this end has been deeply grooved for the stapes, the latter having remained in a horizontal, Cynodont-like position. The groove for the stapes divides the lateral end of the paroccipital process into a pointed posterior part and a rather massive anterior part. The latter has ventrally a wide perichondrial surface for the attachment of the hyoid arch. 
A feature already found incipiently in Diademodon is a conspicuous ridge, beginning faintly at the lateral end of the posterior part of the paroccipital process and increasing in height towards the fenestra ovalis. Anteriorly of this ridge is a distinct and elongated cavity, apparently to serve as a protective cover of some soft tissue, perhaps the stapedial muscle.

Anteriorly of the paroccipital process is the foramen pterygo-paroccipitale. In the strict sense this term is no longer applicable in Oligokyphus. The "foramen" is here a deep notch, open laterally because the short "quadrate ramus" of the alisphenoid is not long enough to close it. The anterior margin of this notch is formed by a horizontal lamina, which extends above the fenestra ovalis and below the exit of the fifth nerve, and is called the "lateral lamina" of the prootic. It constitutes the roof of the cavum epipterycum. The lateral lamina of the prootic of Oligokyphus has its structural homologue in Diademodon though here it is far less extensive. In Oligokyphus the lamina is perforated by four foramina whose function is obscure. The anterior margin of the lateral lamina was in contact with the alisphenoid.

\section{Foramina in the Periotic}

The foramen jugulare has already been mentioned; its anterior two-thirds are in the opisthotic.

The identity of the fenestra ovalis becomes evident on comparison with Cynodonts: it is situated medially of the foramen pterygo-paroccipitale and posteriorly of a lateral ridge on the opisthotic and basisphenoid which, approaching the sagittal plane, ends behind the basipterygoid process of the latter bone. The fenestra ovalis is almost vertical; the foot-plate of the stapes was in contact with only the lower half of the rim of the fenestra, where the bone shows by its slightly rough surface that it was covered by cartilage.

The deep plate, which in Diademodon separates the fenestra ovalis from the fenestra rotunda, is reduced in Oligokyphus to a rod of bone, I mm. in diameter. There is no common pit for the fenestra rotunda and the foramen jugulare as in Cynodonts and the openings of both are well below the fenestra ovalis. Immediately in front of the fenestra ovalis there are generally two foramina for the exit of the VIIth nerve; one, a small hole in the anterior wall of the foramen pterygo-paroccipitale, is a constant feature found in all specimens. The course of the canal of this foramen has not been followed. The position of the foramen in front of the fenestra ovalis indicates that it lodged the superficial petrosal branch of the facial nerve.

Above the anterior end of the lateral lamina of the prootic is the wide exit for the trigeminus.

Above the paroccipital process is a vascular canal, perforating the cranial wall and emerging in the subarcuate fossa. Outside the cranial wall this canal is joined by a groove from above, belonging to the vein of the sinus canal. Both structures leave the skull together through the post-temporal foramen. The course of the vein of the sinus canal is as follows : emerging from a canal presumably between the parietal and the periotic, it runs for a short distance in a groove horizontally backward and turns vertically downward above the canal from the subarcuate fossa. After the two have joined, they proceed again horizontally towards and through the post-temporal foramen. It is noteworthy that the canal which perforates the cranial wall from the floccular fossa to the exterior does not occur in Diademodon. 


\section{INTERNAL VIEW OF THE BRAIN-CASE}

(Text-fig. I4)

Compared with Diademodon the main differences of the internal wall of the brain-case of Oligokyphus are : the formation of a mammalian internal auditory meatus ; the distinct separation of three main structures, namely the jugular foramen, the internal auditory meatus, and the floccular fossa, due to complete ossification of the inner wall of the braincase and conditions in the floccular fossa, which are peculiar to Oligokyphus and have already been mentioned above.

The course of the sutures between exoccipital and opisthotic, as well as the position and direction of the jugular foramen, have been figured and do not require further elucidation. The two foramina for the hypoglossus are situated behind and below the jugular foramen.

Between the jugular foramen, the internal auditory meatus and the floccular fossa there is a small rough ridge lying between two elongated pits, whose features were at first obscure. There are three specimens which throw light on this difficult area: in R.7II5 a fracture terminates the specimen just in the middle of the ridge and exposes a section through the endocranial wall of the vestibule. It shows a canal, still filled with calcite, passing from the vestibule to the internal side of the brain-case. R.7II2, the only specimen which shows the tabular in perfect condition, is also fractured and allows a good view into the vestibule and the cochlea. Exactly opposite the rough ridge between the pits on the internal wall of the brain-case is a foramen in the vestibule. Finally R.7IIo shows the ridge between the two pits very well and reveals an elongated but very narrow foramen in it. The existence of a canal from the vestibule to the internal side of the brain-case and its position are therefore established beyond question. Comparison with Ornithorhynchus makes it certain that this is the cluctus endolymphaticus.

In front of the jugular foramen there lies a rather deep and round pit, whose base is perforated by three distinct foramina. The largest, posterior foramen opens into the proximal part of the cochlea and transmitted the cochlear branch of the acoustic nerve. The dorsal foramen opens into the vestibule and transmitted the vestibular branch of the acoustic nerve. The anterior foramen does not open into the vestibule nor into the cochlea and the course of the canal belonging to it has not yet been followed ; from its position this foramen must belong to the facial nerve. The Tritylodontidae evidently therefore possessed a perfect mammalian internal auditory meatus, and the approximation of the VIIIth and VIIth nerve, forshadowed in Diademodon, has gone much further.

Two millimetres anteriorly to the internal auditory meatus there is another pit, which has a rounded posterior margin. The bone, before it ends with a fracture in the middle of this pit, becomes very thin in all the available specimens and is notched, the notch being probably the posterior half of the exit of the Vth nerve, the pit having lodged the semilunar ganglion. Above the auditory meatus is the floccular fossa, which is of small diameter. The anterior vertical semicircular canal, surrounding the fossa, has been dissected in R.7II5. The fossa is continued laterally by a horizontal vascular canal already mentioned; there is also good evidence for a canal lying in a vertical position and connecting the vestibule with it.

Surrounding the flocculus above there is a distinct groove in the cranial wall which received the lateral lobe of the cerebellum. 

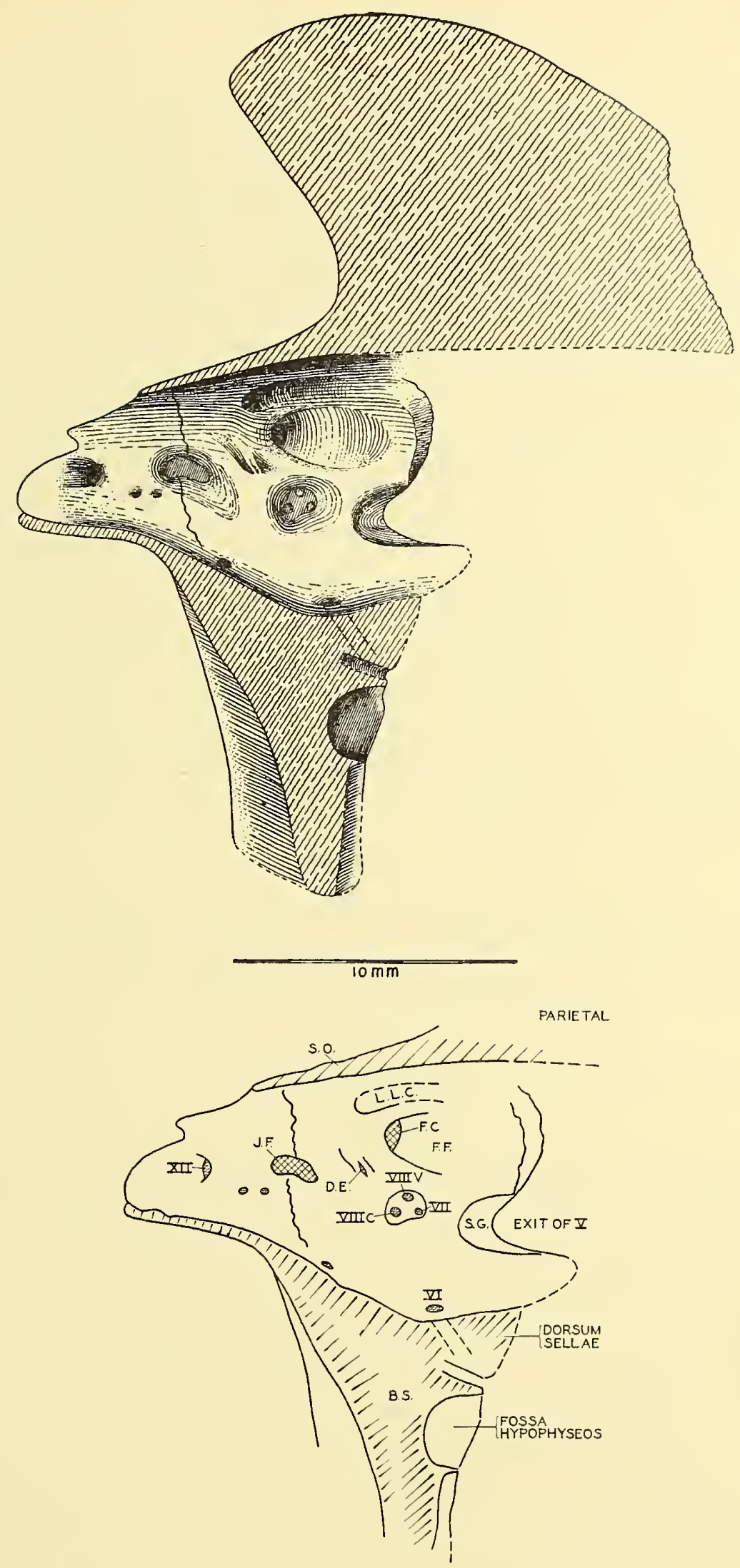

TEXT-FIG. I4.-The brain-case of Oligokyphus; inner aspect. Section in sagittal plane. B.S., basisphenoid; D.E., ductus endolymphaticus; F.C., floccular canal; F.F., floccular fossa; J.F., jugular foramen ; L.L.C., lateral lobe of cerebellum; S.G., site of semilunar ganglion ; S.O., supraoccipital in section ; $V I$, foramen for $\mathrm{N}$. abducens ; $V I I$, entry of facial nerve into internal auditory meatus ; VIII C, entry of cochlear branch of acoustic nerve into internal auditory meatus ; $V I I I V$, entry of vestibular branch of acoustic nerve into internal auditory meatus; $Y I I$, foramen for N. hypoglossus. 


\section{THE INNER EAR}

On R.709o the horizontal semicircular canal and part of the cochlea have been exposed. In R.7II6 the whole labyrinth and the cochlea, filled with matrix, have been freed from surrounding bone. A close fit has been achieved of the labyrinth and the two pieces of rock in which this inner ear is contained. Both specimens display a similar arrangement of cochlea and labyrinth. The vertical ridge in the periotic, beginning at the external side of the brain-case anteriorly of the fenestra ovalis and ending at the lowest point of the basisphenoid, houses the cochlea. The straight cochlea is about $5 \mathrm{~mm}$. long and about $\mathrm{I} .5 \mathrm{~mm}$. in diameter. The horizontal and the posterior semicircular canals each measure $3.5 \mathrm{~mm}$. The anterior semicircular canal is higher and the floccular canal passes through its loop. The crus commune rises from the posterior side of the labyrinth. Measured from the outside, at right angles to the sagittal plane, the angle between the horizontal semicircular canal and the cochlea is II $5^{\circ}$. The cochlea is vertical in R.7090 and the horizontal semicircular canal is inclined towards the sagittal plane at $25^{\circ}$. There is no noticeable inclination of the horizontal semicircular canal either towards the front or the back.

The vertical position of the cochlea is apparently a consequence of the almost vertical position to the (formerly horizontal) antero-basal part of the periotic and the basisphenoid. The cochlea has taken part in the downward movement of these two bones; it has not maintained its original horizontal position while the surrounding bone became vertical. The position of the cochlea in Nythosaurus (Simpson 1933) and in the Monotremes (Denker I90I) is more nearly horizontal.

\section{THE STAPES}

The stapes has not been found. The minimal length of this bone is the distance between the fenestra ovalis and the lateral end of the paroccipital process to which, as we have argued, the tympanic membrane was attached. This distance is $6 \mathrm{~mm}$. on both R.70go and R.7II4.

\section{THE BASISPHENOID}

(Plate 5, fig. $3^{a}$ )

Specimens R.7090, R.7III and R.7242 provide the evidence of this bone. Sutures are visible between it and the opisthotic on the ventral side as far forward as the fenestra ovalis. The bone is fused with the prootic and there is a faint suture with the basioccipital.

The basisphenoid forms the floor of the brain-case from its line of fusion with the basioccipital to the pituitary fossa. It is badly preserved in the great associated complex (R.7090) which is none the less invaluable in revealing the extraordinary length and position of the bone. The surface facing posteriorly corresponds with the ventral surface of the basisphenoid in earlier Therapsids and consequently the anterior surface is homologous with the dorsal surface of the bone in Cynodonts.

This aberrant development in Oligokyphus is correlated with the lowering of the facial axis in relation to the cranial axis, with the elevation of the jaw articulation high above the row of cheek teeth, and with the consequent lengthening of the masticatory muscles. Bienotherium is in this respect intermediate between Diademodon and Oligokyphus, and in the former there is only a slight tendency to lower the anterior end of the basisphenoid. Strictly 
carnivorous Therapsids of similar age to Diademodon do not show this pulling down of the basisphenoid nor is this development found in primitive Mammalia.

Specimen R.7III consists only of the most posterior part of the basisphenoid, nearest to the basioccipital. R.7242 is, however, most revealing and is anatomically perfect as far as it goes, though only very little is preserved of the cultriform process of the parasphenoid below and between the basipterygoid processes. The dorsum sellae, as well as the part of the anterior floor of the brain-case which is formed by the basisphenoid, are missing. The identification of this specimen is, however, certain ; it rests on the shape of the posterior surface, which is shown here and on R.70go, and on the occurrence of a canal leading from the anterior surface of the bone above and lateral to the fossa hypophyseos to the anterior part of the floor of the brain-case, near the mid-line. Through a break in R.7ogo this canal is open along its whole length and can be seen on Plate 8, fig. 3. The canal may have transmitted the VIth nerve or a vessel.

The posterior surface of the basisphenoid is drawn out laterally into a sharp and wide edge, as already mentioned in connexion with the periotic. The edge is continued upward to near the fenestra ovalis and contains the cochlea in its dorsal part.

The posterior surface of the basisphenoid-homologous with the ventral surface of this bone in earlier Therapsids-has no foramina ; it is thus clear that no carotid foramina exist in Oligokyphus.

The anterior surface of the basisphenoid has two stout basipterygoid processes, for the articulation with the epipterygoids, which face downward and very slightly forward. On the same level as the articulating surfaces of these basipterygoid processes and between them is found the floor of an oval cavity, which goes deep into the basisphenoid. This cavity must be the fossa hypophyseos; its margins are sharp except for the upper quarter of its lateral walls, and those smooth and rounded areas are regarded as providing for the entry of the internal carotids into the fossa hypophyseos. The course of the internal carotid in this region was, therefore, as follows : vertically upward between the basisphenoid and the epipterygoid, immediately posterior to the basipterygoid process, and then passing behind the basipterygoid process dorsally and bending anteriorly and medially into the fossa hypophyseos near its roof. In Young's text-fig. 3 (I947) the actual course of the internal carotid is about $5 \mathrm{~mm}$. medially of the end of the bar labelled "I. Car".

Above the fossa hypophyseos there is a small medial foramen; its canal faces backward but its course has not been traced. On each side of this foramen is another, described above, on R.7090 as the probable exit of the VIth nerve.

THE BRAIN OF OLIGOKYPHUS

(Plate 8, figs. 2, 3 ; Text-figs. I4, I5)

The only satisfactory evidence is provided by the natural cast of the brain-cavity on R.7090, where the anteror part of the medulla oblongata has been freed entirely from the bone and the right side of the brain-cavity is exposed up to the exit of the N. trigeminus.

The surface of the brain-cavity is smooth. Anterior to the exit of the $\mathrm{N}$. trigeminus the transverse diameter of the brain-cavity decreases slightly. The sagittal plane of the cavity proper is not exposed in this cast. As the cast does not reach beyond the exit of the trigeminus all of the brain-cavity available must belong to the cerebellum. No evidence of 
the mid-brain and the fore-brain is provided apart from their estimated length. According to the reconstruction of the whole skull, the brain has a length of $40 \mathrm{~mm}$., measured from the bottom of the deep notch in the supraoccipital to the suture of the mesethmoid with the frontal, which is situated below the bifurcation of the sagittal crest. The cerebellum takes almost one half of the length of the brain, being at least $18 \mathrm{~mm}$. long, the olfactory lobes have a minimum length of $8.5 \mathrm{~mm}$. and mid- and fore-brain together measure $13.5 \mathrm{~mm}$. or less.

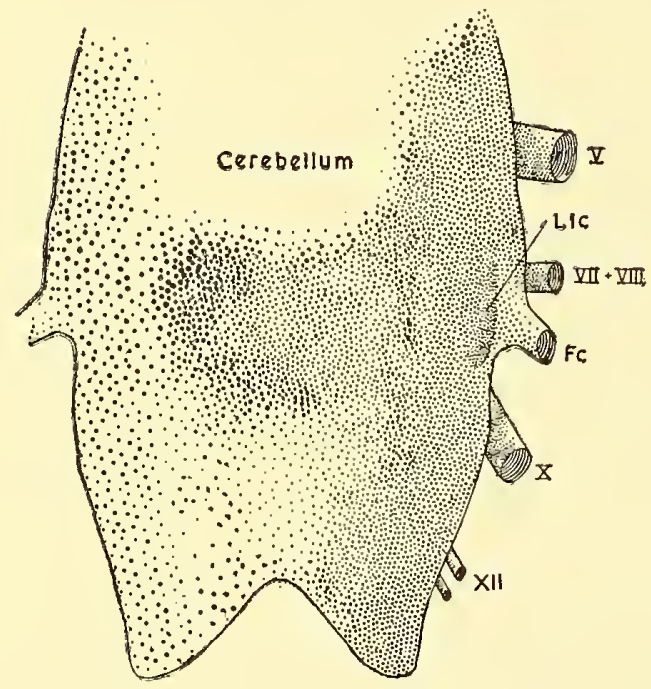

FIG. I5A.
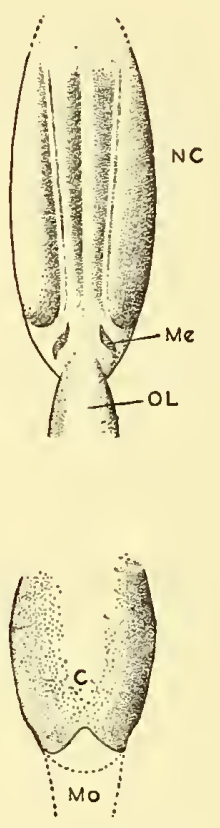

FIG. I5B.

TEXT-FIG. I5A.-Cast of brain cavity of Oligokyphus. The cerebellum. Fc, flocculus ; Llc, impression of lateral lobe of cerebellum; $V, V I I, V I I I, X, X I I$, cranial nerves. Twice nat. size.

TEXT-FIG. I5B.-Reconstruction of brain. $C$, cerebellum; $M e$, mesethmoid; $M o$, medulla oblongata; $N C$, nasal cavity; $O L$, olfactory lobes. Nat. size.

The cerebellum has its maximum width of $\mathrm{I}_{4} \mathrm{~mm}$. at the level of the exit of the $\mathrm{N}$. trigeminus and its maximum height of about $9 \mathrm{~mm}$. at the level of the exit of the (?) VIth nerve.

The fossa hypophyseos is normal for an animal of this size, but its position deep below the floor of the cerebellum is extraordinary and aberrant. The sella turcica, forming an acute angle, is enormous and recalls a condition found in Sauropods. The excessive growth and the turning down of the basisphenoid have affected the position of the fossa ; though anatomically still on the dorsal surface of the basisphenoid it is yet topographically on its anterior surface.

The cerebellum merges imperceptibly into the medulla oblongata.

Though the cerebellum of Oligokyphus is larger than that of Cynodonts there is as yet no indication that mid- and fore-brain were advanced beyond a Cynodont condition. The brain of Oligokyphus is essentially of Cynodont pattern, and all known mammalian brains are distinguished from it by the preponderance of their neopallium. 


\section{THE EPIPTERYGOID}

(Plate 5, fig. $3 b$, Plate Io, fig. I $a, b$; Text-fig. I6)

The identification of the epipterygoid rests on twofold evidence: $(a)$ the basipterygoid process of R.7242 is matched exactly by a contact area of the same character in R.7237 (two cartilage bones meeting with smooth, not serrated, surfaces devoid of periosteal bone) ; (b) after the identification of the skull bones is nearly completed, the unidentified bones and unfilled spaces in the skull are few. To identify the bones in question as the epipterygoid raises no inconsistency if it is assumed that the bone is essentially similar in Oligokyphus and in Cynodonts.

The ventral margin of the epipterygoid is straight and parallel with the sagittal plane from the anterior end of the bone to its articulation with the basisphenoid. Behind this articulation the lower margin diverges from the mid-line. Thus, seen from outside, the epipterygoid

B

A
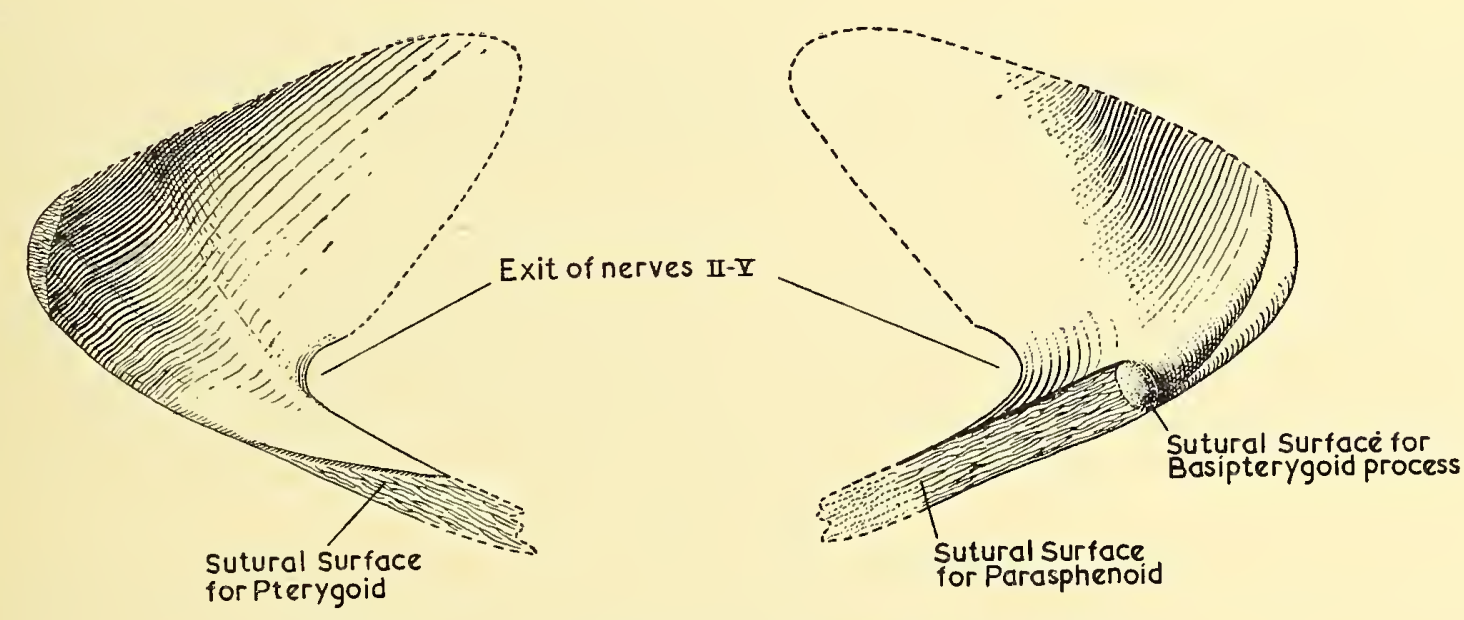

TEXT-FIG. I6.-Epipterygoid of Oligokyphus. $\times 2 \cdot 5$.

is concave (R.7236, Plate Io, fig. Ib). If the lower margin of the bone is placed horizontally the articulating surface with the basipterygoid points directly backward and very little upward. However, as the articulating surface of the basipterygoid process actually points almost vertically downwards the orientation of the epipterygoid must be in accordance. This means that the posterior part of the bone (which in Cynodonts is the quadrate ramus) is on a higher level than the anterior part and even more so than in R.7237 (Plate Io, fig. I $a$ ).

All three illustrated specimens have the posterior end well preserved: it is slightly thickened and irregular (not smooth). This is exactly as in Watson's (I920: 5I9) description of this part of the epipterygoid in Protacmon. The contact of the superior end of the epipterygoid with the lateral lamina of the prootic was probably ligamentous. The exit of the trigeminus from the periotic is situated medially to the lateral lamina of the prootic (Plate 7, fig. 4), thus the whole width of the lateral lamina intervenes between the posterosuperior margin of the epipterygoid and the exit of the trigeminus, and it is impossible that the straight ascending ramus of it could have formed the anterior part of this exit, as is so well shown by Parrington (I946, fig. 7a). The spot where the mandibular branch of the 
trigeminus passed over the superior margin of the epipterygoid was approximately where each of the two bones (Plate Io, fig. I) has a small fragment missing from this margin. Certainly the epipterygoid was not notched for the trigeminus. It is doubtful if the ascending ramus of the epipterygoid touched the parietal dorsally, though this process was certainly longer than in the only specimen which shows it (R. 7239, Plate 5, fig. $\left.3^{b}\right)$; its upper point might have ended freely without bony contact of any sort.

Only the lowest part of the anterior margin of the ascending process, where it is notched for the exit of nerves II to VI, is preserved (R.7236, Plate Io, fig. Ib). Anteriorly there is a rather narrow process, which is overlapped by the pterygoid.

The lower margin of the epipterygoid has a medial sutural surface from its anterior end to the articulation with the basipterygoid process. This surface must have been in contact with the cultriform process of the parasphenoid, which is not available.

\section{THE PTERYGOID}

(Plate Io, fig. 2c)

By comparison with Diademodon and Bienotherium five specimens (R.7243-47) can be identified as the flanges of this bone. These fragments are triangular, having on their lateral side a straight surface, with a characteristic relief, that was tightly applied to the "boss" on the dentary.

The point of the pterygoid flange in Cynodonts is immediately behind and considerably below the tooth row, if the jaw is closed. Owing to the backward extension of the tooth row in Oligokyphus the point of the pterygoid flange does not pass below the tooth row, but comes to rest on this level, between the "boss" and the alveolar border. The pterygoid's share in the palate is uncertain, and no specimen throws light on this area.

\section{THE VOMER}

The vomer is not represented, nor is any bone which is in sutural contact with it. The dorsal side of the maxillary above the sagittal suture ought to bear an indication of a vomerine contact, but this is lacking, even on the good anterior fragment R.7209 and on R.7027. The ventral edge of the vomer must have been inserted into the sagittal suture between the maxillaries.

\section{THE PALATINE}

(Plate Io, figs. $2 a, b, 3 a, b, 5$ )

Specimens numbered R.7248 and R.7249 can be identified as right palatines with the help of R.7206 which consists of the posterior part of the right maxillary, the anterior part of the jugal, very little of the lacrimal with the entry of the suborbital canal and a small part of the palatine. The crucial part of this fragment of palatine is not the medial part, applied to the maxillary - and only available in this specimen-but a gutter leading backwards from the posterior end of the entry of the suborbital canal and ending, with a fracture, as a canal which points backwards and down. In both isolated palatines this canal and the gutter are well preserved, and the canal is seen to emerge on the ventral side of the bone, immediately behind a spacious cavity, which houses the posterior end of the maxillary. If it be assumed that this ventral opening of the canal in the palatine is for the greater 
palatine nerve-assuming that the name given for the pertinent foramen in Cynodont skulls implies such an identification-it follows that this nerve runs backward from the entry of the suborbital canal and underneath the maxillary branch of the trigeminus. It is likely that the wide entry of the suborbital canal may have lodged the spheno-palatine ganglion.

The difficulties encountered in the identification of the palatine are mainly because no isolated and complete Cynodont palatine is available. The most puzzling feature of this bone in Oligokyphus is an elongated dorsal pocket, widening anteriorly, where its high medial rim abuts on the lateral margin of the entry of the suborbital canal. Anterior to this spot no part of the palatine is preserved, but the numerous maxillaries showing the sutures with this bone prove that a considerable part is missing, that it was below the level of the two isolated fragments available, and that taking into account the condition in Diademodon and Bienotherium, the palatine has a large share in the secondary palate.

DERMAL BONES OF THE BRAIN-CASE AND THE ROOF OF THE SKULL

Interparietal

No sutures are visible between this bone and the tabular and the supraoccipital. As it is unlikely that the latter bone reached the parietal crest and actually covered the posterior and medial surface of this structure, the presence of the interparietal is indicated.

\section{Tabular}

The tabular is distinct in R.709o, where its contacts with the squamosal and the parietal can be studied. Good sutures are developed between it and the supraoccipital and the exoccipital. The upper half of the posterior margin of the post-temporal foramen is formed by the tabular. R.7IIo shows the fusion of tabular and opisthotic.

\section{Parietal}

Sutures:

(a) With the interparietal on the medial part of the lambdoid crest.

(b) With the tabular for a short distance on the lambdoid crest.

(c) With the medial margin of the squamosal along a straight suture.

(d) With the frontal on a deeply serrated suture. (Evidence provided by the frontal.)

(e) The parietal is fused with the opisthotic. In the anterior margin of the prootic there is a deep slit which housed the parietal. (R.7IIo, Plate 9, fig. 2.)

$(f)$ With the parietal of the opposite side on a wide sutural surface in the sagittal plane; both parietals are always co-ossified.

The principal specimen is R.709o and there are only three other specimens incorporating the posterior part of the parietal crest. The salient features of the parietals are the enormous sagittal crest and the similarly extended lambdoid crest, the latter sloping backward, overhanging the supraoccipital. Since the posterior part of the frontal has a steep lateral slope, the medial crest evidently extended to the posterior part of this bone. The anterior third of the parietal is not available, and thus it is impossible to say whether this Tritylodontid at least possessed a parietal foramen. Bienotherium also lacks the pertinent part 
of the parietal and Young (I947:548) is wrong in assuming that there is evidence for the absence of the parietal foramen in the type skull of Tritylodon longaevus. No parietals are preserved in this specimen.

Nothing is known about the roof of the brain-case where this is formed by the parietals.

The Frontal (Plate Io, figs. 3c, 4)

The collection is unexpectedly rich in frontals ; there are sixteen registered specimens, R.72I3-7228, and ten others. R.72I3 and R.72I4 have both frontals co-ossified.

Sutures :

(a) With the parietal as discussed.

(b) With the nasal along a deeply serrated suture.

(c) With the upper end of the facial part of the lacrimal.

(d) The frontal must have been in contact with the orbitosphenoid though no evidence is available.

(e) The frontal has a sutural surface with the mesethmoid when the latter becomes ossified in old individuals (R.72I5, Pl. Io, fig. 3c).

But for its contact with the nasal and with the frontal of the opposite side, the frontal is overlapped by all other bones with which it is in contact.

The sagittal crest, disappearing as such on the anterior half of the frontals, is continued by two bifurcating edges, each passing to the antero-lateral end of the frontal and forming there a supraorbital prominence. The upper surface of the two bones between the bifurcating edges is rugose and flat.

The inner surface of the frontals is in all respects similar to that of Diademodon as shown by Watson (I9I3:223), but for the absence of separate impressions of the olfactory lobes in Oligokyphus. Anteriorly of the suture with the mesethmoid (R.72I5), there is a medial ridge formed by both frontals and supporting the vertical lamina of the mesethmoid. Laterally to the suture, and parallel to the sagittal plane, there is a double ridge, supporting ethmoturbinals. Both ridges are continued on the nasals.

\section{The Nasals}

The seven specimens of nasal, R.7229-7235, are poorly preserved. The anterior end is missing in all cases.

Sutures :

(a) With the frontal as already discussed.

(b) With the maxillary along its lateral margin. The maxillary overlaps the nasal and the suture is serrated (R.7229, R.7232).

(c) With the septomaxillary ) At the antero-lateral point of the nasal. Evidence

(d) With the premaxillary $\}$ from premaxillary.

The nasal is a flat, thin, long bone; apart from the ridges on its underside already mentioned, there is nothing which requires description. The width, the only available dimension, is from 6.4 to $8.0 \mathrm{~mm}$. 
Though Cynodont skulls are well known and available in many collections, their more detailed osteology has still to be written. Of many bones only the outer aspect is known, and some of the difficulties of the present work arise because in Oligokyphus skull bones have been preserved in their full intricacy. Furthermore, it was unexpectedly difficult to apply a knowledge of Cynodont skull material to some of the skull bones of Oligokyphus. Trends which became obvious after identification was made had been unnoticed before; for instance, the extension of the basisphenoid downwards was never imagined before the specimen R.709o was developed; the two isolated palatines lacked identification for years until redevelopment of the complex R.7206 revealed one corresponding character.

The account of the palatal bones is admittedly inadequate, but the meagre evidence does not allow more to be said and though it appears that the ectopterygoid is lost in Oligokyphus, there is no objective evidence to prove the assumption.

\section{RECONSTRUCTION OF THE SKULL}

(Text-figs. I7-20)

The attempted reconstruction of the skull, based with great care on the specimens described above, may be in some points conjectural but gives, on the whole, an adequately accurate picture.

The orientation of the skull is based on the assumption that a plane through the apices of the cusps of the upper cheek teeth is horizontal. The orientation of the maxillary is obvious as the sagittal suture is preserved in many specimens. The premaxillary suture is preserved on R.7027 and gives the distance of the premaxillary from the first cheek tooth. The palatine, with its ventral concavity, fits the posterior end of the maxillary and is thus brought into place ; moreover there is also R.7026 with part of the palatine in association. The same specimen has the distal part of the jugal in association and is essential for the orientation of this bone. The position of the pterygoid flanges is determinable by their juxtaposition against the thickened "boss" on the dentary above the last cheek teeth. Both are in contact and help to maintain the position of the jaw during mastication. With this complex of bones one can associate the dentary, by bringing the upper and lower cheek tooth dentition into occlusion. The posterior end of the dentary determines the position of the articular notch in the articular, and into this notch fits the quadrate. The trochlea and the dorsal peg of the quadrate determine the position of the squamosal. The distance of the quadrate from the sagittal plane can be checked by the distance of the quadrate articulation on the squamosal in R.7090. This specimen also gives the total length, but no other characters, of the basisphenoid ; R.7242, however, uncrushed and similarly orientated, gives with its basipterygoid processes the articulation of the epipterygoids.

The dermal bones of the skull roof have produced difficulties, as the contact between frontal and parietal is not available. The frontal is therefore arranged as in Bienotherium; here its prominent supraorbital boss is continued downward by a ridge on the lacrimal which separates on this bone a facial and an intraorbital part. Fronto-nasal sutures are available, but the anterior extension of the nasals is conjectural.

The greatest gaps in knowledge of the skull are apparent in the lateral view. The actual curvature of the zygomatic arch may be a little different and evidence is lacking of the large 


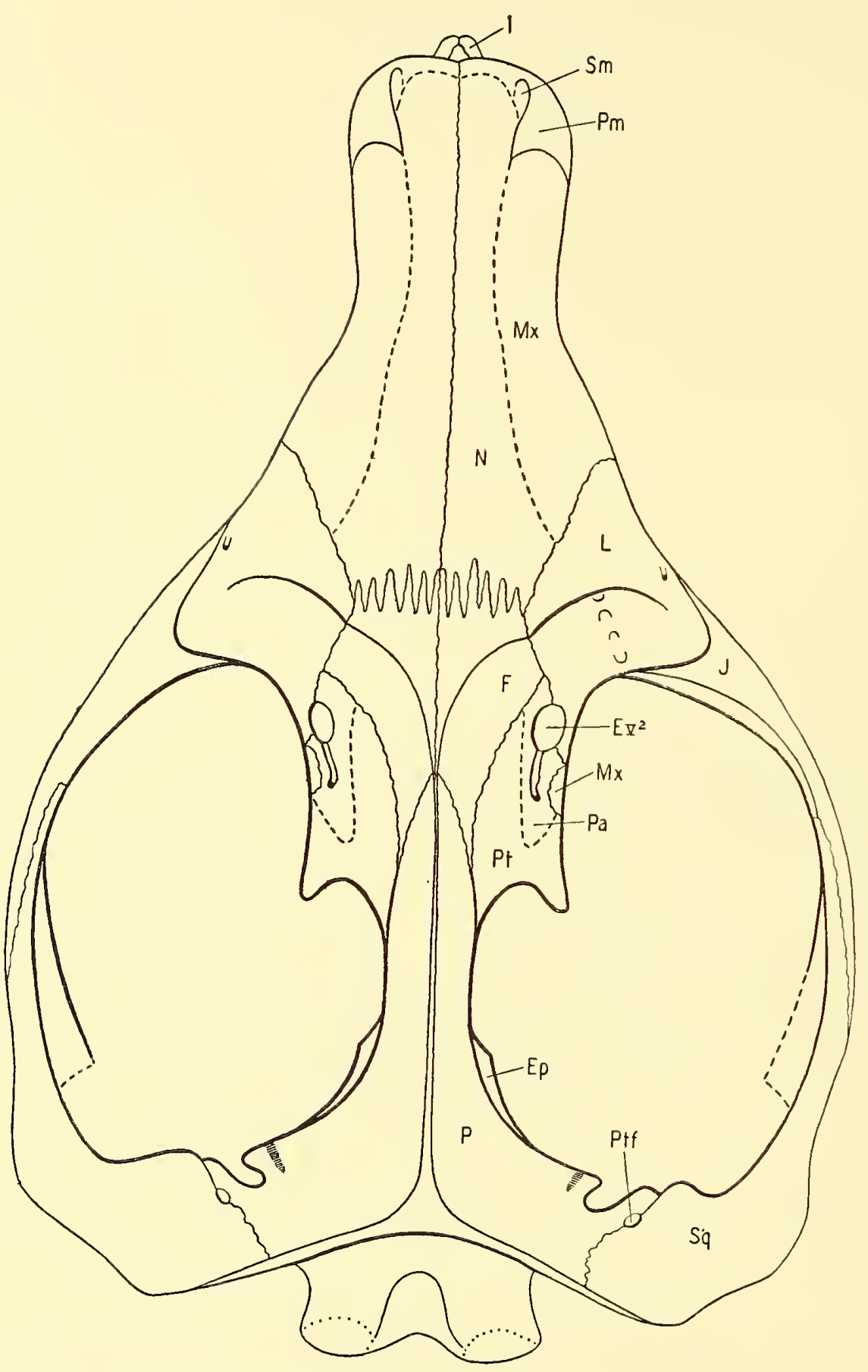

TEXT-FIG. I7.-Reconstruction of the skull of Oligokyphus. Dorsal view. $\times 2$. Abbreviations used in this and the following five text-figures: $A$, articular ; $B o$, basioccipital ; $B s$, basisphenoid ; $E c$, ectopterygoid ; $E$ o, exoccipital ; $E p$, epipterygoid ; $E V^{2}$, entry of fifth nerve into lacrimal ; $F$, frontal ; $F m$, foramen magnum ; $F o$, fenestra ovalis ; $F p$, foramen-pterygo-paroccipitalis ; $H p$, articulation of hyoid on paroccipital process ; $I$, first incisor ; $I p$, interparietal ; $J$, jugal ; $J f$, jugular foramen ; $L$, lacrimal ; $L l$, lateral lamina of prootic ; $M x$, maxilla ; $N$, nasal ; $P$, parietal ; $P a$, palatine ; $P m$, premaxillary ; $P o$, postorbital ; $P p$, paroccipital process ; $P s$, parasphenoid ; $P t$, pterygoid ; Ptf, post-temporal foramen; $Q$, quadrate; $Q j$, quadrato-jugal ; $S m$, septomaxillary ; So, supraoccipital; $S q$, squamosal ; $T$, temporal ; $V$, vomer. 


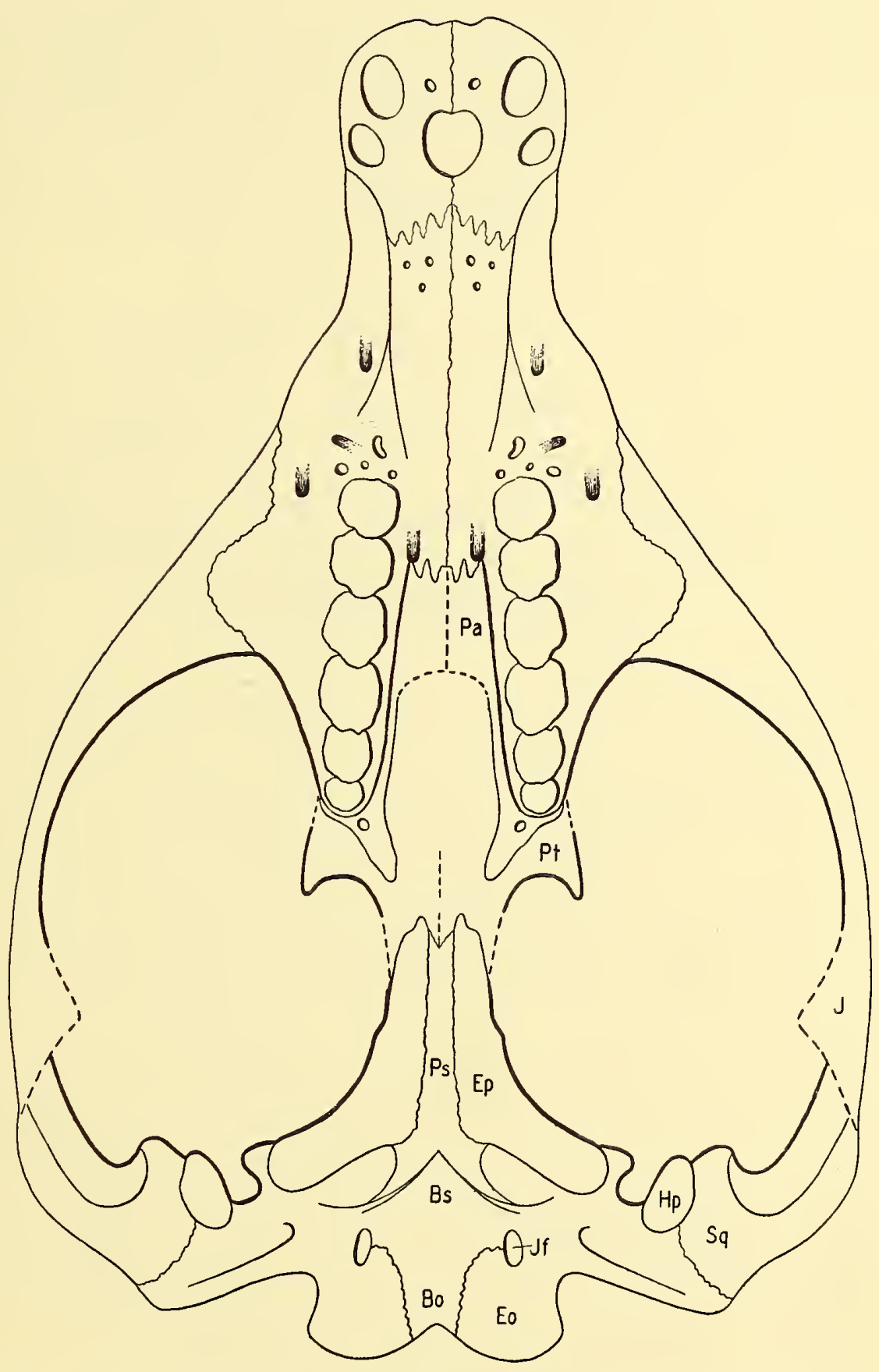

TexT-Fig. I8.-Reconstruction of the skull of Oligokyphus. Palatal view. $\quad \times 2$. Lettering as for Text-fig. I7. 
region below the frontal and behind the lacrimal. It is suggested that there was an orbitosphenoid.

Late Cynodonts have a triangular, flat and box-like skull with wide zygomatic arches of enormous strength. The major jaw muscles are thick and short, and the masseter equals the temporalis. The zygomatic arch is an essential structure of the skull, giving it stability during the capture of active prey. In dorsal view the skull of Oligokyphus is bottle-shaped:

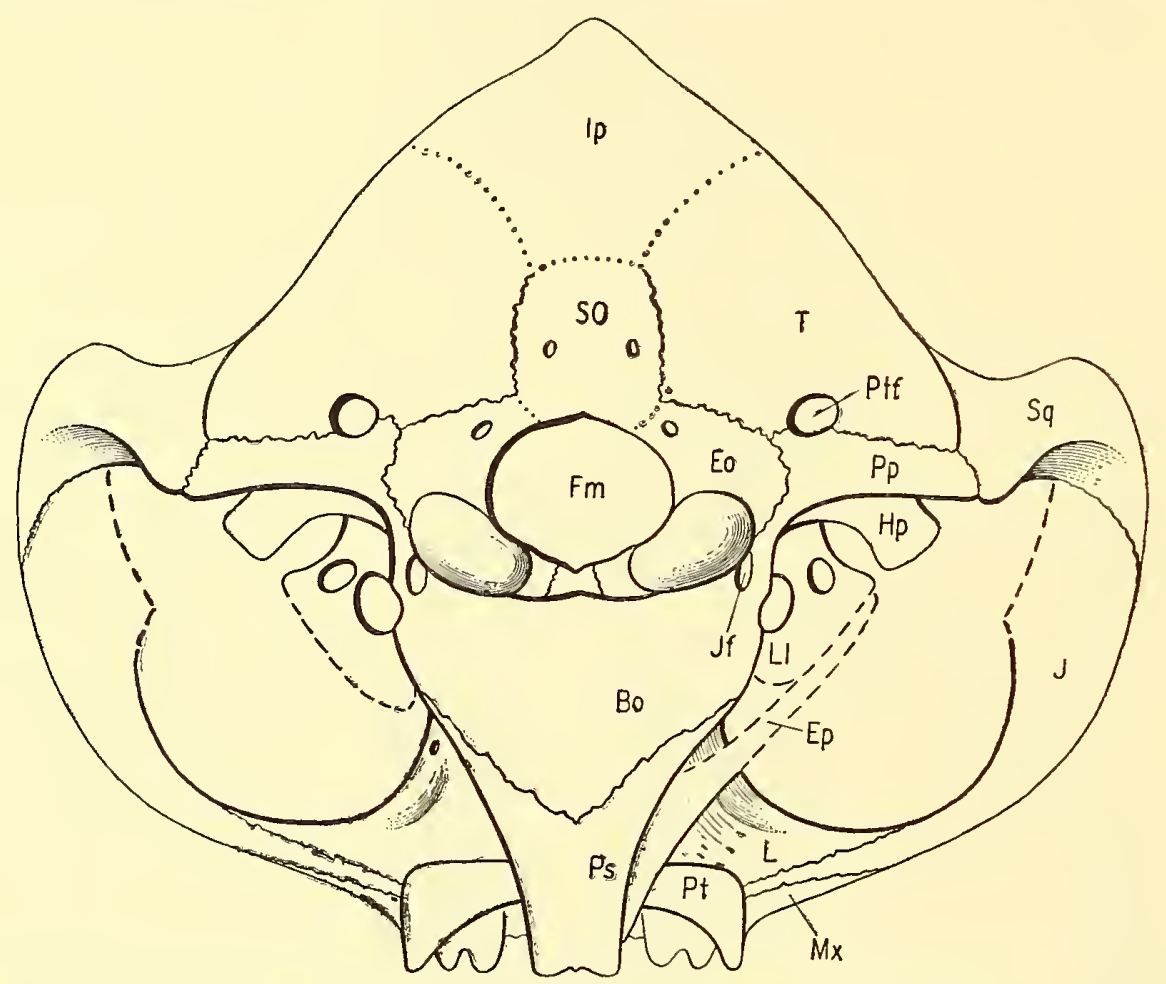

TEXT-FIG. I9.--Reconstruction of the skull of Oligokyphus. Posterior view. $\quad \times 2$. Lettering as for Text-fig. I 7 .

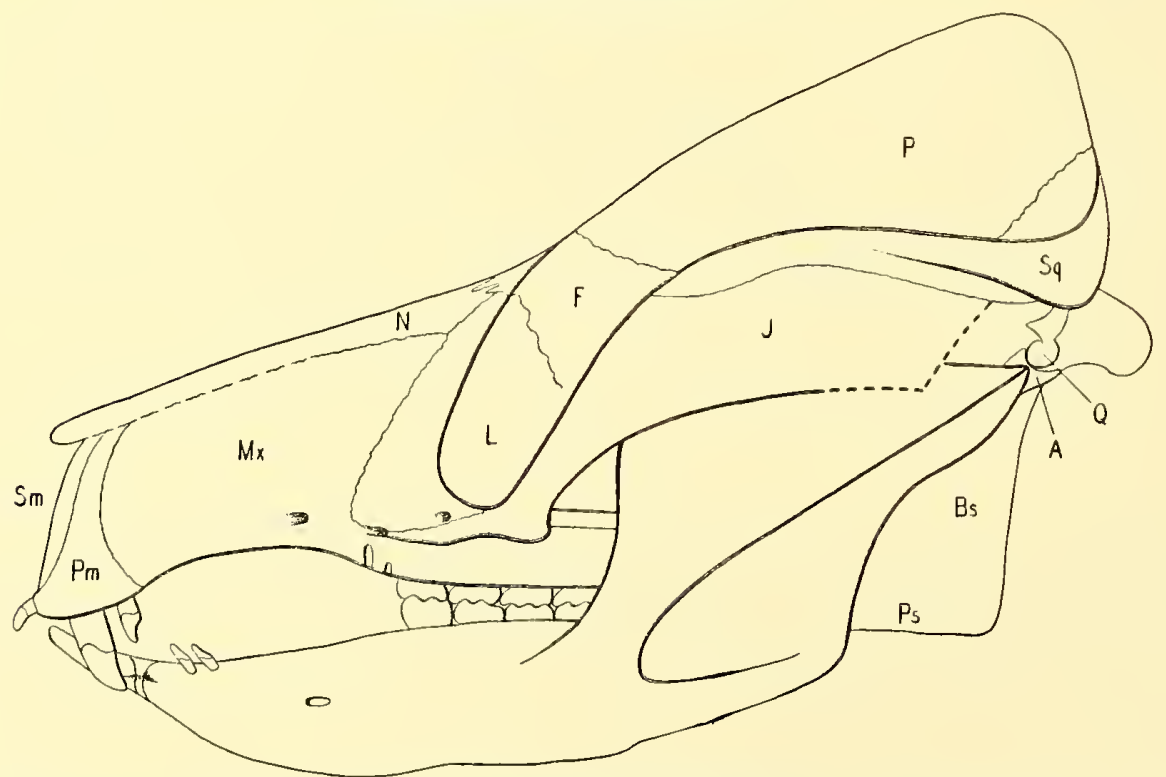

TEXT-FIG. 20.-Reconstruction of the skull of Oligokyphus. Lateral view. $\quad \times \mathrm{I}^{\circ} 5$ Lettering as for Text-fig. I 7 . 
its zygomatic arch is rather weak and there is a high occipital crest. Consequently the temporalis is much stronger than the masseter and the function of the zygomatic arch is negligible. The strong sagittal crest compensates this weak zygomatic arch and, apart from that, the herbivorous Oligokyphus can afford to have a skull built more lightly than a carnivorous Cynodont. The herbivorous habit of Oligokyphus is also borne out by the dentition (a battery of identical cheek teeth adapted for cutting fibres), by the diastema, by the articulation of the lower jaw high above the tooth row and by the posterior cheek teeth being inside the coronoid process in the temporal fossa.

A comparison of the skulls of Oligokyphus, Diademodon and Thrinaxodon reveals the following points :

I. The secondary palate increases posteriorly from Thrinaxodon to Oligokyphus; in the latter it reaches the middle of the skull.

2. The hinder end of the cheek tooth series extends more and more posteriorly from Thrinaxodon to Diademodon and to Oligokyphus.

3. The temporal fossa devoid of a postorbital bar in Oligokyphus extends unusually far forward. The jaw articulation of Oligokyphus being at the posterior end of the skull, as in Cynodonts, masseter and temporalis are hence in a more effective position. Their pull acts at right angles on the dentary and the anterior limit of the insertion area of the masticatory muscles is now in front of the posterior cheek teeth.

These same features can be found in the much smaller and less aberrant Ictidosaurs A and B of Broom (I929, I932) and hence are indicative of relationship. Also shared by these two specimens and the Tritylodontids is the elevation of the basis cranii above the palate. This feature is unknown in Cynodonts, Mesozoic mammalia and Monotremes. The enigmatic Kunminia does not display it, nor does it show the backward extension of the row of cheek teeth into the temporal fossa.

The elevation of the basis cranii above the palate reaches its maximum in Oligokyphus; here it is correlated with an herbivorous diet. In other groups of Therapsids and mammalia, herbivorous diet is correlated with an oblique skull posture or a skull architecture not found in carnivores. In Dicynodonts, for instance, the skull is inclined forward, the occiput being vertical and the cranial axis sloping. Artiodactyls have a distinct angle between the basicranial and the facial axes. In Tritylodontids both the basicranial and the facial axes are parallel and horizontal but the latter lies deeply below the level of the former. Elephants, Manatus, and Nototherium recall the condition found in Oligokyphus, but in these mammals the basisphenoid is never concerned in this deviation from an insectivorous or carnivorous condition. The behaviour of this bone in Tritylodontids is unique. The vertical basisphenoid of Oligokyphus separates the Tritylodontidae from any known mammal.

\section{THE MIDDLE EAR OF OLIGOKYPHUS}

The evidence bearing on the middle ear and the jaw-articulation of Oligokyphus has been presented earlier and only a summary need now be given. Ample evidence is available for the dentary, squamosal, periotic, quadrate and the articular complex. The dentary articulates by means of the articular complex only and no contact with the squamosal is made. The antero-posterior movement of the jaw is possible on account of the loose articulation of the quadrate with the squamosal. A long anteriorly directed external auditory meatus is present, ending posteriorly at the region of the quadrate-squamosal articulation. 
The articular complex bears on its medial side a muscle insertion for part of the pterygoideus internus which is a homologue of the tensor tympani. A rim on the distal end of the paroccipital process indicates the position of the tympanic membrane. The long stapes, which has not been found, must have had a horizontal position.

The following features of Oligokyphus show an approach to a mammalian condition beyond a stage reached in Cynodonts: The coronoid is lost; the dentary is relatively

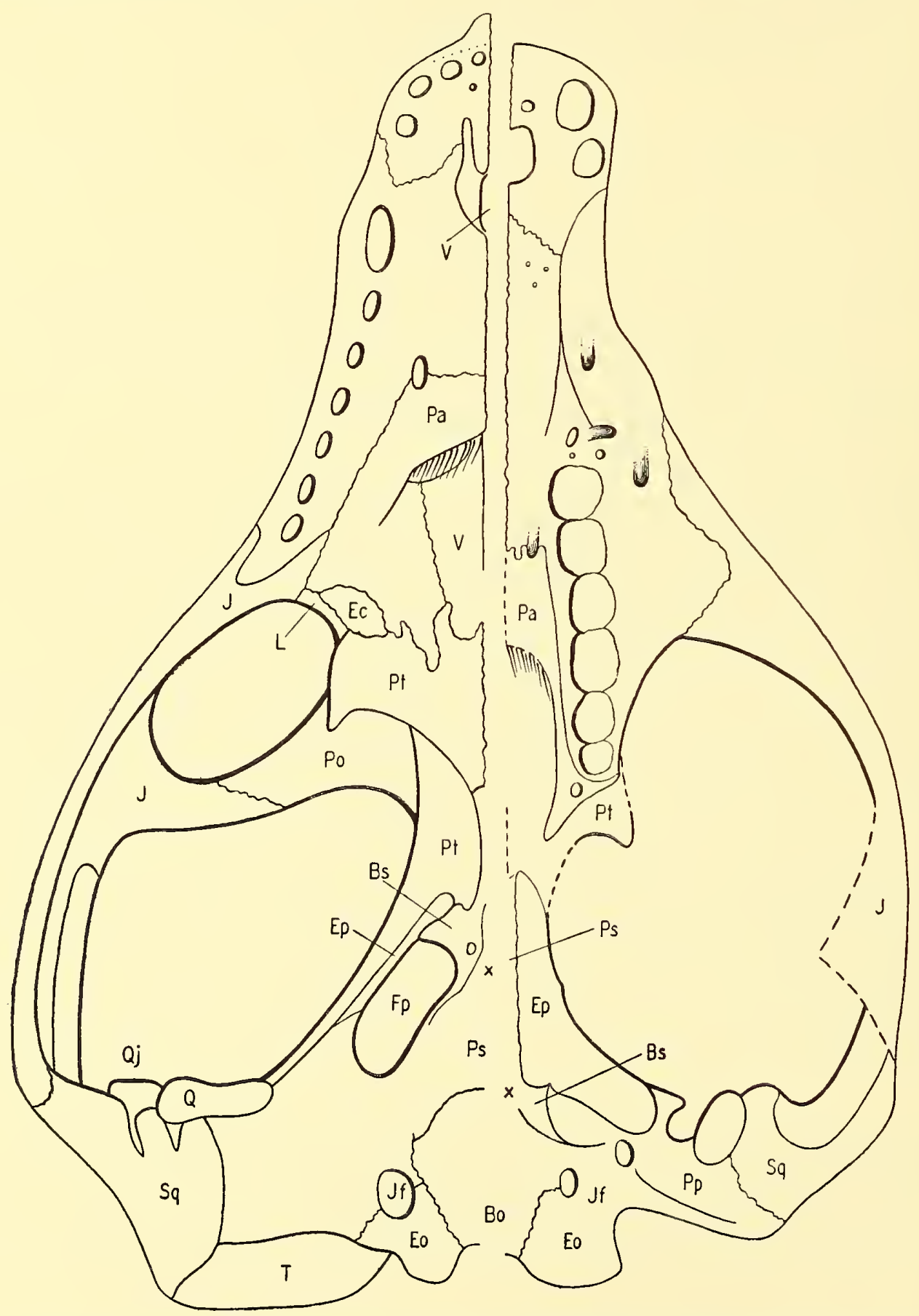

TEXT-FIG. 2I.-Comparison of the palate of Thrinaxodon liorhinus Seeley (after Parrington I946, text-fig. 2) (left) with Oligokyphus (right). Lettering as for Text-fig. I7. 
larger, the accessory bones of the jaw relatively smaller; the attachment of the quadrate to the squamosal is looser than in Cynodonts. The lateral end of the paroccipital process is hollow, the stapes lying in its concavity.

There is no indication that the quadrate and the articular complex of Oligokyphus had any auditory function. None the less, processes which would change the middle ear of

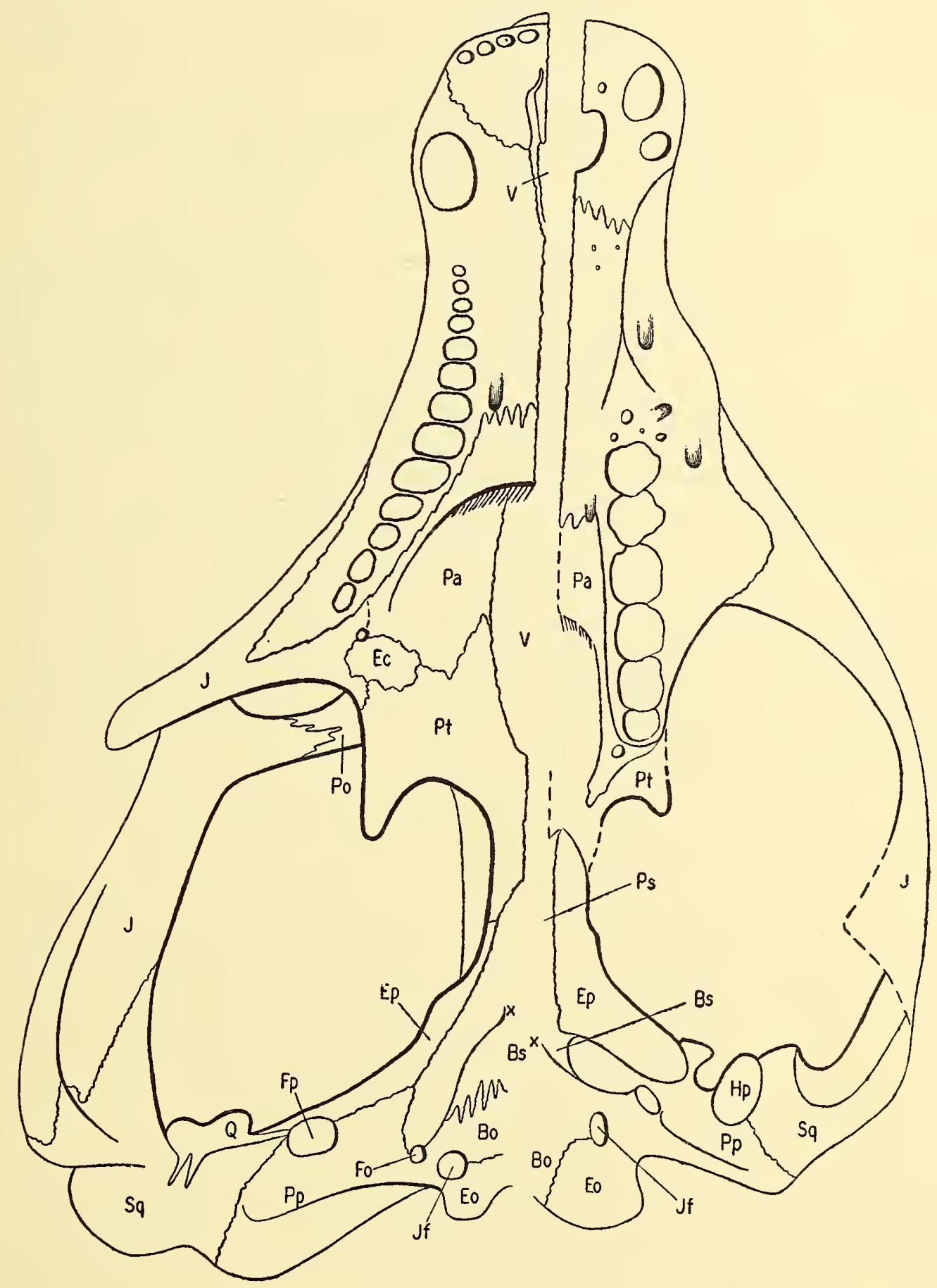

TEXT-FIG. 22.-Comparison of. the palate of. Gomphognathus cf mastacus Seeley (after Broili \& Schröder, I935, Text-fig. I3) (left) with Oligokyphus (right). 
Oligokyphus into a mammalian one can be envisaged. The dentary would gain a contact with the squamosal either anteriorly or lateral of the quadrate-squamosal contact after the height of the quadrate had decreased. In Oligokyphus the height of the quadrate is the distance which separates the squamosal and the posterior tip of the dentary.

Concomitant with an increase in brain capacity would be an overriding of the paroccipital process by the brain-case. The paroccipital process would retain its concavity, but its rim, vertical in Oligokyphus, would be turned downward into a horizontal position. Consequently the stapes would assume a vertical position. In Ornithorhynchus such a condition is realized, the crista parotica being the downturned paroccipital process. At an earlier phase the articular complex and the angular would have to lose contact with the dentary, and, together with the quadrate, would have to migrate medially until finally they found some attachment on the paroccipital process.

The establishment of the mammalian jaw articulation and middle ear necessitates morphological changes of two structural complexes, both of vital importance to the animal, to food intake and to hearing. During the whole time in which the morphological changes took place which led to the mammalian jaw articulation and middle ear, the animals concerned had to feed and to hear. Any change which impeded either function would be fatal. At least six skeletal elements are concerned in the morphological changes, which at any phase have to be correlated. The wastage of potential mammal precursors during this process must have been great. It is not at all extraordinary to find that only extremely small forms succeeded at all. Forms which-at least-had little or no "gravitational trouble". Simple characters which are characteristic of mammals do occur in the mammal-like reptiles from the Permian onwards, and often occur in unrelated families. A complex so intricate and so vital as the mammalian jaw articulation and middle ear probably evolved only once. The great importance morphologists give to this region in the Therapsid and mammalian skull would be fully justified if this were to be the case. The secondary jaw articulation and the mammalian middle ear would be truly diagnostic and their evidence would be conclusive against any other. 


\title{
THE DENTITION
}

\author{
THE UPPER CHEEK TEETH
}

(Plate II, fig. 2, Plate I2, figs. I-3 ; Text-figs. 23-28)

The form and general cusp pattern of Tritylodontid upper cheek teeth are now well known and need not be outlined again. Triglyphus, Tritylodon, Stereognathus and Oligokyphus all show them and papers dealing with upper cheek teeth by Broili \& Schröder (I936a) and Butler (I939) have made the picture still clearer. From Somerset, however, there has now come, for the first time, an abundance of Tritylodontid teeth. Though worn, the state of preservation is superior to that of Tritylodon longaeins and the Tritylodontid material at the disposal of Broili \& Schröder, and to the Chinese material (Young, I947) which does not show satisfactory evidence of the cusp pattern in a single upper cheek tooth.

The Tritylodontid dentition throws little light on the phylogeny of the mammalian dentition and hence its intrinsic interest is limited. However, the results of this investigation have the advantage of being based on ample material and are - to say the least-valid as far as this aberrant Therapsid is concerned; their importance for the understanding of late Theromorph dentitions cannot be denied. What their bearing may be on the interpretation of Mesozoic mammalian dentitions remains to be seen.

Though the cheek teeth of Oligokyphus will receive numbers and attempts will be made to compare whole dentitions of two forms differing in size as well as in the number of cheek teeth, such attempts must not be confused with the homologization of teeth of Tertiary or Recent mammals. In the latter case teeth can be identified, and where certain teeth are lost it can be shown which they are. In Oligokyphus this is not possible as its dentition is peculiar, differing from Cynodonts as well as from mammals. To avoid all commitments on the nature of the cheek teeth, which are unavoidable when using the terms " postcanine", "premolar" or "molar", it is preferable to use the neutral term "cheek tooth" when dealing with those elements of the dentition of Oligokyphus which have more than one root and are situated posterior to the diastema separating them from the incisors.

Twenty-three maxillaries are considered here, as well as eight isolated upper cheek teeth which are considerably smaller than the smallest teeth in any of the tooth-series. The figured specimens are all shown (Text-fig. 23) with a magnification of two, and those from the right side are reversed for comparison with those of the left.

Only two upper dentitions with the full number of cheek teeth are preserved-R.7038 and R.7254 (Plate II, fig. 2). Both specimens are small and the number of cheek teeth in each is six. No specimen indicates any lower number of upper cheek teeth. Not one larger dentition is complete, but three specimens, including empty alveoli as well as teeth in situ, show that the number of cheek teeth is at least seven. No specimen gives evidence of eight cheek teeth, but even the smallest specimen of the larger dentitions contains seven cheek teeth.

There are fifteen upper dentitions that yield evidence about the anterior end of the series. 


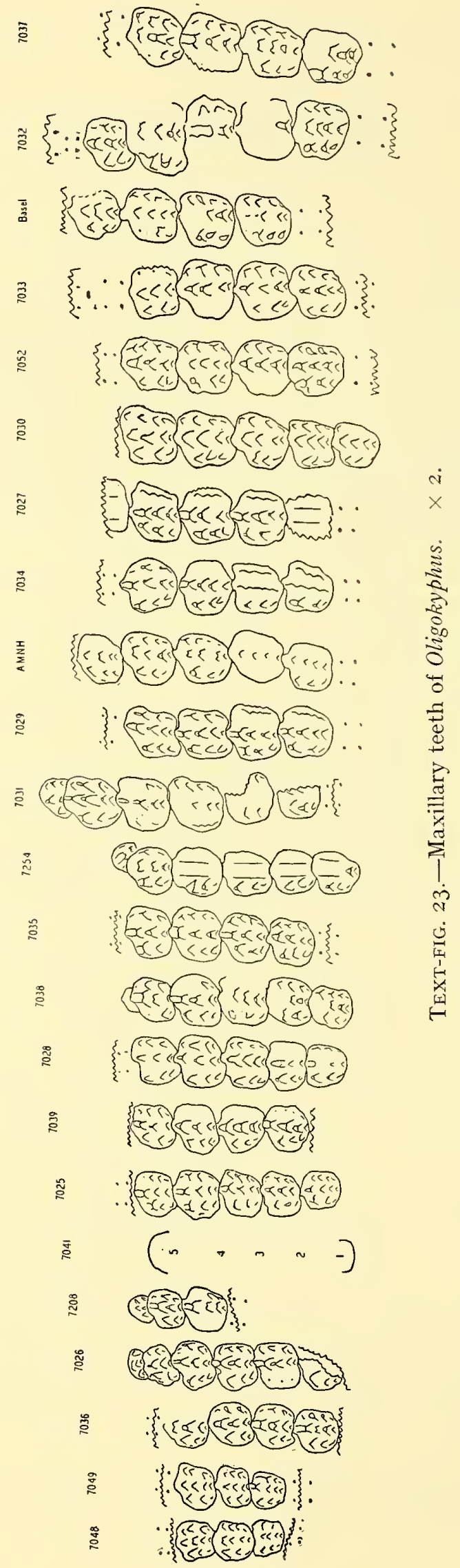


The first cheek tooth is present in five small specimens and two large ones; it has been lost before death, leaving an empty alveolus in five large specimens. Doubtful evidence of its presence occurs twice in small specimens, and of its loss once in a small and once in a large specimen.

There is no evidence, in the whole material of upper dentitions, of two cheek teeth having been lost and their alveoli still being visible. Where the first cheek tooth is present, a short period of further use would have led to its loss owing to its precarious position high above the maxillary. In several specimens the antero-external root of the first upper cheek tooth is eroded or without contact with the bone, and no alveolus is visible underneath it.

Since the maxillary is thin anteriorly to the first cheek tooth it is most likely that an empty alveolus would be obliterated in order to make the bone strong. There may therefore have been teeth in the maxillary in front of the first for which there is evidence.

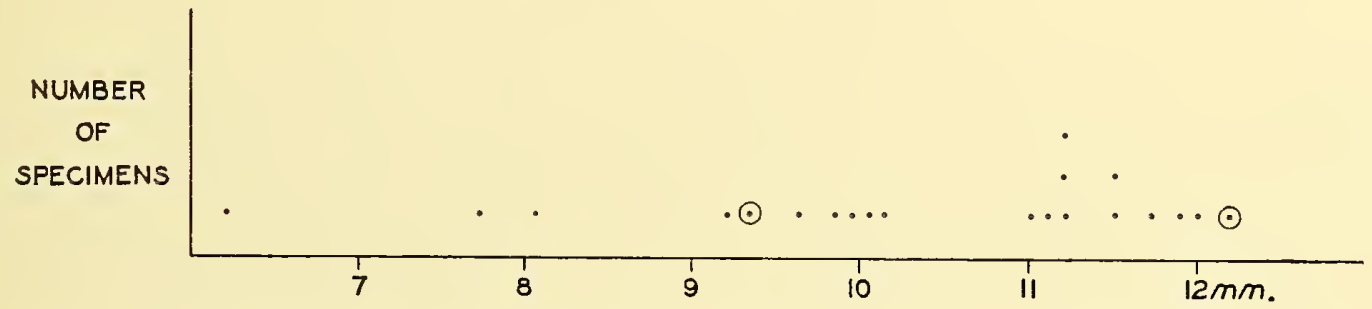

TEXT-FIG. 24.-Lengths of three upper cheek teeth of Oligokyphus. $\odot$ indicates types of 0. minor and O. major. For explanation see below.

Apart from the first upper cheek tooth which is often less worn than its successor, due to loss of the opposable tooth (R.7254, Plate II, fig. 2) the sequence of eruption can be assessed from the condition of wear. The teeth came into use one after the other from the first to the penultimate, which is always worn. The ultimate tooth is never worn though it has always emerged from its crypt when found.

In two dentitions containing either the first cheek tooth, its roots or its empty alveoli, an accurate measurement of the third to fifth cheek teeth can be made. If other specimens are graded on the assumption that the cheek teeth increase in size from in front backwards, seventeen dentitions, assumed to contain the third to fifth cheek teeth, are found. Measurements of the length of these three teeth reveal two well-defined maxima.

As will be seen later the second to fifth cheek teeth are not homologous in the dentitions containing either six or seven teeth and a similar measurement was taken of the third to fifth teeth counted from the posterior end of the dentition. This measurement includes the largest tooth in the dentitions of both size groups as well as the two preceding teeth.

\section{Length of Three Upper Cheek Teeth}

The first measurement in Text-fig. 24 is of three isolated teeth of the smallest size. Teeth No. 2-4 have been measured in the small form and teeth No. 3-5 have been measured in the large form of Oligokyphus. The dimension has been taken from the posterior margin of the posterior tooth to the apex of the anterior cusp of the mid row of the anterior tooth. 


\begin{tabular}{lclc}
$\quad$ Number & Length $(\mathrm{mm})$. & \multicolumn{1}{c}{ Number } & Length $(\mathrm{mm})$. \\
3 isolated & $6 \cdot 2$ & A.M.N.H. & II $\cdot 2$ \\
R.7049 & $7 \cdot 7$ & R.7034 & II $\cdot 2$ \\
R.7048 & $8 \cdot 0$ & R.7033 & II 5 \\
R.7026 & $9 \cdot 2$ & Copenhagen & II 5 \\
R.7025 & $9 \cdot 3$ & R.7032 & II $\cdot 7$ \\
R.7036 & $9 \cdot 6$ & R.7052 & II $\cdot 9$ \\
R.7039 & $9 \cdot 8$ & R.7037 & I2.0 \\
R.7028 & $9 \cdot 9$ & R.7030 & I2.2 \\
R.7254 & IO $\cdot 0$ & & \\
R.7035 & IO $\cdot$ I & & \\
R.7029 & II $\cdot 0$ & & \\
R.7027 & II $\cdot$ I & & \\
R.703I & II $\cdot 2$ & &
\end{tabular}

Grouping the twenty-one new measurements reveals a similar heterogeneity of the material (Text-fig. 24) though it leaves the two smallest dentitions separated from the bulk of the group of small dentitions, containing only six cheek teeth, and linking up the smallest measurement taken from three isolated cheek teeth. Discounting the two smallest maxillary dentitions mentioned above, both size-groups contain heavily worn specimens, derived from adult individuals.

As can be seen from Text-fig. 23, three longitudinal rows of cusps are a constant feature of all the teeth. The cusps are very unequal in size and, whereas the posterior cusps are

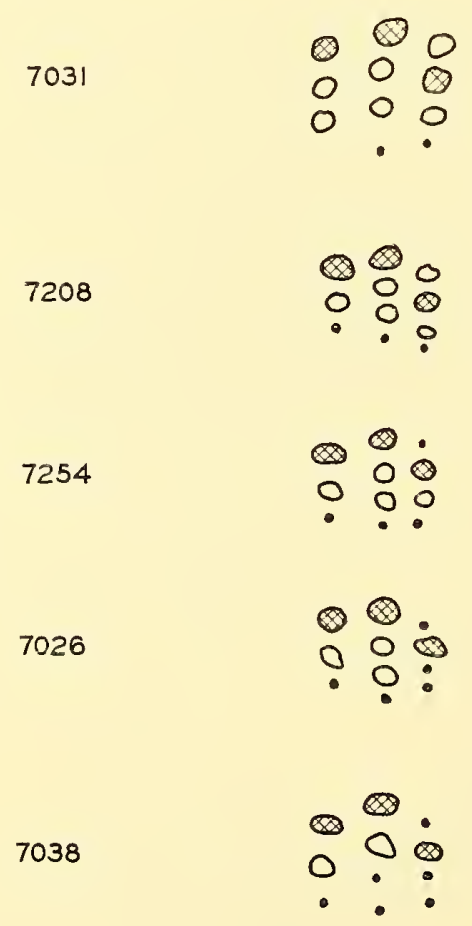

TEXT-FIG. 25.-Variation of ultimate upper cheek teeth in situ. $\bigcirc$ Cusps present on the ultimate tooth. Cusps obsolete on the ultimate tooth. $\otimes$ Highest cusp of each row. 
well-defined and obvious, the anterior cusps are very small. To regard such a small cusp as independent is necessarily a delicate and subjective matter. If, for instance, the first and second cusps of the internal row are obviously one ridge whose crest shows two distinct nicks, the conıplex is taken as two cusps.

The external row consists in all cases of three cusps, the posterior being the largest and highest. The middle row consists in general of four cusps, the posterior being the highest, though there are some specimens in the small size group, where the fifth tooth bears only three cusps in the middle row ; if this is the case, the anterior cusp becomes obsolete. The internal row of cusps invariably shows the greatest wear, and an accurate count of the number of cusps cannot be made with the same certainty as for the cusps in the middle row and the external row. 'There is evidence that all except four specimens have four cusps in this row. The exceptions are specimens of the small size group, where the fifth tooth contains in the internal row only three cusps, the anterior one being obsolete. In the internal row it is the penultimate cusp that is the largest and highest.

Thus, the evidence provided so far for morphological distinctness of the two size groups is as follows. The fifth tooth of some specimens of the small size group contains in the middle row and in the internal row three cusps instead of four, the anterior cusp being obsolete in both cases. Though very limited, this cusp reduction at the anterior end of the upper cheek teeth confirms Butler's contention derived from Tritylodon longaevus. The number of cusps in the ultimate upper cheek tooth is greatly reduced. Not only is the number of obsolete cusps much greater when compared with the fifth cheek tooth of the size-group of small dentitions, but in this tooth the cusps are obsolescent posteriorly as well as anteriorly. Five ultimate upper cheek teeth are found in situ ; there are also twelve similar but isolated teeth. Though all the ultimate teeth in situ have cut the bone enclosing their crypt, not one has reached the level of the preceding tooth. All the ultimate upper cheek teeth in situ are unworn, though the dentitions of which they are elements are very worn. This falling into disuse of the ultimate tooth has its counterpart in the lower cheek tooth dentition and is probably an indication that Oligokyphus was undergoing a reduction in the number of elements in its cheek tooth dentition.

No ultimate upper cheek tooth of a Tritylodontid has been described previously and only one is available in a large dentition (R.703I). The five ultimate teeth in situ are considered together. Of the external row consisting, in anterior cheek teeth, of three cusps, the anterior cusp becomes obsolete in the four small specimens and the full number is maintained only in the one large specimen.

Of the middle row consisting, in anterior cheek teeth, of four cusps, the first cusp is obsolete in all cases and the second is obsolete in one small specimen.

Of the internal row consisting, in anterior cheek teeth, of four cusps, the anterior one is obsolete in all cases and the second is obsolete in two small specimens. The third cusp, being the highest in this row, is present in all cases and the fourth is obsolete in three small specimens. Among the isolated cheek teeth which are referred to a similar position in the upper cheek tooth dentition, the posterior cusp of the internal row is obsolete in five cases out of eleven.

It is in the obsolescence of this cusp, which will be referred to again, that the reduction of elements at the posterior end of the crown of the upper cheek teeth is observed. This great variability of the ultimate upper cheek tooth is in striking contrast to the condition 
found in the preceding teeth. The degree of cusp reduction in the ultimate upper cheek tooth is greatest in the internal row, less in the middle row and least in the external row.

Among the large number of isolated upper cheek teeth there is no single specimen which, on morphological grounds, could be placed posterior to the tooth described here as the ultimate.

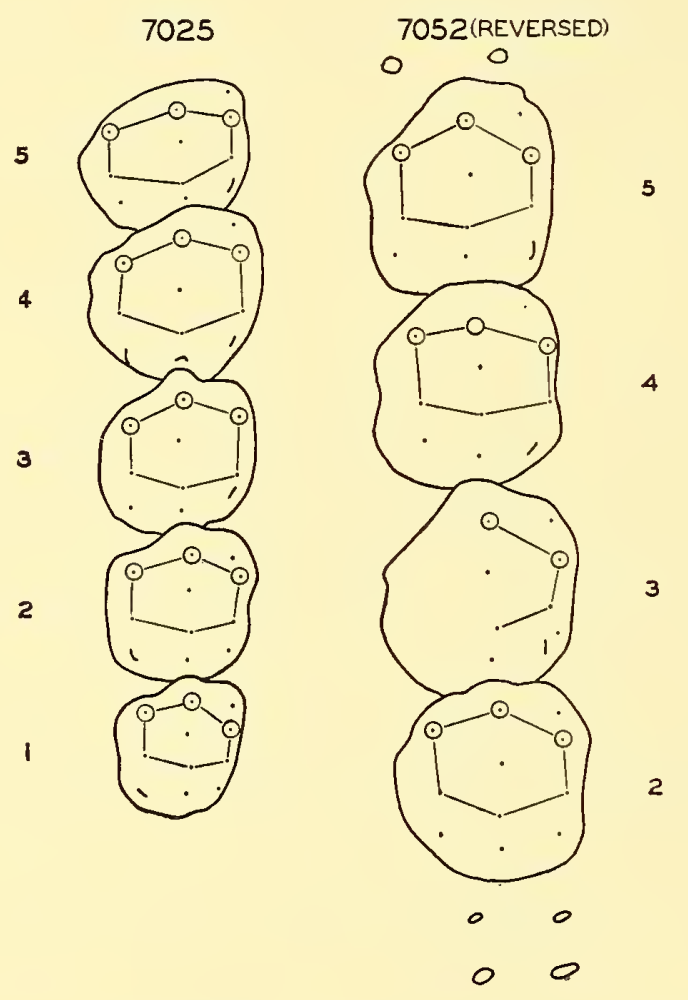

TEXT-FIG. 26.-Variation of cusp pattern along upper dentition of $O$. minor and $O$. major

Of the five specimens which contain the ultimate upper cheek tooth in situ, four belong to the small size group. The evidence makes it unlikely that there could have been a still smaller tooth behind the ultimate, and no such tooth has in fact been found.

On the other hand, the ultimate tooth of the size group of larger specimens has only once been found in situ (R.703I) and, as can be seen from Text-fig. 25, it is less reduced than that of the four smaller specimens. The maxillary bone is not preserved posterior to the last cheek tooth in R.703I ; thus there is no evidence for the presence or absence of a still smaller tooth behind the one described as the ultimate. Furthermore, since they occur similarly in the dentary, there may also have been two very much reduced posterior cheek teeth in the maxillary of the size-group of large specimens. The apparent absence of such a tooth among the isolated teeth can be explained by maintaining that the great number of isolated ultimate upper cheek teeth contains the ultimate tooth of small specimens, the seventh tooth of large specimens (described above as the ultimate) and the eighth tooth of large specimens which is indistinguishable from the ultimate tooth of small specimens.

However, until an eighth upper cheek tooth has been found in situ, there is no evidence to support these speculations and they are not taken into account. 
UPPER CHEEK TEETH; SUCCESSION OF CUSPS

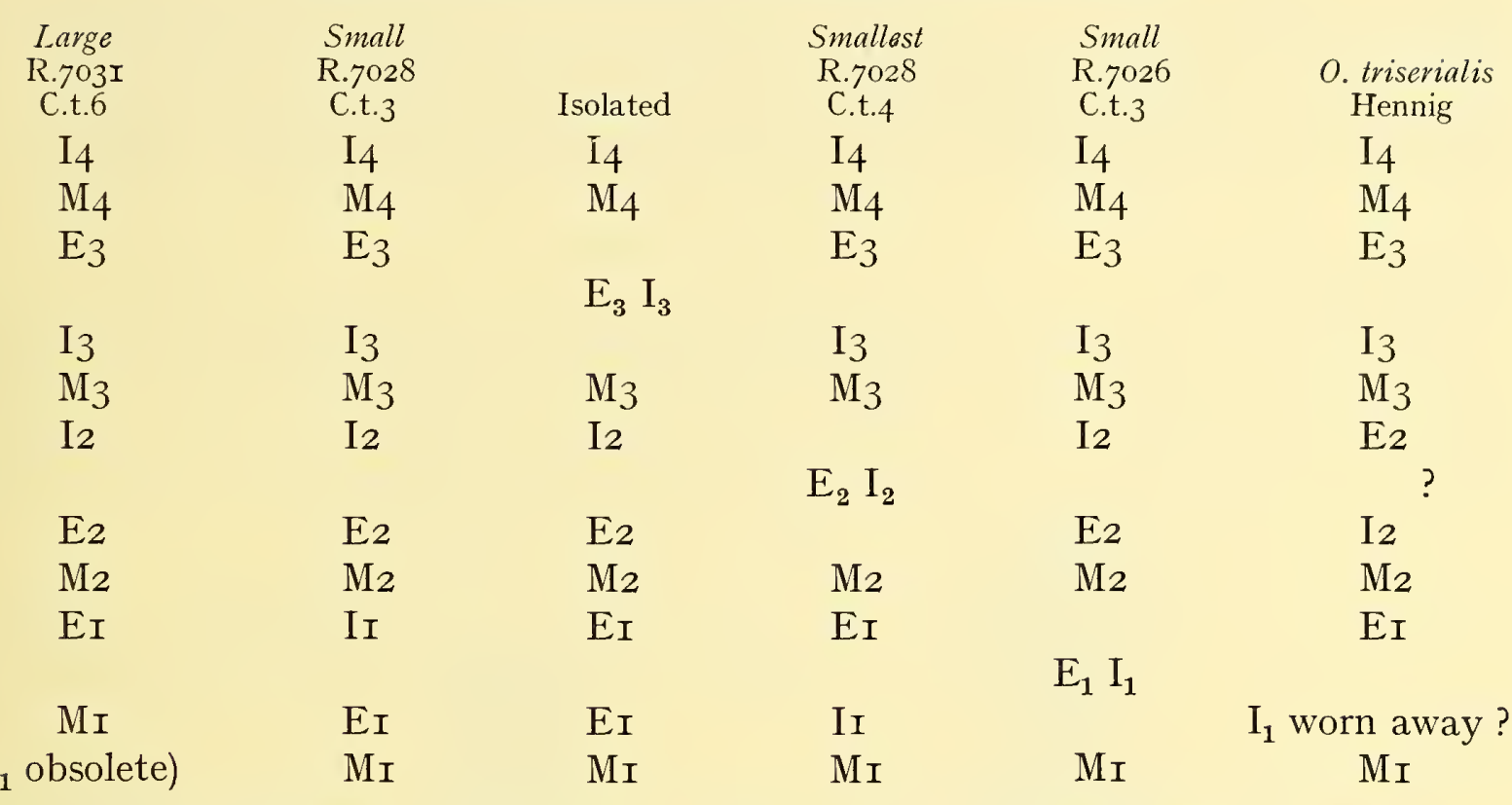

Explanation of symbols used : $\mathrm{I}=$ cusps of internal row ; $\mathrm{M}=$ cusps of middle row ; $\mathrm{E}=$ cusps of external row. Numbers from front to back.

The arrangement of cusps in normal unreduced cheek teeth is illustrated by Text-fig. 27 and in the adjoining table. From these it is evident that the mutual relationship of all the
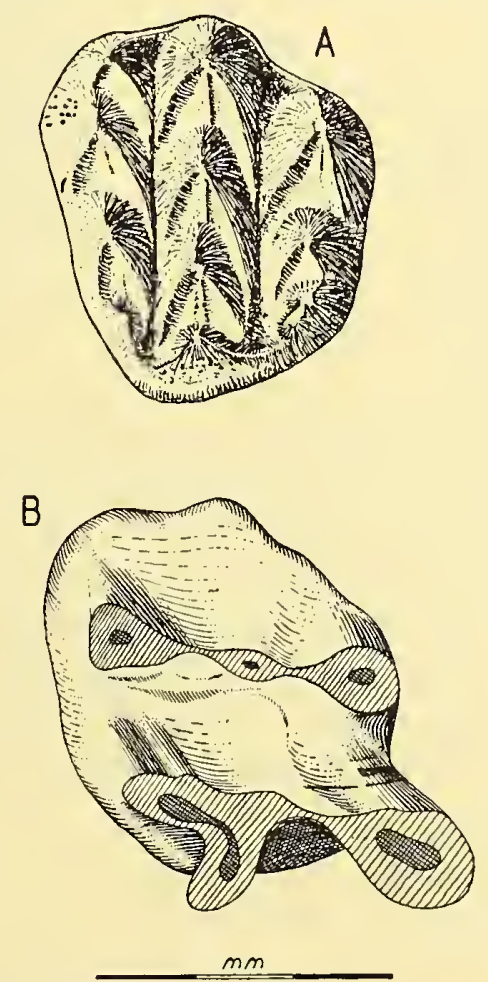

TEXT-FIG. 27.-Upper cheek teeth of Oligokyphus. A, Right, crown view of R.7256.

B, Left, dorsal view of R.7266. 
cusps is constant, that is to say, the succession of cusps is practically identical along the dentition as well as in both size-groups. Minor differences in the cusp succession are borne out by varying hexagons which connect six well-developed cusps of the posterior part of the cheek teeth in one representative of each size-group (R.7025 and R.7052). Rarely are there two cusps on the same level.

\section{The Shape of Cusps}

Plate I2, fig. 3, above, and Text-fig. 27 represent the tooth already figured in a preliminary account (Kühne 1943, fig. 2A). This figure has been carefully revised and is now almost perfect. The second cusp of the middle row has been restored as previously. The principal alteration in the drawing is the pushing back of the third external cusp so that it is behind the level of the third internal cusp, which is its correct position.

As will be seen, an upper cheek tooth from the anterior or middle part of the dentition contains eight cusps which bear anteriorly the typical concave crescent face ; two cuspsthe internal and external anterior-are merely ridges, and one cusp-the anterior of the middle row-has a posterior face like those of the succeeding cusps, but its anterior face is wrinkled and not smooth and concave.

Cusps $\mathrm{I}_{2}$ and $3, \mathrm{M}_{2}, 3$ and 4 , and $\mathrm{E}_{3}$ have the concave crescent face subdivided longitudinally by a faint central rib which is low and which merges smoothly into it on both sides. The asymmetry of the crescents of the cusps of both the internal and external rows has often been referred to. This asymmetry is exaggerated by wear and all Tritylodontid teeth previously described have been worn. In unworn upper cheek teeth the external rim of the external cusps does not reach as far down as does the medial rim of the same cusp and thus the appearance of asymmetry is produced. This also accounts for the asymmetry of the crescent faces of the cusps of the internal row.

A distinct supernumerary cusplet on the buccal side of the external row of some specimens is located at the anterior end of the lateral edge leading away from the apex of $\mathrm{E}_{3}$, which is the largest and ultimate cusp in the external row. There is only one tooth in the sizegroup of large specimens which has such a cusplet and it occurs in the sixth tooth of a specimen in the American Museum of Natural History. As will be seen in due course, the sixth tooth in large specimens is homologous with the fifth tooth in small specimens. The same cusplet occurs once only in the second tooth of the very small specimen R.7049, three times in the fourth tooth and twice in the fifth tooth. There are numerous isolated posterior upper cheek teeth which have the same cusplet and its significance is doubtfui. It must be functionless as it cannot occlude any element in the lower cheek tooth dentition.

Plate 12 and Text-fig. 28 show that the internal row is nearer to the middle row than is the external row and it is not so deep. The internal row also is peculiar in having its ultimate cusp smaller than the preceding cusp. Apart from this, the apex of its ultimate cusp is medial to a line connecting its predecessors and its medial edge actually obstructs the propalinal motion of the opposing cusp in the lower cheek tooth, until it is worn away. However, this is not a serious maladaptation as in occlusion the smallest cusp of the lower cheek tooth, which is narrower than its predecessors, is in contact with it.

Features which can only be observed on unworn teeth are the edges which lead up to the apices of all three cusps from the posterior margin of the tooth. The edge to the posterior 
cusp of the internal row runs along the posterior margin of the tooth and enters the buccal side of this cusp. The same applies to the posterior cusp of the middle row. The edge to the posterior cusp of the external row enters the lingual side of this cusp. Though these edges actually form the posterior margin of the tooth, between the cusps, they are almost on a level with the bottom of the two grooves between the three rows of cusps; the edges do not close the grooves posteriorly in such a way that the latter could be termed basined. Anteriorly the longitudinal grooves in the upper cheek teeth are well closed and basined. One ridge connects the external and middle row and another, originating from the anterointernal cusp, joins this cusp with the antero-central. The anterior ridges, closing the longitudinal grooves, obstruct the propalinal motion of the lower cheek teeth and can only

A

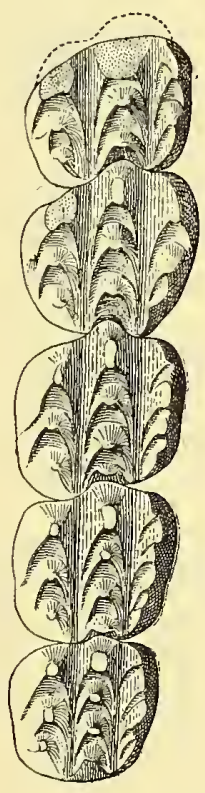

B

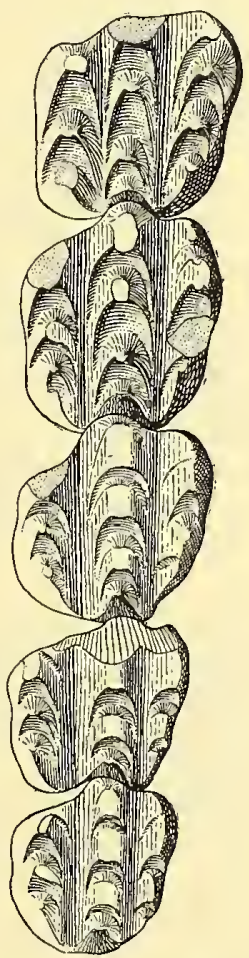

TEXT-FIG. 28.-Two left upper dentitions. A, R.7025, holotype of Oligokyphus minor. B, R.7030, holotype of Oligokyphus major. The ultimate cheek tooth is missing in R.7025, the ultimate and the penultimate tooth are missing in R.7030. Both dentitions are worn.

be observed in slightly worn or unworn teeth. For instance, R.7256 (Text-fig. 27, A) still shows the basins perfectly though the tooth is a little worn ; it is not unworn as was stated in a preliminary account. Actually unworn upper cheek teeth are, with the exception of the ultimate tooth, exceedingly rare and only one isolated specimen, R.7257, has been noticed.

\section{Roots of Upper Cheek Teeth}

The roots of the upper cheek teeth are in two transverse rows under the crown (Text-fig. 27). These rows are always well separated and never connected by thin web-like sheets of dentine. The anterior row consists of a round external root well separated from the large and 
\rfloor -shaped internal root, which actually supports the internal as well as the middle row of cusps. Posteriorly there are three roots, the one under the middle row of cusps being by far the smallest. Each transverse row of roots may be joined together by thin web-like sheets of dentine, especially near the crown.

In the ultimate upper cheek tooth the two transverse rows of roots are closely apposed and single roots cannot be observed in many of the isolated specimens.

\section{Dimensions of Upper Cheek Teeth}

The length of each tooth can only be measured if the tooth is isolated, for the cheek teeth are imbricated in such a way that the posterior cusp of the middle row of each tooth overhangs the anterior cusp of the middle row of the succeeding tooth, which is thus hidden from ventral view. The breadth, however, can be measured easily in associated dentitions and is taken at right angles to the two longitudinal grooves which divide the three rows of cusps. Text-fig. 23 shows that the size of the upper cheek teeth increases from the first to the second before the last. The penultimate tooth is slightly smaller than its predecessor and the ultimate cheek tooth is considerably smaller. In the group of large specimens with seven cheek teeth we have, therefore, five teeth increasing in size; and in the group of smaller specimens with only six cheek teeth, four teeth increasing in size; followed in both cases by one tooth slightly smaller than its predecessor and another tooth, the ultimate, very considerably smaller.

In the size-group of smaller dentitions with six cheek teeth, the breadth is consistently slightly less than the length. With increase in size the teeth become more elongated (Text-fig. 39).

\section{THE LOWER CHEEK TEETH}

(Plate I2, figs. I, 4 ; Text-figs. 29-32)

The lower cheek teeth of Tritylodontids are still little known. The first lower cheek tooth of a Tritylodontid to be described was Oligokyphus biserialis, discovered in IgoI and described by Hennig in 1922 as an upper premolar, and finally recognized by Simpson in 1928. Simpson's finding was corroborated when Young, in his preliminary paper on Bienotherium (I940), gave descriptions and drawings of lower cheek teeth. During Dr. Young's visit to London in 1945 it was possible to prepare three lower cheek teeth which, being unerupted posterior elements of the tooth row, had escaped wear and subsequent damage and are the only cheek teeth of Bienotherium which show their morphology. Unfortunately, Dr. Young had no time to take cognisance of the new preparations and the figures of them by Miss J. Townend are not referred to in his text. (See Young, I947: 556, text-fig. 7 (a) ; 564, text-fig. II.)

Young's text-figure IIA of specimen V.5 shows how deep the posterior cheek teeth are below the level of the preceding teeth. The condition is actually the same as in Oligokyphus (R.7280 in Plate I2, fig. 4, right).

The author's figures (Kühne, I943, fig. 2 B, C, D) of the Jnwer cheek tooth, R.7278, are typical for elements from the middle of the dentition. The lateral view, however (fig. 2C), was slightly incorrect and is emended in the new Text-fig. 29, D.

The lower cheek tooth dentition consists anteriorly of smaller elements, the middle teeth 
are "typical" and at the end of the tooth row progressive reduction of its elements takes place.

In specimen R.7278 (Plate I2, fig. I, right: Text-fig. 29, B, D, H) the lateral and medial sides of the tooth are nearly straight and parallel. The anterior width of the tooth is, however, slightly greater than the posterior. Anteriorly the margin of the tooth is concave, the convex posterior margin of the preceding tooth fitting tightly into the concavity.

The crown consists of two longitudinal rows of cusps, the internal row slightly narrower and lower than the external one. Each row consists of three well-defined cusps, the anterior always being the largest and highest and the posterior the smallest. The first two pairs of

$\wedge$

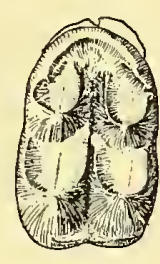

$B$

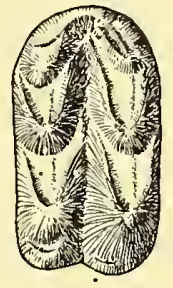

c

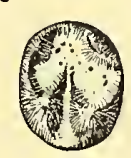

D

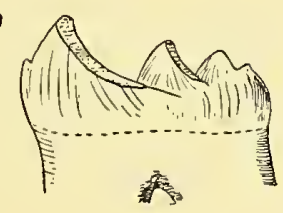

E
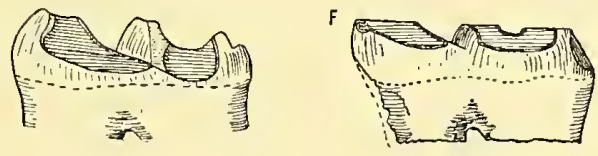

G
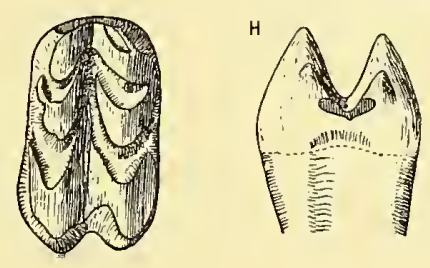

s

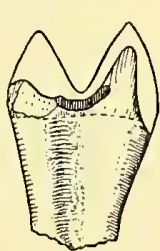

k

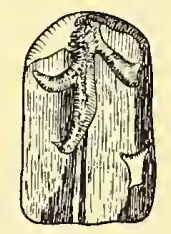

M

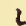

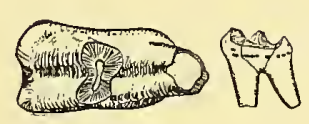

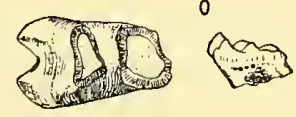

TEXT-FIG. 29.-Isolated lower cheek teeth of Oligokyphus. In crown view the anterior end points down, in side view the anterior end is left. Scale in mm. A, R.7279, large right, unworn, isolated fifth cheek tooth; crown view. B, R.7278, large, right, unworn, isolated cheek tooth, slightly worn; crown view. The drawing is reversed and depicts a left tooth. The letter B is near the internal side of the tooth. C, Right ultimate lower cheek tooth in the large dentary R.7II7. D, R.7278, medial view. E, Arterior left cheek tooth of R.7I57; lateral view. F, R.7286, heavily worn left cheek tooth ; lateral view. G, R.7286 ; crown view. H, R.7278, right ; anterior view. J, R.7288, left ; anterior view of heavily worn tooth. K, R.7387, cheek tooth showing extreme wear in crown view. L, R.7283; anterior cheek tooth in ventral view to show form and arrangement of roots; the small side view not to scale. M, R.7285; cheek tooth with well-preserved roots in side view, in half the magnification of the other teeth. N, R.7283; large posterior right cheek tooth; ventral view showing roots in half the magnification of the other teeth. o, R.7283, medial view, not to scale. 
cusps have a convex anterior face and a deeply concave and crescentic posterior face. Both faces of each cusp are well separated by two ridges which begin at the apex and diverge, the lateral ridge fading out at the margin of the tooth and the medial ridge ending in the deep longitudinal groove between the rows of cusps. The concave crescent faces are longitudinally divided by a smooth ridge. Anterior to each first cusp there is a very small accessory cusp, the internal one being more distinct and a little larger than the external. The posterior end of the crown is formed by a pair of small cusps which have a typical convex face anteriorly, as have the preceding cusps ; their posterior faces, however, are not concave and crescentic, and there may be only one ridge or two, very near together. From the external ridge of the posterior cusps, a ridge may develop running round the posterior margin of the tooth and forming a definite basin, or the posterior ridge may be independent of the posterior cusps. There is no basin at the anterior end of the tooth.

The lower cheek teeth each have two roots, one behind the other. The anterior root is $\infty$-shaped in transverse section, its long axis being transverse; the posterior root is round. Thus the alveolus of an anterior root can easily be distinguished from that of a posterior root, a point of importance if the former presence of teeth is inferred from the presence of roots or empty alveoli.

The material of lower dentitions is more numerous and qualitatively superior to that of the upper cheek teeth. Many complete dentitions are available, and there are also juvenile stages. The relationship of the teeth to the dentary can be defined with the help of the posterior corner of the alveolar border, the anterior end of the masseteric fossa, the posterior end of the symphysis, and the mental foramen. Text-fig. 30 shows the distribution in size of the whole material : there is a group of very small specimens, all of which show in their lack of a partition of the crypt of the ultimate tooth that the latter had not yet developed roots. In the fully grown tooth the partition separates the anterior from the posterior root. There is another rather compact group of small specimens, all but one being adult. There is finally a less well defined group of large specimens, all but two being adult.

Text-fig. 3I shows twelve reasonably complete dentitions arranged according to their length. They show an astonishing degree of variability. The distance between the mental foramen and the end of the alveolar border ranges from 16 to $33 \mathrm{~mm}$. The distance between the mental foramen and the posterior end of the symphysis ranges from o to $4.4 \mathrm{~mm}$. The distance from the mental foramen to the anterior end of the masseteric fossa ranges from 9 to $20 \mathrm{~mm}$. The distance from the anterior end of the masseteric fossa to the posterior end of the alveolar border may be from 7.5 to $15.5 \mathrm{~mm}$. On the other hand, the length of the three largest teeth available in the specimens is only from ro to $13.5 \mathrm{~mm}$.

As the row of cheek teeth functions as a unit, the cheek teeth never lose touch, hence increase of length of the dentary can only take place in front and behind the row.

\section{Number of Lower Cheek Teeth in the Large Adult Form}

The eight dentitions in Text-fig. 3I are considered here. Specimen R.7133 is broken off in the middle of the tooth row. On a level with the mental foramen there is an anterior root in situ, followed by a posterior root and two other teeth, of which only the roots are present. The posterior of these two teeth, which is the third tooth of which there is evidence, has its anterior root on a level with the posterior end of the symphysis. This tooth was lost 
prior to fossilization. There follow three teeth, increasing in size, and a pair of roots for a tooth obviously functional but lost due to predator action; the fracture at the hinder end of the specimen halves the posterior alveolus of the lost tooth.

R.7IIg has five functional teeth which are preceded by roots of one tooth situated behind the posterior end of the symphysis. Posteriorly there is one rather large tooth unerupted.

R.7I22 has five functional teeth (the two anterior teeth were lost when the specimen was exposed) and posteriorly one unerupted tooth.

R. 7I2I has five functional teeth preceded by a pair of alveoli, the anterior alveolus being on a level with the posterior end of the symphysis. Posteriorly there is one unerupted tooth and behind it is a vestige of a tooth measuring $0.2 \times 0.2 \mathrm{~mm}$. and consisting of only one cusp.

R.7I34 has five functional teeth preceded by a pair of alveoli in the same position as in R.7I2I. Posteriorly there is an unerupted tooth. This is also the case in R.7I23.

R.7I80 has five functional teeth, the first on a level with the posterior end of the symphysis and one tooth is unerupted posteriorly.

Finally, R.7372 has five functional teeth, the first on a level with the posterior end of the symphysis and posteriorly two teeth unerupted.

No replacement and no shedding of any cheek tooth has ever been observed.

As anterior cheek teeth are morphologically identical with posterior elements of the tooth row and wear decreases posteriorly, all cheek teeth must belong to the same dentition and it would therefore be wrong to call anterior cheek teeth " premolars".

There is one instance of three pairs of roots in front of the first functional cheek tooth (R.7133).

There are three instances of one pair of alveoli in front of the first functional cheek tooth (R.7I34, R.7I2I, R.7IIg), the anterior alveolus being a little behind the posterior end of the symphysis.

There is one specimen without an alveolus in front of the first functional cheek tooth, the latter being well behind the posterior end of the symphysis (R.7I22).

There are two specimens, each with five functional teeth and no alveolus in front, the anterior functional tooth being on a level with the posterior end of the symphysis (R.7I80 and R.7372).

The anterior functional cheek tooth in these two specimens is here called the "zero" tooth (=0 in Text-fig. 3I) and it is homologized with the pair of alveoli available in R.7I34, R.7I2I and R.7II9. Counting backwards from No. I, R.7372 has six teeth, the last two unerupted ; R.7I80 (Plate II, fig. 5) has five teeth, the ultimate unerupted (the absence of a sixth tooth is regarded as "abnormal ") ; R. 7I23 (Plate II, fig. 3) has six teeth, the ultimate unerupted; the same observation applies to R.7I34 and R.7I2I, the latter having behind the sixth tooth the vestige mentioned above. Specimens R.7I22 and R.7IIg each have six teeth, the ultimate unerupted. The occurrence of a functional "zero "would be an indication of a youthful stage, which is corroborated in R.7372 where No. 5 is still unerupted. The occurrence of two pairs of roots in front of " zero " in R.7I33 also indicates a rather youthful stage and shows the presence of at least two anterior teeth preceding a phase in the lifetime of the animal, when further loss of anterior teeth was retarded. There is no large specimen where tooth No. I is already lost.

We have, therefore, in the dentary of the large adult form of Oligokyphus five functional 
teeth, succeeded by one tooth which is permanently unerupted. In front there is the "zero" tooth, twice retained, four times lost and once lost so early that no trace of it is seen on the alveolar border, which may be a sign of old age (R.7I22). In Text-fig. 30 "zero" is counted together with the functional and unerupted teeth to make a total of seven teeth.

The two posterior cheek teeth are reduced in size and in number of cusps and their morphology is described here: No. 5 is seen in the perfect isolated specimen R.7279, which appears in crown view in Plate I2, fig. 4 and Text-fig. 29, A; the third posterior pair of cusps is almost completely reduced and is only indicated by a faint thickening of the marginal edge which forms the posterior basin of the tooth. This specimen is also noticeable as one

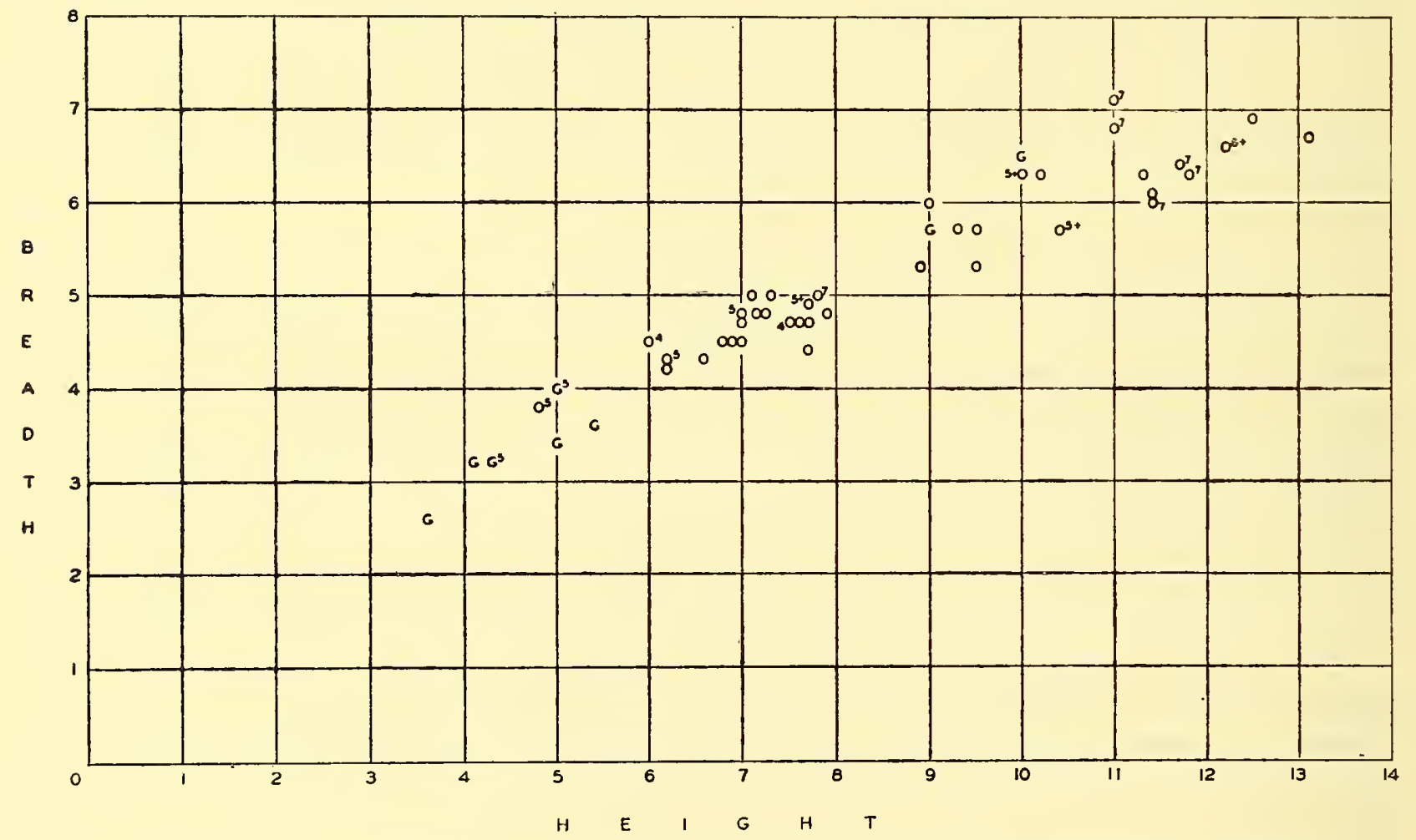

TEXT-FIG. 30.-Oligokyphus. Distribution and size of lower cheek teeth. Dots only: specimens without teeth or with fragmentary dentition; Dots and numbers: specimens whose complete dentition is known, the number of teeth being indicated; $G$, specimens not fully grown; $G$ plus number, growing specimens with their complete dentition.

of the few unworn lower cheek teeth, and for the narrowness of the longitudinal groove between the internal edges of the first pair of cusps. This last feature is such that the apex of a cusp of the middle row of the upper cheek tooth cannot reach the bottom of this groove unless considerable wear has already taken place. It means that in the unworn condition an optimal functioning of a cheek tooth is impossible. In No. 6 (Text-fig. 29, c) the process of reduction has eliminated the posterior pair of cusps and the middle pair is now in the condition of that of the posterior pair in tooth No. 5. Wherever this tooth is present it has been made visible by dissection. The anterior cusps of the tooth are not on the same level, the internal cusp being displaced anteriorly. The long axis of the tooth often does not coincide with those of the preceding teeth. 
Juvenile Stages

R.7r36 (Plate I2, fig. 5a) and R.7I5I are juvenile specimens, indicated in Text-fig. 30 by "G" (for "still growing") and both have lost their posterior teeth by predator action. Both specimens show that the roots for the cheek teeth Nos. 5 and 6 were not yet formed when death occurred, because the crypts, in both of which the lateral wall is preserved, are still undivided by a partition which separates the anterior from the posterior alveolus in fully formed teeth.

\section{The Small Adult Form}

In the group of smaller adult forms, of which four specimens are shown in Text-fig. 3I, few other differences are found, apart from smaller size and a smaller number of cheek teeth. The tooth pattern is identical with that of larger specimens of similar position. However,

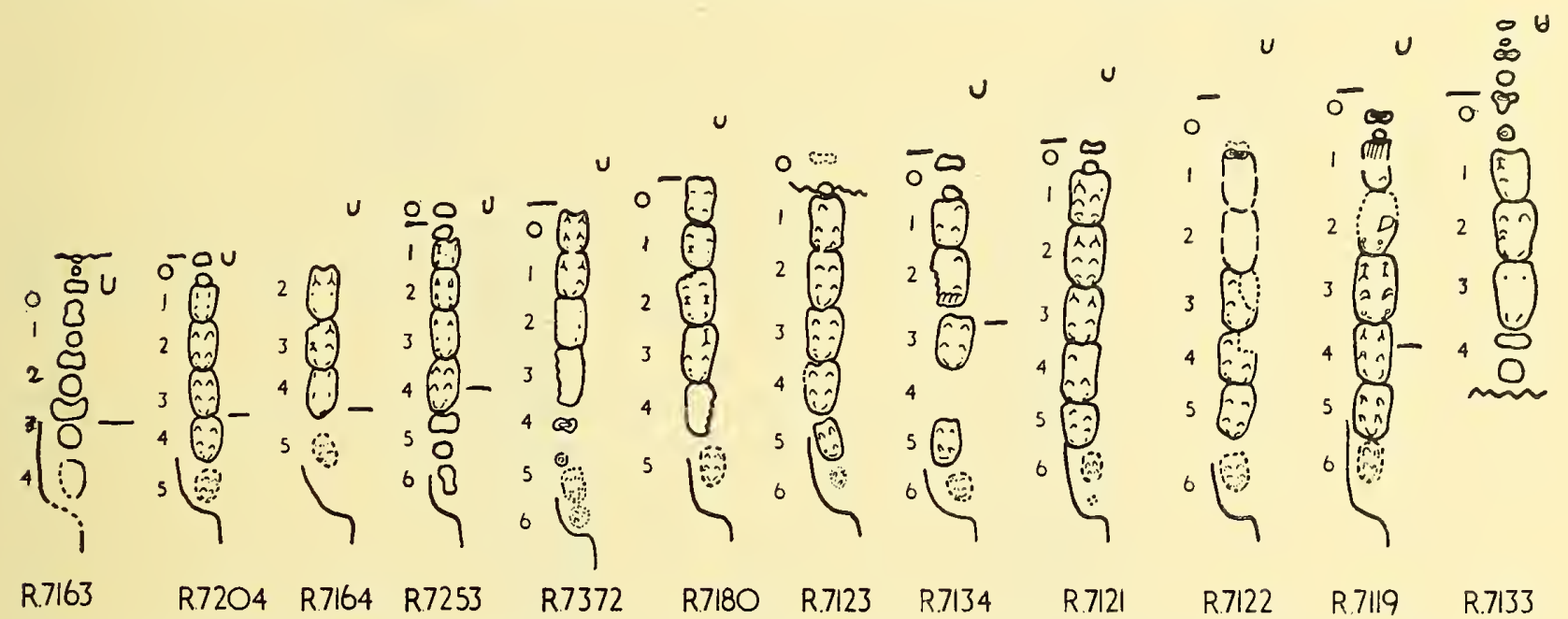

TEXT-FIG. 3I.-Lower cheek teeth. $\times$ I.5. Only fairly complete dentitions have been figured. Specimens from the left side have been reversed. The lateral side of each specimen is thus on the right. Increase in size from left to right. Key : $\sim=$ fracture. - (anterior, front) $=$ posterior end of symphysis. - (posterior, left) = anterior end of masseteric fossa. $\mathrm{U}=$ anteriorly, external ; mental foramen. $O=$ outline of teeth dotted; unerupted teeth. $O=$ roots of teeth, or if anteriorly, indication of tooth once present.

the presence of two teeth which are considerably smaller in size and have a reduced number of cusps, at the posterior end of the dentition, is not general in the small adult form. There are only two such specimens in a total of fifteen which have the relevant region preserved; they are R.7253, where only the undivided crypt of the ultimate tooth and the alveolus of the penultimate tooth are seen (Text-fig. 3I), and R.7173, where there are both teeth. Anterior to the first functional tooth there are in R.7204 (Plate 4, fig. I $b$; Plate II, fig. 3) and R7253 a pair of alveoli which are homologized with the "zero" tooth in the larger adult form. R.7I64 is interesting in showing a complete functional dentition of only three elements; its ultimate cheek tooth is not yet erupted and "zero" and No. I have been lost ; though the former presence of No. I is indicated by pressure wear on No. 2.

Only one specimen of this size group shows posteriorly the sign of juvenility. This is R.7I63 (Plate I2, fig. $5^{b}$; Text-fig. 3I) ; it lacks all teeth, but there are six alveoli. The pair of alveoli, on a level with the mental foramen are regarded as those of a " non-permanent" 
cheek tooth, and are followed by "zero " and four more teeth ; the ultimate in the series is shown only by the lateral wall of its crypt, which is not yet subdivided to receive the two roots of this tooth. R.7I67 and R.7I68 give evidence as to this. The number of cheek teeth in the small adult form is more variable and also more doubtful than in the large adult form. Though the total of observable teeth may be as little as four and as high as seven, the number of functional cheek teeth appears rarely to exceed four, a number which may be corroborated by more and better material. It is certainly rare to find an unerupted posterior cheek tooth, though the specimens in Text-fig. 3I show it, for comparison, in all the large adults. It should be borne in mind that some juvenile specimens are only slightly smaller than the adults, for this is essential to an understanding of the following discussion.

\section{The Five Smallest Dentaries (Plate I2, fig. 5; Text-fig. 30)}

The five smallest dentaries in Text-fig. 30 are regarded as juvenile for two reasons; the crypt of their ultimate cheek tooth is always undivided (a feature which has already been observed in three specimens of the two full sized groups) and the bone is characteristically sculptured, for instance at the masseteric fossa and at the angular corner.

R.7I7I and R.7I72 are fragments of the posterior part of the dentary without teeth. R.7I69 agrees in size with R.7I88 and R.7252 and contains one cheek tooth in situ. This tooth is in the posterior half of the row of cheek teeth and is only $2.5 \mathrm{~mm}$. long. It is neither in the position of "zero" nor is it the tooth anterior to "zero" teeth which in the small adult form would be of similar size.

There are also five very small isolated lower cheek teeth, three of which are $2.5 \mathrm{~mm}$., $2.0 \mathrm{~mm}$. and $2.0 \mathrm{~mm}$. long. Their dimensions are such that they would fit the three smallest dentitions. The five teeth are worn and have well-developed roots.

A posterior tooth, much smaller than those in the small adult form, may either indicate another minute species of Oligokyphus or the presence of a milk dentition. As it is found in a manifestly juvenile dentary, the latter is the more likely. Unfortunately there is no other corroborating evidence regarding tooth replacement in Oligokyphus.

\section{SUCCESSION AND WEAR OF TEETH}

(Plate I, fig. I $a$, Plate II, fig. 2, Plate I2, fig. 2 ; Text-figs. 28, 29)

The lower dental series show that the degree of protrusion of a tooth decreases posteriorly. Teeth which have been anterior to the first functional tooth are lost because the amount of root above the alveolar border is such that each tooth is no longer in a stable position, and becomes therefore broken off at the alveolar border. When in the large dentary tooth "zero" has been lost, no further loss is incurred and the dentition is stable so far as the number of its elements is concerned. The eruption of the last functional cheek tooth is decidedly later than that of its predecessors and in several instances this tooth is hardly worn. The ultimate upper cheek tooth erupts but is never in use, and the ultimate lower cheek tooth of the large adult form never erupts.

Plate II, fig. 2 and Text-figs. 28,29 show the kind of wear that occurs. There is no wear, of course, on the lateral and medial side of the upper cheek teeth. An initial stage of wear is realized if the enamel is nowhere worn through, consequently the edges of the cusps are sharpened and the efficiency of the tooth is increased (R. 7258, Plate I2, fig. 2). Wear 
facets which do not penetrate the enamel are always straight and horizontally striated. Once the thin enamel is worn through, the dentine is often scooped out between high ridges of enamel, and when the enamel has vanished from the apex of a cusp the enamel margins of the anterior and posterior slope of the cusp are actually higher than the apical area. In such a case, if the preservation of the tooth is not very good, the number of cusps present
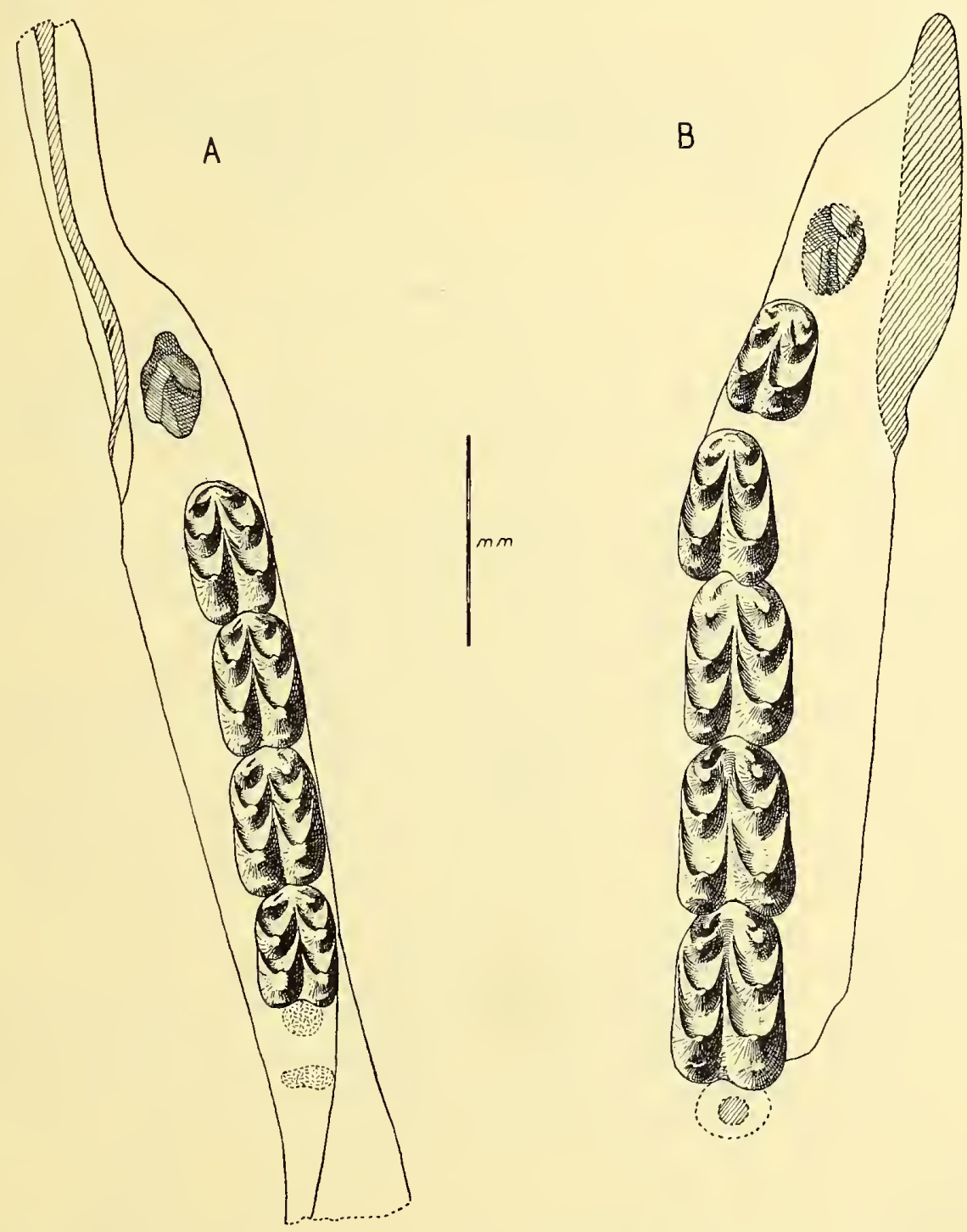

TEXT-FIG. 32.-Lower cheek teeth. A, R.7204, Oligokyphus minor, right. B, R.7I23, Oligokyphus major, left. The ultimate cheek tooth in both specimens is still unerupted. The anterior tooth zero has been lost in R.7204 and the alveoli are already closed. The same tooth is missing in R.7123, due to fracture.

in the tooth may be uncertain. Broili \& Schröder (I936) have already mentioned this. The final stages of wear, when the morphology of the cheek tooth is completely obliterated, may produce another kind of deceptive relief. This stage is indicated if the only enamel left is found in the deep longitudinal groove-or grooves in the upper cheek teeth-and in the transverse valleys between the cusps. These areas may then be higher than the 
dentine surrounding them (See Plate I, fig. I $a$, R.7032, middle row of cusps of penultimate tooth.)

The loss of anterior cheek teeth has already been discussed. If such loss occurs for instance in the lower dentition, the opposing upper tooth loses its antagonist and from then on its posterior part only is worn if, during the forward excursion of the lower jaw, the succeeding lower cheek tooth comes into contact with it. (See Plate I, fig. I $a$, R.7032, and Plate II, fig. 2, R.7254.)

All lower cheek teeth show pressure wear anteriorly and posteriorly. The same is true for the upper teeth; here, however, little pressure wear is visible anteriorly because the wrinkled anterior surface of the tooth presses against dentine on the posterior face of its predecessor.

\section{THE INCISORS}

(Plate I, figs. 2, 3, Plate II, fig. $4 b$; Text-figs. 33-38)

There are three incisors in both the upper and lower dentitions. Of these six different teeth, four have been found in situ, though the crown is lost in all of them. The alveoli of the incisors are shown on Plates I and II.

The teeth in situ are: $\mathrm{I}_{1}$ in R.7I40, and $\mathrm{I}_{2}$ and $\mathrm{I}_{3}$ in R.7I43; in this specimen only the proximal quarter of the roots is preserved and the specimen is unsuitable for illustration. In the premaxillary $\mathrm{I}^{3}$ has been found in R.7007 and R.7008; here again preservation is such that an illustration of the specimens would serve no purpose.

Both adult forms of Oligokyphus from Windsor Hill have the same incisor formula. Apart from positive evidence for $I_{1}$ there is none available for the incisors of the smallest size-group of Oligokyphus of which only seven dentaries are available (Text-fig. 30, the seven smallest " $G$ "). Apart from the uncertainty of the incisor dentition of the smallest size-group of Oligokyphus, there is thus definite and unambiguous evidence so far as the alveoli of incisors are concerned. As, however, all the available crowns of incisors are isolated, a great amount of fitting is necessary to allot teeth to alveoli.

There are at least thirteen incisor alveoli plus an unknown number-possibly five-for the smallest size-group. Against this number of alveoli there are at present only nine different incisors available, two of which are, however, morphologically identical in both adult forms. It is thus possible to fit eleven alveoli out of a total of thirteen plus. The identification of the following incisor teeth only is based on good factual evidence and is therefore reliable:

Small adult $\mathrm{I}_{1}$. Large adult $I_{1}$. Large adult $I^{1}$. Small adult $\mathrm{I}^{1}$. Large adult $I^{2}$. Large adult $\mathrm{I}^{3}$. Small adult $\mathrm{I}^{3}$.

The identified teeth are described under their respective symbols, the remaining four teeth under the symbols A-D. 
From the foregoing account there is clearly no evidence of " premolars " or of posterior cheek teeth with only one root. Moreover there is no alveolus in the maxilla anterior to the diastema and, therefore, no canines exist in Oligokyphus. All teeth with only one root can therefore safely be described as incisors.
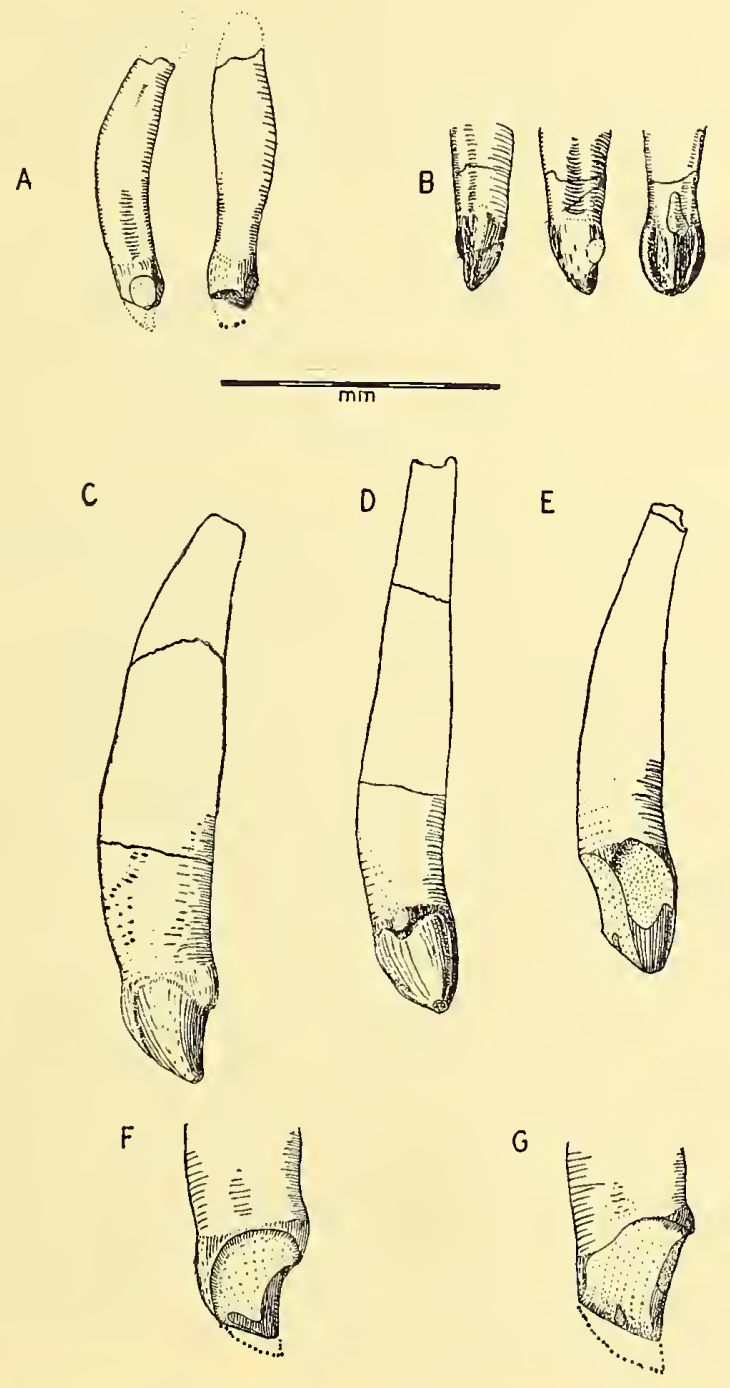

TEXT-FIG. 33-Oligokyphus. The scheme used in the figures of incisors: transversely shaded area-dentine; longitudinally shaded area-enamel; dotted line-demarcation between enamel and dentine ; dotted area-wear facet. A, $\mathrm{I}^{1}, \mathrm{R} .729 \mathrm{I}$, medial and dorsal view. B, $\mathrm{I}^{1}, \mathrm{R} .7290$, lateral medial and ventral view. C, I3 , R.7295, medial view. D, I3 , R.7295, posterior view. E, I ${ }^{3}$, R.7297, posterior view. F, I³, R.7296, medial view. G, I³, R.7297, medial view.

Another preliminary question to be settled concerns the allocation of all the available incisors to the genus Oligokyphus. The possibility that another genus contributed some of the incisors can be dismissed. Apart from dermal ossifications and teeth of fish, no other specimen of a vertebrate has been found at Windsor Hill and the "style" of all the incisors found is such that not one could be excluded from Oligokyphus on morphological grounds; none of the incisors could, for instance, belong to a carnivore. 
In the chapter on biostratonomy, it was shown that the number of specimens of a skeletal element or tooth found is mainly dependent on its size. The number of small specimens is always much less than that of larger elements, irrespective of the original number of specimens of such element occurring in the living individual or, to enlarge the margin of consideration, in the original population of Oligokyphus. It is therefore necessary to rely on the evidence of a few small teeth as much as on the many large teeth. This condition of occurrence makes it probable that a number of small incisors belonging to the dentition of the smallest size-group have not yet been found, but they might occur in a collection two or three times the size of the one now studied.

\section{Description of Incisors}

\section{Description of Incisors of Large and Small Adult Forms : $I^{1}$}

Evidence from the alveolus: Only the proximal part of the alveolus is preserved, being vertically compressed.

Material: R.7290 and R.729I and seven unregistered specimens (Text-fig. 33, A, B.)

No specimen is complete, but the approximate total length of the tooth varies between 5.5 and $9 \mathrm{~mm}$. It is assumed that the tooth is a symphysial $\mathrm{I}^{1}$. The transverse section through the anterior part of the root resembles a right-angled triangle with smoothed corners, the medial and inferior surfaces forming the small sides. Of the incisors this tooth alone has an almost flask-like root, with a marked constriction in its distal third. The posterior part of the root is vertically compressed and grooved. The crown is almost symmetrically spoon-shaped; the concave inferior working surface is limited by two round ridges, no sharp cutting edge being formed on either side. The middle part of the internal ridge is produced into a small prominence. The working surface is longitudinally divided by a slight elevation. With the exception of R.7290 all specimens are strongly worn. The largest facet shears the crown at right angles. This facet is presumably formed by contact with food. A small, shallow and round facet is formed on the medial side of the crown which is due probably to pressure-wear by the identical tooth of the other side of the dentition. There is a long flat facet on the proximal part of the working surface of the tooth (Text-fig. 33, B) which may have been formed by contact with the tip of $\mathrm{I}_{1}$, but this is no more than a tentative suggestion. The variation in size is such that it is likely that both adult size groups are represented in the material.

$I^{2}$

Evidence from the alveolus: The cylindrical and backward curved alveolus in the premaxillary fits exactly the tooth identified as $\mathrm{I}^{2}$.

Material: There are nineteen specimens. R.7292, R.7293 and R.7294 are figured.

Vernier measurements have been taken above the enamel and in the antero-posterior direction. Too much reliance cannot be given to these measurements because no easily identified spot can be located on the tooth and the consistent orientation of all specimens during measurement is impossible. However, though it is unlikely that a repeated series of measurements would give identical figures, it should not produce a reversal of the general result given here. 
Vernier measurements of $\mathrm{I}^{2}$ (I/IOo of mm. estimated).

$\begin{array}{lll}2 \cdot 25 & 2 \cdot 8 & 3 \cdot \text { I5 } \\ 2 \cdot 4 & 2 \cdot 8 & 3 \cdot 2 \\ 2 \cdot 6 & 2 \cdot 85 & 3 \cdot 35 \\ 2 \cdot 6 & 2 \cdot 9 & 3 \cdot 4 \\ 2 \cdot 65 & 3 \cdot I & 3 \cdot 4 \\ 2 \cdot 75 & 3 \cdot I_{5} & \end{array}$

All the specimens appear to belong to the large adult form of Oligokyphus.

Of this material only two specimens are not strongly worn ; R.7292 is actually unworn and is thus the only specimen showing the unobliterated shape of this tooth.

The crown of $\mathrm{I}^{2}$ is short and stout and has only one bent conical cusp. Two cutting edges are developed, the external one being longer and straighter. Between the two edges and on the postero-medial side of the tooth lies a saddle-shaped rounded prominence which is rapidly worn away as the main working surface develops here.

There are three major wear facets. One facet cuts away the tip of the tooth, exposing the pulp cavity in several specimens. This facet was produced by up and down movement of the head, when digging in the ground or when lacerating and scratching hard food or wood. An extensive facet on the internal side of the crown was caused by contact with the external cutting edge of $\mathrm{I}_{1}$; and here emerges one of the numerous difficulties which this material so frequently presents: the wear on the external cutting edge of $I_{1}$ is, if present at all, extraordinarily slight. Identification of the two teeth would have been easier if some specimens of $I_{1}$ had shown more wear externally and the corresponding facets of the two teeth had been more alike. Text-fig. 34, H shows the internal facet merging slightly on to the frontal side of the tooth. It seems therefore that the $I_{1}$, when between the $I^{2}$, had to some extent free play. This was quite possible when the lower jaw was dropped a little and the cheek teeth did not occlude. To this cause may also be attributed the narrow facet which continues the large internal facet posteriorly in R.7294, Text-fig. 34, C. In Text-fig. $34, \mathrm{D}, \mathrm{G}$ is shown a small cup-like facet on the posterior side of the crown; it seems to have been produced by the tip of $\mathrm{I}_{1}$, when this tooth, together with the dentary, was fully retracted. To excavate this facet, the dentary must also have been moved sideways, which would only be possible when the cheek teeth were not in occlusion. So long as the two rows of cheek teeth were in occlusion the movement of the dentary was of necessity exclusively propalinal.

$I^{3}$

Evidence from the alveolus and teeth in situ: the identification of this tooth is proved by the roots of two $\mathrm{I}^{3}$ in situ.

Material: There are forty-seven specimens, of which R.7295, R.7296 and R.7297 are figured (Text-fig. 33, C-G).

The great number of specimens and the great range in size suggest that apart from the two size-groups, which are to be expected in any extensive material of an element of Oligokyphus from Windsor Hill, the material might actually consist of a number of teeth of different position. However, considering carefully the mode of wear and the shape of the crown and the root, the material could not be divided on morphological grounds. It thus seems true that only $\mathrm{I}^{3}$ are concerned. 
The measurement of this tooth has been made in a similar way to that of $\mathrm{I}^{2}$.

$\mathrm{I}^{3}$ is distinctly bicuspid, the small accessory cusp being situated at the upper margin of the crown, internally and on the posterior side of the main cusp. A sharp groove which separates the accessory cusp from the main cusp is continued upwards on both sides of the accessory cusp until the margin of the enamel is reached; from these points the groove descends on both sides to the tip of the main cusp and represents functionally an internal and an external cutting edge. In unworn teeth which are not shown in the figures, the
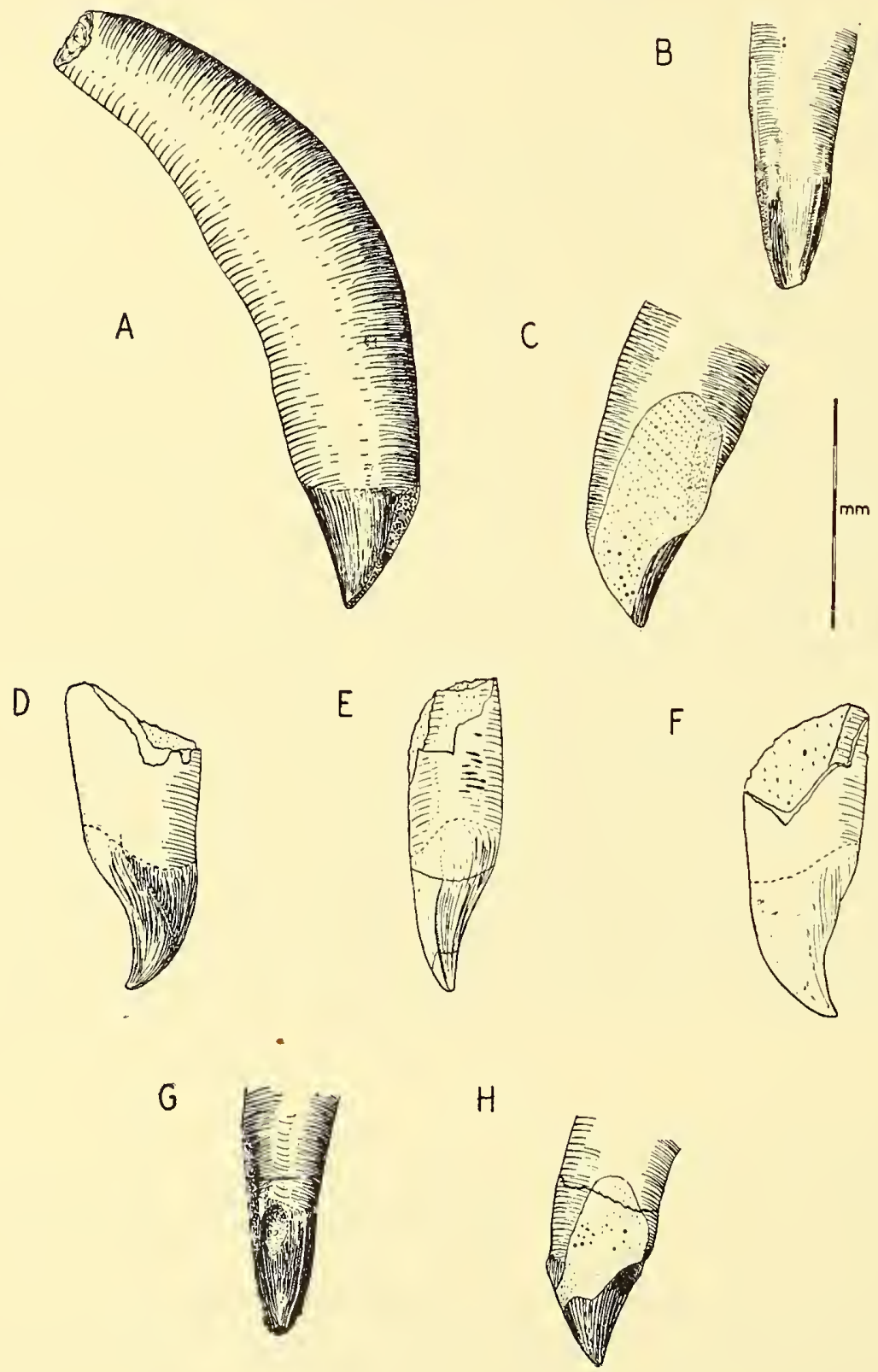

TEXT-FIG. 34.-Oligokyphus. A, Right I², R.7294, lateral view. B, Right $\mathrm{I}^{2}$, R.7294, posterior view. C, Right I ${ }^{2}$, R.7294, medial view. D, Left $\mathrm{I}^{2}$, R.7292, medial view. E, Left I², R.7292, posterior view. F, Left I ${ }^{2}$, R.7292, lateral view ; unworn specimen. G, Right $\mathrm{I}^{2}$, R.7293, posterior view H, Right I², R.7293, medial view. 
point of the accessory cusp is bent backward as in the main cusp. In the figured specimens the very small point which would show this character is already worn off. Many specimens are worn. The most conspicuous wear facets truncate the main cusp; the part of the crown thus lost is indicated by a dotted line in Text-fig. 33, F, G. On the internal side of the crown a large facet develops, which ultimately exceeds the crown proximally ; distally it cuts into the facet which truncates the main cusp. An edge is thus produced between the two facets on which the pulp cavity may be exposed (Text-fig. 33, F, G). On the posterior side of the main cusp a rather shallow facet develops and obliterates the main cusp. All three facets are comparable with those in $\mathrm{I}^{2}$.

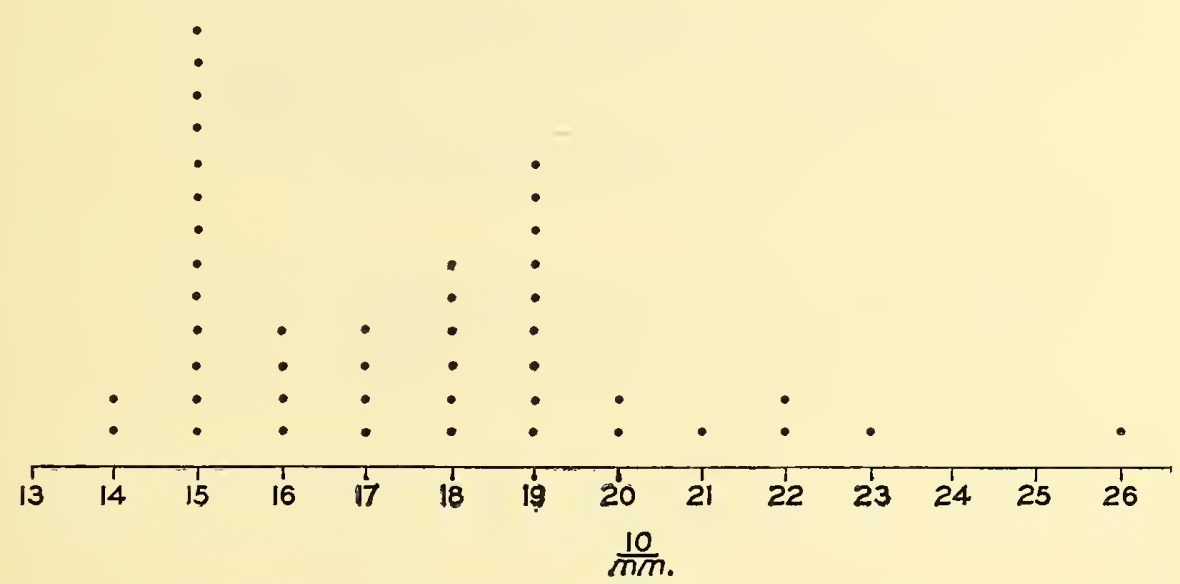

TEXT-FIG. 35- Oligokyphus. Scale showing height of root of $\mathrm{I}^{3}$ above crown in 45 specimens.

$I_{1}$

The large alveolus for this tooth is well preserved in many specimens. Most valuable is R.7I40, in which the distal half of $\mathrm{I}_{1}$ is in situ (Plate II, fig. $4^{b}$ ).

Material: There are more than forty specimens, of which R.7298 and R.7299 are figured.

Only a few specimens show the entire length ; most specimens are either worn and thus lack the point of the crown or part of the root has broken off, or both conditions prevail. The maximum vertical height of the root has been measured.

R.7I40 allows two measurements to be taken, each of which can be compared with a larger number of specimens which permit only one measurement. The specimen is adult and consists of the anterior part of the dentary with the distal half of the diastema and the proximal half of the symphysis in perfect condition. $\mathrm{I}_{1}$ is in situ but only the proximal half of this tooth is preserved.

The height of the horizontal ramus immediately behind the symphysis suggests (Text-fig. 30) that R.7 I40 is either the largest representative of the small adult form or, what is less likely, the smallest representative of the larger adult form. The measurement of maximum height of the root $I_{1}$ (Text-fig. 37) puts R.I740 into the group of small adults but near to the group of large adults and corroborates the evidence from the measurement of the height of the dentary. Furthermore $\mathrm{I}_{1}$ is almost certainly identical in the large and in the small form of Oligokyphus. $\mathrm{I}_{1}$ is procumbent. The figures of $\mathrm{I}_{1}$ (Text-fig. 36, A-E) need few supplementary remarks. The enamel is extraordinarily thin all over the crown. The line limiting 
the extent of the enamel is almost imperceptible. The twisting of the tip of the crown to the internal side, bringing the external cutting edge slightly above the internal cutting edge, is the character on which most easily a right tooth can be distinguished from a left. But this character is discernible only in a frontal view of the tooth (Text-fig. $36, \mathrm{E}$ ). The external side of the root is slightly convex, the whole internal side is flat, though a very slight concavity is perceptible along the proximal three-quarters. Many crowns show very little wear. A minute facet along the external cutting edge is to be seen at least on all specimens which are not newly formed. The worn tip of the crown is smoothly truncated and a flat facet is produced on the working surface.

A

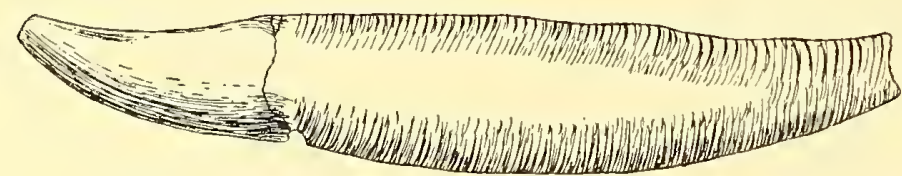

B

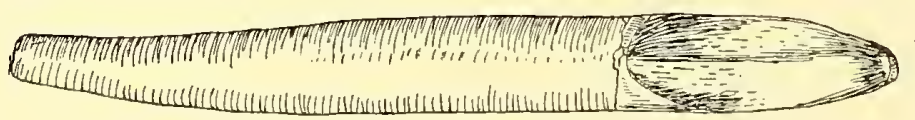

c

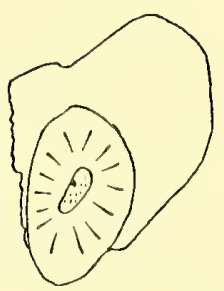

D

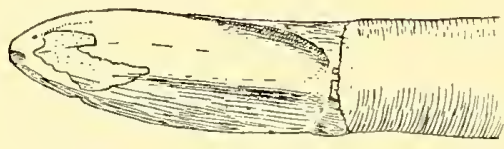

E

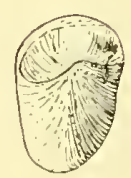

TexT-FIG. 36.-Oligokyphus. A, Left, $\mathrm{I}_{1}$, R.7298, lateral view. B, Left $\mathrm{I}_{1}$, R.7298, dorsal view. c, Root of left $I_{1}$ in situ, R.7I40, frontal view. D, Left $I_{1}$, R.7299, dorsal view; a large specimen. E, Left $\mathrm{I}_{1}, \mathrm{R} .7299$, crown view. Scale in $\mathrm{mm}$.

The absence of extensive wear in many specimens of the large sample of $I_{1}$ is the more astonishing when compared with its opponent $\mathrm{I}^{2}$, which shows extreme wear in most specimens. This disparity of wear is hardly due to sampling errors, though there are only nineteen $I^{2}$ and more than forty $I_{1}$. The lack of extensive wear on $I_{1}$ may be explained by the precarious jaw articulation of Oligokyphus, which did not allow a robust use of the tooth. $I_{2}$ and $I_{3}$

The proximal fifth of the roots of both $I_{2}$ and $I_{3}$ are in situ in the large adult specimen R.7I43. Both roots are flattened antero-posteriorly. No isolated tooth seems likely to be either $\mathrm{I}_{2}$ or $\mathrm{I}_{3}$. Evidence from alveoli in small adult specimens shows that these two teeth also occur (Plate II, figs. $4 a, b)$.

There are various incisors whose position in any dentition of Oligokyphus is uncertain. All are considerably smaller than those previously described and apart from one-tooth A-all are rare. Most types, however, occur more than once and where this is the case identity 
of form can be established, which makes it certain that the teeth are not abnormal. Probably all these teeth belong to the smallest size-group of Oligokyphus which is represented in the osteological material only by the seven smallest dentaries. (See the seven smallest "G's" in Text-fig. 23). The teeth are lettered A-D for convenience of reference.

$A$. Seventeen specimens, of which R.7300 and R.730r are figured. A typical specimen is $9 \mathrm{~mm}$. long, the height of the tooth (as for $I_{1}$ ) is $2 \mathrm{~mm}$., the length of the crown is $2.5 \mathrm{~mm}$. The similarity of $A$ and $I_{1}$ is striking. Characters of both teeth differ only in degree. In the crown the concavity of the working surface is deeper, the convexity of the inferior surface more distinct. The crown is more twisted to the internal side, the edge of the tip being inclined as much as $45^{\circ}$ (Text-fig. $3 \mathrm{r}, \mathrm{B}$ ). In transverse section the root is pearshaped, as shallow longitudinal concavities are developed on each side. The external and internal sides of the root cannot be distinguished. As in $\mathrm{I}_{1}$ a slight medial prominence runs
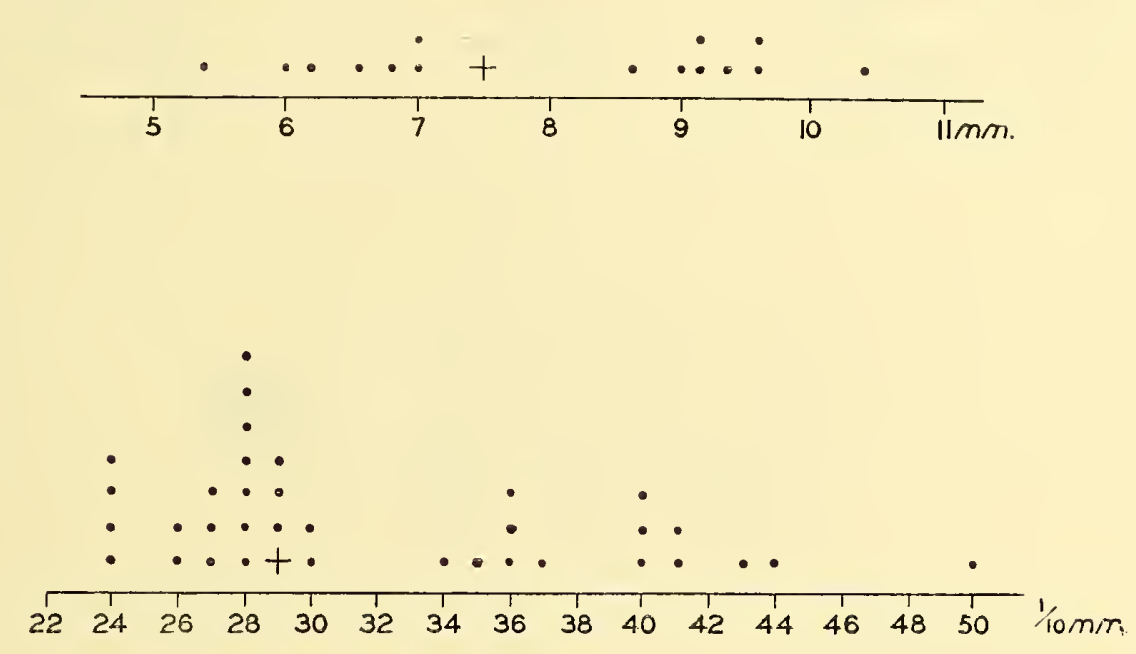

TEXT-FIG. 37.-Upper diagram: Height of dentary behind symphysis. Measurable specimens, $+=$ R.7I40. Lower diagram: Maximum thickness of $\mathrm{I}_{1}$. Measurable specimens, $+=\mathrm{I}_{1}$ in R.7I40.

along the whole concave working surface of the crown. The kind of wear found in $\mathrm{I}_{1}$ also occurs in A.

On this evidence it seems most probable that this $I_{1}$ belongs to the form to which the five smallest dentaries also belong-discussed with the lower dentition.

$B$. A single specimen, R.7302, of which only the distal half is preserved ; the approximate total length of this tooth was $8 \mathrm{~mm}$. The root is almost cylindrical, not compressed laterally as in A. The crown is characterized by a sharp edge running longitudinally along the working surface of the crown, thus dividing this surface into two sub-equal and sloping halves. The internal and external cutting edges, which separate the working surface from the inferior convex face, are situated so far down as to restrict considerably the latter surface. This face is therefore much smaller than in either $I_{1}$ or $A$. The presumed internal half of the working surface is slightly larger than the external one. In frontal view B is broader than it is high, the circumference is roughly hexagonal and not similar to the crown view of A. At the proximal margin of the concave working surface, four minute cusps are to be seen. The specimen is unworn. 
C. There are five specimens, of which R.7303 is figured. The tooth is similar to $\mathrm{I}_{1}$, $\mathrm{A}$ and $\mathrm{B}$. The material includes the smallest tooth found at Windsor Hill, the height of its crown measuring only $0.6 \mathrm{~mm}$. In another specimen the same distance is $\mathrm{I} \cdot 4 \mathrm{~mm}$. The tooth is unicuspid, bluntly pointed, and straight. The root is slightly compressed. The working surface is very extensive ; in the region of the tip of the crown it claims threequarters of the circumference of the crown. The external cutting edge is placed as in $\mathrm{I}_{1}$ and A, but the internal cutting edge, from the tip of the crown backwards, falls down very steeply, and, by ultimately reaching again the upper side of the tooth, includes half of the internal

A

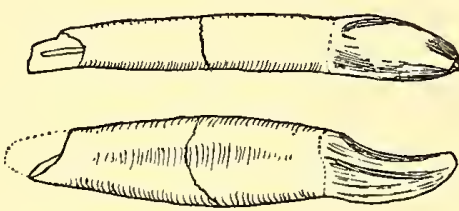

B

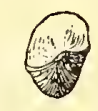

D
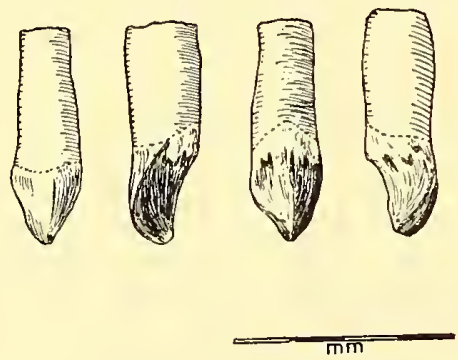

$\mathrm{E}$

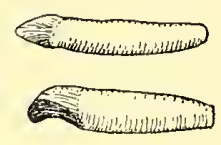

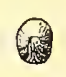

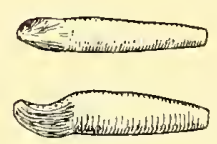

C

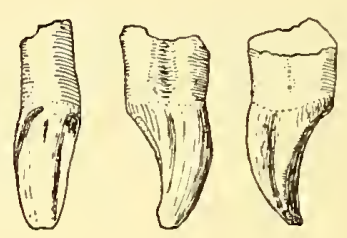

TEXT-FIG. 38.-Oligokyphus. Incisors of unknown position. A, Tooth " A" right, R.730I, dorsal and lateral view. B, Left tooth " A," R.7300, crown view. c, Left tooth " A," R.7300, dorsal, lateral and medial view. D, Tooth "B," R.7302, four views and crown view. E, Left tooth "C", R.7303, ventral and medial view ; crown view ; dorsal and lateral view. F, Tooth " D," R.7304, four views and crown view. Scale in $\mathrm{mm}$.

side of the crown into the working surface. Text-fig. 38, E (cf. also 38, B of A, R.7300) illustrates this state of affairs. The twisting of the point of the crown towards the internal side is therefore much stronger than in A. If worn points of the crown were available, the apical facet would stand almost vertically.

$D$. Three specimens, of which R.7304 is figured above. This tooth is completely preserved and measures $3 \mathrm{~mm}$. in length. In transverse section the root is elliptical, the long axis running interno-externally. The crown is bicuspid, a small accessory cusp being present at the base of the working surface. The working surface is saddle-shaped, slightly concave 
longitudinally and convex transversely. The accessory cusp is separated from the main cusp by a tiny notch which is not connected either with the internal or the external cutting edges. The point of the accessory cusp is situated towards the internal side.

\section{FUNCTION OF THE DENTITION}

In considering the function of the dentition of Oligokyphus, it is far more difficult to interpret the function of the incisors than of the cheek teeth. The unbroken battery of

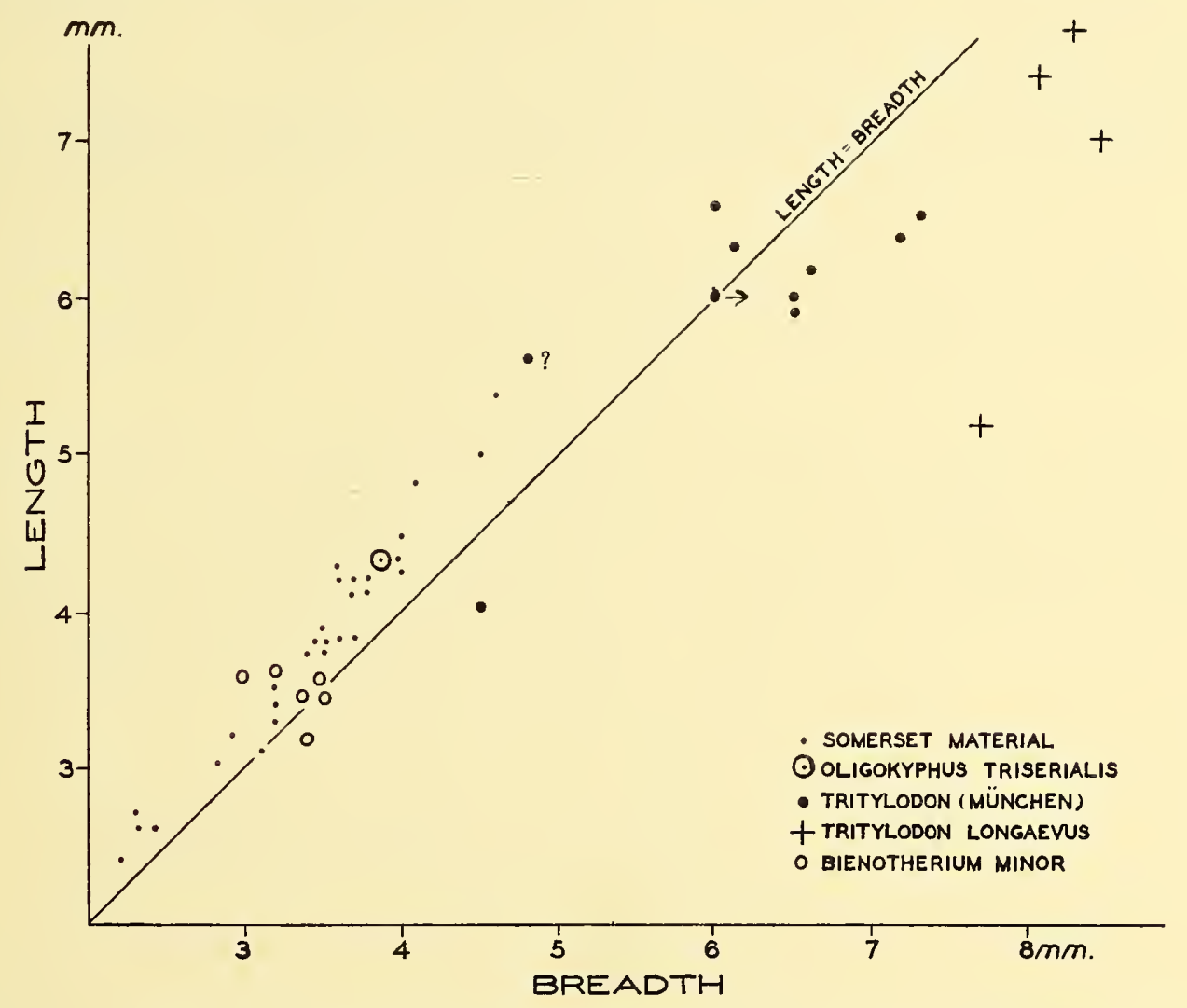

TEXT-FIG. 39--Measurements of length and breadth of isolated upper Tritylodontid cheek teeth. The Somerset material includes teeth from all positions of all three size-groups, but the ultimate tooth is excluded. To the actual measurements of the associated teeth in Tritylodon and Bienotherium that amount of the length which is hidden, due to imbrication of the teeth, has been added.

similar "molariform " cheek teeth, working as a functional unit, is an effective triturating and comminuting apparatus which indicates a vegetarian diet. The crescent faces of the cusps of the cheek teeth, facing in opposite directions in both jaws and being moved along each other, are well fitted to cut long fibres into small pieces in one stroke. This effect is lost by wear and the animal was past its prime when the surfaces of the crowns of the cheek teeth were differentiated merely into shallow longitudinal ridges and all transverse elements of the crown were worn off.

The wide diastema between the incisors and the cheek teeth effects division of labour, the incisors being exclusively food-gatherers, the cheek teeth being exclusively triturators. 
The diastema also indicates the presence of a tongue well developed into a grasping organ and taking an integral part in the seizing and comminuting of the food.

The cheek teeth functioned with the jaw almost closed. The maximum gape was small and the cheeks always hid the cheek teeth. As the cheek teeth functioned only during propalinal motion of the jaw, they were accommodated behind the ascending ramus of the dentary, thus approaching the fulcrum of the jaw and assuming the most favourable position mechanically.

Comparing the incisors of Oligokyphus with those of modern herbivores, it is noteworthy that their " diprotodont" arrangement is neither comparable with that of rodents nor with the cropping apparatus of artiodactyls. A dentition adapted for the utilization of grass as food is ruled out for Lower Liassic time.

The arrangement of the incisors of Oligokyphus is vaguely comparable with that of Erinaceus, Hippopotamus and Moeritherium; they form a grasping and seizing apparatus. 


\section{TAXONOMY}

It is desirable to discuss the taxonomy of Oligokyphus before the post-cranial skeleton has been dealt with, because of the following facts: The genoholotype of Oligokyphus is one single tooth. Of the Oligokyphus material from Windsor Hill dentitions and teeth are more numerous than other parts of the skeleton and have been the only suitable material to demonstrate that more than one size-group is present. Furthermore, as teeth do not grow, their evidence in respect to specific distinction would be preferred to post-cranial elements of the skeleton, growing probably during the whole life of the animals concerned.

So far no reference has been made to-a species of Oligokyphus.

The discussion of the taxonomy of the material is a necessity which is undertaken reluctantly. That the genus under discussion is Oligokyphus there can be no doubt. That this genus differs in the proportions of its upper cheek teeth from Tritylodon is also clear. That the family Tritylodontidae is an extremely narrow and well-defined family no one will contest.

The main taxonomic question at present is the relation of the Tübingen materialOligokyphus triserialis and Oligokyphus biserialis - to the new Somerset material. On the one hand there are two waterworn and isolated cheek teeth, on the other there are more than 2000 specimens. Apart from this disparity, it is found that Hennig's measurements contradict his figures and the facts.

Hennig's measurements are as follows (Hennig I922: 206, 225) :

$\begin{array}{lcc} & \text { Length } & \text { Breadth } \\ \text { Oligokyphus triserialis } & 4.0 \mathrm{~mm} . & 4.0 \mathrm{~mm} . \\ \text { Oligokyphus biserialis } & 3.5 \mathrm{~mm} . & 2.5 \mathrm{~mm} .\end{array}$

The measurements taken from Hennig's figures (Hennig I92I, pl. 2, fig. II $b$, pl. 3, fig. $5^{b}$ ) are as follows :
O. triserialis
Length
Breadth
O. biserialis
$4 \cdot 4 \mathrm{~mm}$.
$3.6 \mathrm{~mm}$.
$4.5 \mathrm{~mm}$.
$2.5 \mathrm{~mm}$.

Through the courtesy of Prof. Schindewolf accurate measurements made by Dr. R. Dehm have now been received, as follows :
O. triserialis
Length
Breadth
$O$. biserialis
$4 \cdot 3 \mathrm{~mm}$.
$3.9 \mathrm{~mm}$.
$3.6 \mathrm{~mm}$.
$2 \cdot 2 \mathrm{~mm}$.

These measurements have been used in Text-figs. 39 and 40 and show that there are no differences in the proportions and dimensions of large teeth from Somerset and the two specimens from Tübingen. 
The cusp pattern of Oligokyphus triserialis (see p. 73) is even less reliable than are Hennig's measurements, because the tooth is waterworn and its present condition would have to be taken into account only if more cusps were present than there are in the Somerset material. But this is not the case.

There remains the question of genera (Text-figs. 39, 40). Whereas Oligokyphus triserialis does not differ in size or in proportion from the Somerset material, Tritylodon differs both in size and in proportion. The cheek teeth of Tritylodon are considerably broader than the teeth from Somerset. Actually there is only one tooth in the Tritylodon material in Munich which has the same proportions as the Somerset material. The general size of the upper cheek teeth of Tritylodon is about twice that of the large adult tooth from Somerset.

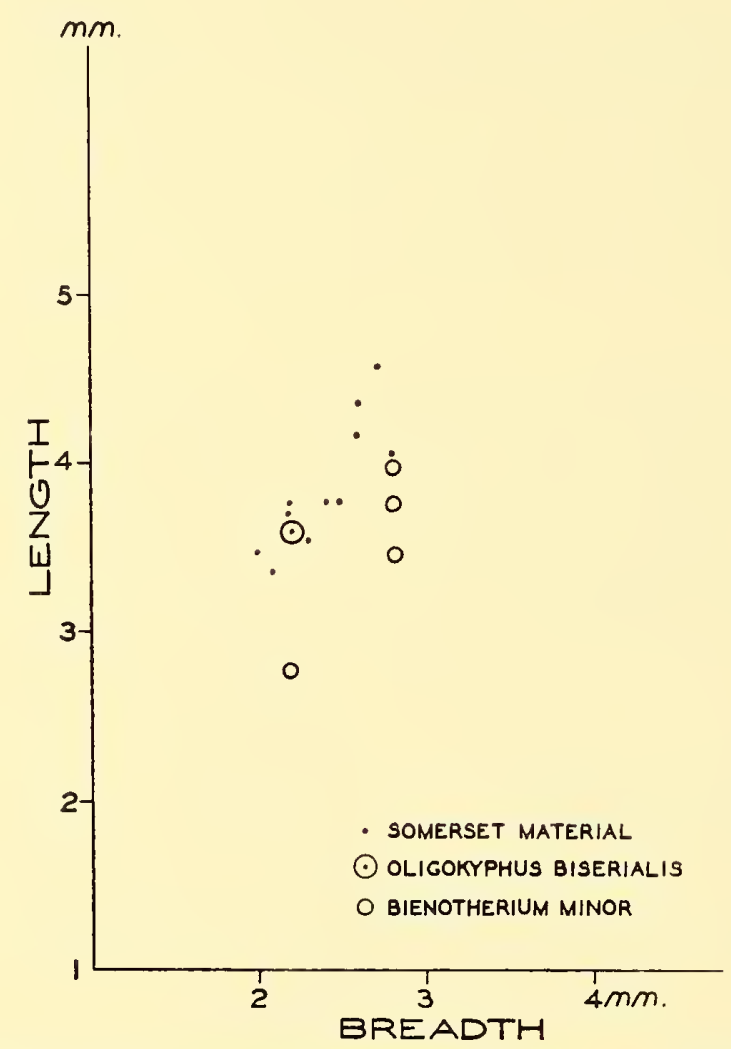

TEXT-FIG. 40.-Length and breadth measurements of lower Tritylodontid cheek teeth.

In conclusion: the large Somerset animal compares well with Oligokyphus triserialis. The genus Oligokyphus has upper cheek teeth about half as long and broad as Tritylodon. The upper cheek teeth of Tritylodon are generally broader than long, the upper cheek teeth of Oligokyphus are longer than broad.

One word more concerning $O$. biserialis. This is not a species; not even in a taxonomic sense, unless one disregards the explicit and well-expressed intention of Hennig. He writes (Hennig I922: 23I): "Mit voller Absicht habe ich als Beiname (Hennig does not write "Species Name") in beiden Fällen eine Bezeichnung gewählt, die den sicher erkennbaren Unterschied einstweilen behelfsmässig widergibt. Es ist klar, das bei etwa als notwendig erkannter spezifischer Vereinigung keiner von beiden beibehalten werden kann ". (I am afraid 
that in this instance it is not permissible to conform to Hennig's wish, the genoholotype is Oligokyphus triserialis). Hennig continues: "Ich wünsche damit klar zum Ausdruck zu bringen, dass eine solche binomenklatorische Benennung nicht den Wert einer zoologischen Artunterscheidung beanspruchen darf . . ." As O. biserialis is thus not a species it is also not a holotype. It is a specimen which has two names, the second being biserialis. This name is of the same kind and status as are the "names" of some of the incisors of the Somerset material, which are A, B, C and D.

As for Bienotherium, B. yunnanense and B. elegans have upper cheek teeth broader than long and they are about twice the size of the large adult Oligokyphus. The proportions of upper and lower cheek teeth of the three specimens of Bienotherium minor are those of a medium sized Oligokyphus. The cusp pattern of these three specimens is unknown. Even if it were known and identity with that of Oligokyphus could be proved, the genus Oligokyphus would not have a greater biozone as has been stated. The occurrence of the genus Oligokyphus in Europe and in South-East Asia would emphasize the world-wide range of the Tritylodontidae.

It is clear that there is no generic distinction between the Somerset material and the Tübingen teeth. This is acknowledged by giving the Somerset material the generic name Oligokyphus. As for the species the most satisfactory procedure, for the time being, is to give the two animals from Somerset new specific names. The reasons for this procedure are as follows:

(I) The Tübingen teeth are from the Upper Rhaetic bone bed; the Somerset material is Charmouthian.

(2) Apart from their dimensions, the Tübingen teeth do not show any other reliable and diagnostic character.

(3) The Tübingen teeth being isolated, it is impossible to be certain about the dentitions to which they belong.

(4) To give the Somerset material the specific name $O$. triserialis would imply identity of the two finds, which cannot be proved.

If well-preserved dentitions turn up in the Rhaetic, it will be time to re-discuss this matter. The histograms of measurements of different teeth, and especially the graph dealing with the breadth and height of the horizontal ramus of the dentary (Text-fig. 30) show that the distribution in size of many elements is bimodal. If such bimodality occurred only in one or a few elements of the total, it would most probably be due to a sampling error. But it is significant that the bimodality is a consistent character of most elements measured. Does that mean that at Windsor Hill there are two species of Oligokyphus? The difference in linear dimensions of the two adult forms is about one-third, but their difference in weight is more than Ioo per cent. Thus there is really a significant disparity in size. Furthermore the coefficient of variation for the dentaries (height of the horizontal ramus at the anterior end of the masseteric fossa) is $26 \cdot 5$, a coefficient quite incompatible with monospecific and non-polymorphic material. The coefficient of variation of the two adult forms only would naturally be smaller, and a high coefficient is to be expected if a species is sexually dimorphic and both sexes are considered together. If the two adult forms of Oligokyphus had come from different quarries or at least from different fissures in the same quarry, one might have regarded them as two different species, but this is not the case. The place of deposition is identical for all size-groups and the same applies to 
the thanatocoenosis. It can be assumed with good reason that the same predator preyed on a population of Oligokyphus of which we have found the bones.

The two adult forms of Oligokyphus may not have occupied the same ecological niche on their Mendip island, but the carnivore may quite easily have preyed on both. Even if the two adult forms of Oligokyphus are different sexes of the same species, it does not follow that the two sexes occupied the same ecological niche during the whole of a year. Until a method is found which enables one to sex fossil bones, there can be no certainty in claiming sexual dimorphism in a fossil population. In any case little is known about sexual dimorphism in respect of Lower Liassic mammal-like reptiles.

The large adult form from Windsor Hill is, therefore, named Oligokyphus major and the small adult form Oligokyphus minor, with the maxillaries R.7030 and R.7025 as the respective holotypes (Plate II, fig. I; Text-fig. 26). It is possible that the two adult forms are different sexes but there is no indication as to which might be male and which female.

The five smallest dentaries are not given specific rank. In comparison with the two adult forms, too little is known about this group.

When describing the dentition of a mammal it is customary to give a tooth formula. It has been made clear that the method of change of the dentition in Oligokyphus is of a kind hardly comparable with that of any other animal and that a tooth formula is not a good means of expressing it. Unerupted or functionless teeth posteriorly, and an unknown number of lost teeth anteriorly, make it hardly possible to offer a tooth formula applicable to at least the majority of specimens discovered in the future. As given here, unerupted and functionless teeth are counted as well as the "zero" tooth in the lower jaw.

\begin{tabular}{|c|c|c|}
\hline Oligokyphus major. & $\mathrm{I}^{1-3}$ & C.T. ${ }^{1-7}$ \\
\hline & $\mathrm{I}_{1-3}$ & C. $T_{\cdot 1-7}$ \\
\hline Oligokyphus minor. & $\mathrm{I}^{1-3}$ & C.T. ${ }^{1-6}$ \\
\hline & $I_{1-3}$ & C. $T_{\cdot 1-6}$ \\
\hline
\end{tabular}

Atlas

THE VERTEBRAL COLUMN

Material: Atlantal neural arch: R.73I0-I2 and seven fragments (Text-fig. 42, A-C). Intercentrum : R.73I3a, belonging to O. major (Text-fig. 42, D), R.73I3b, O. minor.

For comparison with Oligokyphus, a neural arch of Diademodon sp. (R.202 in Prof. D. M. S. Watson's collection) is shown in Text-fig. 4I.

The posterior process of the neural arch as well as the pro-atlantal articulation seen in Diademodon are absent from Oligokyphus. Thus, the criteria for anterior and posterior are missing, and it is difficult to orientate the specimens.

In both Diademodon and Oligokyphus the atlantal neural arch has two portions, a bi-faceted part, which is solid, and a plate-like dorsal part, which is produced ventrally as the atlantal pleurapophysis. On one side the dorsal plate is detached from the facet, producing a deep groove between them. On the other side the margin of the dorsal plate and that of the facet are confluent. If the first and second spinal nerves of Oligokyphus 
follow the same course as in Diademodon and many mammals, the first would run in the deep groove and the second would run lateral to the facet which is confluent with the dorsal plate. The (anterior) canal of Diademodon would be represented in Oligokyphus by a deep groove, and the (posterior) groove of Diademodon would be represented in Oligokyphus by a
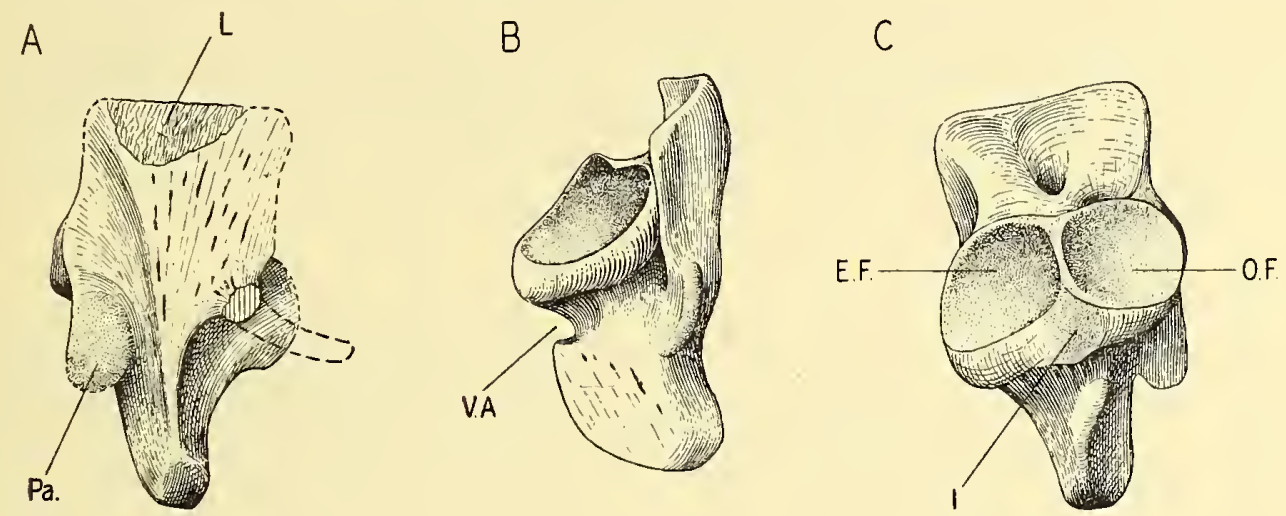

TEXT-FIG. 4I.-Diademodon sp. Left atlantal arch, R.202 in Prof. D. M. S. Watson's collection. Not to scale. A, Lateral view. B, Anterior view. C, Medial view. $E F$, facet for epistropheus ; I, articular area for atlantal intercentrum ; $L$, insertion area for ligament keeping the two halves of the atlantal arch together; $O F$, facet for exoccipital ; $P a$, articular facet for proatlas; $V A$, notch for vertebral artery.

B

A
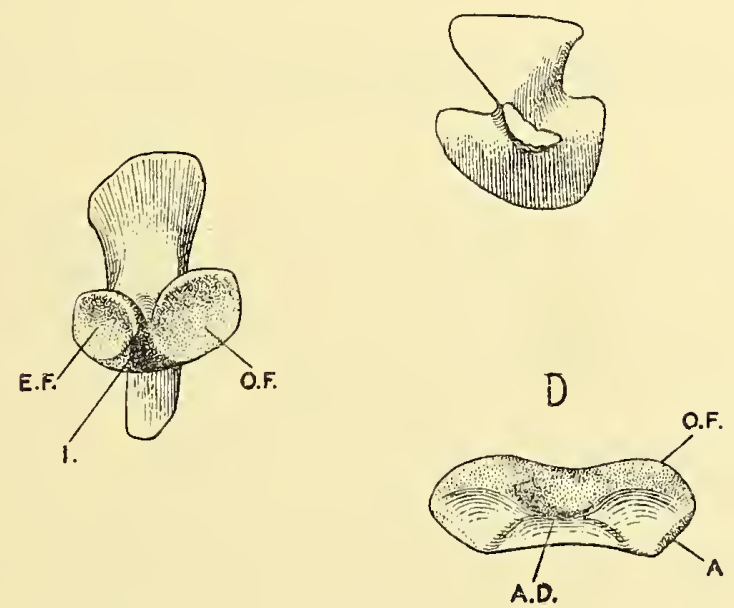

C

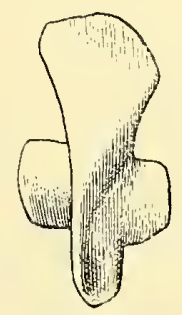

TExT-Fig. 42.-Oligokyphus. A-C, Left antlantal arch, R.73Io. $\times 4$. A, Medial view. B, Ventral view. C, Lateral view. D, Oligokyphus major. Atlantal intercentrum, R.73I3. A, Articular area with atlantal arch. $A D$, articular area for dens epistrophei ; $E F$, facet for epistropheus. $I$, articular area for atlantal intercentrum; $O F$, articular area for occipital condyle.

confluent face. No co-ossification takes place between the three elements composing the atlas and no foramina of any kind are found in the neural arch. Three specimens retain the atlantal pleurapophysis but the distal end is broken in all, indicating that it was a rather long process. The occipital facet is bigger and more concave than the axial one, which accords with the articular surfaces of these bones. Ventral to the junction of the two 
facets there is an articular area for the lateral end of the intercentrum. The atlantal neural arch is featureless, probably because Oligokyphus is a much smaller animal then Diademodon.

The sagittal section of the intercentrum shows that this bone is thick anteriorly and thin posteriorly. The basioccipital and part of the exoccipitals articulate with the anterior margin of the dorsal surface, although the basioccipital does not contact the central part of this surface. Both the infero-posterior margin of the basioccipital and the supero-anterior margin of the intercentrum are slightly concave. The middle part of the intercentrum also has an articular surface, which may articulate with the surface behind and below the dens of the axis.

Axis (Text-fig. 43, A-C)

Broili \& Schröder (I936:62) have described the axis of an advanced Cynodont. The three elements composing the centrum of this bone are clearly visible, namely the atlantal pleurocentrum, and the intercentrum and pleurocentrum of the second vertebra. The

A

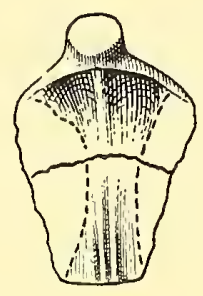

$B$

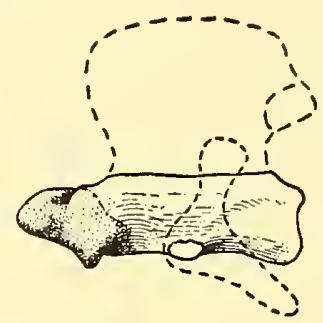

C

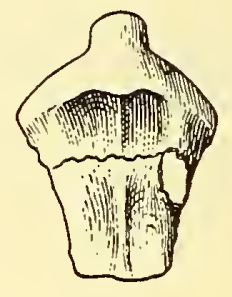

TEXT-FIG. 43.-Oligokyphus minor. Body of epistropheus, R.73I5. $\times 4$. A, Dorsal view. $B$, Lateral view. C, Ventral view.

condition in Diademodon appears to differ in that the atlantal articulation is distinctly trilobed, though this may be due to better preservation. This trilobed condition is strikingly similar to that in Ormithorhynchus. In Diademodon there is, however, still no trace of a dens, the thin and elongated extremity of the axis. Until now this has only been known in mammals, and it is derived from the proatlantal pleurocentrum. There are three specimens of the axis: from Oligokyphus major (R.73I4) and from O. minor (R.73I5-6); R.73I4 alone contains anything of the neural arch. An axial intercentrum is not visible on any of the specimens, but a suture between the "odontoid ", viz. the atlantal pleurocentrum, and the succeeding vertebra is distinct. For the first time the dens of the axis, well preserved in both specimens of $O$. minor, can be recorded in a Therapsid. No suture is visible between the dens and the "odontoid". The anterior face of the axis of Oligokyphus bears a striking similarity to that in Tachyglossus. Below are listed the main characters of the atlas-axis complex in Diademodon, Oligokyphus and in mammals.

Articulation for proatlantal arch on atlantal arch Present Posterior process of atlantal arch Unity of three atlantal elements in the adult Dens of axis
Absent

",

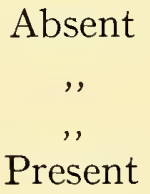

Absent

Present 


\section{POST-AXIAL VERTEBRAL COLUMN}

The vertebral material between the axis and the sacrum is not sufficiently complete for the determination of the exact position in the column of all specimens. The vertebrae therefore will be described as derived from successive regions.

The inferior margin of the anterior or the posterior face of the presacral vertebrae is not bevelled to such a degree as to indicate the presence of intercentra, and the absence from the material of intercentra, apart from the two atlantal ones which have been mentioned, corroborates this. Many small elements have been found in the matrix, including chevron bones and claws, and the presacral intercentra would have been preserved if originally present.

Cervical vertebrae (Text-fig. $44, \mathrm{~A}-\mathrm{C}$ )

Material: R.73I7-R.7321; third to seventh vertebrae.

A typical vertebra bears a short spinous process. The centrum is slightly broader than long and has a slender neural arch. The pre- and postzygapophyses are well developed. The postzygapophyseal facet faces outward and downward, preventing rotation. All the cervical ribs are broken near their attachment, their sutures with the centrum being obliterated in all (3) specimens. There is a wide vertebrarterial foramen. The tubercular attachment to the diapophysis is posterior, the capitular attachment to the parapophysis is anterior. The centra of the cervicals are slightly procoelous; both anterior and posterior surfaces of each vertebra however are dented in the middle, betraying the small role the notochord plays in the adult. The centrum has a midventral keel.

As all presacral vertebrae carry ribs, it is impossible to designate, in the strict sense, lumbar vertebrae. It is, however, possible to place the vertebrae in one of six regions. Each of these regions is described below beginning at the anterior end.

\section{Region I}

Material: R.7325, R.7328. Eighth vertebra (Text-fig. 44, D-E)

The vertebra is broader than long. The spinous process is not very long and is tilted backward. The dorsal edge of the spinous process makes an angle of $45^{\circ}$ with the axis of the vertebra. Pre- and postzygapophyses are well developed, the postzygapophyseal facet faces outward and downward as in the cervicals. The tuberculum articulates with the whole lateral surface of the diapophysis, the capitulum with a parapophysis, which is situated at the anterior margin of the centrum ; most of the capitulum articulates here, and no parapophyseal facet is developed at the posterior margin of the centrum. There is a wide vertebrarterial foramen. The anterior and posterior faces of the centrum are similar to those in cervicals. There is a smooth midventral keel on the centrum, giving it a triangular outline in transverse section.

\section{Region II}

Material: R.7326. Ninth vertebra.

The left side of R.7326 is identical with vertebrae of the first region, but its right side (Text-fig. 44, F) differs in the following points: The tubercular facet is well separated from 
the diapophysis. The vertebrarterial foramen is narrow. There is no midventral keel on the centrum. The vertebra is platycoelous (opisthocoelous).

\section{Region III}

Material: R.7322-24, R.7327, R.7329. These belong to the region from the tenth to fifteenth vertebra (Text-fig. $45, \mathrm{~A})$.

The centrum is a little longer than broad. The spinous process extends beyond the postzygapophysis and its anterior edge makes an angle of $35^{\circ}$ with the axis of the vertebra. Pre- and postzygapophyses are weak. The postzygapophyseal facet faces downward and

A

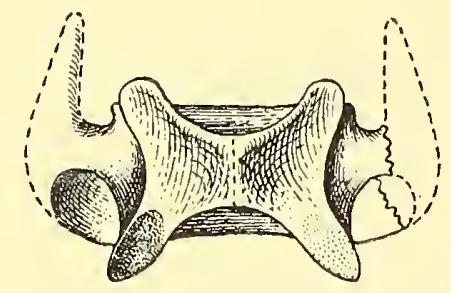

D

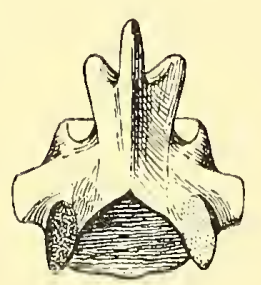

B

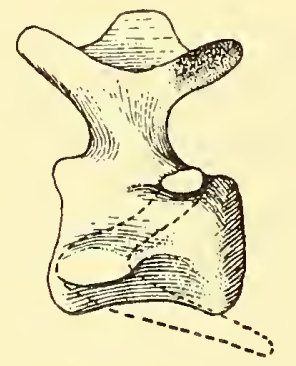

C

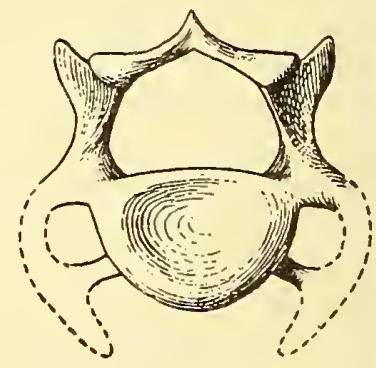

$E$

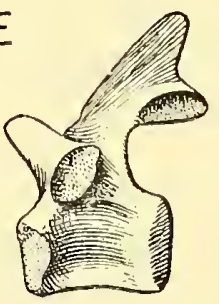

F

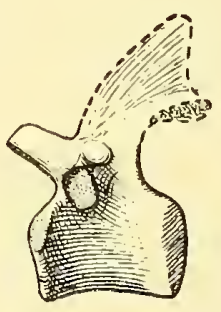

TEXT-FIG. 44--Oligokyphus. Cervical and thoracic vertebrae. $\times 4 . \mathrm{A}-\mathrm{C}$, Cervical vertebra based on R.7320 and 732I. A, Dorsal view. B, Lateral view. C, Anterior view. D and E, Eighth vertebra, R.7328 (Region I), in dorsal and lateral view. F, Ninth vertebra R.7326 (Region II), in lateral view.

very slightly inward, thus allowing rotation of adjacent vertebrae. There is a rather strong diapophysis, which is well separated from the articulation of the rib. Though capitular and tubercular facets are still distinct, there is no vertebrarterial foramen. The centrum is well rounded on the ventral side. The centra of this region are platycoelous.

\section{Region IV}

Material: R.7330. Sixteenth vertebra (Text-fig. 45, B).

R. 7330 differs from vertebrae of the third region in the following points: The spinous process is a little shorter, the small zygapophyseal facets are horizontal. The diapophysis is situated in the middle of the vertebra and is shorter. The rib articulating with this vertebra 


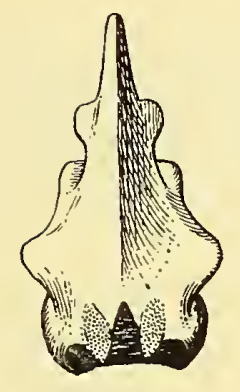

A
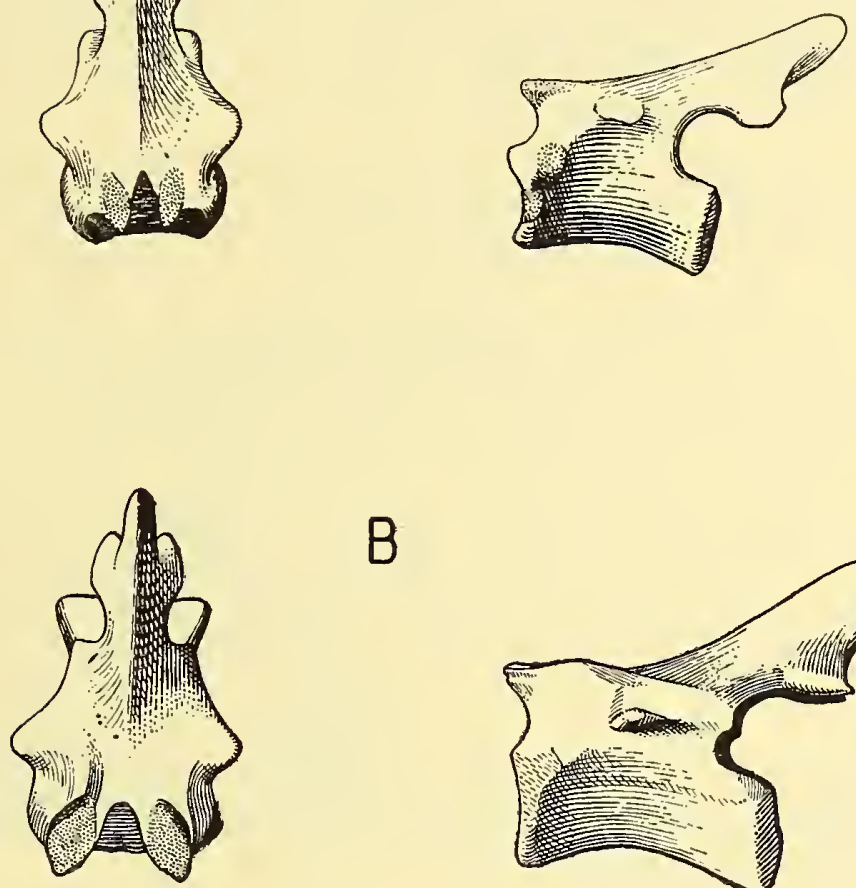

B
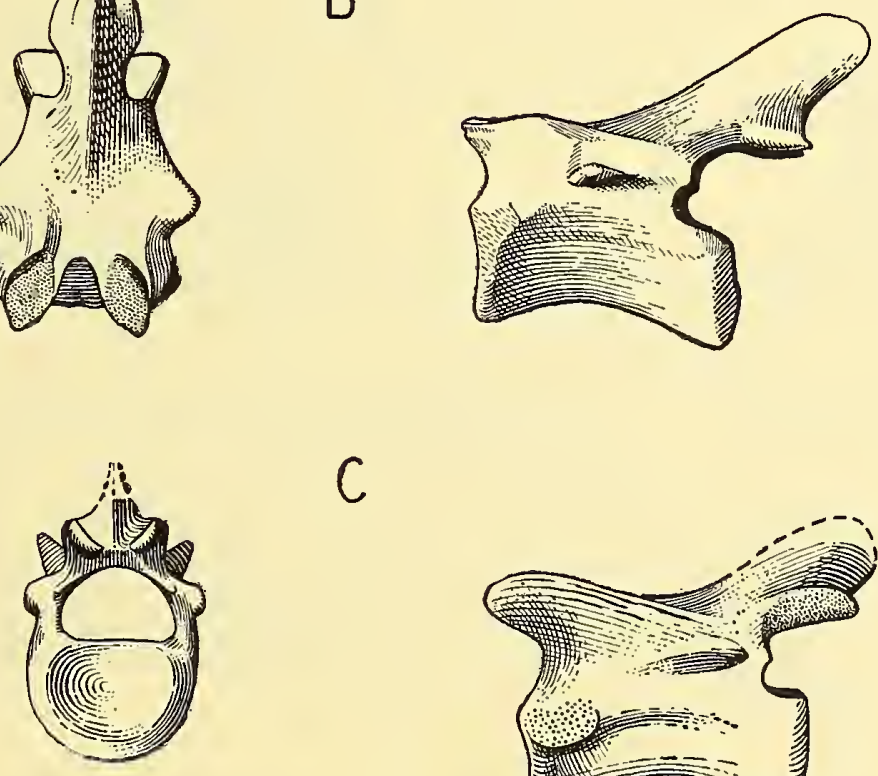

C
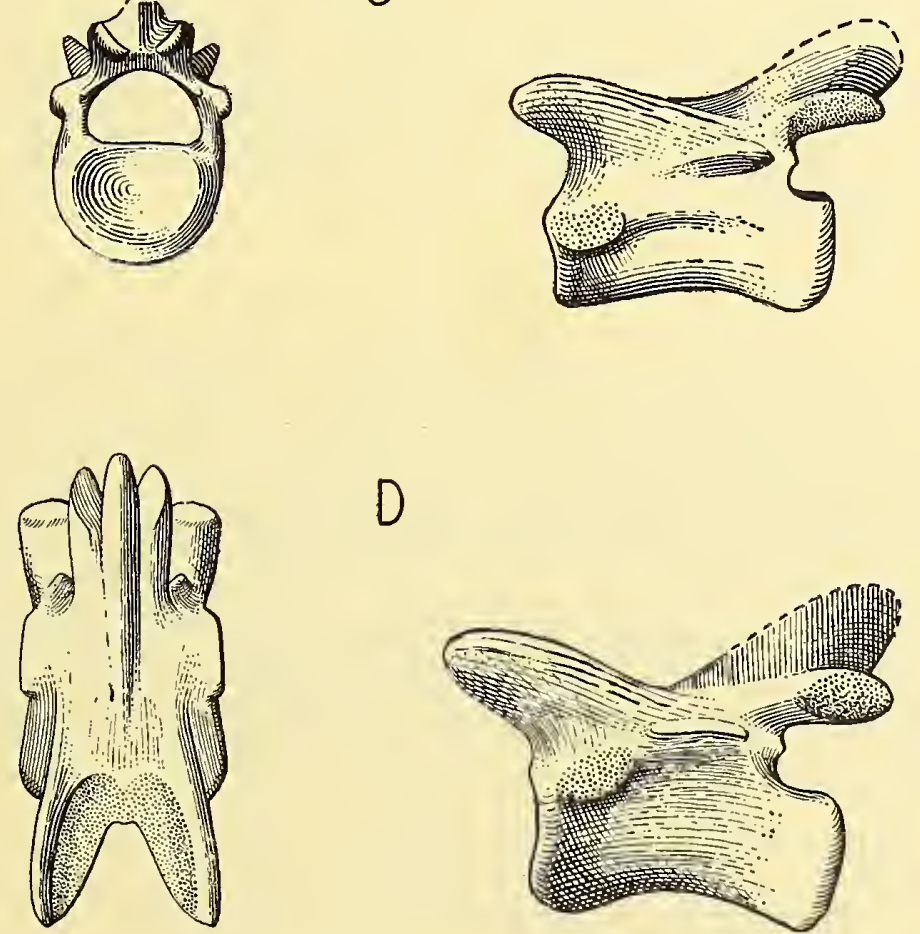

D

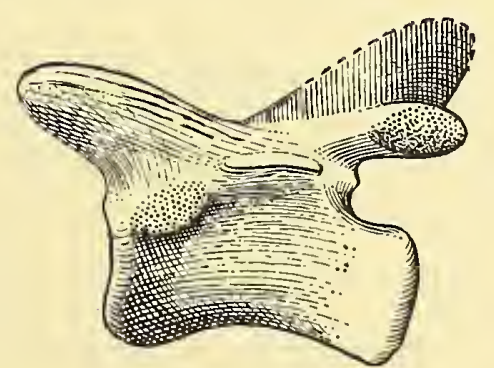

TEXT-FIG. 45.-Oligokyphus. Thoracic vertebrae of the trunk. $\times 4$. A, Anterior thoracic vertebra, Region III, R.7324, in dorsal and lateral view. B, Sixteenth vertebra, Region IV, R.7330, dorsal and lateral view. C, Vertebra of Region V, R.7344, posterior and lateral view. D, Presacral vertebra, Region VI. R.7336, dorsal and lateral view. 
has no tuberculum and the parapophysis is at the upper anterior margin of the centrum. The notch between the postzygapophysis and the centrum is now subdivided by a tiny process which does not, however, develop in subsequent vertebrae into an anapophysis. The width of the centrum is two-thirds of the length.

Region $V$

Material: R.7334, R.7335, R.7338, R.7340, R.7342-44. Seventeenth to twenty-second vertebra (Text-fig. $45, \mathrm{C})$.

The spinous process is even shorter. The zygapophyses are strongly developed. The postzygapophyseal facet is directed outward and downward, thus inhibiting the rotation of adjoining vertebrae. The elongated and nearly horizontal diapophysis is situated near the notch between centrum and postzygapophysis. The parapophysis is still at the anterior margin of the centrum. However, whereas in the previous region the facet was vertical, allowing the rib to move in an antero-posterior direction, this articulation is now inclined backwards at an angle of $45^{\circ}$. The upper margin of the parapophysis has moved posteriorly, away from the anterior edge of the centrum. The vertebrae are platycoelous.

\section{Region VI}

Material : R.733I-33, R.7336, R.7337, R.7339, R.734I. Twenty-third to twenty-sixth vertebra, presacral region (Text-fig. 45, D).

These are the largest elements in the vertebral column. The spinous process no longer overhangs the postzygapophysis. The zygapophyses are again strongly developed. The postzygapophyseal facet faces in a general direction outward ; but the facet is convex, the supero-external part being almost vertical and the infero-medial part horizontal. The diapophysis is longer and narrower and the whole parapophysis has now moved away from the anterior margin of the centrum. There is a gap between the parapophysis and the diapophysis, though it is much smaller than in vertebrae of the fifth region. The width of the centrum is two-thirds the length.

\section{Sacral Vertebra.}

Material: R.7345-48, R.7354. Twenty-seventh to thirtieth vertebra (Text-fig. 46).

Specimens R.7346 and R.7347 are first sacral vertebrae. R.7345 consists of two ankylosed vertebrae, the posterior one not being the last sacral vertebra, and the anterior one not being the first. Thus there appear to have been at least four sacral vertebrae in Oligokyphus. The facet of the ilium is preserved only in R.7347 : It is not vertical, and rises against the sagittal plane at an angle of about $40^{\circ}$. The neural canal of the sacral vertebrae is more depressed than in the presacrals and the succeeding caudals. The massive sacral ribs have a low insertion on the sides of the centra. The first sacral still shows traces of anapophyses, the third sacral does not.

Summarizing the account of the vertebral column posterior to the axis, the following points may be noted :

All vertebrae bear ribs as in Cynodonts. In Monotremes the two posterior presacrals 
are devoid of ribs. The ribs articulate as in modern mammals and not as in Monotremes. No ankylosed posterior presacral ribs are found as in all Cynodonts. The differentiation of the vertebral column approaches a mammalian condition. It is more pronounced than in the Monotremes and all older Therapsids. The vertebrae increase in length from the front backwards. There is a thoracic region where vertebrae can rotate around their longitudinal axis, a feature attained for the first time among Therapsids. All the spinous processes between the first thoracic and the last presacral vertebra point backward as in Tachyglossus and, probably, in Cynognathus. The spinous processes of the cervicals are very short and erect. There is no anticlinal vertebra. The exit of spinal nerves is through an intervertebral groove and not through a foramen as in Monotremes.
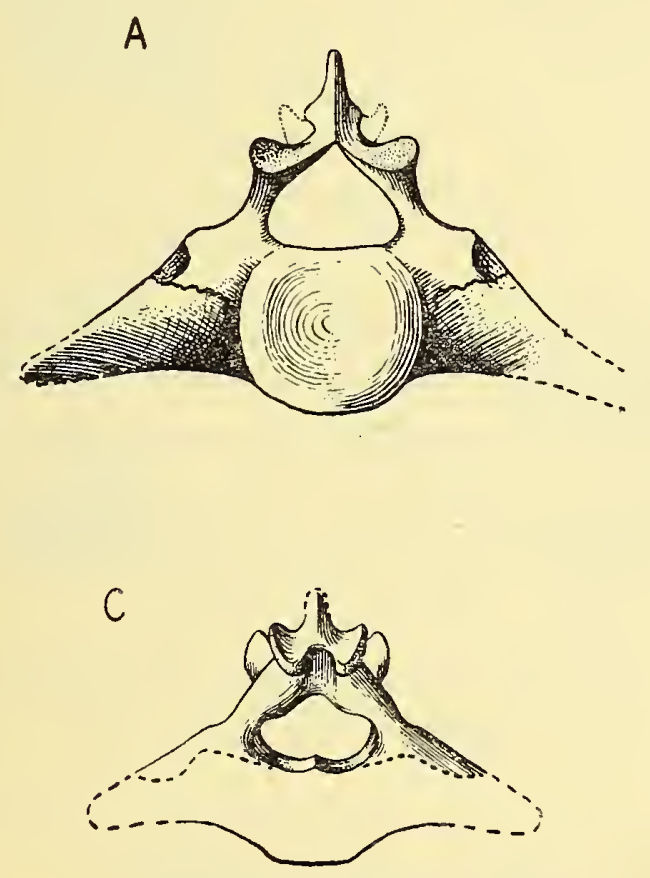
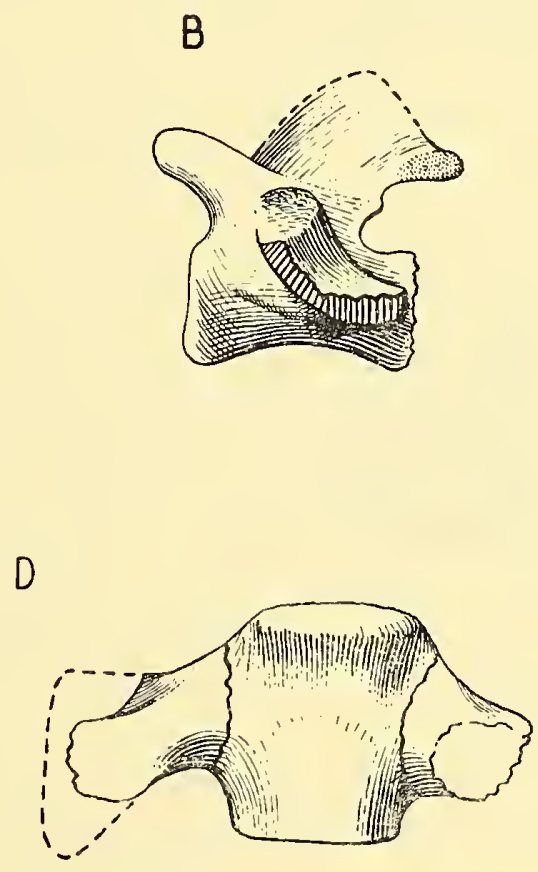

TEXT-Fig. 46.-Oligokyphus. Sacral vertebrae. $\times 4$. A, B, D, First sacral vertebra, R.7347. A, Anterior view. B, Lateral view. D, Ventral view. C, Sacral vertebra, R. 7345, posterior view.

The length of the vertebral column from the occipital condyle to the posterior end of the sacrum is $26 \mathrm{~cm}$. in Oligokyphus major, assuming a column of thirty elements with intervertebral discs I mm. thick.

There is no character in the spinal column of Oligokyphus which would debar it from being an ideal prototype for the Theria. The vertebrae of Oligokyphus are devoid of hypapophyses, metapophyses, anapophyses and secondary postzygapophyses. With movable ribs extending to the sacrum it is understandable that the diapophyses of the fifth and sixth region are very short. The functional implication of the absence of these vertebral processes is that the trunk musculature of Oligokyphus is weak and undifferentiated and that the animal's locomotion was slow compared with that of most placentals and marsupials. Rotatability of the anterior thoracic vertebrae has never before been observed in a Mesozoic Therapsid and as a true mammalian character is in itself interesting and significant. No 
functional explanation of this fact seems to be in the literature, not even in the papers of H. Virchow.

The functional implications of rotatability of the anterior thoracic vertebrae would appear, in order of their importance, to be as follows : (I) ability for an animal to sleep on its side or alternatively to move in and out of the lateral position. No other reptile can lie on its side ; (2) such a vertebral column is desirable and perhaps even necessary for an animal which has to clean its fur.

\section{The Tail}

Accounts of the osteology and myology of the tail are rare, unless this organ has a special function which is easily observed in the living animal. Many well-preserved tail vertebrae of Oligokyphus can be studied, but the tail does not show any striking adaptations. A long tail is usually, but not always, present in small and swift mammals; if however it is absent it does not necessarily mean that the mammal is slow. The tail of Oligokyphus is almost as long as its trunk; it was certainly not used for swimming as in Potamogale; there are no high spinous processes, no elaborate chevron bones. This tail has no long transverse processes as have the tail vertebrae of Ornithorhynchus and Castor. It has no similarity with a long reptilian tail, in which the vertebrae have long spinous and transverse processes and which is undifferentiated from beginning to end. This means that a typically reptilian muscle, the M. caudo-femoralis longus, is absent in Oligokyphus, a fact corroborated by the absence of the internal trochanter on the femur. The tail vertebrae of Oligokyphus are highly differentiated and the distal vertebrae are devoid of transverse processes, spinous processes and zygapophyses. In fact this is an essentially mammalian tail. It is here suggested that such a tail may be used, if densely covered with long hair, as an insulating organ during sleep, and that its movable proximal part may permit a mammalian form of coitus, which in the living reptiles is impossible because of the thick and relatively stiff root of the tail.

Each tail vertebra described is not designated to its exact position in the column, but the sequence of groups of vertebrae is correct as regards relative position.

\section{Group A}

Material : R.7349-R.7352 (Text-fig. 47, A-C).

This group contains vertebrae designated as proximal caudals. A number of criteria, taken together, make it clear that the designation is correct: (I) the neural canal in these vertebrae is narrower than in the sacral vertebrae; (2) the proximal caudal vertebrae have the postzygapophyseal facet anteriorly detached (Text-fig. 47, A). The presacrals and sacrals have this facet undetached. (3) The longest proximal caudal vertebrae are shorter than the longest presacral vertebrae. In mammals it is the posterior presacrals which are the longest vertebrae. (4) The proximal caudal vertebrae grade into similar ones which are obviously distal. These latter have a deep and medial groove for the coccygeal artery and vein and the ventral margin of the centra is bevelled to accommodate large chevrons.

Pre- and postzygapophyses are strong; the postzygapophyseal facet is concave. The prezygapophyses overhang the centrum. The spine is erect and short. The pleurapophyseal suture is distinct. The pleurapophyseal contact measured vertically is less exten- 
sive than it is in the sacrals and it decreases in Groups B and C. The pleurapophysis is nearly as long as the centrum. Length and breadth of the centrum are equal. There are no indications of chevrons.

A

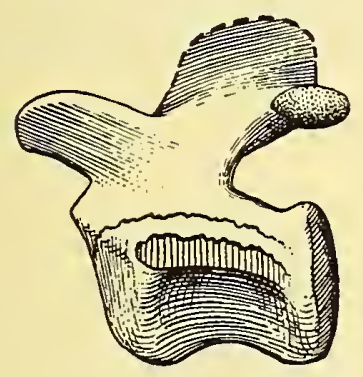

D

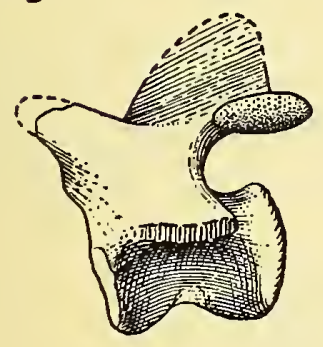

B

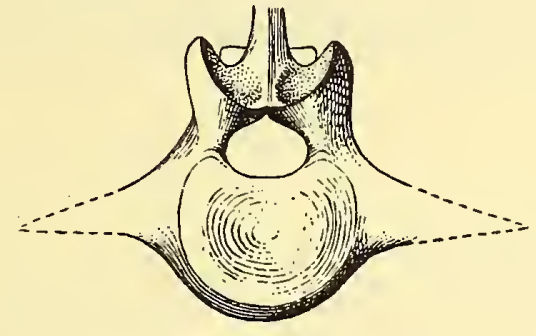

$C$
E

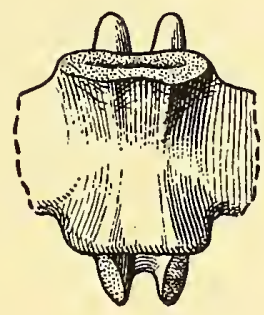

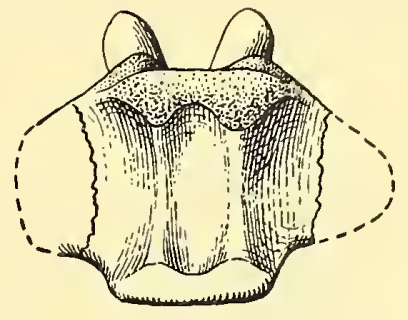

$\mathrm{F}$

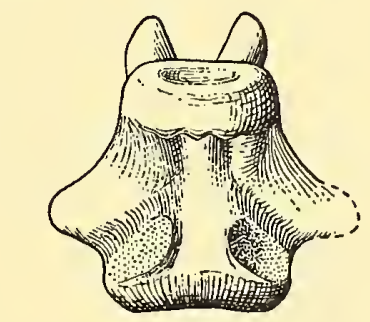

G

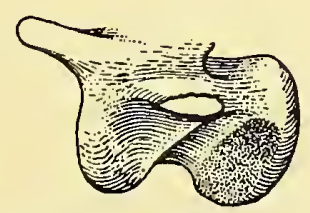

H

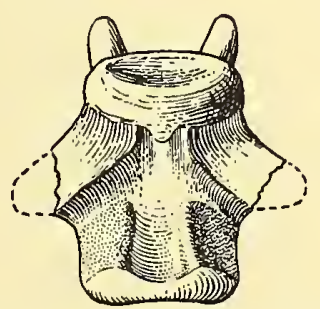

TExT-FIG. 47.-Oligokyphus. Proximal tail vertebrae. $\times 3$. Group A, R.7352: A, Lateral view.

B, Posterior view. C, Ventral view. Group B, R.7353 : D, Lateral view. E, Ventral view. Group B, R.7355 : F, Ventral view. Group C, R.7357 : G, Lateral view. H, Ventral view.

\section{Group B}

Material: R.7353, R.7355 (Text-fig. 47, D-F).

Vertebrae of this group are distinguished from Group A by three characters: (I) they are slightly more elongated ; (2) they have large chevrons-borne out by the bevelling of the anterior rim of the centrum ventrally; (3) they have a deep longitudinal groove ventrally for the coccygeal artery and vein. This groove has on each side a lateral branch following 
the curvature of the centrum supero-posteriorly and ending behind the pleurapophysis, a feature readily seen in mammals with strong and long tails. Consequently the centra of these vertebrae are deeply hour-glass shaped in lateral view. As the insertion area for the ventral tail muscles is thus restricted, the four surfaces between the vascular grooves are slightly roughened. The pleurapophysis is smaller in every direction than in Group A and points postero-laterally. In Groups $\mathrm{B}$ and $\mathrm{C}$ the planes through the anterior and posterior faces of the centra converge ventrally, indicating that the tail had a ventral concavity in these regions.

\section{Group C}

Material: R.7356, R.7357 (Text-fig. 47, G, H)

The postzygapophyses are broken off in both specimens and none is preserved in any more distal vertebra. Group E, however, does not develop any postzygapophyses at all. In these two vertebrae the transverse processes (the term is here used in a topographical sense) are, as in Group B, in front of the lateral vessels branching off the coccygeal artery and vein. No sutures are, however, visible, and it is possible that the processes are actually part of the centrum and not ribs. Indeed, in Group D, the transverse process in front of the vessel extends to the anterior face of the centrum and is part of the latter. It seems certain that at least in this position it is no longer a costal element. The prezygapophyseal facets are still concave though slightly less so than in the preceding vertebrae. The posterior third of the centrum is not covered by the neural arch.

\section{Group D \\ Material : R.7358-R.736o (Text-fig. 48, A-C)}

These three vertebrae lack a spine and the pre- and postzygapophyses are no longer in contact. The neural canal extends along the whole length of the centrum and the spinal nerve does not issue behind the neural arch and through the intervertebral groove, but through a lateral foramen $\mathrm{I} \cdot 5 \mathrm{~mm}$. anteriorly. Through the transverse process, which extends along the whole length of the centrum, run two vascular canals. The posterior canal is the continuation in latero-posterior direction of the lateral groove which branches off the medial groove for the coccygeal artery and vein. The lateral walls of the posterior part of the medial groove are especially high and the lateral surfaces for muscle insertions are distinct. The tranverse process issues almost from the ventral margin of the centrum, as do the sacral ribs. Measuring the breadth of the centrum is not satisfactory because the three specimens are not good, and the wide transverse processes, though they do not take part in the articulation of the centrum, are not clearly demarcated from them. The vertebrae of Group D are more elongated than those of Group C. This group, which may consist of one or two vertebrae, agrees to an astonishing extent with the fifth caudal of Didelphis.

\section{Group $E$}

Material: R.736r-66, R.7369 (Text-figs. 48, D-G, 49, A-D). Distal tail vertebrae.

The proximal elements of this group still have a spinal canal, but it is very narrow. The dorsal edge is always present. The shape of the centra, especially their ends, is not circular but rather triangular, the base being ventral. This indicates that the distal two-thirds of 
the tail was preferably moved in a dorso-ventral direction, the tail being flexed above the horizontal. Lateral movement was restricted.

Analysis of a tail can be made easier by plotting length against minimum breadth of its vertebrae. Oligokyphus lies between Lemurs, where successive vertebrae, beyond the fifth, get thinner but only little shorter, and Ateles where the corresponding vertebrae get shorter but only a little thinner. By making a graph of these data reliable conclusions can be drawn on the function of the tail, even if only a few complete vertebrae of the fossil mammal

A

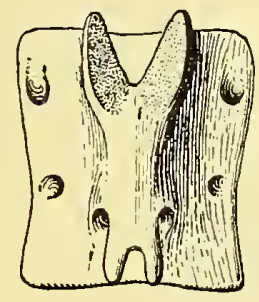

D

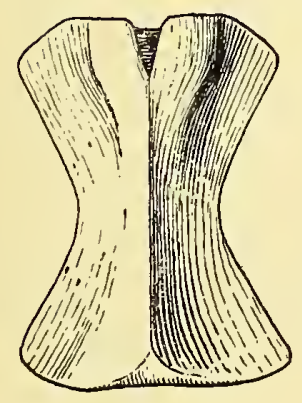

B

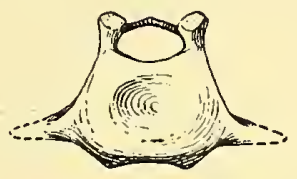

$E$

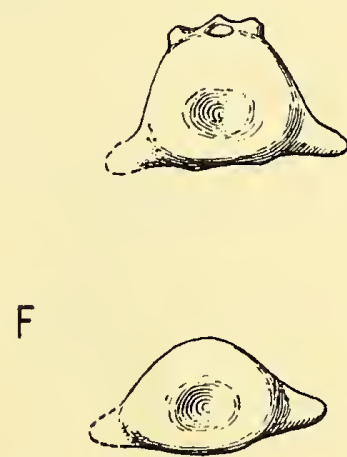

C

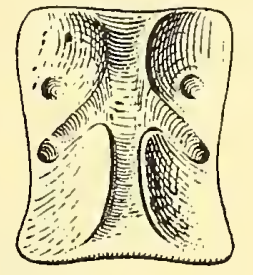

G

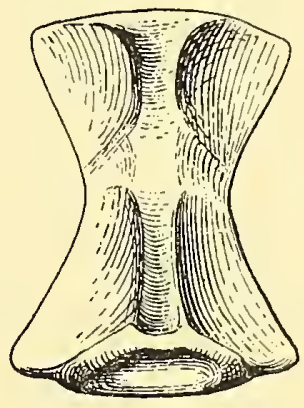

TExT-FIG. 48.-Oligokyphus. Tail vertebrae. $\times$ 3. Group D, mainly after R.7358: A, Dorsal view. B, Anterior view. c, Ventral view. Group E, R.7363: D, Dorsal view. E, Anterior view. F, Posterior view. G, Ventral view.

are available. If the last five tail vertebrae of Oligokyphus were as thick and short as those of Didelphis such vertebrae would have been found in the matrix, just as numerous phalanges have been found. Thus it is considered unlikely, though not impossible, that the tail of Oligokyphus was prehensile. Certainly it was not a thin and extremely elongated balancer as is the tail of Lemurs.

Length of tail vertebrae:

$\begin{array}{rrr}\text { Group A } & \text { No. R.7349 } & 6 \cdot 8 \mathrm{~mm} . \\ & , \quad 7350 & 5 \cdot 6 \quad, \\ & , 735 \mathrm{I} & 6 \cdot 8 \quad, \\ & , 7352 & 7 \cdot 4 \quad,\end{array}$


Length of tail vertebrae-contd.

\begin{tabular}{|c|c|c|c|}
\hline Group B & " & $\begin{array}{l}7353 \\
7355\end{array}$ & $\begin{array}{l}5 \cdot 9 \\
7 \cdot 0\end{array}$ \\
\hline Group C & ,, & 7356 & $8 \cdot 2$ \\
\hline & ", & 7357 & $7 \cdot 0$ \\
\hline Group D & ," & 7358 & $7 \cdot 7$ \\
\hline & ," & 7359 & $7 \cdot 8$ \\
\hline & ," & 7360 & $9 \cdot 5$ \\
\hline Group E & ", & 7361 & $10 \cdot 0$ \\
\hline & ," & 7362 & I0 8 \\
\hline & ," & 7363 & II $\cdot 7$ \\
\hline & " & 7364 & II $\cdot 8$ \\
\hline & $"$ & 7365 & 10.5 \\
\hline & " & 7366 & $8 \cdot 8$ \\
\hline & ," & 7369 & $10 \cdot 3$ \\
\hline
\end{tabular}

The discussion and identification of the tail vertebrae of Oligokyphus were done before measurement of the material and entirely on morphological evidence. The total number of tail vertebrae, though small and belonging to two forms of different size, nevertheless shows plainly that the proximal elements are shorter than the distal ones. The conclusions drawn from this evidence are: the proximal tail region has more joints per unit of length than the middle and distal region and hence could be flexed in a shorter arc. This agrees with a long mammalian tail which can be flexed flush with the back of the animal, only the five proximal tail vertebrae taking part in this flexion. A reptilian tail (e.g. that of Sphenodon, crocodiles and long-tailed lizards) consists of elements gradually decreasing in length from the first vertebra to the last. Though the tip of such a tail can reach the region in front of the head, the root of the tail is actually less flexible than the rest of it, due to its greater thickness and lesser number of joints per unit of length. Mammalian tail-wagging is out of the question.

\section{Cherron Bones}

Material: R.7370, R.737I and four unregistered specimens (Text-fig. 49, E-H).

Chevrons occur on all tail vertebrae with the exception of Group A. The proximal chevrons are well-developed and gradually become smaller at the same rate as do the vertebrae. They never have the length of the chevrons of Diapsids, indeed the tail of Oligokyphus was round in the flesh and more likely depressed than compressed.

\section{The Absence of Epiphyses on the Vertebrae}

Though sutures in the axis are still visible in all three specimens, the posterior face of their centra is well finished and does not show any trace of an epiphyseal suture.

None of the eight cervical vertebrae shows either fusion of the vertebral epiphyses or their loss due to maceration. 
Among twenty-two vertebrae belonging to regions I to IV, not one specimen shows loss of epiphyses due to maceration of the intervening cartilage, nor is it possible to observe a suture between the centrum and its anterior or posterior face.

The only first sacral vertebra in the collection has the sutures between its centrum and the sacral ribs still distinct, but its anterior face is well finished and no sign of a lost or recently fused epiphysis is visible.

All the numerous tail vertebrae have ends which do not indicate that either loss or fusion of epiphyses has taken place.

There are about a hundred additional and unregistered vertebrae which are either poorly preserved or have been damaged during preparation. Not one of these vertebrae shows ends which indicate that an epiphysis was lost or had recently fused. Not a single isolated epiphysis has been found in the matrix from Windsor Hill.

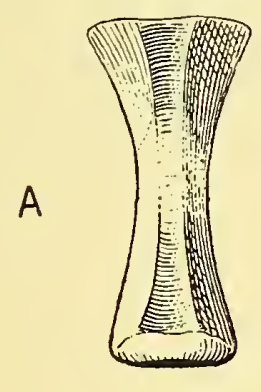

$E$

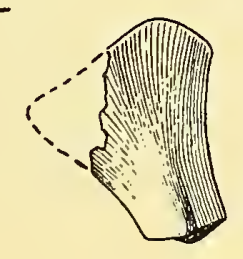

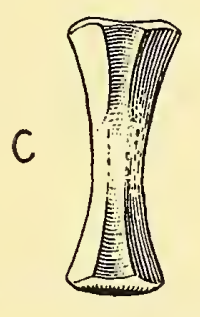

$\mathrm{F}$

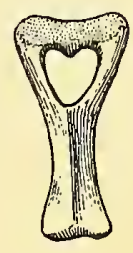

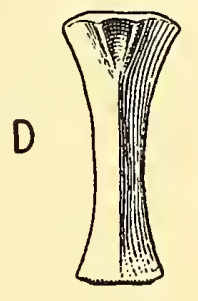

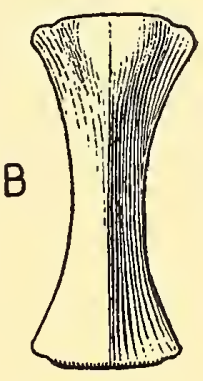

G

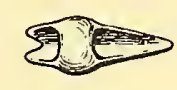

H

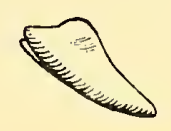

Text-FIG. 49.-Oligokyphus. Distal tail vertebrae and chevron bones. $\times 3 . \quad$ R.7369: A, Ventral view. B, Dorsal view. R.7366 : C, Ventral view. D, Dorsal view. R.7370, proximal chevron bone: E, Lateral view. F, Posterior view. R.737I, distal chevron bone: G, Dorsal view. H, Lateral view.

Since the rich series of vertebrae presumably contains some specimens from rather young individuals, the evidence is strongly in favour of the absence of vertebral epiphyses in Oligokyphus.

The sutures of the shoulder and pelvic girdle of Oligokyphus, like those of the cranium, are rarely fused. Moreover the dentition rarely shows signs of heavy wear, indicating that, in general, the animals were not very old when killed and that adult individuals tend to have their sutures still open.

If sutures between different skeletal elements and between diaphysis and epiphyses are obliterated at roughly the same time-and there is no means of confirming this-one would expect to find in the Oligokyphus material loose epiphyses or at least the sutures between 
diaphysis and epiphyses still visible, but this is not the case. Hence there were either no epiphyses in Oligokyphus or else the time during which epiphyses were still loose or the suture between them and the diaphysis still visible, was spent by the young in a burrow, hidden from the predators which lived on Oligokyphus.

\section{THE RIBS}

(Text-fig. 50)

Material: R.7374-R.7384. The rib material is poor; not one rib has the distal end preserved.

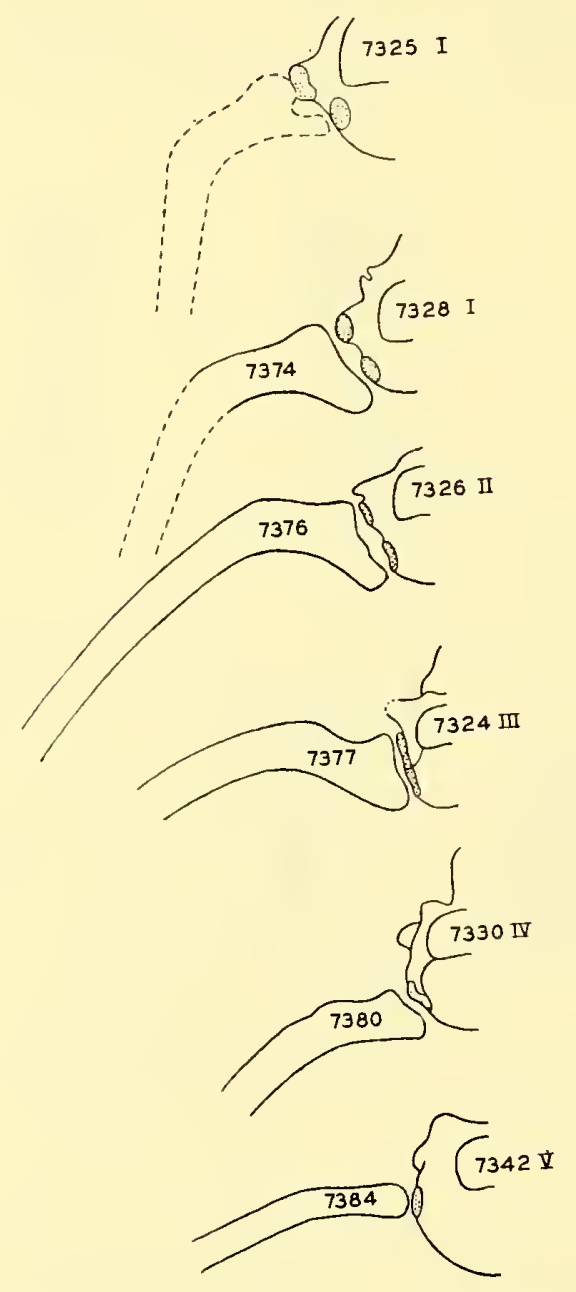

TEXr-FIG. 50.-Oligokyphus. Outlines of ribs in arbitrary association with vertebrae.

Many small mammals have ribs which are characterized by being curved in one plane only, the curve determining the surface of the thorax. They are flattened antero-posteriorly, the anterior side of the rib, if not convex, being at least less concave than the posterior side. The ribs are never round in transverse section. Distally from the tuberculum they are fitted with a distinct roughness for the insertion of the levator costae. Their distal half is straight. The ribs of Oligokyphus are of this kind and it has not been possible to detect any 
feature peculiar to them. It is probable that the ribs of Oligokyphus are not distinguishable from those of some other Therapsids of similar size or even from some other reptiles.

Proximal ends of double- and single-headed ribs occur.

The front view of the arbitrarily articulated ribs and vertebrae in Text-fig. 50 indicates that in transverse section the thorax was broader than deep, as it is in both genera of living Monotremes.

In the lateral views of the vertebrae one can see the articular surfaces for the ribs. In the vertebral regions I to IV the tubercular articulation is above and slightly behind the capitular articulation. Even among specimens belonging to the vertebral regions $\mathrm{V}$ and VI not one has been found in which the capitular articulation is horizontal. That means that during respiratory movement all ribs moved forward and backward to some degree and the ribs of the eight vertebrae behind the cervicals to such a degree as is required in a mammal. It may be assumed that in Oligokyphus costal and diaphragmatic breathing was as well established as, for instance, in the Monotremes. Comparing vertebrae and ribs of Oligokyphus with those of the Cynodonts, all of the latter show in the lumbar region specialisations or peculiarities not found in Oligokyphus nor in mammals. Gorgonopsids, on the other hand, have ribs and vertebrae from which one can easily derive those of Oligokyphus and the mammals.

\section{THE PRESTERNUM}

(Text-fig. 5I, A)

There is one complete specimen, R.7385. The bone is identical with that of Ornithorhynchus. The anterior margin is extremely thin and bilobed; the thin posterior margin of the interclavicle must have joined it without the provision of a distinct articular surface. The lateral margins of the bone are thick and sharp. At its antero-lateral corner there is a concave and round articular surface for the coracoid, the surface facing antero-laterally. The coracoidal articulation is smaller than in Ornithorhynchus. Immediately behind it is a small articular surface, facing laterally for the first rib. A similar surface is found at the

\section{A

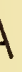

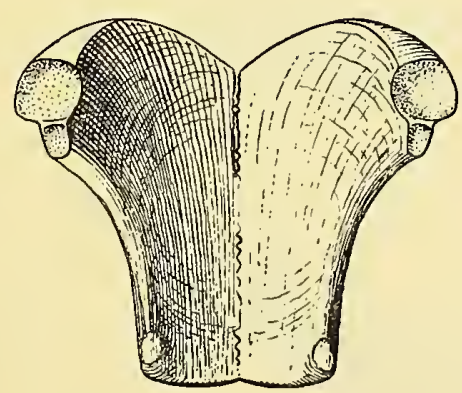

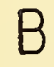

TEXT-FIG. 5I.-Oligokyphus. A, Presternum, R.7385, dorsal view, $\times 3 . \quad$ B, Sternum, R.7386, dorsal view. $\times 3$. 
postero-lateral corner of the bone. The ventral margin is about $\mathrm{I} \cdot 5 \mathrm{~mm}$. thick, and on it the sternum articulates. The distance between the centre of the coracoidal facet and the mid-line is $6.5 \mathrm{~mm}$.

\section{THE STERNUM}

(Text-fig. 5I, B)

There are only two specimens. R.7386 is a complete bone consisting of four co-ossified sternebrae; R.7387 is an isolated sternebra. Four pairs of ribs articulate with the sternum, so that there are six pairs of true ribs in Oligokyphus. The bone is dorsiventrally flattened and thus differs from the sternum of Ornithorhynchus, which is as thick as wide.

\section{The Interclavicle}

No interclavicle has been found in the matrix from Windsor Hill. It may therefore be presumed that it was thin, flat and rather small, as are the splenial and the angular, two important bones of the lower jaw, which likewise are still uncollected.

\section{THE CLAVICLE}

(Text-fig. 52)

Material: There are three specimens : R.7388 is an almost complete right bone measuring $24 \mathrm{~mm}$. ; R.7389 is the proximal half of a right clavicle; R.7390 is the proximal half of a left.

The clavicle is twisted, curved and flat ; one end is thin and slightly widened, the other end is rather massive. The thin end is the medial, in contact with the interclavicle, and the massive end is the lateral and in contact with the acromion. It is easy to determine the dorsal and the ventral sides of the bone, for one side is smooth along the whole length and is generally convex, and the other side is concave. The concave side has, at its medial end, striations indicating contact with the interclavicle and, at the lateral end, an articulation for the acromion. The former side would be ventral and the latter dorsal ; thus R.7388 must be a right clavicle.

A
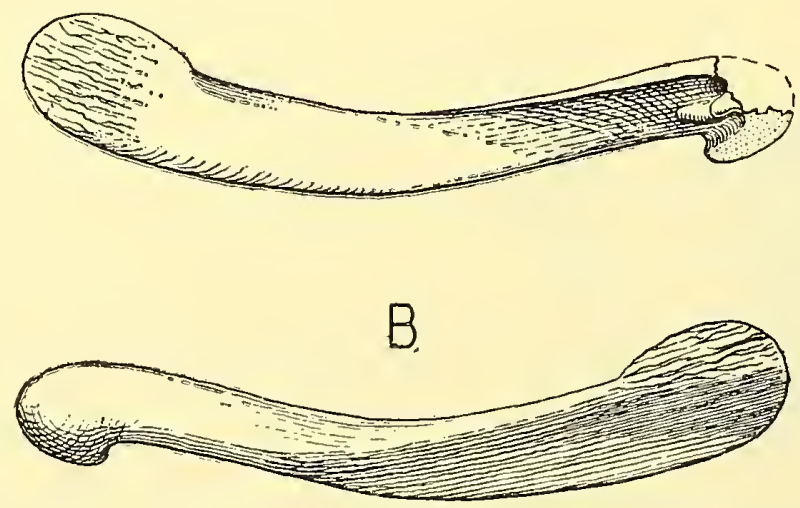

TeXT-Fig. 52.-Oligokyphus. Right clavicle, R.7388. × 3. A, Dorsal view. B, Ventral view. 
The contact between the clavicle and the interclavicle must have been exactly as in Cynodonts, and not as in the Monotremes, where the interclavicle is anteriorly expanded, forming a large T-shaped bone with the clavicles in contact with it along their posterior margin. The contact surface for the interclavicle of Oligokyphus is about $4 \mathrm{~mm}$. long. Assuming that both clavicles approximated in the sagittal plane, the anterior end of the interclavicle would have a width of about ro $\mathrm{mm}$. The lateral end is not satisfactorily preserved and a few millimetres of it are missing. The distal $4 \mathrm{~mm}$. of the dorsal surface bear several tubercles and on the posterior margin an articular surface for the acromion. The length of the bone preserved is $24 \mathrm{~mm}$. and, together with the width of the presternum, permits determination of the spread of the scapulo-coracoid.

\section{THE SGAPULO-CORACOID}

(Text-fig. 53)

Material: R.7385-R.7399 and seventeen unregistered fragments. Only two specimens, R.7395 and R.7396, have the scapula and coracoid co-ossified. There are three specimens of the precoracoid, but it is satisfactorily preserved only in R.7393; in all three the bone is suturally connected to the coracoid. The scapulo-coracoid material is poor. The upper half of the scapula and the lower end of the coracoid are unrepresented; the specimens on which the reconstruction of the bone-complex is based are from the right as well as from the left side and overlap but little. In consequence the reconstruction is made with reserve. In any case, the small size of the fragments has made their manipulation difficult.

The complete scapulo-coracoid of Diademodon in the collection of Professor D. M. S. Watson and the same bone-complex of both Monotremes have been used for comparison.

From Middle Triassic to late Mesozoic times the major stages in the evolution of the Therapsid scapula can be enumerated as follows: (A) The straight blade of the scapula becomes externally concave and the spinal part develops. (B) The acromion as a distinct process develops by drawing forward and undercutting the articular area for the clavicle. (c) The prespinous part develops.

The Cynodont scapula would be at stage A; that of Oligokyphus and the Monotremes at $\mathrm{B}$; and the Therian scapula would be at stage $\mathrm{C}$.

To produce an illustration of this twisted bone-complex has been difficult. The long axis of the complex has been erected vertically and an approximately lateral view has been taken with the glenoid cavity facing the observer. The frontal and medial views have been obtained by turning the bone through angles of $90^{\circ}$ and $180^{\circ}$ respectively. The glenoid cavity, however, is not wholly lateral but faces slightly backward.

Orientation of the scapulo-coracoid to its natural position has been accomplished by its articulations with the presternum and the clavicle. Its long axis rises forward at about $45^{\circ}$ and diverges from the sagittal plane at $40^{\circ}$.

The following are the salient features of this bone-complex: The glenoid fossa is formed by the scapula and the coracoid, the latter having the major share. The precoracoid, with its foramen near the suture with the coracoid, is a small triangular bone, smaller than in Diademodon, and, as in the latter, triangular in transverse section.

There are essential differences, as compared either with Diademodon or Ornithorhynchus, in the central part where the three bones meet. In Ornithorhynchus the precoracoid does 


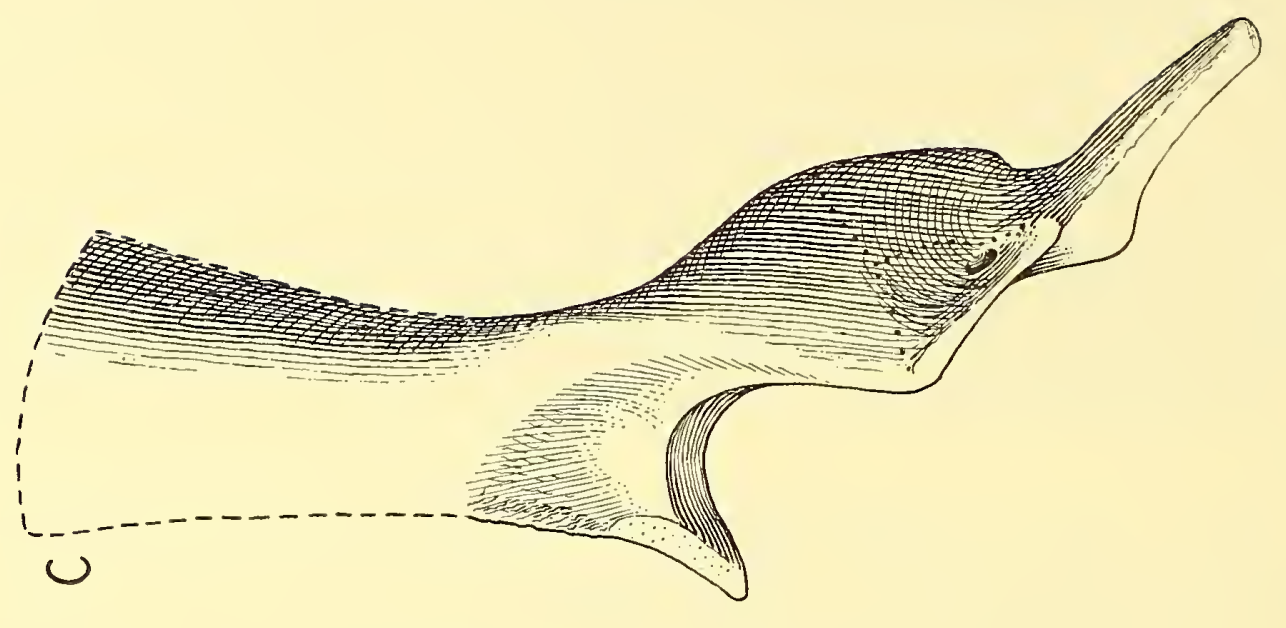

Е

ले

$\approx$

ब

i

ธ

큥

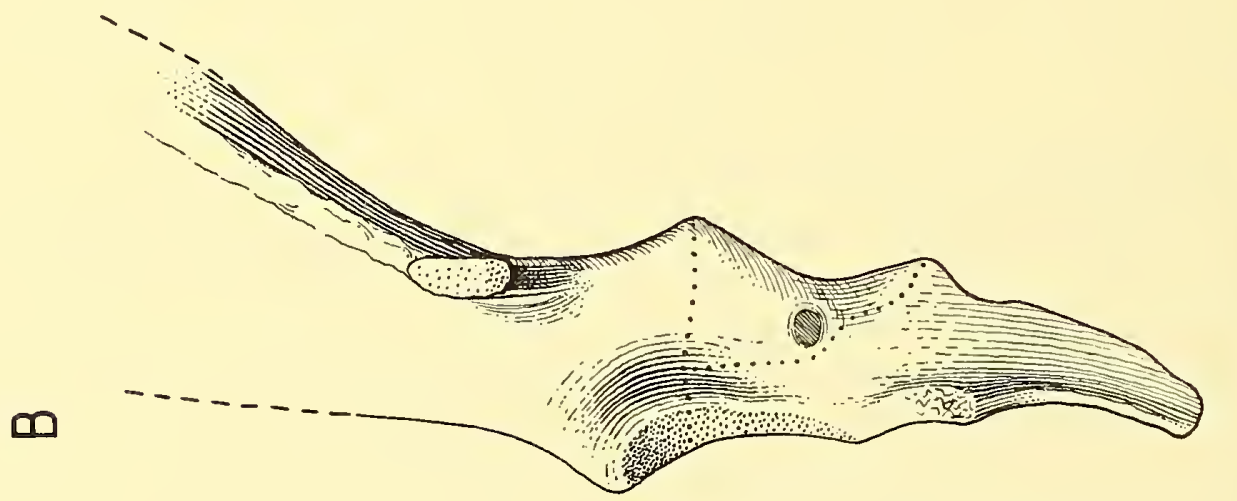

承究

in

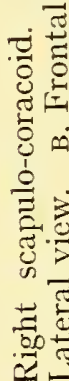

s 4

空

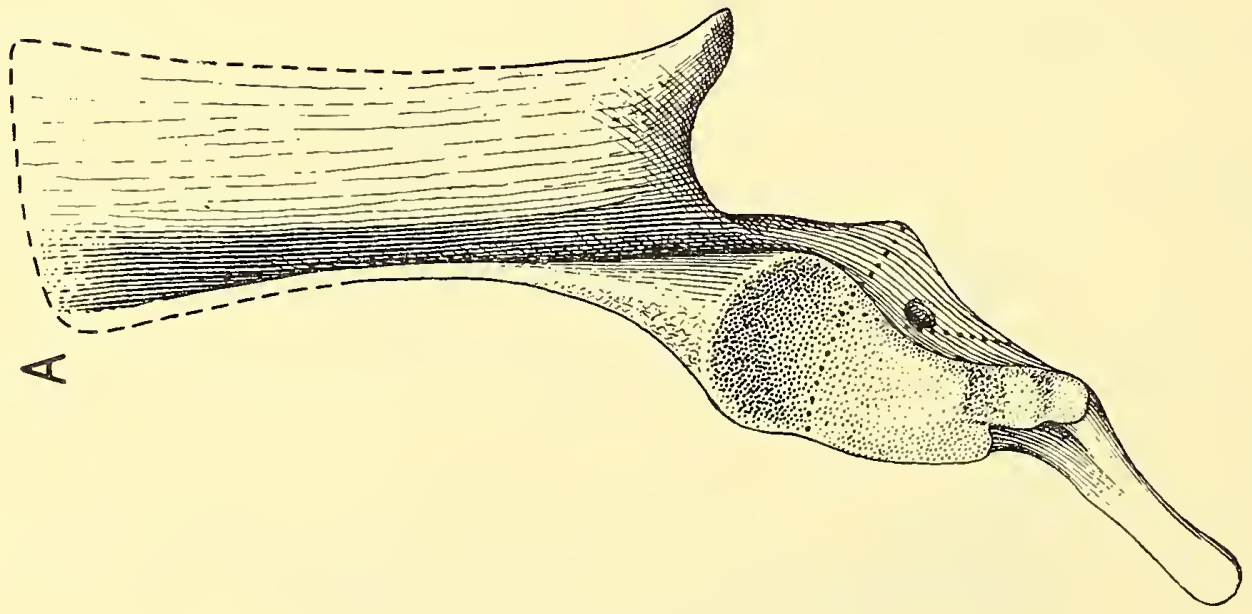


not articulate with the scapula ; in Diademodon the precoracoidal-scapular contact is wide, the bone-complex having its greatest width at this suture. In Oligokyphus too the precoracoidal-scapular contact is long, but above it the latero-median width of the scapula decreases up to the point where the spinal part of the blade rises almost horizontally from the main body of the scapula. The scapula of Oligokyphus is laterally concave as in Diademodon. The large acromion of Oligokyphus is entirely free as in the Monotremes. The prespinal part of the scapula is absent in Diademodon, Oligokyphus and Monotremes. In Ornithorhynchus, however, a longitudinal ridge on the medial side of the scapula may be called an incipient prespinal part. This ridge divides the insertion area for the M. subscapularis from that of the M. serratus magnus and M. acromio trachelius. In Oligokyphus a similar ridge is present owing to the change of curvature of the scapula. Here the insertion area for the subscapularis is convex, that for the M. serratus magnus is concave.

\section{Muscle Insertions on the Scapulo-coracoid}

No dissection or review of the shoulder musculature of Monotremes has been done, and identification of insertion areas is based on the papers by McKay (I895), Wilson \& McKay (I894), Gregory \& Camp (I9I8) and Watson (I9I7). Only those muscles are mentioned whose insertion areas are distinct in the material.

On the lateral side, the deep and long concavity formed by the spine and the postspinal part of the scapula houses the strong M. infraspinatus. The M. triceps has a good insertion area above the glenoid fossa.

On the medial side the insertion of the M. subscapularis and M. serratus magnus have already been mentioned. The free acromion has on its lower and medial face a very distinct crescent-shaped scar, well separated by ridges above and posteriorly. This scar is the insertion area for the supraspinatus. It is noteworthy that the undercutting of the acromion and the presence of the supraspinatus-two correlated features-actually occur together in Oligokyphus.

The intermediate position of the scapulo-coracoid of Oligokyphus between those of Diademodon and the Monotremes is striking. There is no character in Oligokyphus which goes beyond a condition from which the Monotreme scapulo-coracoid could be derived; nor are there any characters in Oligokyphus which show specialization.

\section{FORE LIMB}

\section{THE HUMERUS}

(Text-fig. 54)

Material: There are sixteen registered specimens, R.7400-I5, and twenty-five unregistered of this bone. Altogether forty-eight specimens have been prepared. R.7400-03 and R.74I5 are entire; R.740I lacks the head and the ectepicondyle, but has an excellently preserved distal end ; and R.7403 was the only uncrushed and intact proximal end. Twentyone measurements of the minimal thickness of the shaft have been taken ; they do not show bimodality.

Length of the humerus :
R. $7400,36 \mathrm{~mm}$.
R.7402, $40 \mathrm{~mm}$.
R.74I5, $37.5 \mathrm{~mm}$. 
This bone resembles the "Stonesfield humerus" (Simpson, I938: I55) in all characters displayed by that specimen.

Neither the lesser nor the greater tuberosity is detached from the head, the cartilagecovered surface of the head being confluent with both. As in Cynodonts the lesser tuberosity is by far the larger ; it is rounded on top and its distal prolongation is distinct down to the middle of the bone. The lesser tuberosity shows two well-marked muscle scars, one at its proximal end for the M. subcoracoideus and another, about Io $\mathrm{mm}$. distal, for M. subscapularis, M. teres major and the internal head of M. triceps. The bicipital groove is as wide as in Cynodonts, and reaches two-fifths of the way down the shaft, where its end is distinctly marked by a large scar for the insertion of M. coraco-brachialis brevis.

The delto-pectoral crest is very thin and very high, and is continued for almost the whole length of the bone as a smooth high ridge, traversing the bone and ending at the upper margin of the long foramen entepicondyloideum.

On the dorsal side of the bone, distally to the head, is a faint ridge running longitudinally and dividing the areas of origin for the external humeral head of M. triceps and M. brachialis internus. The supinator crest is sharp and wide and there is an extensive insertion area for M. anconeus.

The twisting of the shaft results in an acute angle being formed between the long axes of the distal and proximal ends. This angle, about $40^{\circ}$, is similar to that in Diademodon. The delto-pectoral crest and its distal prolongation make the shaft subtriangular in transverse section.

The radial and ulnar articulations are confluent, and nowhere is there a sharp edge dividing the two regions from the entepicondyle or the ectepicondyle. The ulna articulates only on the dorsal side. On this dorsal side the medial rotundity is larger than the lateral, a shallow groove separating the two.

Ventrally the radial and ulnar articulations are side by side, the radial articulation being hemispherical and much larger than the ulnar one. The ectepicondyle is narrower than the radial articulation and is thus well separated from the latter, which is not the case in Diademodon. There is no ectepicondylar foramen in Oligokyphus.

The Stonesfield humerus is considered here to belong to Stereognathus. Seeley, when describing the humeri of "Microgomphodon" and Gomphognathus did not refer to the Stonesfield specimen, which he had described earlier, and he made no mention of the essential similarity of the three humeri.

Broili \& Schröder (I936a) describe the distal half of the right humerus of Tritylodon, and the new evidence corroborates their identification. All the characters which the specimen shows agree with Oligokyphus.

Young mentioned the humerus of Bienotherium twice. Though quite rightly described as a left humerus in his first paper (I940), the specimen VII is identified as a right in his second paper (I947: 582).

The humerus of Oligokyphus differs in the following characters from Diademodon: head spherical, extending well on to the dorsal surface; delto-pectoral crest shorter; no ectepicondyle foramen; entepicondyle less extensive.

The humerus of Oligokyphus differs in the following characters from Therian humeri: epicondyles expanded; deltoid crest longer and deeper; bicipital groove much wider; lesser tuberosity more extensive than greater tuberosity; articular region on distal end 

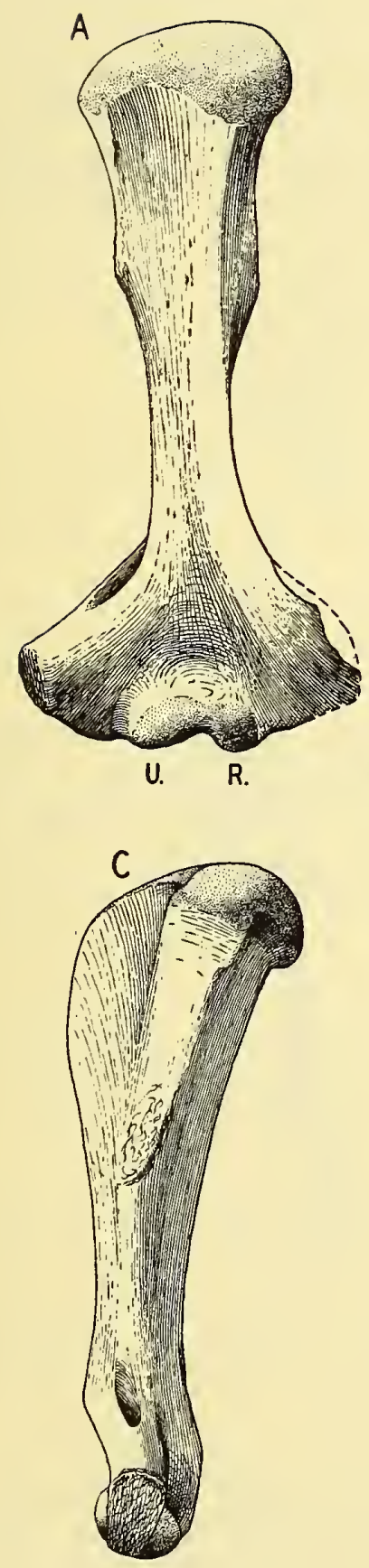
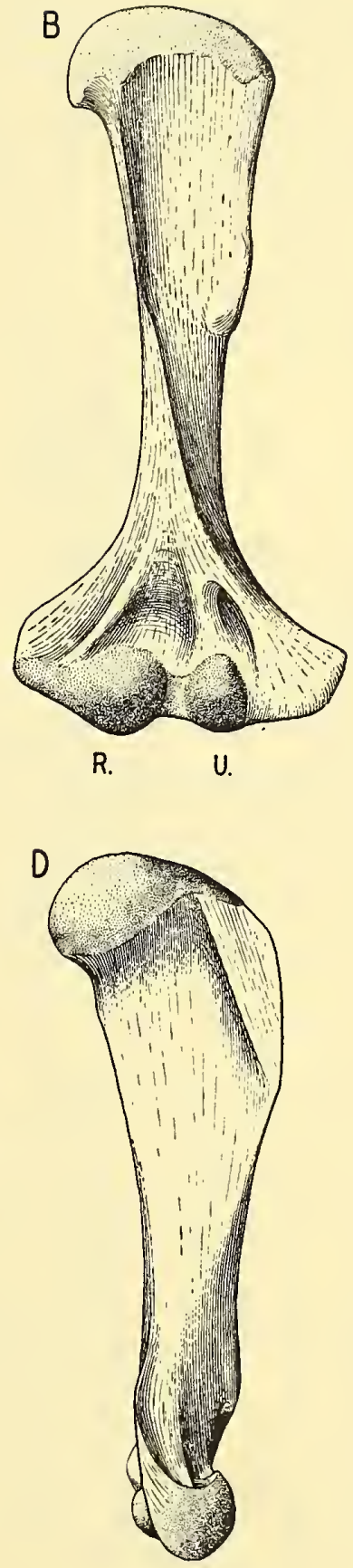

Text-FIG. 54.-Oligokyphus. Right humerus. $\times 2$. Based on R.7400, R.740r, R.7403 and R.7404. A, Dorsal view. B, Ventral view. C, Medial view. D, Lateral view. E, Distal end of right and proximal end of left humerus. Three sections through shaft of right humerus. G.T., greater tuberosity ; L.T., lesser tuberosity ; $R$., radial articulation $U$. , ulnar articulation. 
confluent with epicondyles; angle of $40^{\circ}$ between long axes of distal and proximal ends.

Simpson, discussing the Stonesfield humerus, assumes for it a posture similar to that of the Cynodont humerus and allows the humerus to articulate with a scapulo-coracoid in which the latter element is still large. Since the scapulo-coracoid of Oligokyphus is essentially of Cynodont pattern, as is also its humerus, it is evident that the posture of the humerus of Oligokyphus was the same as in Cynodonts, viz. that the long axis of the bone was horizontal with the distal end pointing latero-posteriorly. The long axis of the distal end was also horizontal.

In Oligokyphus the length of the humerus is more than twice the distal width, whereas in Bienotherium the length is less than twice the width of the distal end.

\section{THE ULNA}

(Text-fig. 55)

Material: R.74I6-743I and many unregistered fragments.

Length as preserved:

R.74I6, $40 \mathrm{~mm}$.

R.7426, $34 \mathrm{~mm}$.

A restored ulna of Oligokyphus major measures $50 \mathrm{~mm}$. The only ulna which was complete in the matrix, R.7426, was damaged at both ends during preparation. Fortunately, some of the available specimens are very well preserved so that restoration can be confidently made. If the humerus and the ulna are compared, the preponderance of mammalian features in the ulna is overwhelming. This structural difference between the two bones is extraordinary, and is another example of an unexpected combination of characters in one animal.

The humerus and the ulna of Oligokyphus are the more remarkable when one compares them with the same bones of Diademodon, where the ulna is strikingly reptilian. The left ulna, Eoraetia siegerti of Lower Rhaetic age, described by Dietrich in I937 is the only ulna readily comparable with Oligokyphus.

The olecranon is long and massive. The proximal surface is rounded, rough and without a groove for the M. triceps tendon. The medial and anterior faces of the olecranon are sharply divided by a vertical edge. The medial margin of the fossa sigmoidea is protracted above and below (processus coronoideus), the middle part being constricted and confluent with the shaft. The articular surface is concave supero-inferiorly; it is also concave lateromedially with the exception of a narrow strip on the lateral side. This convex area rises imperceptibly from the radial articular surface to the superior margin of the fossa.

The articular surface for the radius is triangular and directed forward and outward; it has the same surface characters as the other parts of the sigmoid fossa, which means that it was covered by cartilage. The articular facet for the radius is slightly concave lateromedially. The lower margin of this facet is sharp. Below the junction of the radial and the humeral articulations there is a small but distinct fossa for a ligament, and below and medially of the processus coronoideus there is a long and distinct tuberositas ulnae for M. biceps and M. brachialis.

The shaft is evenly sinuously curved and flattened latero-medially. The antero-posterior 
width is almost constant from below the coronoid process to the distal end. The posterior margin is rounded along the whole length and the anterior margin of the shaft is also rounded in the proximal half, but thin and even sharp in the distal half. The medial side of the shaft is slightly concave and otherwise featureless. The upper half of the lateral side is concave, the long depression being bordered anteriorly by the tuberositas ulnae. Before this concavity disappears distally, a smooth and rather high ridge rises at the anterior border running obliquely down the shaft and gaining in height. In the distal sixth of the shaft this ridge loses height as well as distinctness, but between it and the anterior margin there is now a well-defined longitudinal concavity which accommodates the radius.
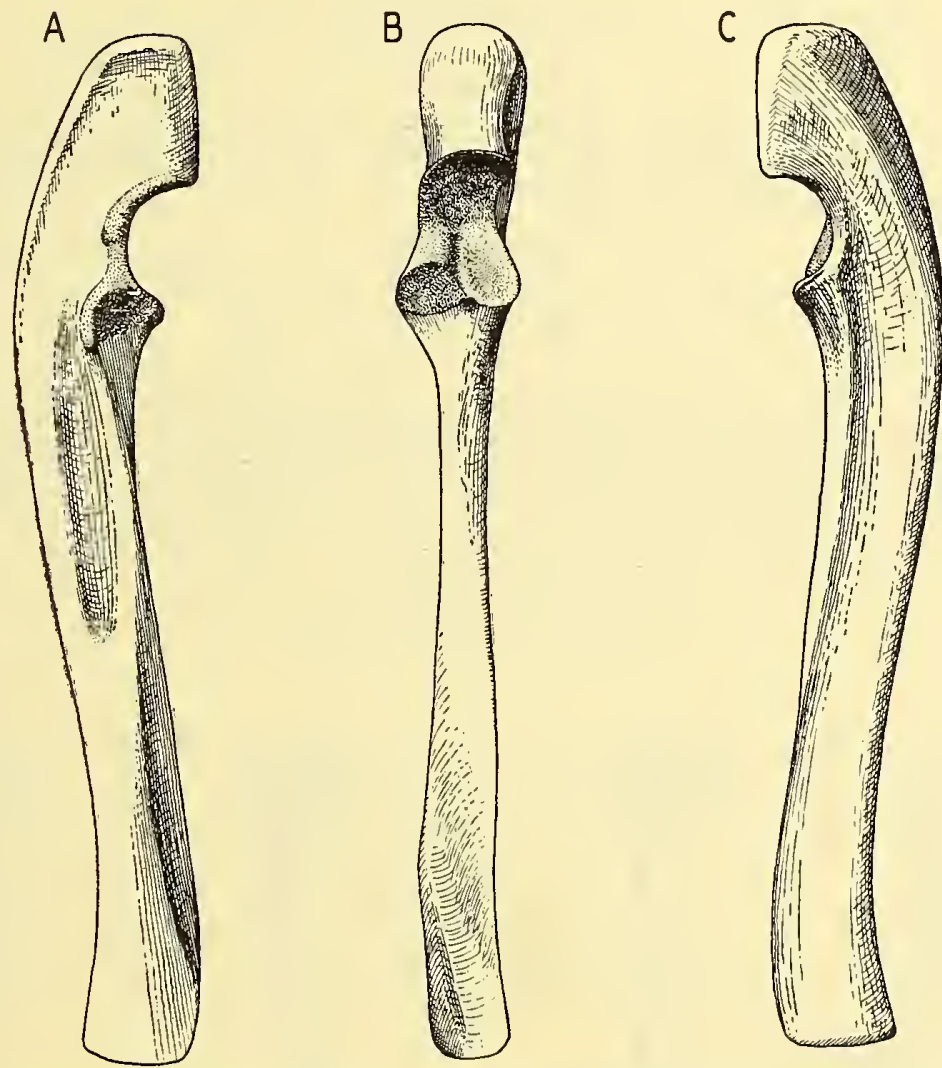

TEXT-FIG. 55.-Oligokyphus. Right ulna. $\times$ 2. Based on R.74I6, R.74I7, R.74I9, R.7420, R.7427. A, Lateral view. B, Anterior view. C, Medial view.

There is only one well-preserved distal end, R.7420. The outline of the articular surface is semicircular, the flat base of the semicircle being medial. The concavity which accommodates the radius does not reach the distal articular surface. There is a distinct ridge running latero-medially which divides a small anterior area from the main articular surface ; the latter is shallowly concave. There is no styloid process.

The head of the radius is lateral to the ulna and its distal end is directly in front of it. The radius can to some extent rotate round the ulna. Though the two bones do not meet distally on a rough plane surface, which would indicate a fixed position of the radius, and though there is a long groove in the ulna to house the radius, there are nevertheless no 
cartilage-covered articular surfaces on the distal part of the shaft of either bone such as occur in mammals.

Dietrich (I937: 3I4) supposed that the ulna of Eoraetia had a styloid process, but the essential similarity of the ulna in Oligokypluns suggests that the missing distal end of the former resembled that of the latter. Eoraetia differs only in a concave contact area for the radius along the anterior margin of the distal half of the shaft of the ulna. Dietrich deduced from this that the radius of Eoraetia was probably fixed in the pronate position. It seems true that the radius of Eoraetia had less freedom to rotate than that of Oligokyphus.

\section{THE RADIUS}

\section{(Text-fig. 56)}

Material: R.7432-7440, eight unregistered fragments, and a whole but crushed specimen in my own collection measuring $34.5 \mathrm{~mm}$. in length.

Length of radius :

R. $7435,33^{\circ} \mathrm{o} \mathrm{mm}$.

R.7432, $30.4 \mathrm{~mm}$.

The radius is S-shaped in posterior and straight in lateral view. The rim of the proximal end is not horizontal, but makes an angle of $20^{\circ}$ with the long axis of the shaft facing anterolaterally. The proximal articular surface is concave. The almost circular articular surface is elongated in antero-lateral medio-posterior direction. Below the rim, and on the medial side, there is a transverse articular surface for the radial facet of the ulna.

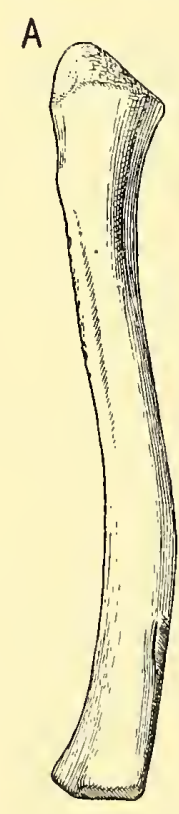

Lat.

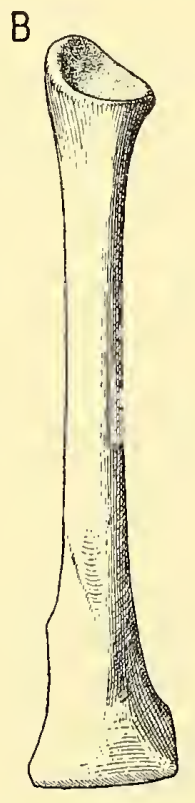

Ant

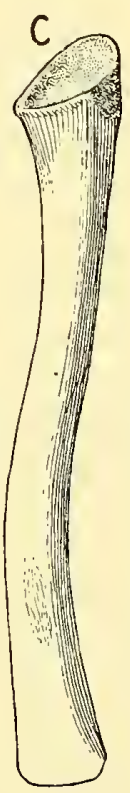

Med

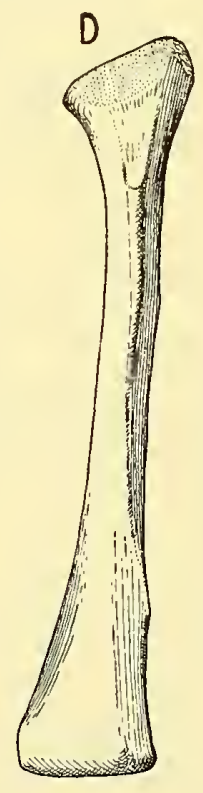

Post

TEXT-Fig. 56.-Oligokyphus. Right radius. $\times 2$. Based on R.7432 and R.7435. A, Lateral view. B, Anterior view. C, Medial view. D, Posterior view. 
Medial side. Beginning a few millimetres below the neck and terminating at the middle of the shaft, there is a narrow and rough ridge for the interosseous ligament. Anteriorly to the proximal end of this ridge is the tubercle for the insertion of M. biceps and M. brachialis, The distal third of the shaft widens antero-posteriorly.

Lateral side. The distal third of this side of the radius bears a shallow and smooth groove; beginning at the posterior margin it slants distally towards the anterior margin. It fades out before reaching the distal end. This oblique groove is a characteristic feature of mammals with a pentadactyle manus. In Homo this groove houses the bellies of M. extensor pollicis brevis and M. adductor pollicis longus. The groove is absent in mammals which lack the pollex.

Posterior edge. There is a sharp edge, beginning almost at the distal end and ending at the beginning of the groove just described.

Anterior side. There are a number of deep muscle insertions in the distal half. The distal articular end is subdivided latero-medially by a faint straight ridge, producing two shallow concavities of almost equal size. The anterior half is roundly rectangular, the posterior half is pointed posteriorly, the point being in contact with the ulna.

In conclusion, the antibrachium of Oligokyphus was well suited for supination and pronation, and the manus had a pollex which was presumably opposable. The intermedium was large, a small part of it articulating with the ulna. In Oligokyphus the distal end of the ulna bears a concavity for the accommodation of the radius. In the mammals it is always the radius which has a concavity for the convex articular surface of the ulna.

\section{THE PELVIC GIRDLE}

(Text-fig. 57)

Material: R.744I-746I and forty-one fragments which are unregistered.

Only one specimen, R.744I, consisting of the right ilium and pubis, has the two bones co-ossified. Dr. Broom examined this specimen in I949 and pointed out that it belonged to the right side and contains ilium and pubis.

The ilium is completely represented in the material; not so the ischium and pubis. No specimen contains even part of the symphysis and only one pubis R.7459, has a small

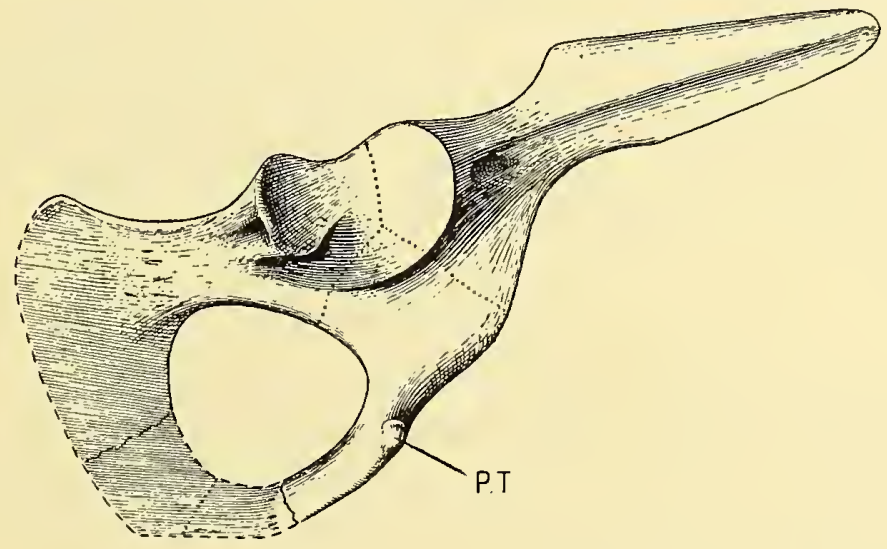

Text-Fig. 57--Oligokyphus. Right pelvis. $\times 2$. Based on R.744I, R.7448, R.7450, R.7455. P.T., pubic tubercle. 
area of the anterior part of the ventral margin preserved, which, in the Monotremes, articulates with the marsupial bone. No marsupial bone has been found in the matrix from Windsor Hill.

The main features distinguishing the pelvic girdle of Oligokyphus from that of Cynodonts is the absence of an iliac blade above and behind the acetabulum and the presence of a cotyloid notch in the ischial part of the acetabulum.

\section{THE ILIUM}

The anterior part of the ilium is a four-sided tapering bar. The lateral side is divided by the crista lateralis into an upper gluteal, and a lower iliac, fossa, and the medial side is also divided longitudinally. The articular surface for the sacral vertebrae reaches farther backward above than below. Both upper and lower margins of this surface are sharp; its oblique posterior margin is elevated. Posteriorly a very small part of this surface was either co-ossified with a sacral rib or a cartilage covered joint was present. There is evidence in seven specimens that both conditions existed. Anteriorly the sacral articular surface is deeply furrowed, indicating that ligaments intervened between this part of the ilium and the anterior sacral ribs. A macerated ilium of Didelphis shows identical conditions. The upper margin of the anterior part of the ilium was slightly inclined. Probably M. longissimus dorsi was not directly inserted to the inner side of the ilium. Below the posterior end of the crista lateralis there is a pit for the insertion of $\mathrm{M}$. rectus femoris. The three elements of the innominate bone have an equal share in the acetabulum; no trace of a cotyloid bone has been observed. The narrow cotyloid notch does not widen into an acetabular fossa ; apart from the notch, the whole surface of the acetabulum is articular.

\section{THE ISCHIUM}

This bone is very similar in Diademodon, Oligokyphus and the Monotremes. In the last the ischial tuberosity is drawn out, making the posterior and superior borders deeply concave. In the two Mesozoic forms the ischial tuberosity is less pronounced and in consequence the posterior border is convex. Apart from the presence of the cotyloid notch in Oligokyphus, there is only one other feature worth mentioning; in the Monotremes the ischium is smooth and flat behind the acetabulum. In Diademodon a strong ridge passes from the rim of the acetabulum to the ischial tuberosity and thus separates a smaller superior from a larger inferior part, the two surfaces meeting at an angle of $90^{\circ}$. In Oligokyphus. the ridge has migrated upward and the superior surface has shrunk.

The obturator foramen is as large as in Ornithorhynchus.

\section{THE PUBIS}

The antero-inferior part of the pubis is everted in the same manner as in Diademodon. This eversion is an ancient character found in Pelycosaurs and still present in Monotremes. In the latter it is made less obvious by the presence of the ilio-pectineal spine, which is lacking in the two fossil forms. The point of the everted anterior part of the pubis is the pubic tubercle ; it is abraded in all specimens, but in R.7459 there is preserved at least I $\mathrm{mm}$. of the inferior border behind the tubercle. This border is rectangular, but has rounded 
edges. Its inferior surface (there is only an area $I \times 0.5 \mathrm{~mm}$. observable) is devoid of compact bone and has the characters that indicate a cover of cartilage. The marsupial bone of Monotremes articulates on the inferior border of the pubis between the pubic tubercle and the symphysis. The meagre evidence is ambiguous as regards the presence or absence of a marsupial bone in Oligokyphus. Among the Theria the pubis suffers reduction and with few exceptions the anterior part becomes vestigial. In Oligokyphus this process has not yet begun and its pubis is hardly distinguishable from Diademodon.

The pelvic girdle of Oligokyphus goes beyond the Prototherian towards an approach to the Therian condition. In the Monotremes a large ileo-pectineal spine is present and the cotyloid notch is absent. In Oligokyphus the reverse is the case.

\section{HIND LIMB}

\section{THE FEMUR}

(Text-fig. $\left.5^{8}\right)$

Material: R.7462-7480 and a number of unregistered fragments.

Only in R.7467 is the entire length available; it is $42.6 \mathrm{~mm}$. The proximal end is beautifully represented by R.7465 which is extremely large; its estimated total length is $45.5 \mathrm{~mm}$. Not one complete distal end is preserved; the anterior condyle is abraded in all specimens.

The terms used for orientation of the bone are those of the primitive position of the tetrapod limb, ventral being the equivalent of posterior in Homo, dorsal that of anterior, anterior that of medial, and posterior that of lateral.

The flat ventral side is separated from the lateral and medial sides by a lower anterior edge beginning at the anterior (tibial) condyle and fading out $\mathrm{I} 3 \mathrm{~mm}$. above the distal end ; an upper anterior edge beginning at the tip of the lesser trochanter and fading out $18 \mathrm{~mm}$. below the proximal end; and a posterior edge beginning at the posterior (fibular) condyle and fading out at the greater trochanter. The shaft in general, however, is not flattened as, for instance, in the Monotremes and in Erinaceus, the dorso-ventral and the anteroposterior diameters being equal at the middle of the bone. There is no digital fossa, but the whole inter-trochanteric area is gently concave the ventral part of the caput overhanging it. The posterior part of the caput rises straight from the notch between it and the greater trochanter for there is no concavity below it. Anteriorly there is a faint constriction below the caput indicating an incipient neck. The lesser trochanter is anterior.

On the dorsal side, the region between the trochanters is as much convex as it is concave on the ventral side : a patellar facet is hardly noticeable.

The articular surface of the caput faces slightly upward and forward. A line connecting the trochanters is in the same plane as the antero-posterior axis of the distal end.

The condyles are not separated by a notch. The popliteal fossa is shallow. The patellar fossa is extensive and flat. There is no indication in the rather poor material that the femur was in articulation with the fibula.

The long axis of the femur is more or less horizontal, perhaps even slightly inclined towards the sagittal plane, the large lesser trochanter preventing the bone from being directed more than $20^{\circ}$ forward. 
Simpson's description of his "Stonesfield femur" (I928 : I49) could pass almost literally for Oligokyphus. This specimen is exposed only on the ventral side and it is obviously flattened by compression. The longitudinal groove which Simpson describes is due, in my opinion, to crushing, and is not found in Oligokyphus.

\section{A}
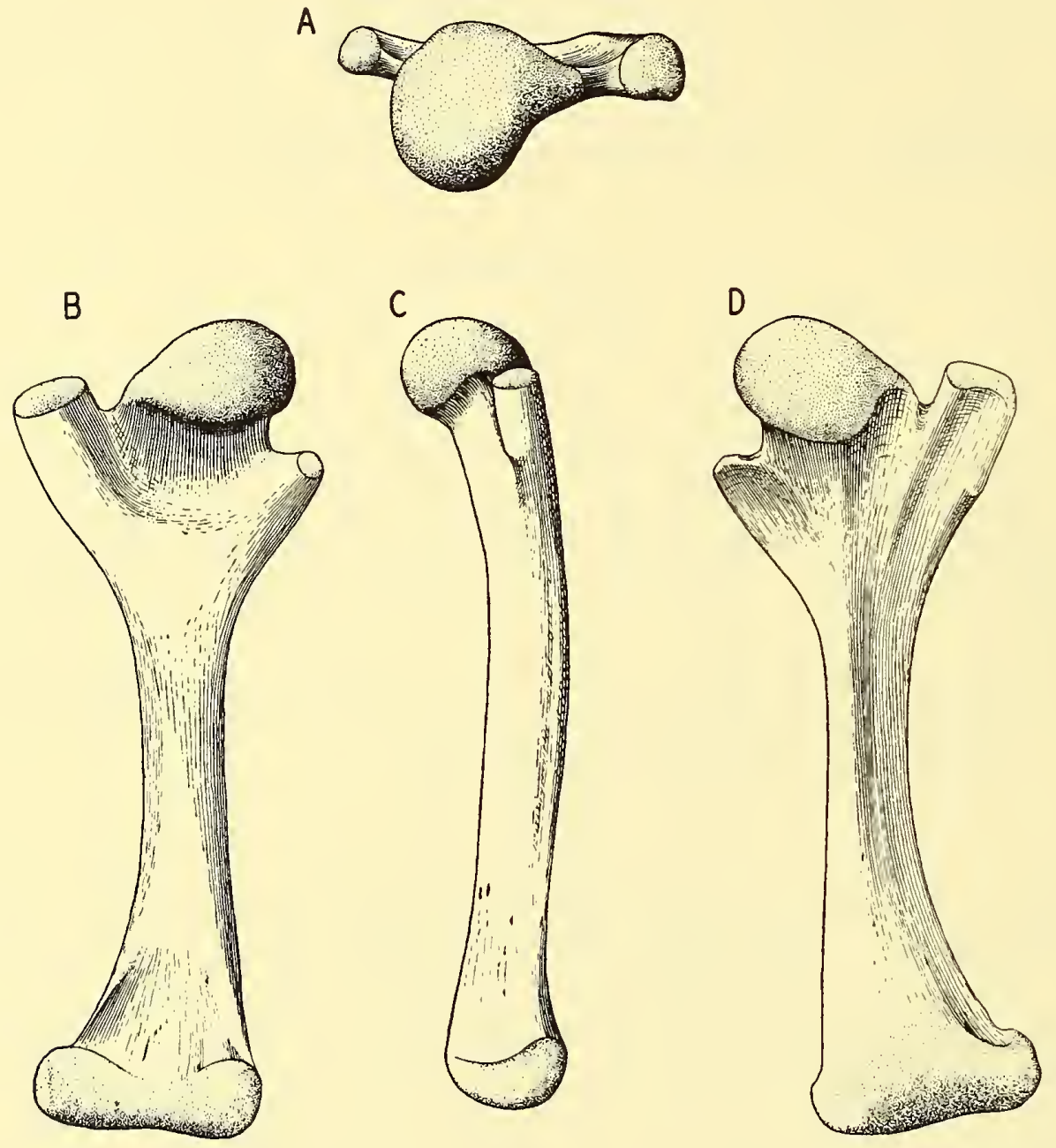

TEXT-FIG. 58.-Oligokyphus, Left femur. $\times$ 2. Based on R.7465, R.7466, R.7467, R.7469, R.7470. A, Proximal view. B, Ventral view. C, Posterior view. D, Dorsal view.

If the Stonesfield femur does not belong to Stereognathus, it belongs to an animal which is at the same evolutionary level as Oligokyphus. Both femora resemble each other more than the femur of any other reptile or mammal, including Bienotherium.

An important sequence of femora is now available: Diademodon sp. (R.227 in Professor D. M. S. Watson's collection, the specimen having been figured and discussed by Romer (I924)), Bienotherium (Young I947), Oligokyphus, the Stonesfield femur, and the femur of Monotremes. The greatest morphological gap is between Diademodon and Bienotherium. The changes involved are the loss of the internal and the fourth trochanters, the separation of the caput and the greater trochanter by a notch, and the development of the lesser 
trochanter from a hardly elevated insertion area on the dorsal side near the anterior margin. In Theria the distance between the caput femoris and the lesser trochanter remains the same as in Bienotherium, but the trochanter migrates to the ventral side of the bone. In Oligokyphus and the Stonesfield femur the distance between the lesser trochanter and the caput decreases and the trochanter remains in its anterior position. In Ornithorhynchus the lesser and the greater trochanters are on the same level and the anterior position of the former is retained. In fact, the lesser trochanter of Oligokyphus and of the Stonesfield femur is less Therian than in Bienotherium and more like Ornithorhynchus.

Five specimens, R.7476-7480, are juvenile, as shown by their size (about $22 \mathrm{~mm}$.), and by the numerous longitudinal grooves, leading to orifices of Haversian canals. R.7478 has the caput preserved and thus demonstrates that the caput was not an epiphysis. Assuredly therefore Oligokyphus did not possess epiphyses on the limb bones.

\section{THE TIBIA}

(Text-fig. 59)

Material: R.748I-7493 and a great number of fragments of the shaft.

Length of the tibia :

R.748I, $36 \cdot 0 \mathrm{~mm}$.

R. $7489,38.8 \mathrm{~mm}$.

R.749I, $42 \cdot 0+c a .6 \cdot 0 \mathrm{~mm}$.

The stout tibia has a large subtriangular and flat proximal surface, articulating with the femur. This surface slopes a little medially and has two faint concavities, the anterior

A

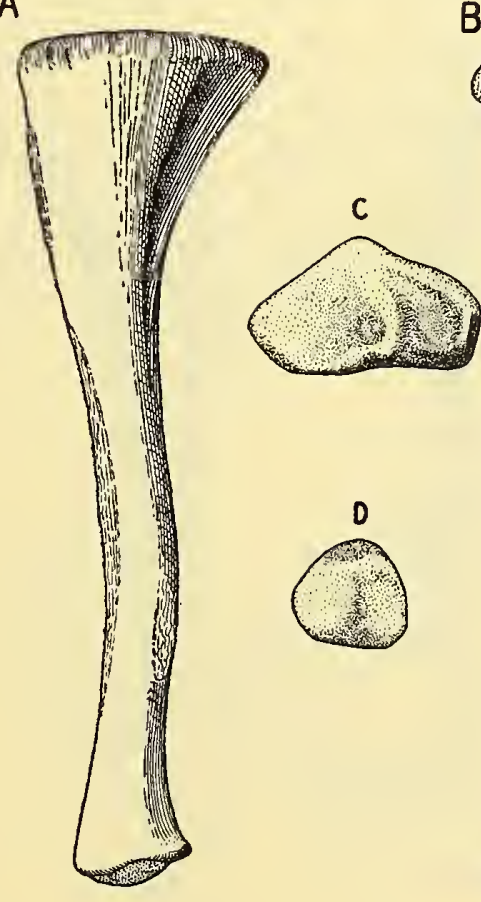

B

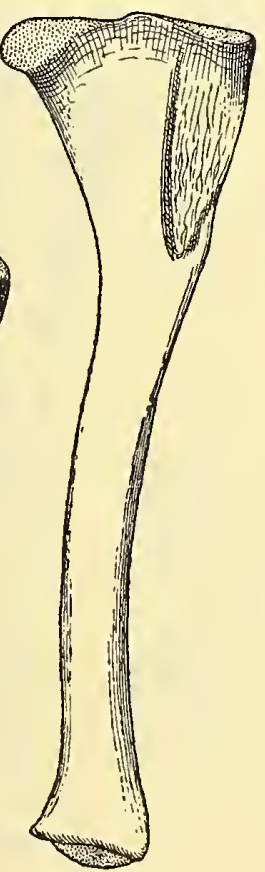

TEXT-FIG. 59.-Oligokyphus. Left tibia. $\times$ 2. Based on R.748I, R.7482, R.7485, R.7487. A, Lateral view. B, Medial view. C, Proximal end. D, Distal end. 
smaller and deeper than the posterior. There is no deep furrow on this surface and there are no pits for cruciate ligaments.

Posterior to the well-developed cnemial crest the shaft is concave, accommodating the M. tibialis anterior. Two-thirds down the shaft on the posterior side is a very pronounced flat rugosity, oval in outline; this is for the interosseous membrane. Antero-laterally there is a long and well-marked rugosity. The medial side of the tibia bears on its proximo-posterior corner the small and vertical articular surface for the fibula. There is a well-defined insertion area for the M. popliteus.

Distally the bone widens abruptly, especially on the posterior side. The distal articular surface is triangular, the posterior moiety is depressed and ends with a sharp edge on the posterior margin, with no distal facet for the fibula. The anterior moiety of the distal surface is well rounded. The curvature of the tibia is the same as in Didelphis.

\section{THE FIBULA}

Material: R.7494-98.

$$
\text { (Text-fig. 60) }
$$

The length of the whole bone in an assembled specimen is $4 \mathrm{I} \mathrm{mm}$.

The few specimens cover the bone completely and a reliable reconstruction is possible. There is one complete proximal end, R.7494, and two well-preserved distal ends, R.7496-7.

The drawings present the anterior and posterior views, which are equivalent to medial and lateral in human osteology. Proximally the fibula is latero-medially expanded; the middle third of the shaft is triangular, the corners of the triangle being medial, lateral and anterior. The proximal end has two rounded oval surfaces devoid of compacta. The
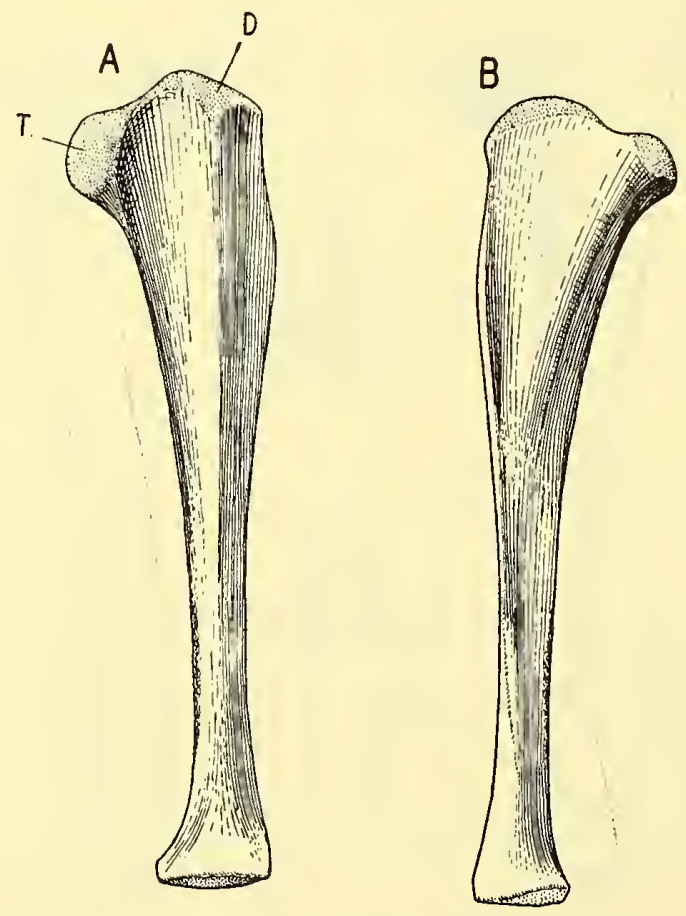

TEXT-FIG. 60.-Oligokyphus. Right fibula. $\times$ 2. Based on R.7494, R.7495, R.7496. A, Anterior view. B, Posterior view. D., Peronecranon; T, Tibial articulation. 
antero-lateral one is for the tibial contact, the postero-medial for M. peroneus. The expanded proximal part has a long concavity on the posterior side, and a long ridge on the middle of the shaft corresponds to it on the anterior surface. Two-thirds down the shaft the lateral edge is slightly widened and rugose, corresponding to the attachment area for the interosseous membrane on the tibia. The distal articular surface is oval and its border with the shaft is rounded. Two faint and shallow concavities are noticeable.

\section{TARSUS [AND CARPUS]}

Apart from the calcaneum and astragalus, there are seven carpal or tarsal bones represented by from eight to three specimens, and six which occur only once in the collection. All these specimens are well ossified and show many definable characters.

In Professor D. M. S. Watson's material of Diademodon there are four carpal or tarsal elements. One, the left calcaneum, agrees well with this element of Oligokyphus. The other three bones show no resemblance to any of the twelve bones in the Oligokyphus collection. No attempt is made to identify these carpal and tarsal bones of Oligokyphus. The carpus and tarsus of Lycaenops (Schaeffer, I94I ; Colbert, I948) and Scymnognathus (Broili \& Schröder, I935) have not been figured so as to make identification possible with the carpal and tarsal bones of Oligokyphus. The hand and foot of "Microgomphodon", a juvenile Diademodon, display perfectly preserved bones, but these are visible only from the dorsal side, which is not helpful for identification of the Oligokyphus material.

\section{THE CALCANEUM}

Material: R.7499-7502.

(Text-fig. 6I)

The calcaneum of Triassic Therapsids is the largest element among the carpal and tarsal bones; it may have a tuber calcis, its medial margin carries the articular facet for the astragalus and its distal margin articulates with the cuboid.

A bone having these characters occurs in the material. In fact, the calcaneum of Oligokyphus is very characteristic and so similar to Diademodon, the Gorgonopsid Hipposaurus and the Bauriamorph Eviciolacerta, that there is no doubt about its identity. In the three
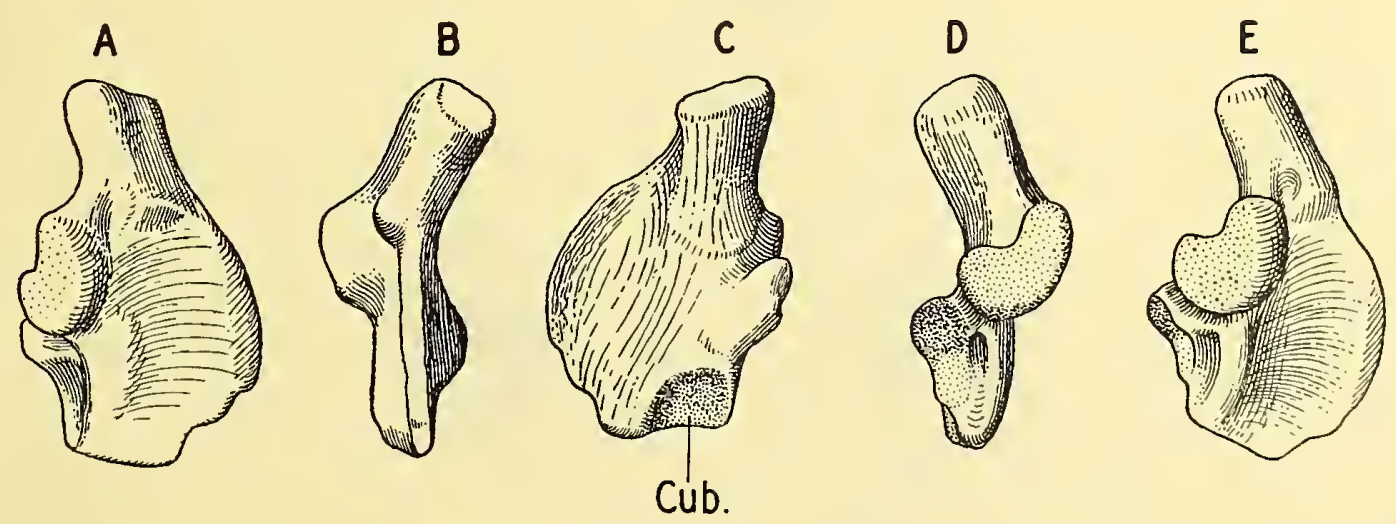

TEXT-FIG. 61.-Oligokyphus. Left calcaneum, R.7500. $\times 3 . \mathrm{A}-\mathrm{D}$, The bone in four positions. E, Anatomically dorsal view. $C u b$., Facet for cuboid. 
genera mentioned, and in Oligokyphus, the tuber calcis is well developed; it is lacking in the Gorgonopsids Lycaenops and Aelurognathus and in Cynognathus.

The calcaneum of Oligokyphus is less mammal-like than in Diademodon or even Hipposaurus. The lateral shelf-so conspicuous in Pelycosaurs-is wider in Oligokyphus than in Diademodon. The sustentaculum is smaller in Oligokyphus than in Diademodon.

The lateral margin of the calcaneum of Oligokyphus is featureless, rounded and thin. The anterior margin has medio-ventrally a deep concave and triangular facet for the cuboid. The medial margin carries the joint fibular and posterior astragalar facet, the sulcus calcanei and the anterior astragalar facet on the sustentaculum. Posteriorly there is a welldeveloped heel. Text-fig. 6I, A shows the calcaneum lying flat on a horizontal plane; $\mathrm{B}, \mathrm{C}$ and $\mathrm{D}$ show it when turned at $90^{\circ}, \mathrm{I} 80^{\circ}$ and $270^{\circ}$. These are not the anatomical dorsal, ventral, lateral and medial positions. If the left astragalus is articulated with the calcaneum the tibial articular surface on the former looks directly medial when in the position of $\mathrm{A}$, Text-fig. 6I, which is impossible. The natural position of the calcaneum when seen from above is given in Text-fig. 6I, E, and, with the astragalus in articulation, in Text-fig. 62, D.
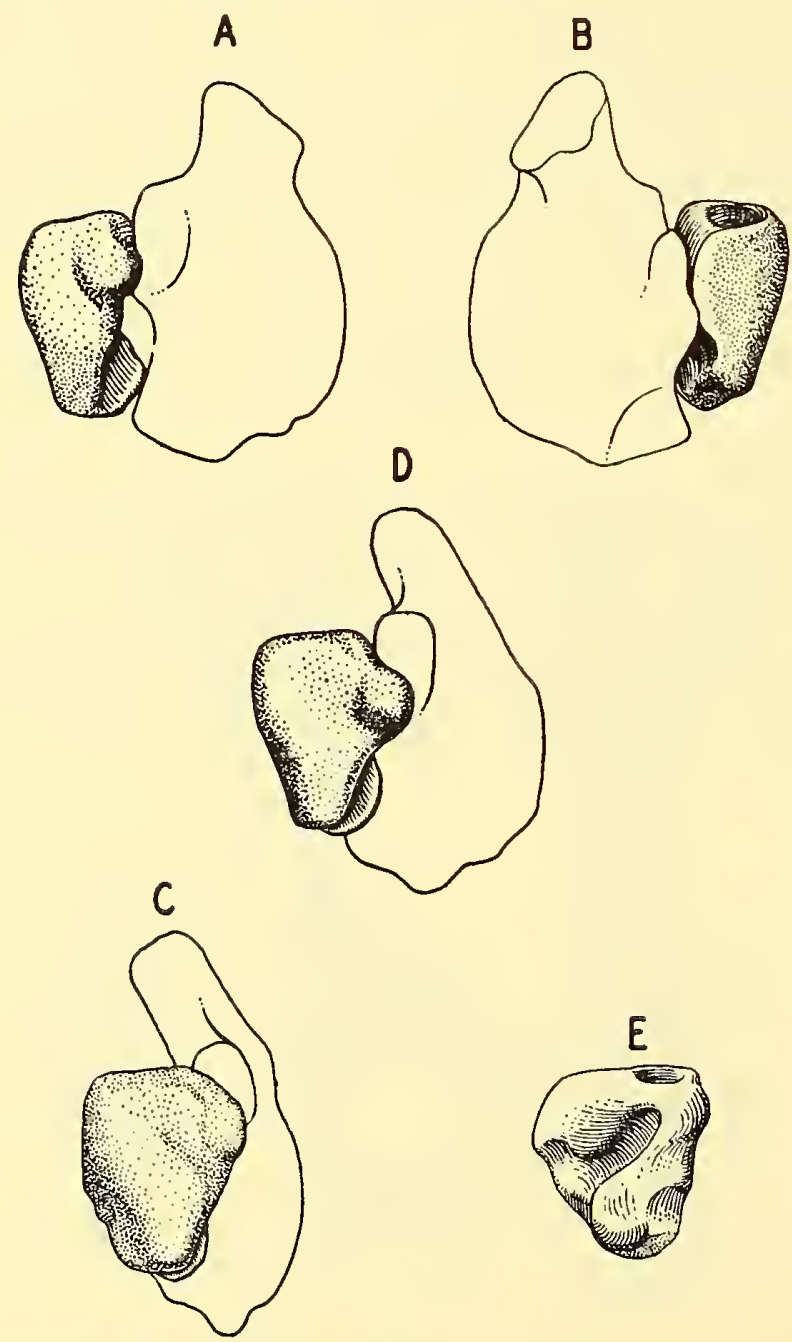

TEXT-FIG. 62.-Oligokyphus. Left astragalus. $\quad \times$ 3. A-D, based on R.7503. E, based on R.7504. $\mathrm{A}, \mathrm{B}, \mathrm{C}, \mathrm{E}$. The bone in four positions. D, Anatomically dorsal view. 
The superposition of the astragalus on the calcaneum in Oligokyphus is realized not so much by the medially extending sustentaculum but by the tilting of the calcaneum in such a way that its medial margin is raised at $30^{\circ}$, taking the astragalus with it. That the postulated position of the two bones is anatomically correct is corroborated by the position of a sulcus on the ventral side between the tuber calcis and the posterior astragalar facet. This sulcus serves for a flexor tendon, possibly for the flexor hallucis longus ; orientating this sulcus normally, the calcaneum is tilted as indicated above.

\section{THE ASTRAGALUS}

Material: R.7503-06.

(Text-fig. 62)

A very primitive Therian astragalus may be characterized as follows : head small and hardly distinct. Uniform, non-trochleated tibial articulation; if ridges are present on it, they extend antero-posteriorly. Sulcus tali dividing anterior from posterior facet for the calcaneum. Sulcus tali beginning at the middle of the lateral margin and ending in an astragalar foramen. Anteriorly on the head there is a facet for the navicular.

This diagnosis covers Oligokyphus. Among primitive Mammalia Didelphis possesses a very similar astragalus but lacks the astragalar foramen. In Eucosmodon, the only Multituberculate in which the astragalus is known, the astragalar head is entirely wanting but the astragalar foramen is present.

\section{THE METAPODIALS}

$$
\text { (Text-figs. 63, 64) }
$$

Material: R.7508-23, also sixty whole specimens or proximal ends and numerous shafts and distal ends.

It can readily be assumed that Oligokyphus had a pentadactyle pes and manus. An even distribution should therefore provide an average of 3.8 specimens of each metapodial. Actually two metapodials have not been found in the material and of two others twelve and eight specimens respectively have been identified. Two reasons might explain the uneven representation of the metapodials: (I) a very uneven representation could be expected on the evidence of the other skeletal elements found in the matrix. However, the eight metapodials found are rather uniform and, assuming that the proportions of the two missing metapodials are similar to the others, this reason would not explain much of the actual discrepancy of the material. (2) Most, but not all, metapodials have been embedded in a complete condition. However, the extrication of these fragile bones was a process demanding the greatest care and concentration in an endeavour to preserve the specimens intact. Only twenty-two whole specimens are available. A minute injury to the proximal end was often irreparable and would deprive the specimen of much of its diagnostic character.

To facilitate reference and future work, the metapodials have been given letters from $a$ to $j$. The metapodials $g$ and $i$ are those still missing. The identification of the metapodials is tentative and articulation of the small bones has not been possible. Their lateral and medial articular surfaces are not so perfect or so well characterized that even two would fit together without any doubt. 


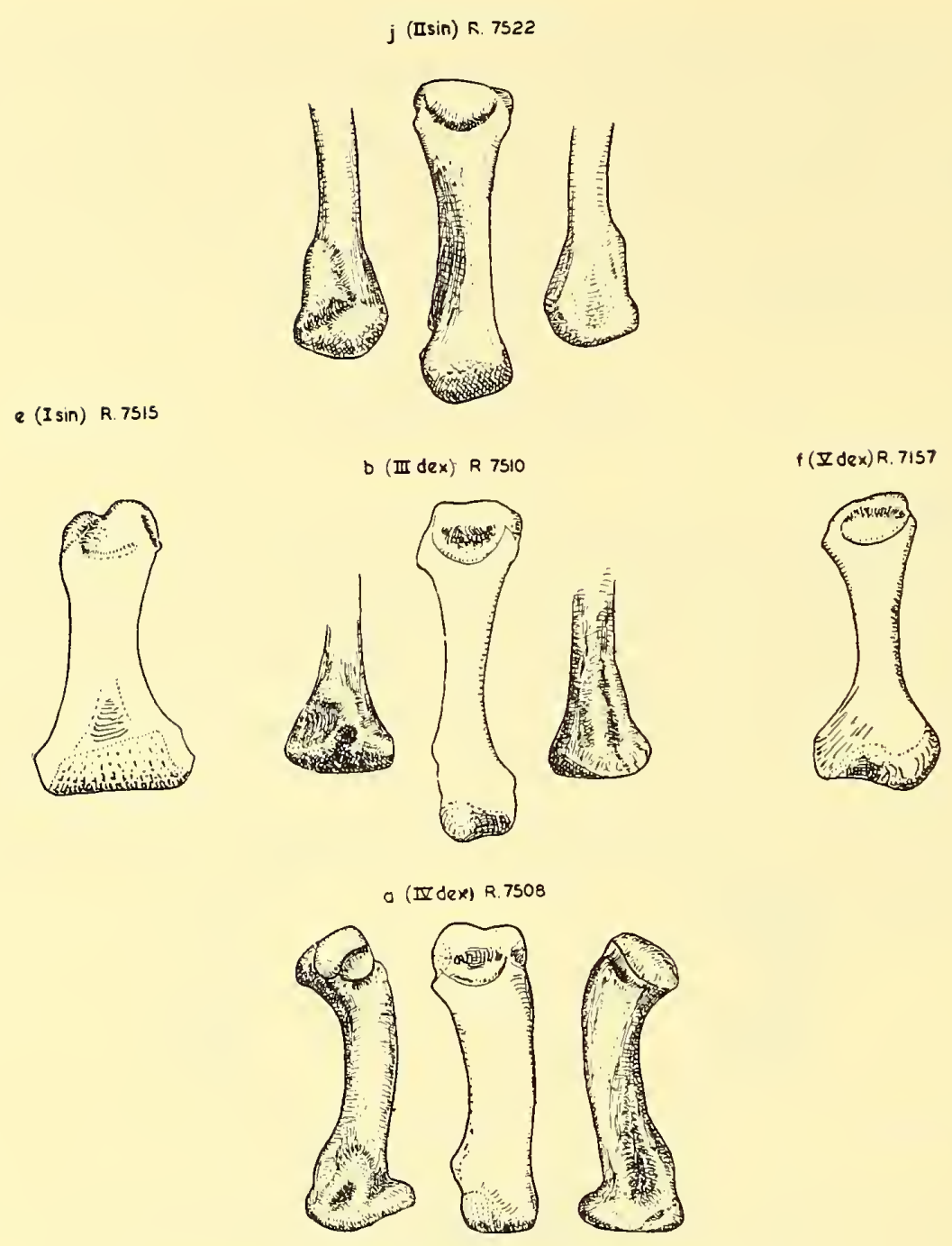

TeXt-Fig. 63.-Oligokyphus. Metacarpals. $\times 4$.

Identification was made on the following assumptions : metapodials I and $\mathrm{V}$ are probably shorter than II-IV and are likely to have laterally and medially expanded proximal ends. The proximal ends of metapodials II-IV are likely to have laterally and medially interlocking surfaces. Metacarpals are likely to be shorter and stouter than metatarsals.

Length of Metapodials of Oligokyphus sp. in mm.

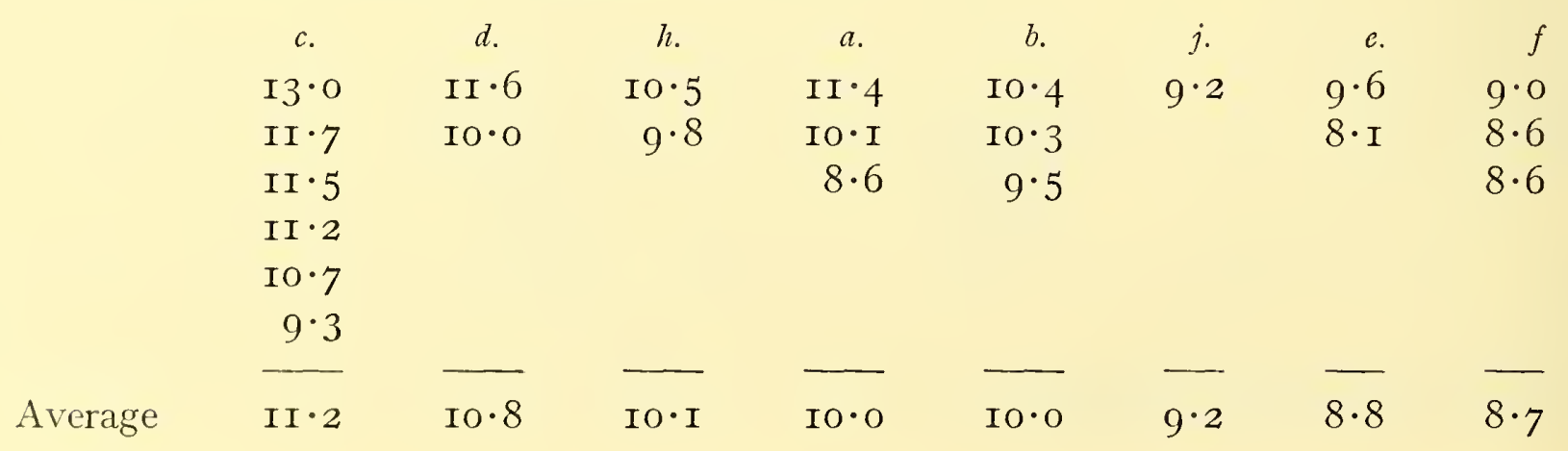


C (III) 8.7512
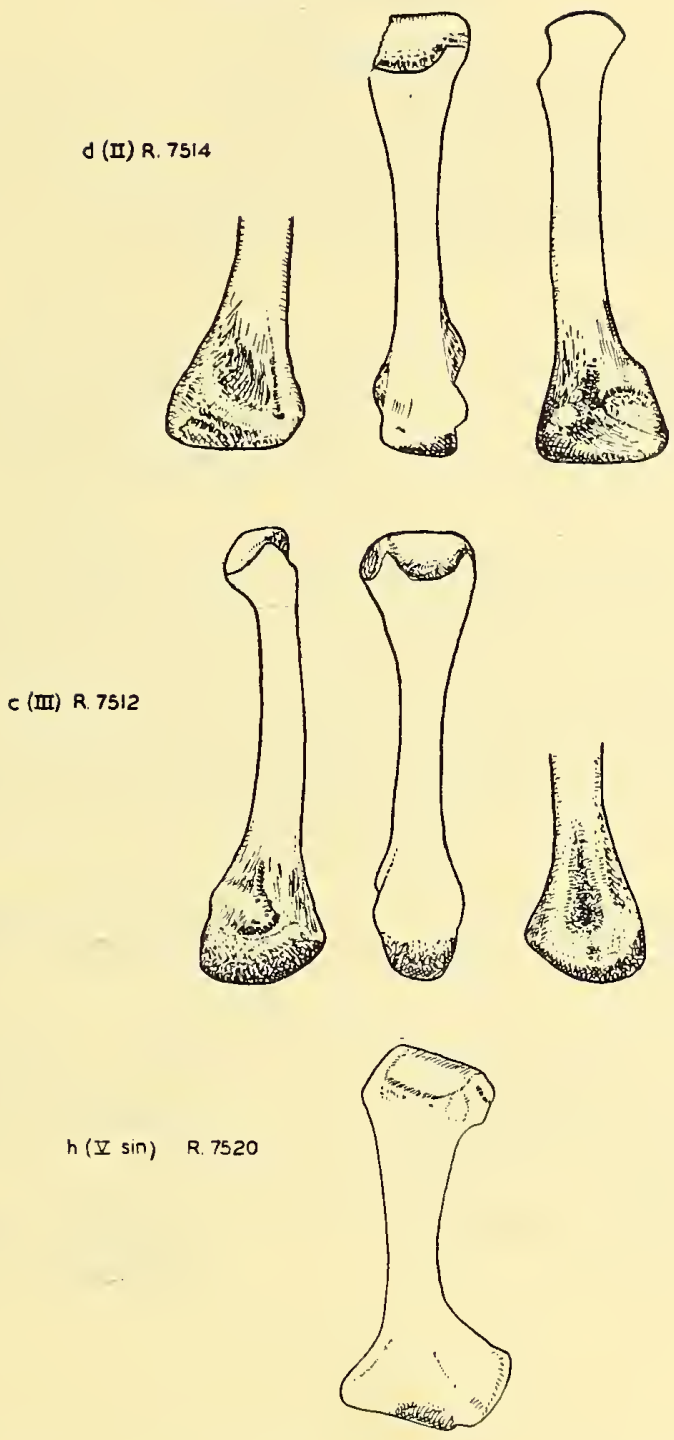

TEXT-FIG. 64.-Oligokyphus. Metatarsals. $\times 4$.

$e, f$ and $h$ are doubtless hallux or pollex or minimus ; $e$ is certainly Mc I; $f$ and $h$ are morphologically very similar, distinguished by features on the ventral side of their proximal end ; $f$ is $\mathrm{McV}, h$ is $\mathrm{MtV} ; c$ and $d$ are certainly metatarsals from II-IV; $a, b$ and $j$ are smaller and from the metacarpus.

The smallest metapodial is $e$. Proximally it has a simple transverse cylindrical joint surface, allowing flexion and extension in one plane. Medially and laterally there are no contact surfaces for carpalia. It is certain that $e$ is the metacarpal of an opposable pollex ; its apparently dorsal side was actually its medial side and its flexors and extensors were actually adductors and abductors. Its distal end is grooved, the groove extending well to the "dorsal " side; the groove divides the distal end asymetrically. The distal ends of the other metapodials are ventrally grooved, but distally and dorsally evenly convex, allowing adduction and abduction of the digits. 


\section{OLIGOKYPHUS}

\section{THE PHALANGES}

(Text-fig. 65, A-H)

Material: R.7524-25 and twenty-five complete specimens and numerous fragments unregistered.

Two distinct types of phalanges can be distinguished by the characters shown below: Average length :

$$
7.9 \mathrm{~mm} \text {. }
$$

Shaft ventrally flat.

Shaft not tapering.

Proximal end concave.
$6.7 \mathrm{~mm}$.

Shaft round in transverse section.

Shaft tapering anteriorly.

Proximal end keeled.

It is clear that the smaller phalanx, being keeled, could not articulate with any metapodial except $e$. It is therefore evident that the large phalanx is the proximal and the small one the distal. The flattening of the ventral surface of the proximal phalanx is due to the presence of an annular ligament for the tendon of the flexor digitorum profundis, which is inserted anteriorly at the lateral and medial edge of the shaft. An annular ligament is absent in the distal phalanx. Some proximal phalanges have their distal articular end set obliquely. The distal ends of both phalanges allow more flexion than extension.

A

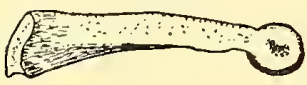

B

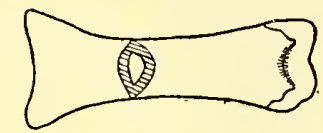

c

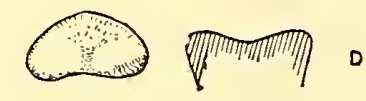

E

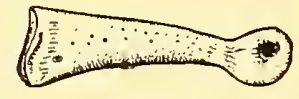

F

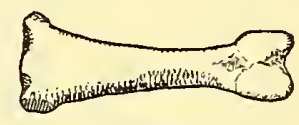

6

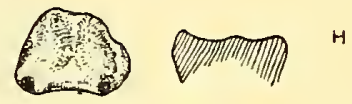

I

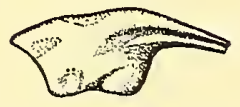

K

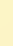

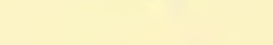

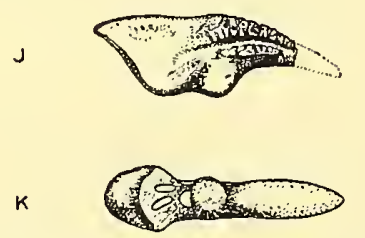

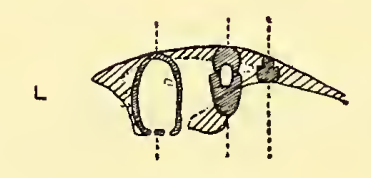

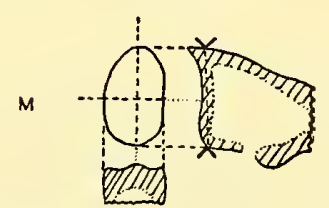

TexT-FIG. 65.-Oligokyphus. Phalanges and claws. $\times 4$. A-D, Proximal phalanx, R.7524: A, Side view. B, Dorsal view. C, View of proximal end. D, Horizontal section through proximal end. E-H, Distal phalanx, R.7525 : E, Side view. F, Ventral view. G, View of proximal end. H, Horizontal section through proximal end. I-M, Claws : I, Side view of R.7526. J, Side view of R.7527. K, Ventral view of R.7528. L, Vertical sagittal section of claw, with three transverse sections inset. M, Outline of proximal end of claw, and below, horizontal section through proximal end. 


\section{THE CLAW}

(Text-fig. 65, I-M)

Material: R.7526-28 and thirty unregistered specimens.

In most mammalian claws the proximal part is much shorter than in Oligokyphus. The claw of Varanus equals that of Oligokyphus. The pointed tip of the claw is laterally grooved and ventrally flattened.

It is easy to show, by a process of elimination, that the claw of Oligokyphus has no relationship with claws of animals with " extreme" habits : for instance, with the nail or the hooklike claw of the slow and determined quadrupedal climber, with an opposable pollex and hallux and often without a tail ; or with the nail or hook of the brachiator; or the strongly compressed and high claw of the arboreal jumper and runner, which always has a long tail and which may lack opposition of the first digit ; or finally, with the split claw or the depressed and spatulate claw of the digger.

The whole of the skeleton of Oligokyphus gives the impression of a rather slow animal, strongly built for its size. There is little elongation of the hind extremity Pes and manus are plantigrade. Extension of the phalanges took place while the limb was not bearing weight, at the beginning of the forward movement of the limb, before it lost touch with the ground.

The metacarpus and metatarsus are strong, rather short and spreading. The pollex is opposable. The digits allow abduction and adduction. Some distal phalanges are set at an angle to their proximal one. The distal phalanx is very little shorter than the proximal one. This character is essential, and excludes wholly terrestrial habits of Oligokyphus; all terrestrial mammals have the distal phalanx much shorter than the proximal one.

It can be said in conclusion that Oligokyphus was more arboreal than terrestrial, but it could dig as well as swim, and run as well as climb, if the occasion demanded it. 


\title{
SYSTEMATIC RELATIONSHIP OF OLIGOKYPHUS
}

\author{
THE FAMILY TRITYLODONTIDAE
}

Bienotherium, Tritylodon, Oligokyphus and Stereognathus are so very much alike that they are easily accommodated in one family, the Tritylodontidae. The occlusion of the cheek teeth is identical in all of them. The number of cusps in the upper cheek teeth varies little being 8 in Bienotherium, Io in Tritylodon, II in Oligokyphus and 6 in Stereognathus. Only in Tritylodon and Oligokyphus are the cheek teeth well enough preserved for the enumeration of small cusps. The number of upper cheek teeth is 7 , except in Oligokyphus minor and possibly Stereognathus where it is 6 . The number of upper incisors is 3 in Tritylodon, Oligokyphus and Bienotherium. Though Young counts only two upper incisors in the last named, there was probably a small symphyseal incisor, medially to the large $I^{2}$. Though it has been lost in the type of Bienotherium yunnanense there is doubtless a space for it. The structural differences in the cranium are due either to different size and proportions (thus the snout is broad in Bienotherium, elongated in Tritylodon and Oligokyphus) or to the considerable difference in geological age between the various members of the family. The palate is far deeper below the basicranial axis in Oligokyphus than in Bienotherium; the lesser trochanter of Bienotherium is in a more Therian position ; and the lesser trochanter of Oligokyphus approaches the position it has in the Monotremes. No Tritylodontid younger than Stereognathus is known and the family became extinct before the Purbeckian.

\section{THE TRITYLODONTIDAE AND THE ICTIDOSAURIA}

The evidence for including the family Tritylodontidae in the Sub-order Ictidosauria was set out by Parrington (I947) who states that Tritheledon (in so far as its parts are known), Broom's Ictidosaurs $\mathrm{A}$ and $\mathrm{B}$, and the Tritylodontidae have the following characters in common: (I) prefrontal and postorbital bones lost, (2) postdentary bones greatly reduced, (3) dentary deep with a groove and ridge for the support of the postdentary bones and a boss against the pterygoid flange, (4) initial elaboration of the tooth-crowns transverse to the length of the jaws, (5) subtemporal fossae enlarged anteriorly and posterior cheek teeth withdrawn on to the palate between them.

The exclusion from this Sub-order of Karroomys, the American Protodonta Dromatherium and Microconodon, Lycorhinus, Pachygenelus and Kunminia is indicated because (4) and (5) do not apply to them. Until the sub-order Ictidosauria can be better defined, it is not advisable to put into it any further genera which are based on teeth alone. This applies to the Microlestidae.

\section{THE TRITYLODONTIDAE AND THE MICROLESTIDAE}

Microlestid cheek teeth and Tritylodontid lower cheek teeth have some salient features in common. Both families may be of a similar grade but little is gained by speculating on their relationship while our knowledge of the Microlestidae is based entirely on isolated teeth.

An upper Tritylodontid cheek tooth is in all respects a replica of a lower cheek tooth turned 
$\mathrm{I} 80^{\circ}$ and provided with an additional row of cusps. We have already stated that the internal row of cusps is the most variable of the three. If higher variability is regarded as a feature of a dental structure less ancient and less well established than its more conservative older neighbours, one could maintain that the internal row of cusps in the upper cheek teeth is a neomorph and that the ancestors of the Tritylodontidae had upper and lower cheek teeth that were identical, except that the slope of the row of cusps and the crescent faces of each cusp point in opposite directions in the upper and lower jaws.

A condition tentatively suggested by Parrington (1947: 720), when discussing the teeth of Microlestes has probably been realized in the cheek teeth of the contemporary Tritylodontidae. Parrington writes: "The comparison of the front of an upper tooth with the back of a lower tooth seems reasonable if the teeth are regarded as serially homologous units developed from denticles which were originally arranged along a gill arch and reached their present position by the folding of the latter when it was formed into jaws." Although this proposed comparison or homology of an anterior element of the tooth of the lower dentition with a posterior element of a tooth of the upper dentition is not, according to Parrington, met in Microlestes, it may nevertheless hold for the cheek teeth of that genus.

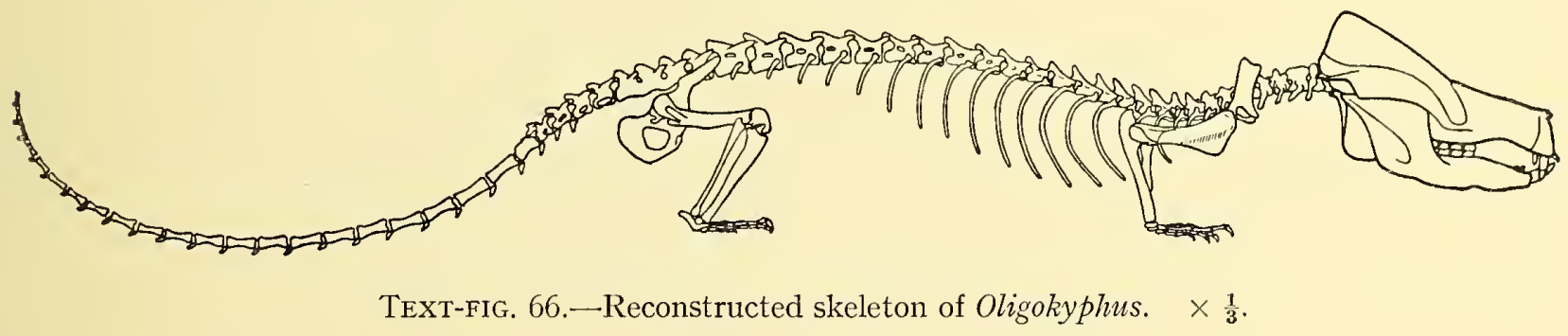

The required homology cannot arise in a haplodont reptilian dentition because the functional upper and lower teeth must either be curved backward or be straight. The same applies to carnivores which have a differentiated dentition, for instance most Cynodonts. The Gomphodonts cannot be considered because they have not yet developed tooth characters which allow homologization. The earliest mammal-like dentition which does allow it, the Tritylodontid cheek tooth dentition, shows the required homology and this evidence ought to be of some weight.

\section{POSSIBLE ANCESTORS OF THE TRITYLODONTIDAE}

Of Triassic Therapsids which could be ancestral to the Tritylodontids, only the Cynodonts and Bauriamorphs need be discussed. The Bauriamorphs pass into the Cynognathuszone and the Cynodonts are known from the Stormberg beds. The greater abundance of Cynodont and Tritylodont material, in comparison with the rare Bauriamorphs, of which only one skeleton is known, produces a relatively greater number of common characters and thus might lead to a bias in the estimation of the relationships between the groups. The relationship, however, of Tritylodontids to Cynodonts is actually much closer than to Bauriamorphs, even when skulls alone are considered.

The presence of an interpterygoid vacuity and sub-orbital fenestra, and the absence of a large anterior palatal foramen characterise Bauriamorphs but not the contemporary 
Cynodonts and Tritylodontids. No known Bauriamorph is structurally so close to the Tritylodontidae as are the Cynodonts. Even if future discoveries were to reveal late Triassic Bauriamorphs which had lost the postorbital bar and had posterior upper cheek teeth extending back in the temporal fossa, they would still not be probable predecessors of Tritylodontids.

Broom (I932: 3I4, 33I) considered it likely that the Ictidosaurs were derived from small Bauriamorphs. But this opinion was formed before the new finds of Tritylodontids were made and before it became certain that they were Ictidosaurs. Romer (I945) does not commit himself as to the origins of the Ictidosauria. Watson (I942) has no doubt that the Tritylodontids are phylogenetically related to Gomphodont Cynodonts. Parrington (I947) implies a phylogenetic relation between Cynodonts and Ictidosaurs.

The present writer agrees with Parrington and Watson, for the Cynodont cranium allows a direct derivation of the Tritylodontid. The appendicular skeleton of Cynodonts also is built on the same plan as that of Tritylodontids. The only Cynodont character which has been found to be specialized and which occurs in neither the Tritylodontids nor the predecessors of the Cynodonts - the Gorgonopsids-is the very peculiar, widened, lumbar ribs that are fused with the vertebrae. Specializations present in geologically older forms and absent in geologically younger ones seem to preclude the former from the ancestry of the latter though Gregory (I947 : 45) contests this dictum. None the less it may be wiser to look for the ancestor of the Tritylodontidae among still unknown Cynodonts which lacked the widened lumbar ribs than to assume that this specialization was developed during the transition from Gorgonopsids to Cynodonts, and lost during the transition from Cynodonts to Tritylodontids. The fact that all known Cynodonts have broadened lumbar ribs does not exclude the possibility that some Cynodonts may have lacked them.

Though the osteological analysis makes it clear that Oligokyphus and all other Tritylodontidae are derived from a Gomphodont Cynodont, no more precise statement is as yet possible. Tritheledon, which is contemporaneous with the Stormberg Tritylodontids, is of no assistance in this problem.

\section{THE TRITYLODONTIDAE AND THE MULTITUBERCULATA}

Are the Tritylodontidae phylogenetically related to the Multituberculata sensu stricto (i.e. excluding the Tritylodontidae and all Triassic and Rhaeto-Liassic genera) ? Since Tritylodontids became known, their relationship with Upper Jurassic Multituberculates has not always been taken for granted. Seeley (I895) regarded Tritylodon as intermediate between mammals and reptiles. Woodward (1898) regarded Tritylodon as a Theriodont, but put Stereognathus among the Multituberculates with reservation. In I925, however, Woodward placed Tritylodon among the Multituberculata.

After Bienotherium became known, Watson (I942 : Io9) commented on this problem, but did not commit himself. He said that a widening of the posterior part of the dentary of Bienotherium would bring this bone into contact with the squamosal, making an articulation similar to that in Ptilodus and Taeniolabis. Such a statement, from such an authority, might be used by those eagerly seeking for phylogenetic relations among forms still very little known. However, a few lines farther on Watson states " . . . it seems to me probable that the group [The Ictidosauria] as a whole consisted of highly-specialized terminal forms and left no descendants." 
Simpson at first (I928) included the Tritylodontids in the Multituberculates, but in 1937 and 1938 he no longer maintained this relationship. In I943 the writer expressed his opinion in unmistakable terms-an opinion still held-that Tritylodontids and the Multituberculates are phylogenetically unrelated, the earliest known Multituberculates being those from the Purbeck-Morrison.

Bohlin (I946) and Simpson (I945) agree with this view. If accepted, this means that we do not know whence Multituberculates may have sprung. Hennig (I947), among others, dissents, and still upholds in principle his classification of I922. A substantiation of the opinion, which is in accord with the quotation from Watson and with Simpson and Bohlin, is thus necessary. The pertinent genera in this discussion are evidently the Tritylodontids, and Plagiaulax and Ctenacodon. Four characters must be mentioned: (I) the Tritylodontid upper cheek teeth have three longitudinal rows of cusps, the lower teeth have two. In the sequence of Tritylodontids from the Upper Triassic to the Bathonian there is not one exception to this. As for Mucrotherium and Uniserium see Kühne (I950a). Are we to believe that from the Bathonian to the Purbeckian this rigidly maintained occlusal pattern is changed in the Plagiaulacidae and reintroduced in their successors the Ptilodontidae? (2) The crescentic cusp of the cheek teeth is a diagnostic character of the Tritylodontids. Are we to believe that after thirty million years this character changes, giving way to a more or less conical cusp in Plagiaulax and Ctenacodon, to be redeveloped among the Tertiary Multituberculates? (3) In contrast to the Ictidosauria A and B, the Protodonta and the Cynodonts, the Tritylodontidae have a cheek tooth dentition of uniform elements. The difference of the cheek teeth is numerical only, they contain a greater or a smaller number of cusps. The difference is not one which goes with different function of different elements of the cheek tooth dentition. Are we to believe that this kind of dentition gives rise to the heterodont cheek tooth dentition of the Plagiaulacidae? (4) From Bienotherium to Oligokyphus the basicranial axis rises higher and higher above the palatal axis. The two Multituberculates of which we know the skull-Taeniolabis and Ptilodus-have the two axes in line, as have all primitive mammals. Are we to believe that this trend reverted to an original stage while the stock continued to exist? Assuming a phylogeny such as Hennig propounds, there would be a four-fold reversal and a two-fold reintroduction of trends from the Tritylodontidae to the Ptilodontidae.

\section{THE TRITYLODONTIDAE AND THE MONOTREMES}

The skulls of both groups are so extremely specialized that there is little point in trying to find specific characters in common. The fact that a Liassic Therapsid has both shoulder and pelvic girdles closely similar to those of Monotremes is a further argument supporting those who regard the Monotremes as mammals evolved, independently and in isolation, from a very early period of mammalian history, and that (Simpson, I945: I68) "their combination of extreme specialization in habitus characters with the retention of some very primitive and even reptilian heritage characters makes the monotremes entirely isolated among recent mammals".

If Gregory's paper "The Monotremes and the Palimpsest Theory" (I947) had no other effect than to make one especially cautious, it would still have served a useful purpose. It may be doubted whether Gregory would have written and concluded the paper as he did had he been familiar with the postcranial skeleton of Oligokyphus. 


\section{THE ORIGIN OF THE MAMMALS}

The systematic position of the genus Oligokyphus depends on the presence or absence of an exceptionally important character-complex, the mammalian middle ear and jaw articulation, which for diagnostic value stands far above a number of minor skeletal characters distinguishing Therapsids from mammals. The manifestation of this character-complex in the dentary, a bone which is usually well-preserved when the minute and intricate middle ear is not, is unambiguous, and in this respect Oligokyphus is a Therapsid reptile.

Among the welter of Therapsids from the Lower Permian to the Upper Triassic there are genera which become more and more mammal-like without becoming specialised; they preserve the potentiality of producing mammalian descendants. A sequence of such genera can be assumed but cannot yet be demonstrated by specimens. Members of this sequence were ecologically unobtrusive and played a role comparable to that of the Insectivores in the Upper Cretaceous and Paleocene.

In the Rhaetic the Therapsid stock was depleted; large and powerful forms had died out. The known survivors include small forms like Broom's Ictidosaurs A and B, the Tritylodontidae and the Microlestidae. At this time the most essential and crucial skeletal character, the mammalian middle ear, was evolved, yet this did not lead to any noticeable radiation into many diverse habitats. The terms of a phylogenetic sequence beginning with an early Permian Therapsid and ending with a Middle Cretaceous Proplacental cannot yet be stated. Diademodon is too large and is an omnivorous animal; Oligokyphus is a herbivore and actually contemporaneous with the earliest mammals or immediate mammal ancestors such as Microconodon or Morganucodon. The Monotremes are relics of a Prototherian grade of Therapsid evolution in the Lower Jurassic.

Below are listed mammalian characters which are non-adaptive in the sense that they are not necessary for the possessor's peculiar modes of life. They are those which distinguish Therapsids of different age and they are those which undergo a change in the postulated sequence. The possessor of such characters would be a fitter animal than one without them. The simpler such a character, the earlier it occurs among the Therapsids and the more widespread it is. The structural advance towards a mammalian condition is maintained-as far as the skeleton is concerned--by reduction, elaboration and differentiation. For instance, the establishment of the mammalian digital formula, the loss of the postorbital bar and many changes in the shoulder-girdle, are reductions. The formation of a free acromion, of the supraspinous part of the scapula, the formation of the tuber calcis and of the olecranon are elaborations. The establishment of a heterodont dentition and the individualization of the vertebrae are differentiations. The same functions will be performed by many elements at an early stage and by a few elements at a later stage, and efficiency will be gained. Where one element is performing the same function at an early and a late stage, it will do it at the later stage more effectively because of its elaboration. Where many similar elements are present (polyisomeres) at an early stage and no reduction takes place at the later stage, differentiation of those elements will take place. 


\section{Mammalian Skeletal Characters}

I. Characters occurring in pre-Molteno Therapsids: (I) olecranon; (2) tuber calcis; (3) plate-like epipterygoid ; (4) secondary palate ; (5) double occipital condyle ; (6) loss of pineal foramen ; (7) loss of postorbital bar ; (8) loss of ectepicondylar foramen ; (9) free acromion; (ro) digital formula 23333.

(Characters I-5 are found in Diademodon.)

II. Characters that occur for the first time in Oligokyphus or other Tritylodontids: (r) cheek teeth with more than one root; (2) internal auditory meatus ; (3) loss of prefrontal and postorbital; (4) tangential thoracic vertebrae; (5) platycoelous vertebrae; (6) dens epistrophei; (7) cervical ribs fused with vertebrae; (8) ilium preacetabular; (9) cotyloid notch present.

III. Additional characters found in Monotremes: (I) mammalian middle ear and jaw articulation ; (2) epiphyses ; (3) cerebral elaboration ; (4) atlantal elements fused.

IV. Additional characters found in Theria : (I) tribosphenic molars ; (2) liberation of fore limb with (a) loss of precoracoid, (b) partial loss of coracoid, (c) loss of interclavicle, (d) development of supraspinous part of scapula.

Future discoveries may put many characters now listed under II among those listed under I. In the flourishing Therapsid complex there are many mammalian characters, yet the best known and latest pre-Molteno Therapsid-Diademodon-has only five out of ten of the mammalian characters known to that age (and Cynognathus has only four, as it lacks the tuber calcis). Oligokyphus has all the characters listed under I, although the loss of the pineal foramen is merely inferred. The Monotremes do not lack any character listed under I, but from those listed under II they lack Nos. 7 and 9. There is no suggestion that the Theria have a common ancestor with the living Monotremes later than the Rhaetic.

Relatively early creatures which show one or more mammalian characters may have led to the assumption that actual Theria lived, say, in the Lower Triassic. The strikingly mammalian arm Theriodesmus is a pertinent example. But one mammalian character does not make a mammal. Apart from the necessity of knowing the whole skeleton of an animal in order to assess its mammalian nature, we can only emphasize that some truly mammalian characters came into existence very early and others late. How else could one visualize the evolution from early Permian Pelycosaur-like Therapsids to Middle Cretaceous Theria? Thus the glimpses of mammalian " precursors " are nothing but partial achievements of the Therian grade. These precocious advances are only partial and not correlated with equal advances of the rest of the animal.

Oligokyphus was coexistent with Theria-if we are allowed to infer their presence from the isolated teeth in the Rhaetic of Holwell, Hallau and South Glamorgan-which are likely to possess epiphyses, the mammalian middle ear and jaw articulation, as well as the mammalian arm- and shoulder-construction. But for these teeth from the Rhaetic some observers might conclude that the Theria came into existence considerably later than the Lower Lias.

Being a mammal is a condition which does not depend on a few "diagnostic " characters, but on the co-ordination of such physical and physiological manifestations as we know in 
I 42

OLIGOKYPHUS

mammals. A mammalian locomotor apparatus without a mammalian circulation, nutritron, perception and temperature control is an important step in the direction of the Theria but it does not make its possessor a mammal.

The stages which led from an early Permian Therapsid to a Middle Cretaceous mammal have been minute. The attainment of mammalian status is gradual, not sudden. The evidence available at present is not nearly sufficient to define the phylogenetic line of mammalian descent. Gorgonopsids, Cynodonts and Ictidosaurs as known today are not ancestral to mammals, but they show how the mammals evolved. Each suborder mentioned is more mammal-like than the preceding one. The Ictidosaurs represented by the Tritylodontid Oligokyphus have attained Prototherian status in their postcranial skeleton.

The transition from the class Reptilia to the class Mammalia took place in the Rhaetic. The morphological changes involved in the evolution of the mammalian middle ear and jaw articulation were essential ; to bring it about, many correlated skeletal changes had to occur ; it is probable therefore that the transition took place once only. In my opinion the Monotremes did not evolve independently from the rest of the mammals. 


\section{REFERENCES}

ABEL, O. I9I2. Grundzüge der Paläobiologie der Wirbeltiere. $\mathrm{xv}+708$ pp., I pl. Stuttgart.

BENSLEY, B. A. Ig02. On the identification of Meckelian and Mylohyoid Grooves in the Jaws of Mesozoic and Recent Mammalia. Univ. Toronto Stud. biol., 3 : 75-8I, pl. I.

BÖKER, H. I935. Einfiihnung in die vergleichende biologische Anatomie der Wirbeltiere, 1. xi +228 pp., 225 figs. Jena.

BOHLIN, B. I946. The Jurassic Mammals and the Origin of the Mammalian Molar Teeth. Bull. geol. Instn Univ. Upsala, $31: 363-388$, Io figs.

BROILI, F. \& SCHRÖDER, J. I935. Beobachtungen an Wirbeltieren der Karrooformation, IX. Über den Schädel von Gomphognathus Seeley. S. B. bayer. Akad. Wiss., München, 1935 : II5-I82, pls. I, 2.

- I936. Beobachtungen an Wirbeltieren der Karrooformation, XVIII. Über Cynodontier-Wirbel. S.B. bayer. Akad. Wiss., München, 1936:6I-76, pl. I, fig. 2.

- I I $936 a$. Beobachtungen an Wirbeltieren der Karrooformation, XIX. Ein neuer Fund von Tritylodon Owen. S.B. bayer. Akad. Wiss., München, 1936 : I87-228, pls. I, 2.

BROOM, R. Ig03. On the Axis, Atlas, and Proatlas in the Higher Theriodonts. Proc. Zool. Soc. London, 1903 : I77-I80, pl. I8.

I904. On the Structure of the Theriodont Mandible, and on its Mode of Articulation with the Skull. Proc. Zool. Soc. London, 1904: 490-498, pl. 35.

I905. On the Affinities of Tritylodon. Trans. S.Afr. Phil. Soc., Cape Town, 16: 73-77.

I907. The Origin of Mammal-like Reptiles. Proc. Zool. Soc. London, 1907 : I047-Io61.

I9I0. On Tritylodon and the Relationships of the Multituberculata. Proc. Zool. Soc. London, 1910 : $760-768$, figs. $67,68$.

- - I9I2. On a New Type of Cynodont from the Stormberg. Ann. S. Afr. Mus., Cape Town, $7: 334-336$, pl. 22.

I929. On some Recent New Light on the Origin of Mammals. Proc. Linn. Soc. N.S.W., Sydney, 54 : 688-694, 7 figs.

- I932. The Mammal-like Reptiles of South Africa. xvi +376 pp., III figs. London.

BUTLER, P. M. I939. The Post-Canine Teeth of Tritylodon longaevus Owen. Ann. Mag. Nat. Hist., London (II) $4: 5$ I $4-520,3$ figs.

COLBERT, E. H., I948. The Mammal-like Reptile Lycaenops. Bull. Amer. Mus. Nat. Hist., New York, $89: 353-404$, pls. I-8.

DENKER, A. IgoI. Zur Anatomie des Gehörorgans der Monotremata. In Semon, R. Zoologische Forschungsreisen in Australien und dem Malayischen Archipel III. Denkschr. med.-naturw. Ges. Jena, $6: 667-772$, pls. 21,22 .

DIETRICH, W. O. I937. Uber eine Säugetierelle aus dem Rhät von Halberstadt. N. Jb. Min. Geol. Paläont., Stuttgart (Beil. Bd.) 77B : 310-3I9, pl. 2I.

GRANGER, W. \& SIMPSON, G. G. I929. A Revision of the Tertiary Multituberculata. Bull. Amer. Muss. Nat. Hist., New York, 56 : 6oI-674, 43 figs.

GREgory, W. K. I9Io. The Orders of Mammals. Bull. Amer. Mus. Nat. Hist., New York, $27: 524$ pp., 32 figs.

—. I947. The Monotremes and the Palimpsest Theory. Bull. Amer. Mus. Nat. Hist., New York, 88: I-52, pls. I, 2.

GREGORY, W. K. \& CAMP, C. L. IgI8. Studies in Comparative Myology and Osteology, 3. Bull. Amer. Mus. Nat. Hist., New York, $38: 447-563$, pls. 39-50.

HAUGHTON, B. A. I924. The Fauna and Stratigraphy of the Stormberg Series. Ann. S. Afr. Mus., Cape Town, $12: 323-498$.

HENNIG, E. I922. Die Säugerzähne des württembergischen Rhät-Lias-Bonebeds. N. Jb. Min. Geol. Paläont., Stuttgart (Beil. Bd.) 46 : $18 \mathrm{I}-267$, pls. $2-5$.

- I947. Der Übergang vom Saurier zum Säuger. Naturwissenschaften, Berlin, 34 : 246-249.

HUENE, E. voN. I933. Zur Kenntnis des Württembergischen Rätbone-beds mit Zahnfunden neuer Säuger und säugerähnlicher Reptilien. Jh. Ver. vaterl. Naturk. Wïrttemb., Stuttgart, 89: 65-I28, pls. I-3.

JONES, O. T. I93I. Some Episodes in the Geological History of the Bristol Channel Region. Rep. Brit. Ass., London, $1930: 57-82$, pls. I, 2.

KRÜGER, W. I927. Musculus multifidus und Musculi rotatores der Haussäugetiere und ihre Beziehungen zur Drehfähigkeit der Wirbelsäule. Anat. Anz., Jena, $63: 305-327,2$ figs.

KÜHNE, W. G. I943. The Dentary of Tritylodon and the Systematic Position of the Tritylodontidae. Ann. Mag. Nat. Hist., London (II) $10: 589-601,2$ figs. 
KÜHNE, W. G. I949. On a Triconodont tooth of a new pattern from a Fissure-filling in South Glamorgan. Proc. Zool. Soc. London, $119: 345^{-350,} 2$ figs.

I950. Mucrotherium und Uniserium E.v.Huene sind Fragmente unterer Backenzähne eines Tritylodontiers. Neues. Jb. Geol. Palaont., Stuttgart, 1950 Monatsh. : I87-I9I, 3 figs.

-.. I950a. A Symmetrodont Tooth from the Rhaeto-Lias. Nature, London, 166 : 696-697, fig.

MATTHEW, W. D. rgog. The Carnivora and Insectivora of the Bridger Basin, Middle Eocene. Mem. Amer. Mus. Nat. Hist., New York, $9: 29 \mathrm{I}-567$, pls. 42-5I.

McKAY, W. J. S. I895. The Morphology of the Muscles of the Shoulder-Girdle in Monotremes. Proc. Limn. Soc. N.S.W., Sydney (2) $9: 263-360$, pls. 20-23.

MOORE, C, I865. On the Geology of the South-west of England. Rep. Brit. Assoc. Adv. Sci. London, 1864. Notes \& Abstracts, p. 59 .

OLSON, E. C. I944. Origin of Mammals based upon cranial Morphology of the Therapsid Suborders. Spec. Pap. Geol. Soc. Amer., Washington, 55 : I-I36, 35 figs.

OWEN, R. I87I. Monograph of the Fossil Mammalia of the Mesozoic Formations. II5 pp. 4 pls. Palaeontographical Society, London.

PARRINGTON, F. R. I935. A Note on the Parasphenoid of the Cynodont Thrinaxodon liorhinus Seeley. Ann. Mag. Nat. Hist., London (Io) $16: 399-40$ r, fig.

- I936. On the Tooth-Replacement in Theriodont Reptiles. Philos. Trans., London (в) 226 : I2I-I42.

-. I946. On the Cranial Anatomy of Cynodonts. Proc. Zool. Soc. London, 116 : I8I-I97, Io figs.

-. I947. On a Collection of Rhaetic Mammalian Teeth. Proc. Zool. Soc. London, 116 : 707-728, pl. I.

PARRINGTON, F. R. \& WESTOLL, T. S. I940. On the Evolution of the Mammalian Palate. Philos. Trans., London (B) $230: 305-355$, I6 figs.

PEARSON, H. S. I924. A Dicynodont Reptile Reconstructed. Proc. Zool. Soc. London, 1924 : 827-855, figs. I9-39.

- I926. Pelvic and Thigh Muscles of Ornithorhynchus. J. Anat., London, $60: \mathrm{I}_{52}^{2-163}, 6$ figs.

RICHARDSON, L. I903. Mesozoic Geography of the Mendip Archipelago. Proc. Cotteswold Nat. Fld. Cl., Gloucester, 14:59-73, I fig.

I905. The Rhaetic and Contiguous Deposits of Glamorganshire. Quart. J. Geol. Soc. London, 61:385-424, pl. 33 .

—. I9II. The Rhaetic and Contiguous Deposits of West, Mid, and part of East Somerset. Quart. J. Geol. Soc. London, 77 : I-74, pls. I-4.

ROMER, A. S. I922. The Locomotor Apparatus of Certain Primitive and Mammal-like Reptiles. Bull. Amer. Mus. Nat. Hist., New York, 46 : 5I7-606, pls. 27-46.

—. I924. The lesser Trochanter of the mammalian Femur. Anat. Rec., Philadelphia, 28 : 95-I02. I945. Vertebrate Palaeontology. 2nd ed., viii +687 pp., 377 figs. Chicago.

ROMER, A. S. \& PRICE, L. I. I940. Review of the Pelycosauria. Spec. Pap. Geol. Soc. Amer., Washington, $28: \mathrm{I}-538$, pls. I-46.

SCHAEFFER, B. I94I. The Morphological and Functional Evolution of the Tarsus in Amphibians and

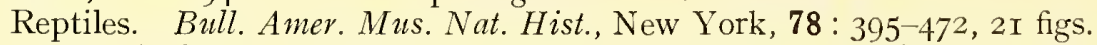

- I941a. The Pes of Bauria cynops Broom. Amer. Mus. Novit., New York, 1103: I-7, 4 figs.

SEELEY, H. G. I879. Note on a Femur and a Humerus of a small Mammal from the Stonesfield Slate. Quart. J. Geol. Soc. London, 35:456-463, 2 figs.

I895. Researches on the Structure, Organization, and Classification of the Fossil Reptilia, IX, 5. On the Skeleton in New Cynodontia from the Karroo Rocks. Philos. Trans., London (B) 186 : 59-I48, 34 figs.

SIMPSON, G. G. 1928. A Catalogue of the Mesozoic Mammalia in the Geological Department of the British Museum. x +2 I5 pp., I2 pls. British Museum (Nat. Hist.), London.

I928a. Mesozoic Mammalia, XII. The Internal Mandibular Groove of Jurassic Mammals. Amer. J.

Sci., New Haven (5) $15: 46 \mathrm{I}-47 \mathrm{O}, 7$ figs.
I933. The Ear Region and the Foramina of the Cynodont Skull. Amer. J. Sci., New Haven (5) 26 : 285-294, 5 figs.

—. I937. Skull Structure of the Multituberculata. Bull. Amer. Mus. Nat. Hist., New York, 73: 727-763, 9 figs.

- I938. Review of a discovery of Triassic Animals. Amer. J. Sci., New Haven (5) 35 : I44-I46.

1945. The Principles of Classification and a Classification of the Mammals. Bull. Amer. Mus. Nat. Hist., New York, $85:$ xvi $+35^{\circ} \mathrm{pp}$.

- - I947. Haramiya, new name, replacing Microcleptes Simpson, I928. J. Paleont., Tulsa, $21: 497$.

SLIJPER, E. J. I946. Comparative Biologic-Anatomical Investigations on the Vertebral Column and Spinal Musculature of Mammals. Verh. Akad. Wet. Amst. (2) 42 : I-I28.

Trueman, A. E. I922. The Liassic Rocks of Glamorgan. Proc. Geol. Ass. London, $33: 245^{-284}$, pls. 8-Io.

TUTCHER, J. W. \& TRUEMAN, A. E. I925. The Liassic Rocks of the Radstock District. Quart J. Geol. Soc. London, $81: 595-666$, pls. 38-4I. 
VIRCHOW, H. I9I3. Wirbelsäule und Rotatoren der Bären. Arch. Anat. Physiol., Leipzig, 1913:41-92, 6 figs.

WATSON, D. M. S. IgII. The Skull of Diademodon, with Notes on those of some other Cynodonts. Ann. Mag. Nat. Hist., London (8) $8: 293-330,9$ figs.

-. IgI2. On some Reptilian Lower Jaws. Ann. Mag. Nat. Hist., London (8) $10: 573-587$.

- I9I3. Further Notes on the Skull, Brain, and Organs of Special Sense of Diademodon. Ann. Mag. Nat. Hist., London (8) $12: 2 \mathrm{I} 7-228,5$ figs.

- I916. The Monotreme Skull: A Contribution to Mammalian Morphogenesis. Philos. Trans., London (B) $207: 3$ II -374 , pls. $23-25$.

—. I9I7. The Evolution of the Tetrapod Shoulder Girdle and Forelimb. J. Anat., London, 52 : I-63, 27 figs.

I920. On the Cynodontia. Ann. Mag. Nat. Hist., London (9) $6: 506-524$, I3 figs.

- I942. On Permian and Triassic Tetrapods. Geol. Mag., London, 79 : 8I-II6, 5 figs.

WESTLING, C. I889. Anatomische Untersuchungen über Echidna. Bih. svensk. VetenskAkad. Handl., Stockholm, 15, 4, 3: I-7I, pls. I- 6 .

WILSON, J. T. \& McKAY, W. J. S. I894. On the Homologies of the Borders and Surfaces of the Scapula in Monotremes. Proc. Linn. Soc. N.S.W., Sydney (2) $8: 377-388$, pl. 21 .

WOODIVARD, A. S. I898. Outlines of Vertebrate Palaeontology for Students of Zoology. xxiv + 470 pp., 228 figs. Cambridge.

- I925. In Zittel, K. A. von. Text-Book of Palaeontology, 3. Mammalia. viii +316 pp. 374 figs. (Translation revised, with additions.)

YOUNG, C. C. I940. Preliminary Notes on the Mesozoic Mammals of Lufeng, Yunnan, China. Bull. Geol. Soc. China, Peking, 20 : 93-III, II figs.

- I947. Mammal-like Reptiles from Lufeng, Yunnan, China. Proc. Zool. Soc. London, $117: 537-597$. pls. I-4. 



\section{INDEX}

Abel, O., I4

Acrodus anningiae, 7

minimus, 7

Aelurognathus, I3o

American Museum of Natural History, 74

Ammonoidea, 7

Ancestors (of Tritylodontidae), I37

Arnioceras, 7

Articular, 36, 38 (fig.)

Artiodactyls, 63

Astragalus, I30 (fig.), I3I

Ateles, Io9

Bairstow, L., 6, 7

Barnicot, N. A., 4

Basioccipital, 48

Basisphenoid, 52

Bate, Miss D. M. A., 4

Bauriamorphs, I37-I38

Belemnoidea, 7

Bienotherium, 24, 34, 40, 42, 43 (fig.), 44, 45, 52, 56, $57,59,76,97$, II 8, I 26, I 27, I 36, I3 8, I 39

elegans, 97

minor, 93, 97

yunnanense, 43 (fig.), 97, I36, I38, I39

Bohlin, B., 3, I39

Bones, fracture of, Io

Brachiopoda, 7-9

Brain, 53, 54 (fig.)

case, $46,5 \mathrm{I}$ (fig.)

Brodie, W. R., I

Broom, R., I38

Bruce, J. R., 4

Butler, P. M., 3, 7I

Calcaneum, I29 (fig.)

Canis, 29

Carpus, 129

Castor, 106

Cheek teeth, lower, 76

upper, 67

Clavicle, II4 (fig.)

Claw, I34 (fig.), I35

Cox, L. R., 6, 7

Ctenacodon, I39.

Cuthbert, C. R., 4

Cynodonts, 22, 23, 30, 3I, 34-38, 40, 42, 43, 46, 48, $52,54,55,59,62-65,67$, I00, I04, I05, II3, II5, II 8, I20, I22, I24, I37-I39, I42

Cynognathus, 28, 29, 36, 38, 39, I05, I30, I4I

Dentary, 3I-33 (fig.), 34, 35
Dentition, 67

function of, 93

Diademodon, 26-28, 35, 36, 40, 4I (fig.), 42, 43, 45, $46,49,50,52,53,56-58,63,98,99$ (fig.), I00, II5, II7, II8, I20, I22, I24-I26, I29, I30, I40, I4I

polyphagus, 39

Dicynodonts, 63

Didelphis, I08, Io9, I24, I28, I3I

Docodon, 35

Dromatherium, I36

Ear, inner, 52

middle, 63

Ecology of Oligokyphus, I7, I8

Edwards, W. N., v, 4.

Eoraetia siegerti, 2, I20, I22

Epipterygoid, 55 (fig.)

Ericiolacerta, 129

Erinaceus, 94, I25

Eucosmodon, I3I

Exoccipital, 46

Femur, I25, I26 (fig.)

Fibula, I28 (fig.)

Fracture incidence, Io, I4 (fig.), I5 (fig.)

Gastropoda, 7

Glamorgan, south, I4I

Gomphodontia, I37, I38

Gomphognathus cf. mastacus, 2I, 65 (fig.), II8

Gorgonopsia, 24, 29, II3, I38, I42

Gregory, W. K., I39

Hallau, I4I

Haramiya, I

Hennig, O., 76, 96, 97

Hipparion, I4

Hippotamus, 94

Hipposaurus, I29, I30

Holwell, I, I4I

Homo, I23, I25

Humerus, II7, II9 (fig.)

Hybodus minor, 7

Ictidosauria, 3, I36, I38, I42

Ictidosaurs A \& B, 33, 34, 63, I36, I39, I40

Ilium, I24

Incisors, 84

Interclavicle, II4

Ischium, I24

Jugal, 29, 30 (fig.) 
Karroomys, 136

Kunminia, 63, I36

Lacrimal, 26, 28 (fig.)

Lamellibranchiata, 7

Lemurs, I09

Lycaenops, I29, I30

Lycorhinus, I36

Mammals, origin of, I4O, I4I

skeletal characters, I4I

Manatus, 63

Marsupial bone, I24

Maxillary, 22, 25 (fig.)

Mendip I4 (see Windsor Hill Quarry)

Metacarpals, I32 (fig.)

Metapodials, I3I-I33

Metatarsals, I33 (fig.)

Microleptes, I

Microconodon, I36, I40

Microgomphodon, II 8, I29

Microlestes, I, I37

Microlestidae, I36, I37, I40

Moeritherium, 94

Monotremes, 52, 63, I04, I05, II3, II5, II7, I22, I24I26, I36, I39-I42

Moore, C., I

Morganucodon, I40

Mucrotherium, I39

Muir-Wood, H. M., 6-9

Multituberculates, 36, I38, I39

Nototherium, 63

Nythosaurus, 24, 52

larvatus, 27

Oligokyphus biserialis, 9, 76, 95, 96, 97

major, 6, 67, 75 (fig.), 83 (fig.), 98, 99 (fig.), I00, I05 minor, 6, 67, 75 (fig.), 83 (fig.), 98, I00 (fig.), I36

triserialis, 9, 93, 95-97

Ornithorhynchus, 50, 66, I00, I06, II3, II4, II7, I24, I27

Owen, R., I

Oxytoma inaequivalve, 7

Pachygenelus, I36

Palatine, 56

Pantotheria, 36

Parrington, F. R., 3, I36-I38

Parsons, L. E., 4

Pelvis, I23 (fig.)

Pelycosauria, I4I

Periotic, 48

Peyer, B., 3

Phalanges, I34 (fig.)

Pikermi, I4

Plagiaulacidae, I39

Plagiaulax, I39

Potamogale, I06

Premaxillary, I9, 2I (fig.)

Presternum, II3 (fig.)

Protacmon, 39, 55

Protodonta, I36, I39

Psendohastites, 7
Pterygoid, 56

Ptilodontidae, 139

Ptilodus, 46, 138, 139

Pubis, I24

Quadrate, 36, 39 (fig.)

Radius, I22 (fig.)

Reconstruction of skeleton, I37 (fig.) of skull, 59 (fig.)

Ribs, I I2 (fig.)

Romer, A. S., I3 8

Saurichthys acuminatus, 7

Sauropods, 54

Scapulo-coracoid, II5, II6 (fig.)

Scymnognathus, I29

Seeley, H. G., I38

Septomaxillary, 22, 48

Simpson, G. G., I, 36, 76, I20, I39

Skeleton, reconstruction of, I37 (fig.)

Skull, I9 reconstruction of, 59, 60-62 (figs.)

Spath, L. F., 6, 7

Sphenodon, 3, IIO

Splenial, 35

Squamosal, 40, 4I (fig.)

Stapes, 52

Stereognathus, II8, I26, I27

Sternum, II3 (fig.), II4

Stonesfield humerus (see Stereognathus)

Supraoccipital, $4^{8}$

Swanage, I

Symmetrodonts, 36

Systematics, 136

Tachyglossus, I0O, I05

Taeniolabis, I38, I39

Tarsus, I29

Taxonomy, 95

Teeth, incisors, 84, 85 (fig.), 88 (fig.), 90 (fig.), 92 (fig.) lower cheek, 76,77 (fig.), 83 (fig.)

succession of, 82

upper cheek, 67, 72 (fig.), 73 (fig.), 75 (fig.)

wear of, 82

Therapsids, Triassic, I29, I37

Theria, I40-I42

Theriodesmus, I4I

Thrinaxodon, 28, 39, 40, 44, 63

liorhinus, 2I, 64 (fig.)

Tibia, I27 (fig.)

Trechmann, C. T., 4

Triconodonts, 36

Triglyphus, 67

Tritheledon, 2, 3, I36, I38

Tritylodon, 22, 24, 26, 67, 93, 95, 96, II 8, г36, г38 longaevus, 58, 67, 7I, 93

Tritylodontidae, 3, 50, 63, 97, 136-142

Ulna, I20, I2I (fig.)

Uniserium, 139

Varanus, I35

Vertebrae, 98-III. 
Vertebral column, 98

Vertebrata, 6,7

Vomer, 56

Watson, D. M. S., 4, 98, Ir5, 126, 129, 138, 139

White, E. I., 6, 7
Wilcox, C., I

Windsor Hill Quarry, 4, 5, 10-13, 84, 85, 87, 92, 97 , 98 , II 4, I 24

Woodward, A. S., 138

Young, C. C., 76, 136

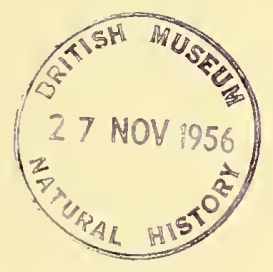



WITH THE EXCEPTION OF PLATE 4, FIG. I ALL THE PHOTOGRAPHS IN THE PLATES ARE STEREOSCOPIC.

PLATE I

OLIGOKYPHUS

II 
FIG. I.--Left maxillaries. $\times 3$.

(a) Medio-ventral view. R.7032.

(b) Medio-dorsal view of anterior end. R.7053.

(c) Medio-ventral view. R.7035.

FIG. 2.-Premaxillaries. $\times 3$.

(a) Right, ventral view. R.700I.

(b) Left, ventral view. R.7003.
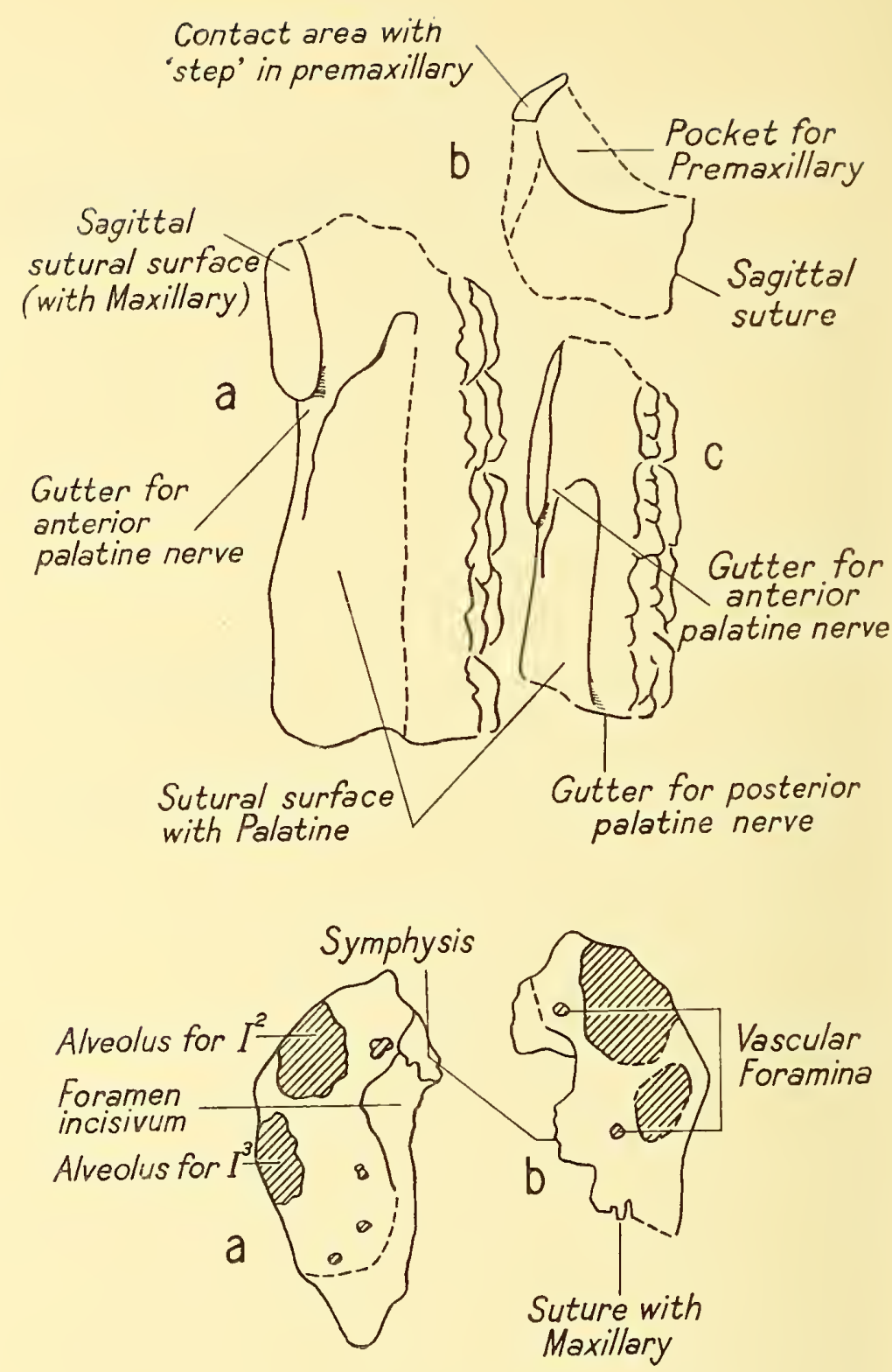

F1G. 3.-Premaxillaries. $\times 3$.

(a) Medial view, R.70or.

(b) Frontal view. R.7004.

(c) Lateral view. R.7002.
Contact area with
step' in premaxillary with Palatine 
Brit. Mus. (Nat. Hist.)

Plate 1

1
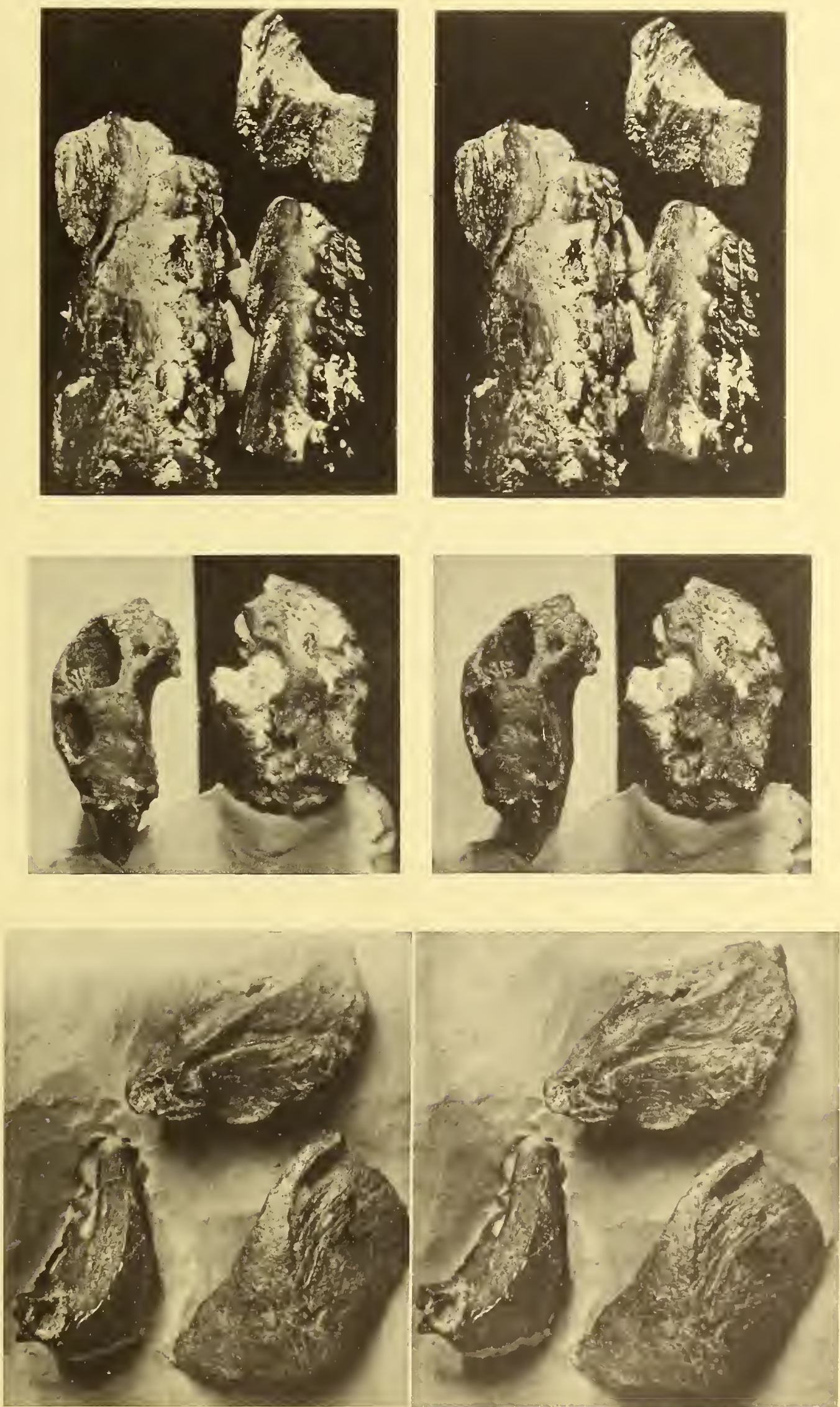

PLATE 2

OLIGOKYPHUS 
FIG. I.-Maxillaries in lateral view. $\times 3$.

(a) Left, anterior end. R.7053.

(b) Right, posterior end. R.7055.

Fig. 2.-Maxillaries in lateral view. $\times 3$.

(a) Right maxillary. The first cheek tooth is on the verge of being shed; its anterolateral root stands free without contact with bone. R.7028.

(b) Left maxillary. R.7027.

FIG. 3-Left maxillaries in ventral view. $\times 3$.

(a) R.7029.

(b) R.7027.

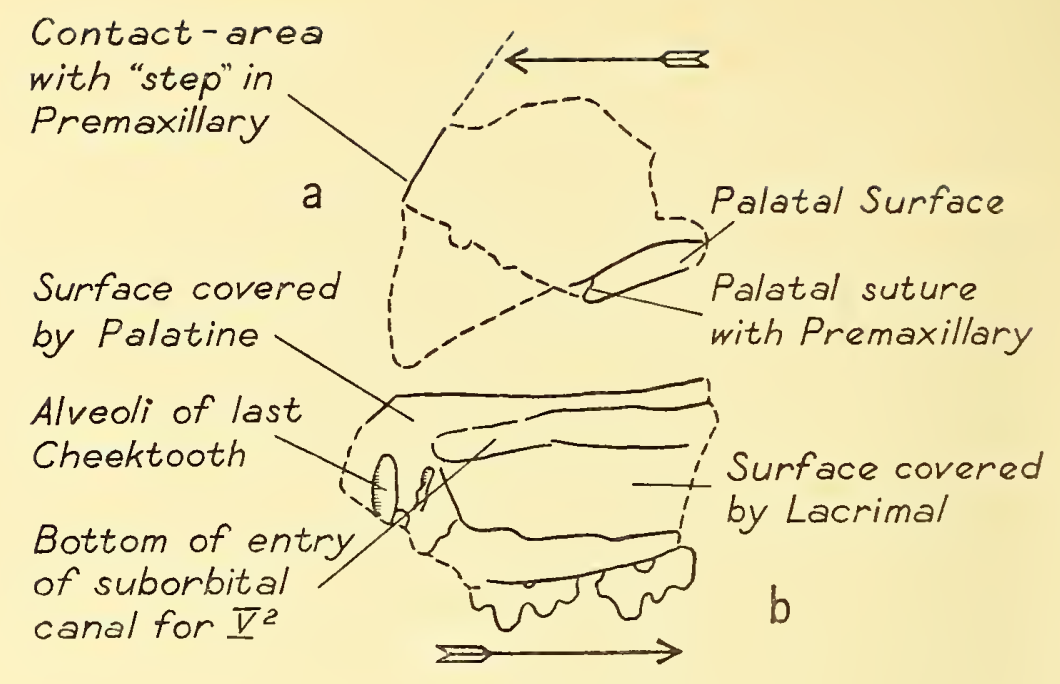

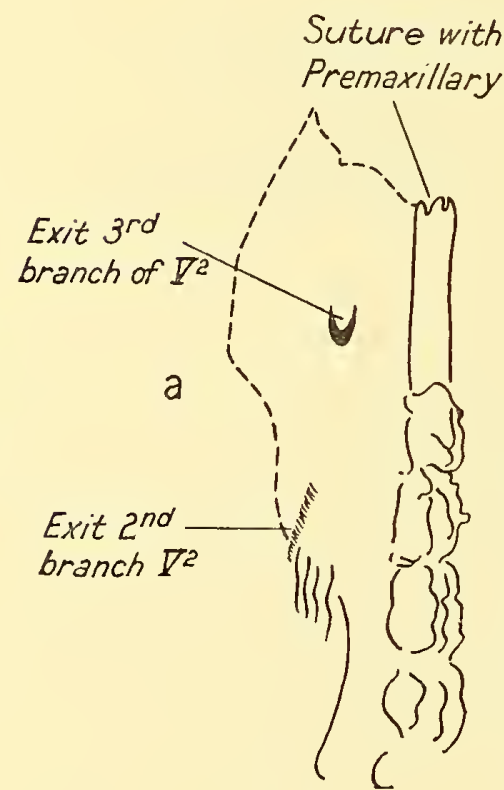

a

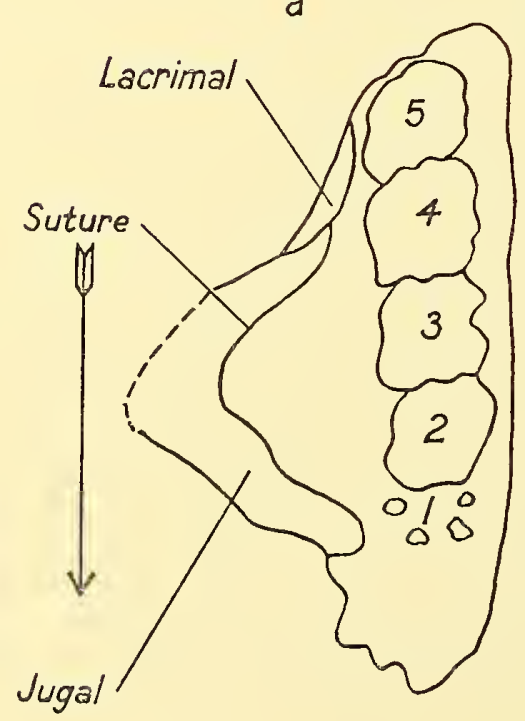

Suture with Palatine

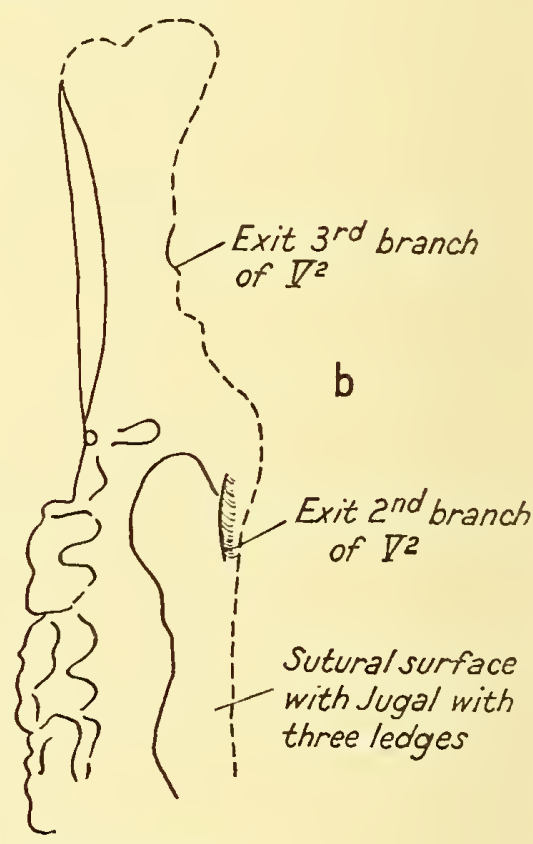

Suture with

Premaxillary b

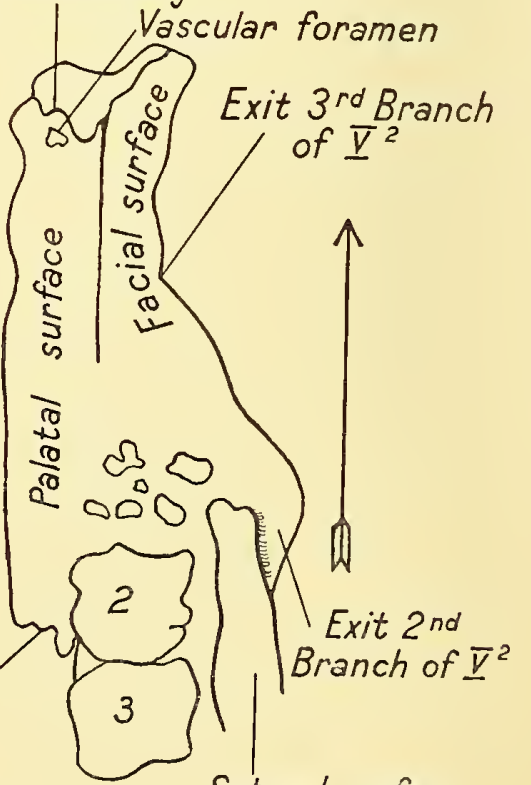

Sutural surface with Jugal 

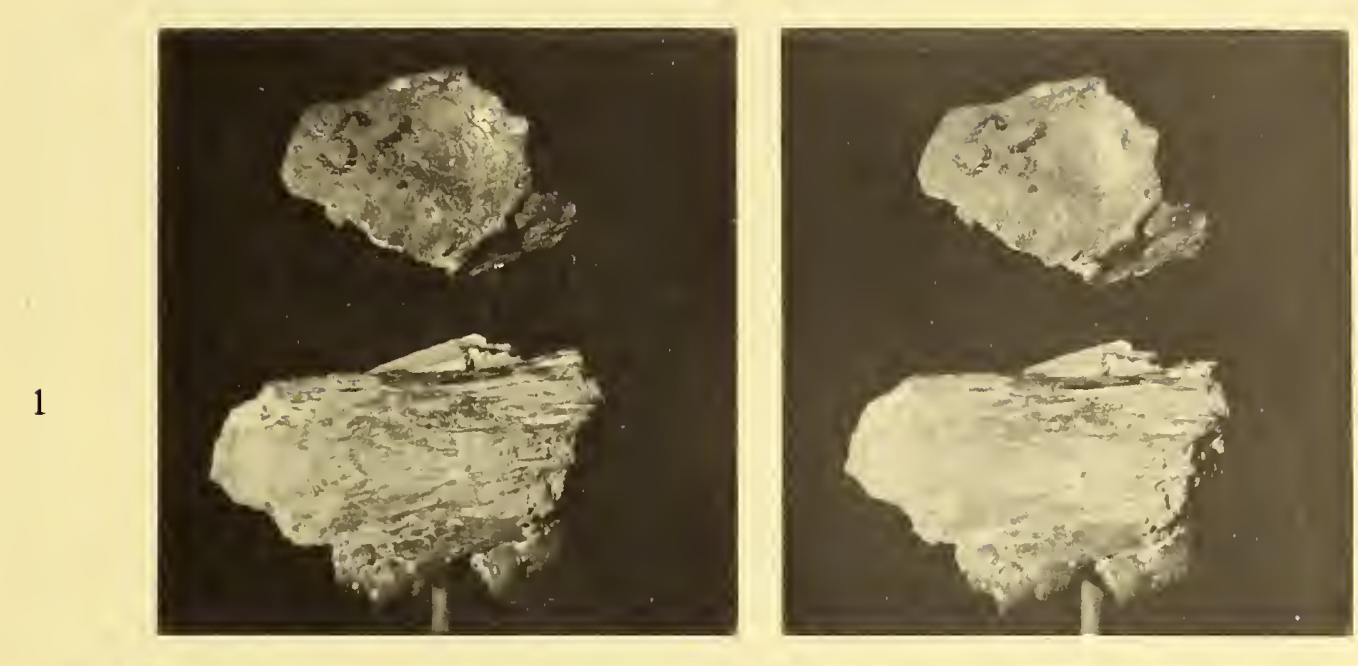

2
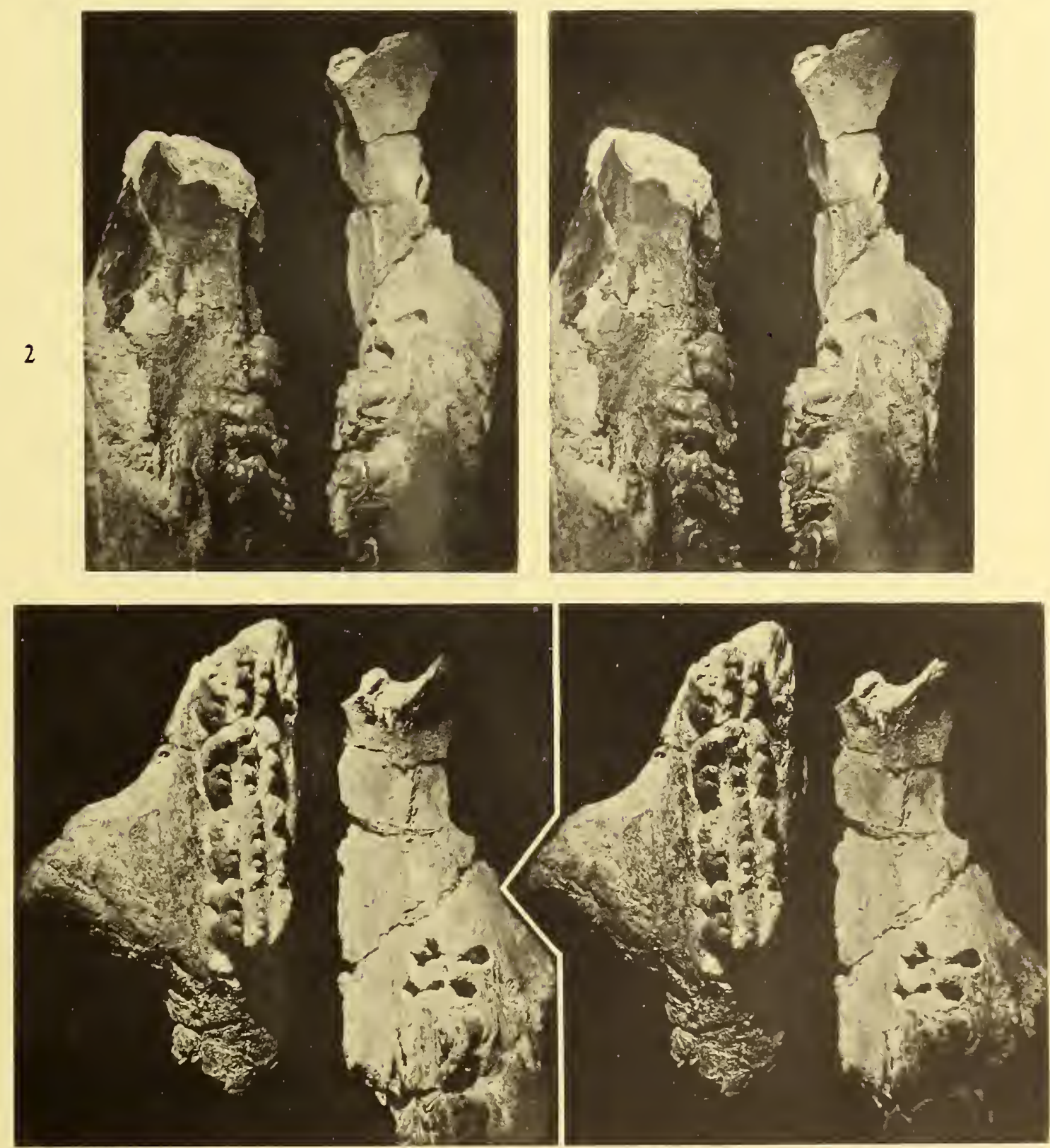

PLATE 3

OLIGOKYPHUS 
FIG. I.-Left jugals. $\times 2 \cdot 8$.
(a) view. R.7074.
(b) view. R.7070.
(c) Posterior view. R.7087. Associated with lacrimal.

FIG. 2.-Lacrimals. $\times 3$.

(a) Left, dorsal view. R.7059.

(b) Right, lateral view. R.7064

FIG. 3.-Left jugals. $\times 2 \cdot 6$.

(a) Dorsal view. R.7067.

(b) Lateral view. R.7069.

(c) Ventral view. R.7o7r.

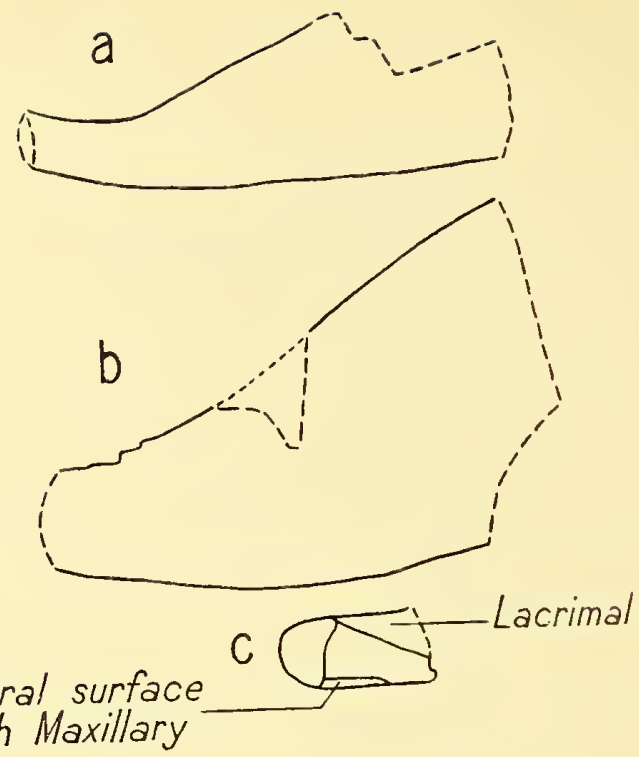

with Maxillary
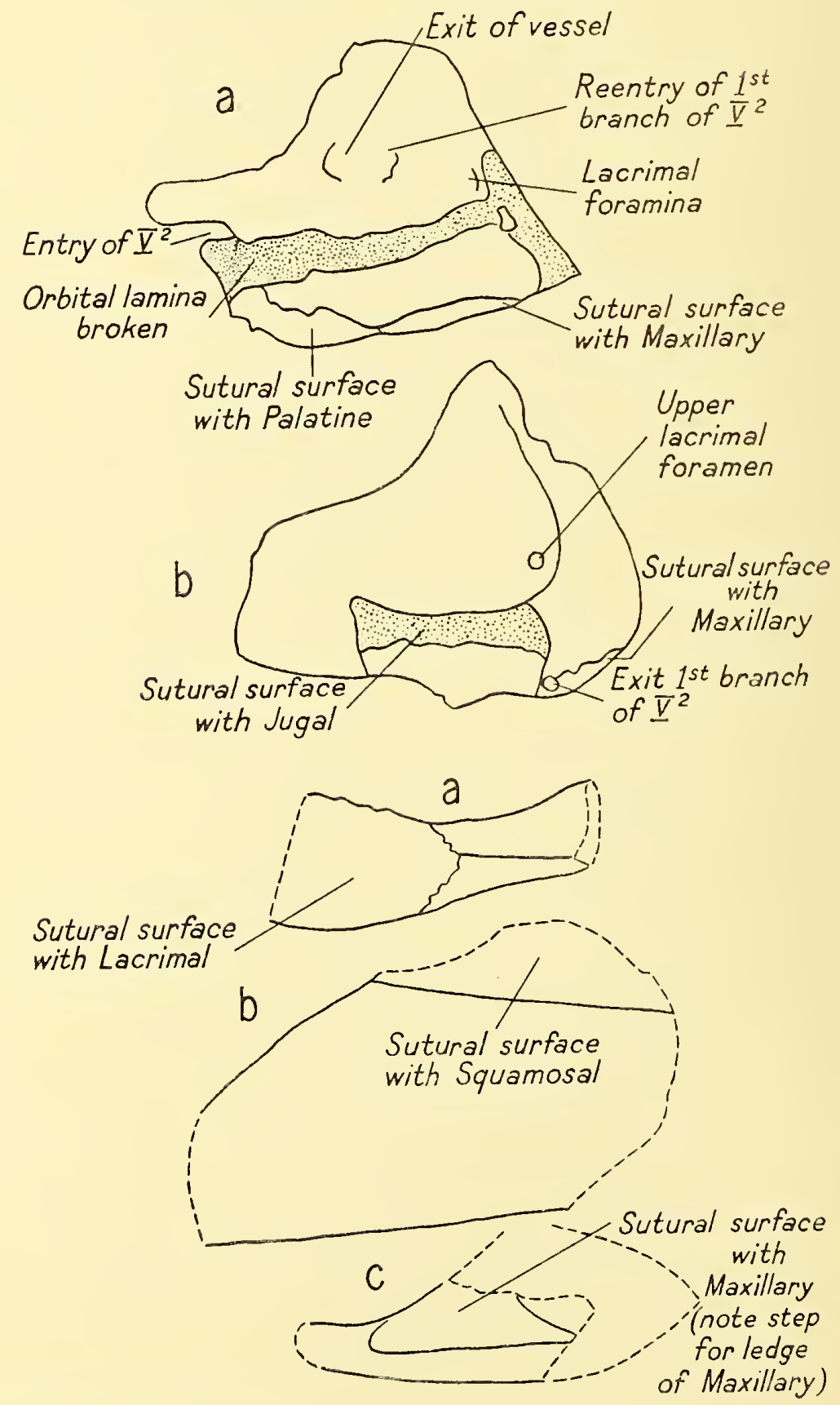

KEY TO PLATE 3 

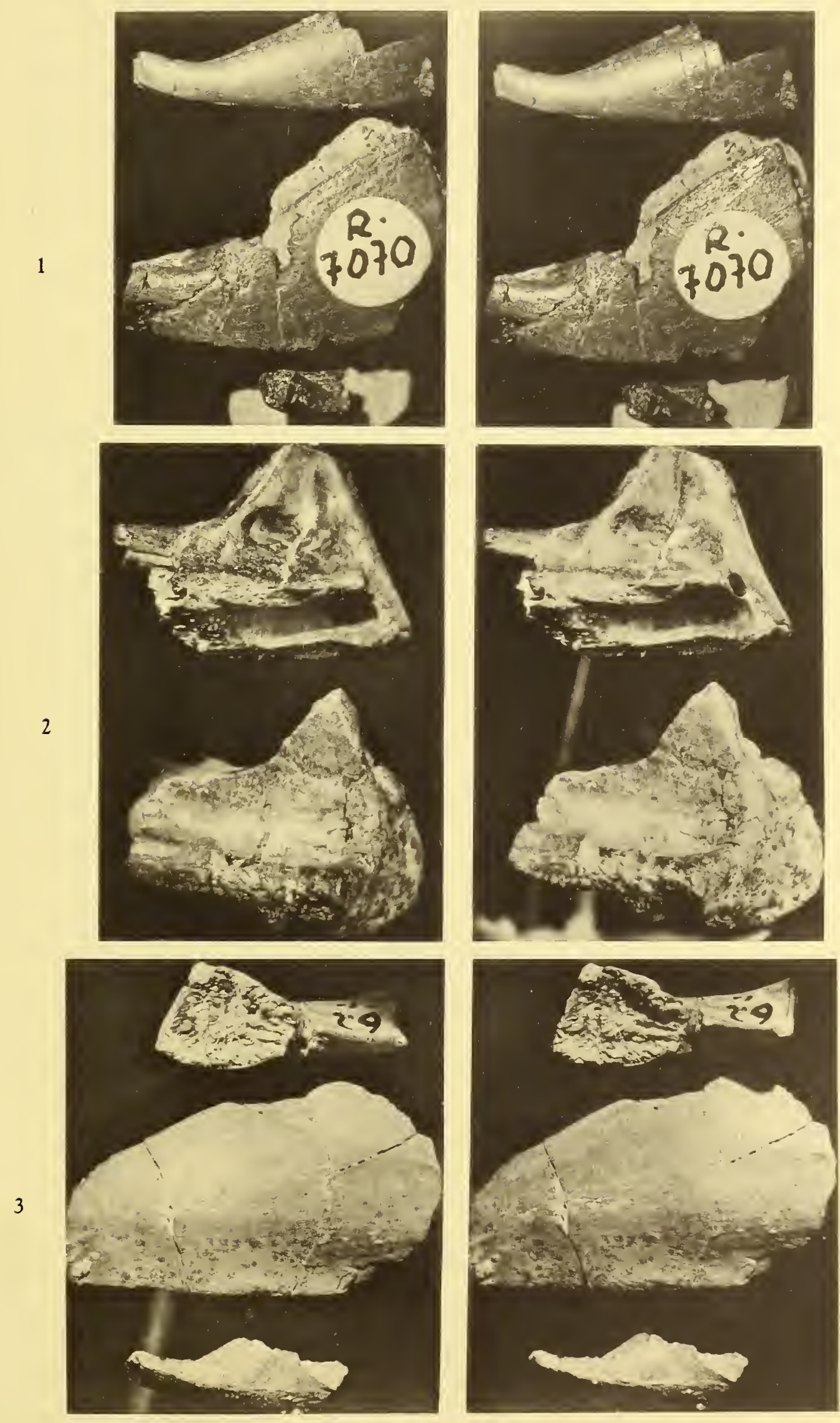

OLIGOKYPHUS 



\section{PLATE 4 \\ OLIGOKYPHUS}




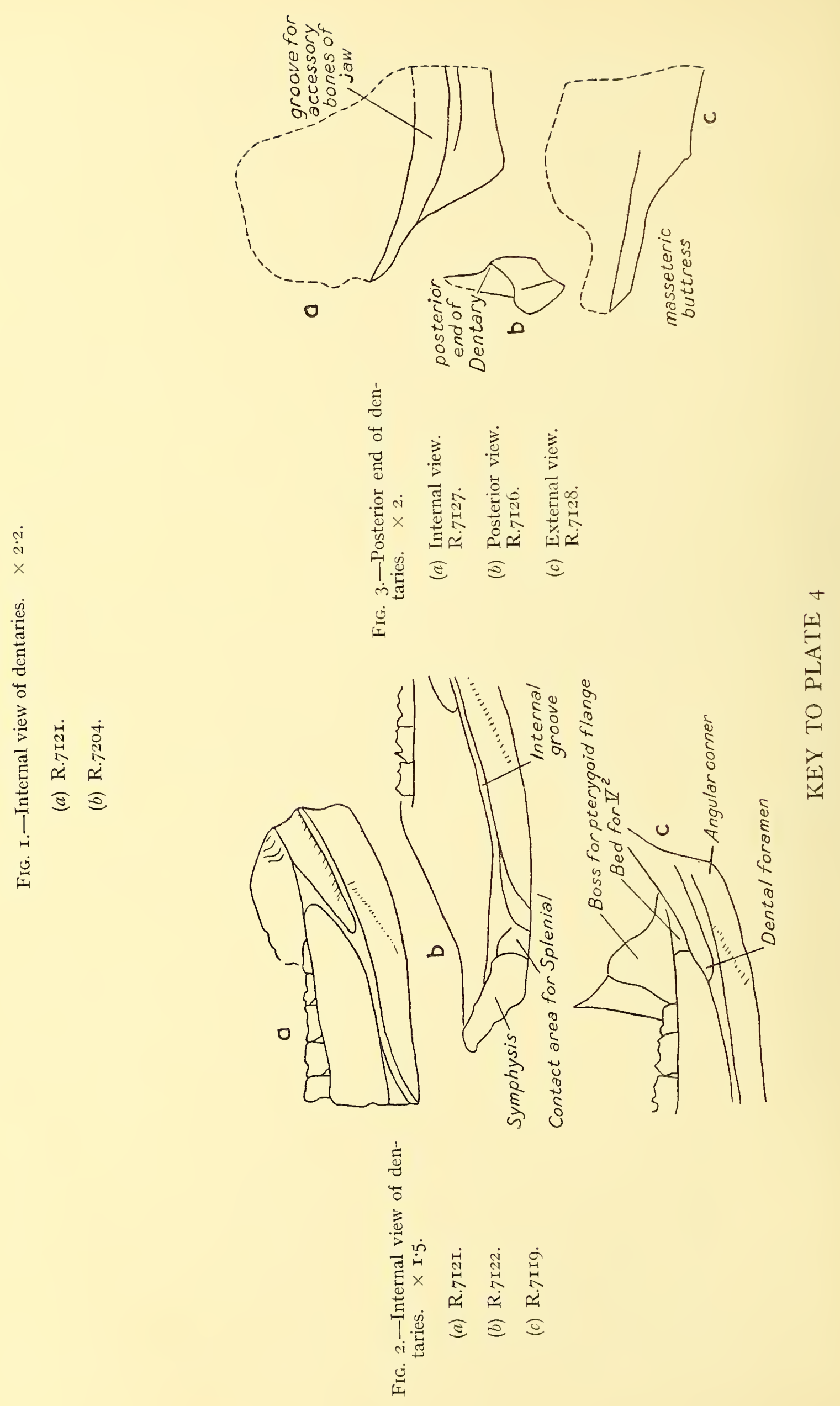


Brit. Mus. (NAt. Hist.)

PLATE 4
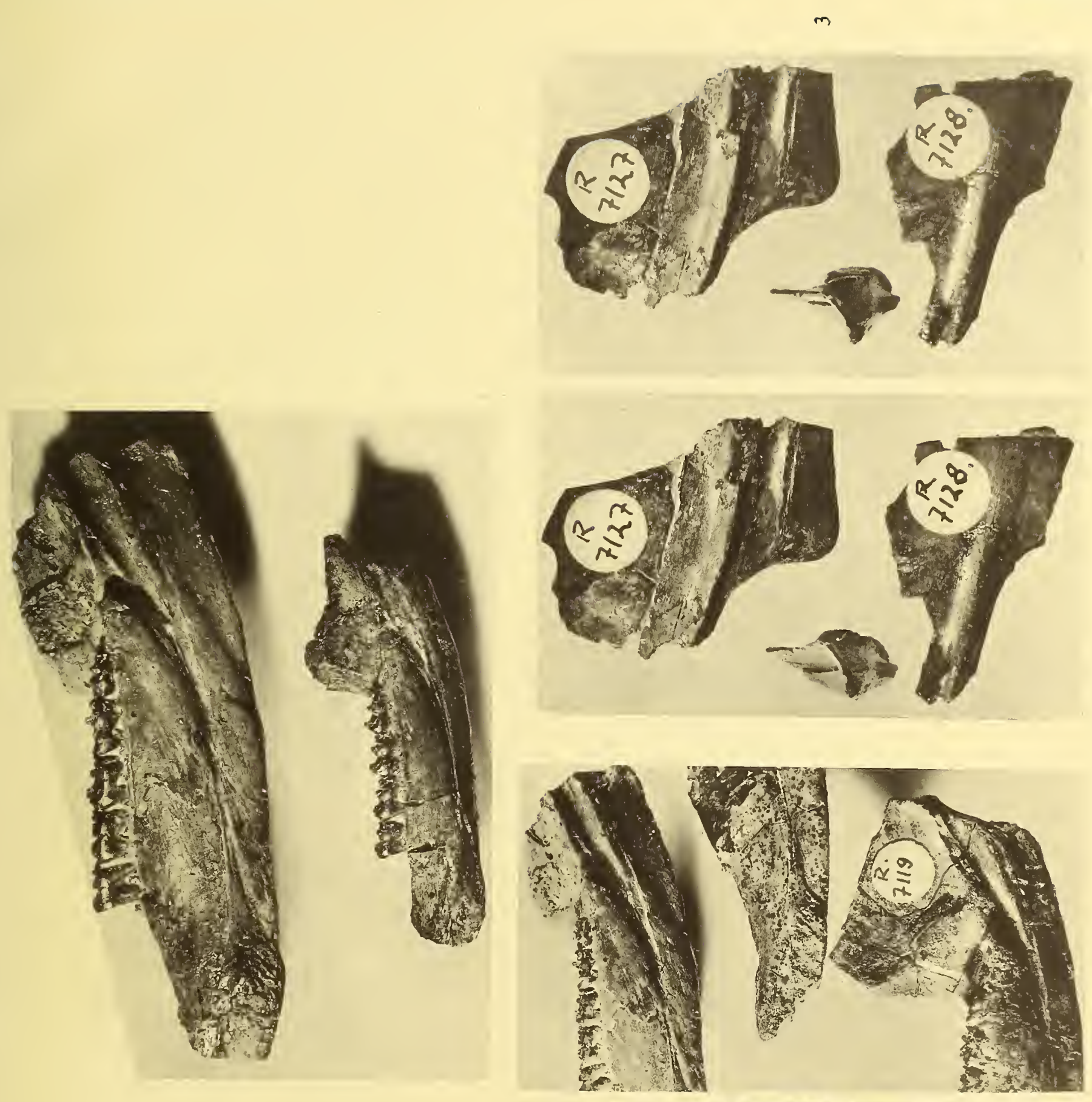

5
5
0
0
0
0

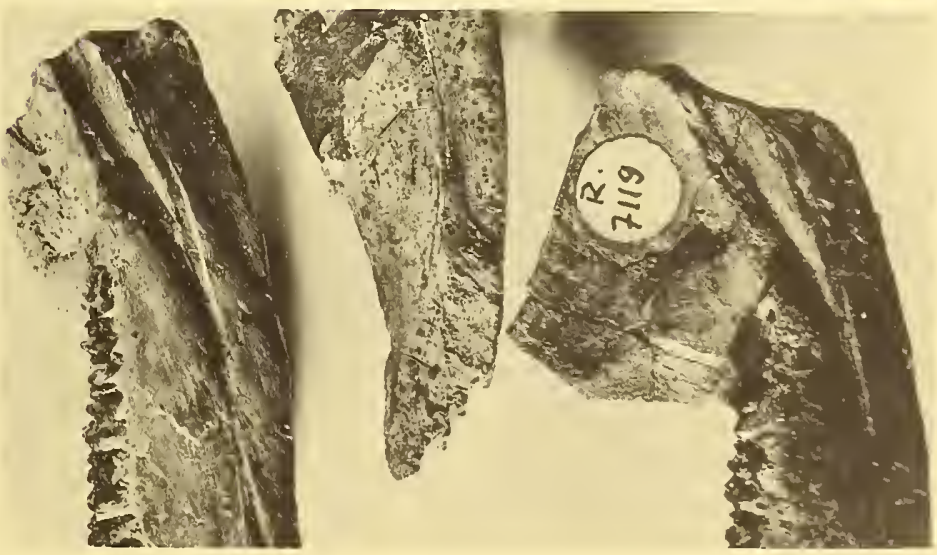





\section{PLATE 5 \\ OLIGOKYPHUS}



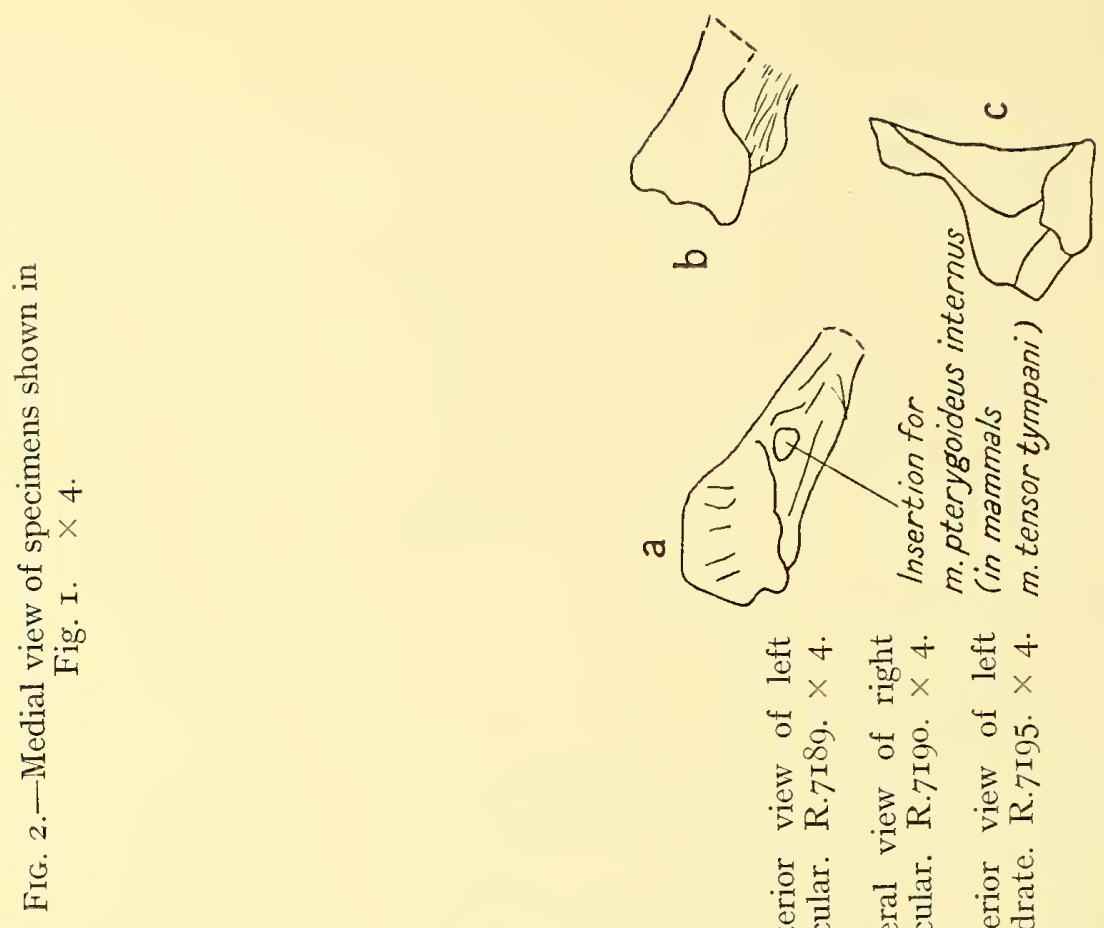

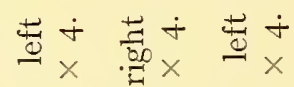

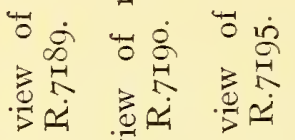

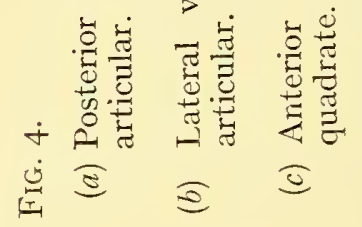
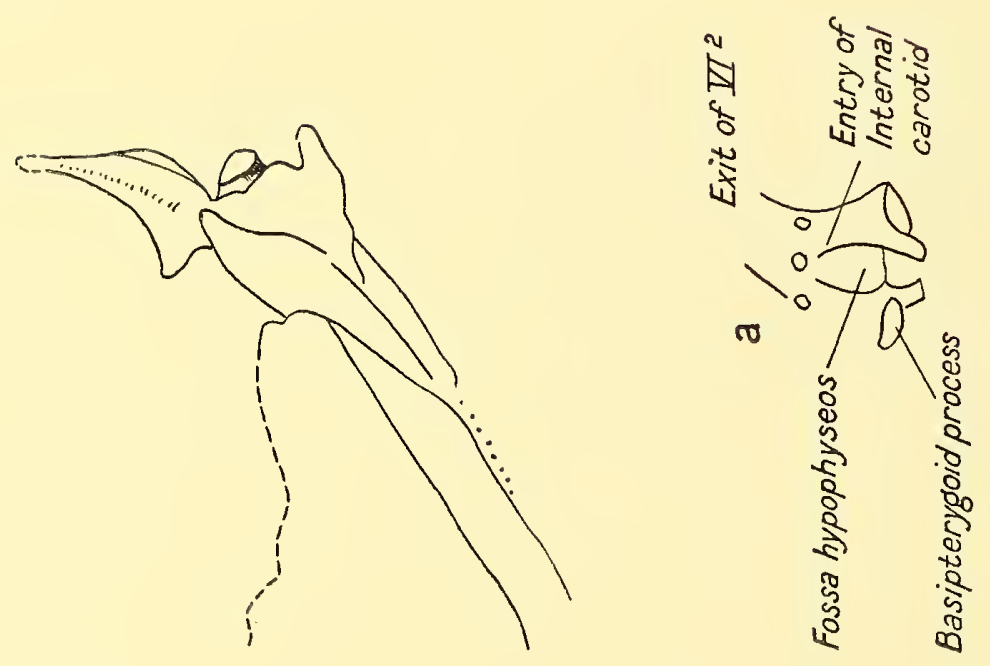

要
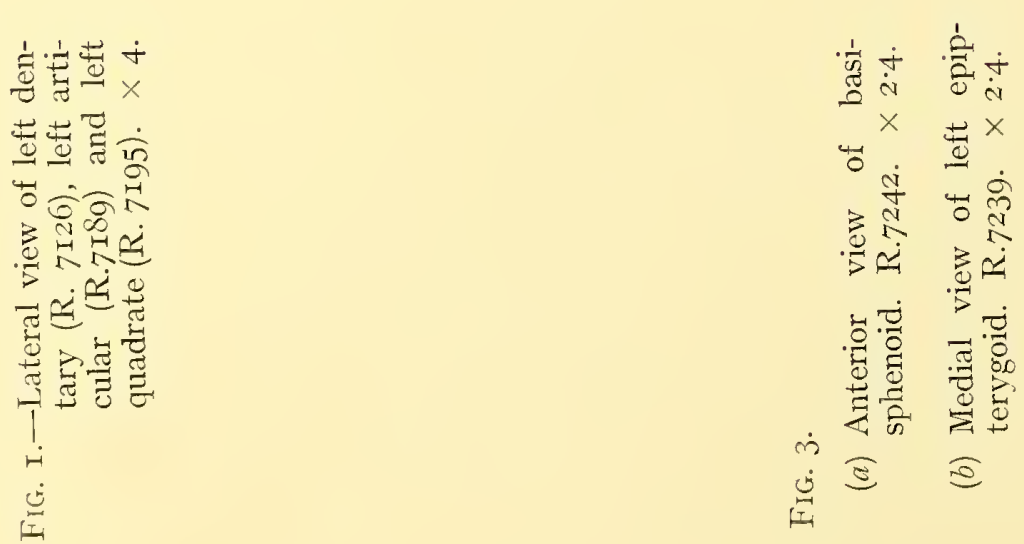
BRit. Mus. (NAT. Hist.)

Piate 5
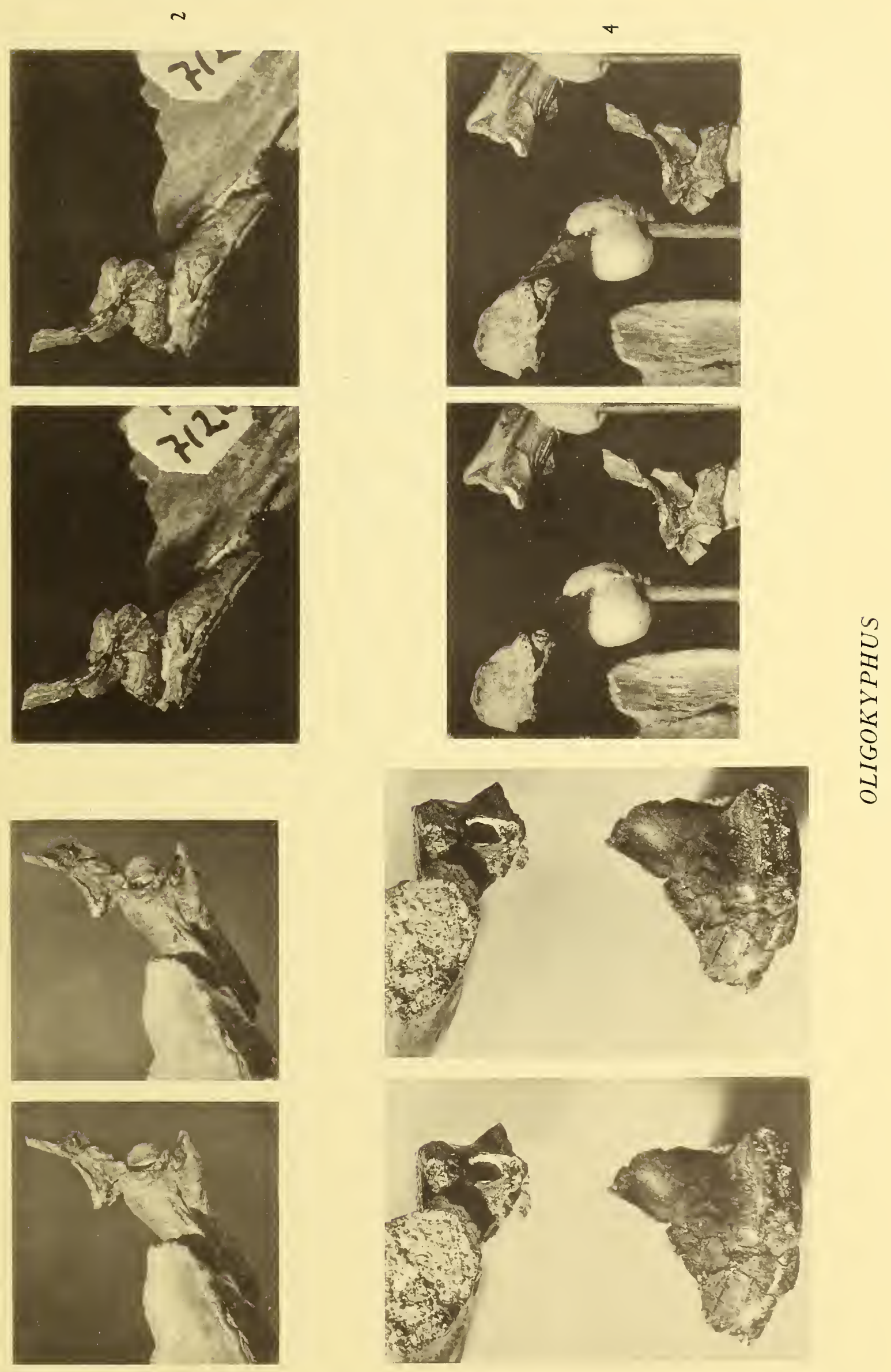

PLATE 6

OLIGOKYPHUS 


\section{a}

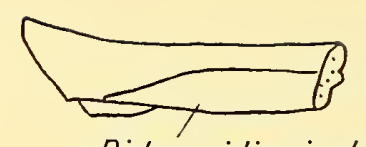

Ridge riding in duplication of Jugal

FIG. I.-Squamosals. $\times 3$.

(a) Left, ventral view. R.7Ior.

(b) Right, medial view. R.7097.

b

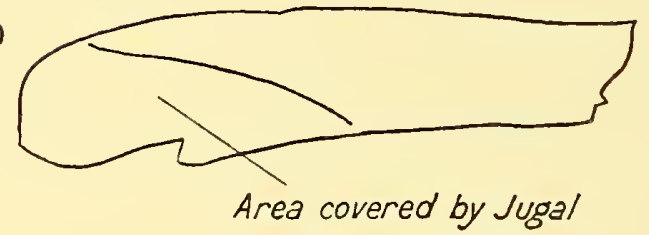

(c) Right, lateral view. R.7094.

(d) Right, lateral view. R.7098.

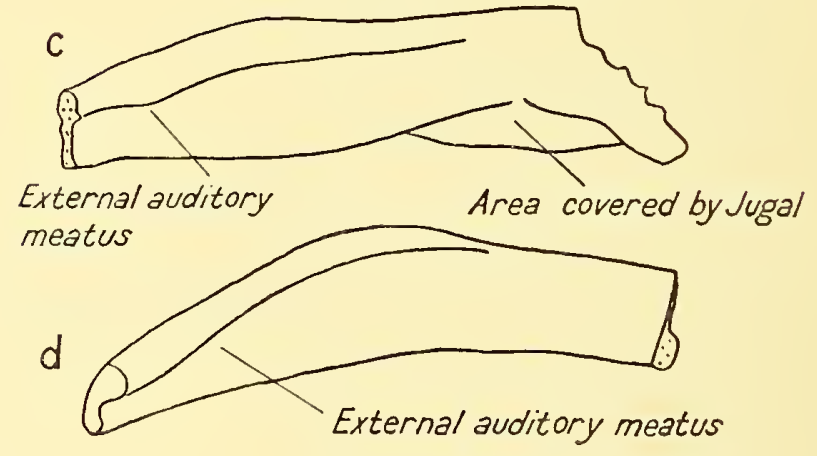

FIG. 2.-Right squamosals. $\times 2$.

(a) R.7205.

(b) R.7089.

(c) R.7088.

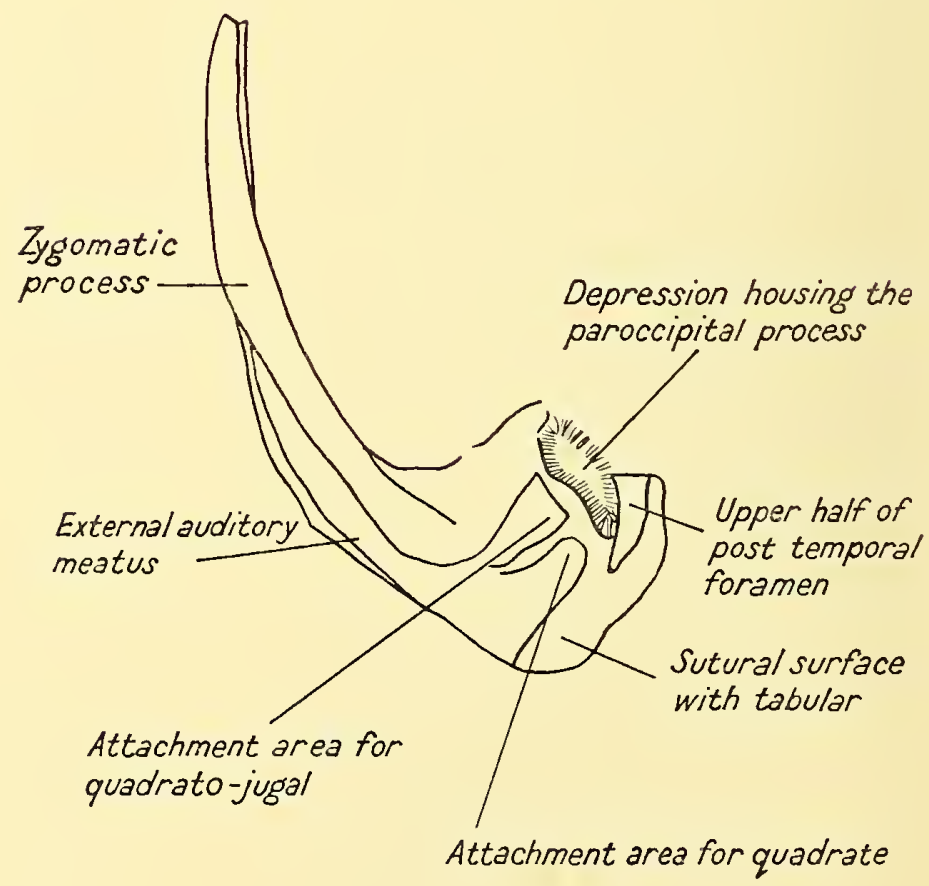

KEY TO PLATE 6 

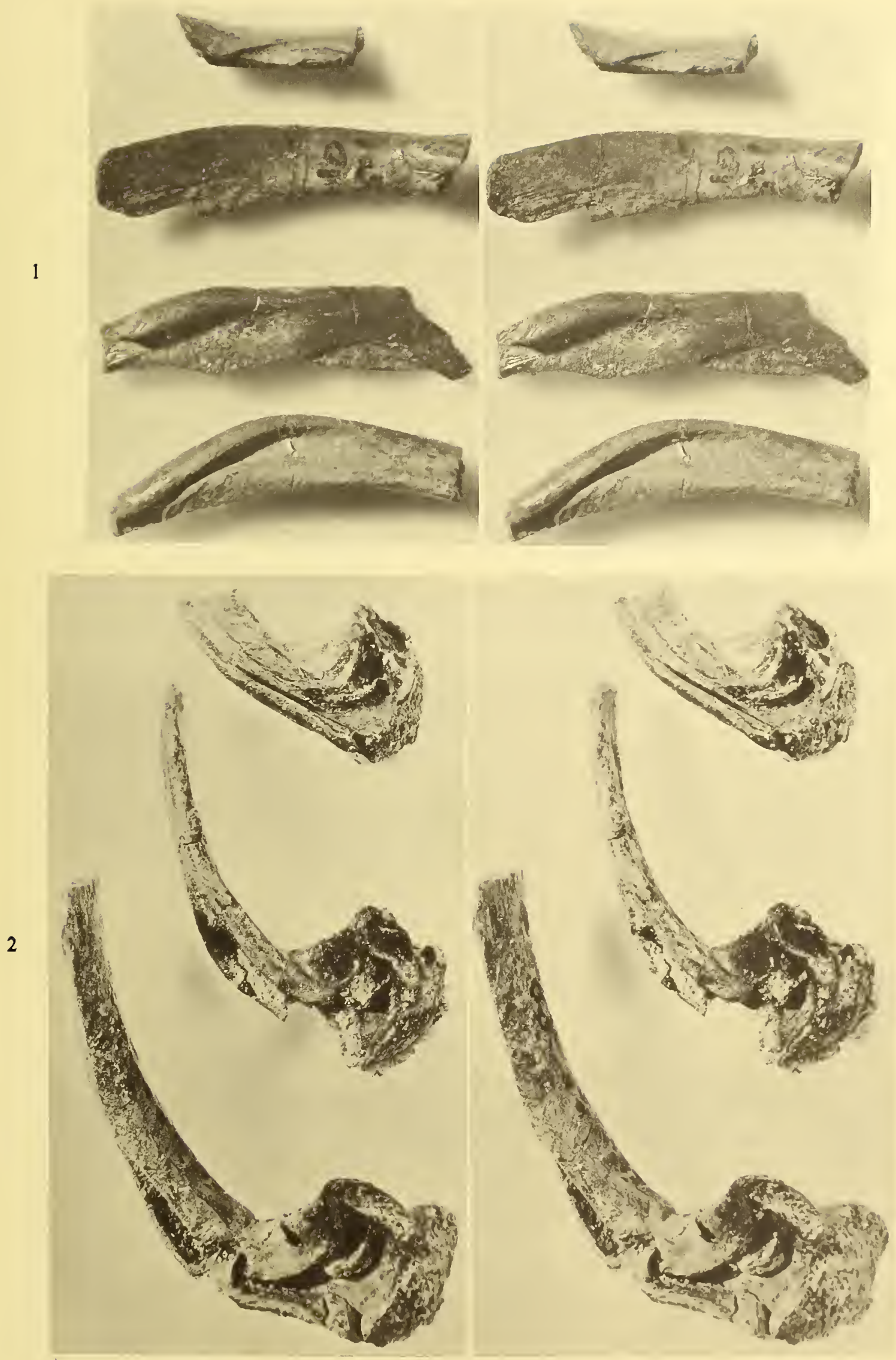



\section{PLATE 7 \\ OLIGOKYPHUS}



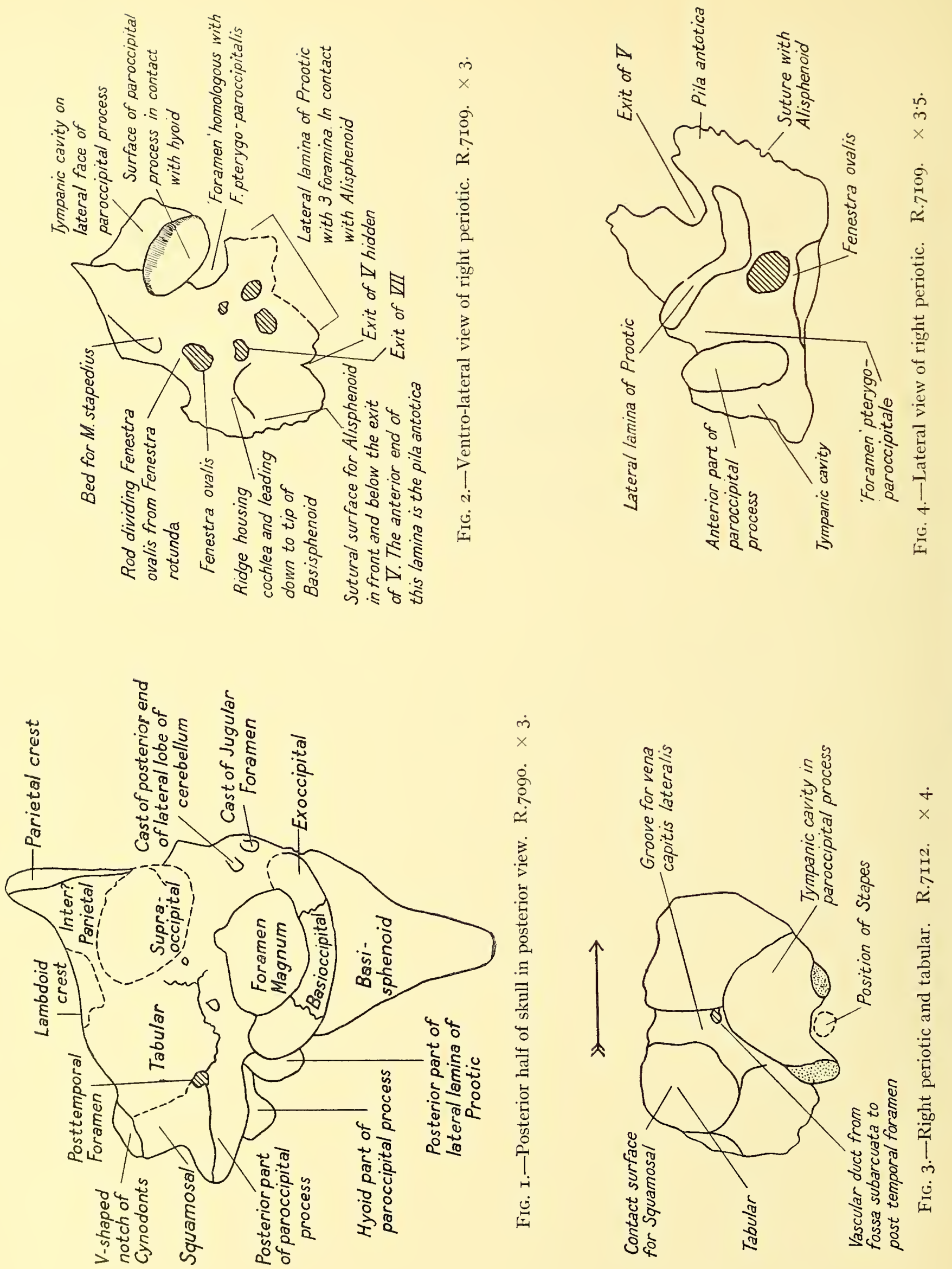

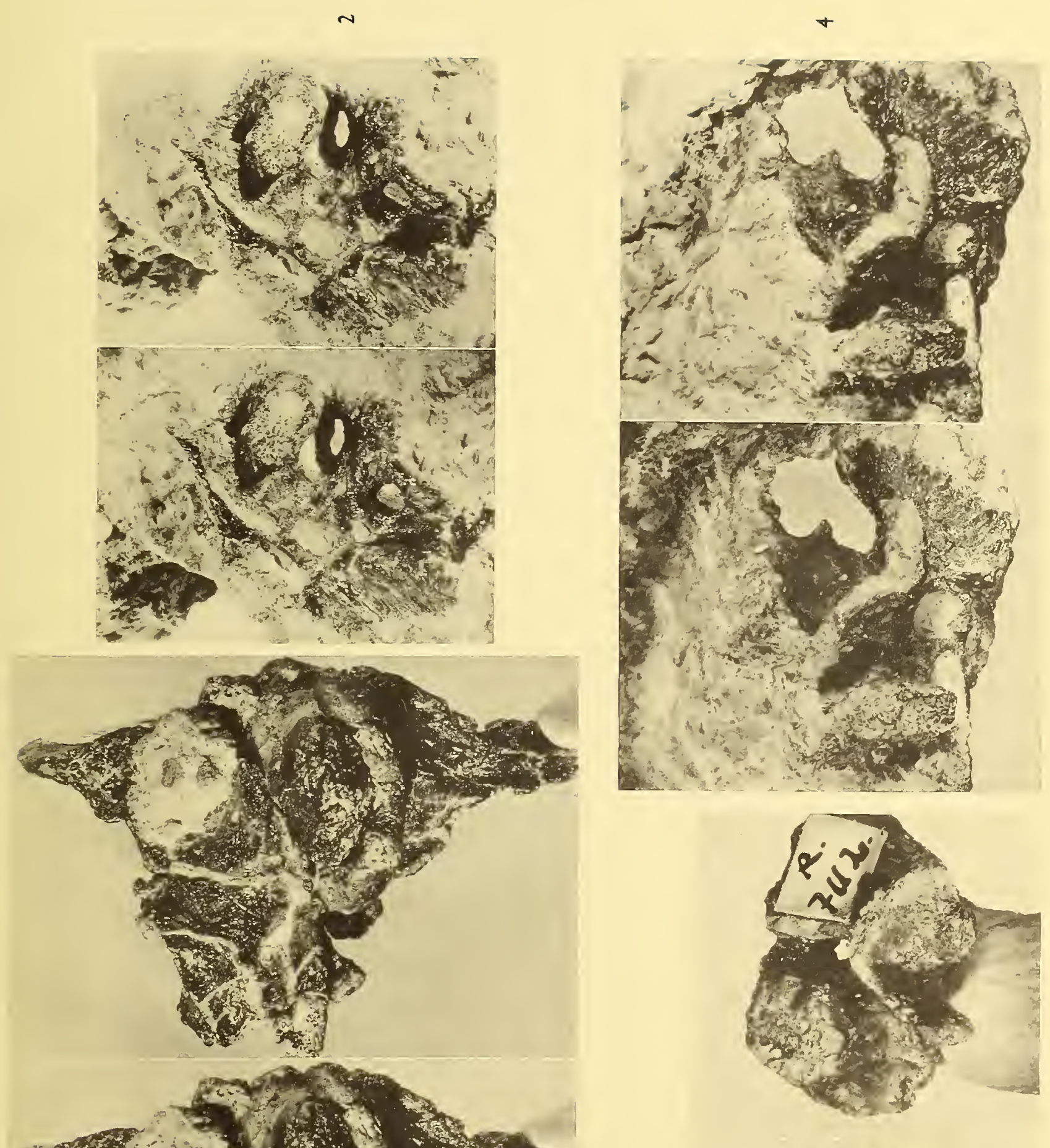

5
$\vdots$
$E$
0
0
0
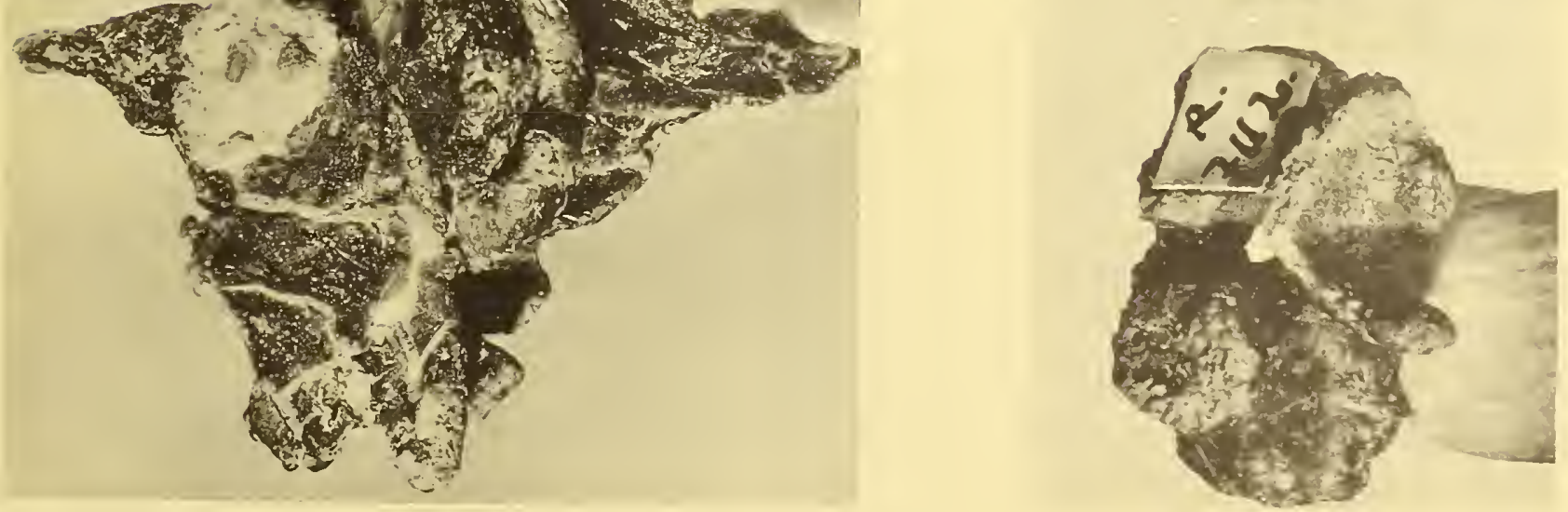



\section{PLATE 8 \\ OLIGOKYPHUS}


FIG. I.-Dorsal view of posterior half of skull. R.7090. $\times 3$.

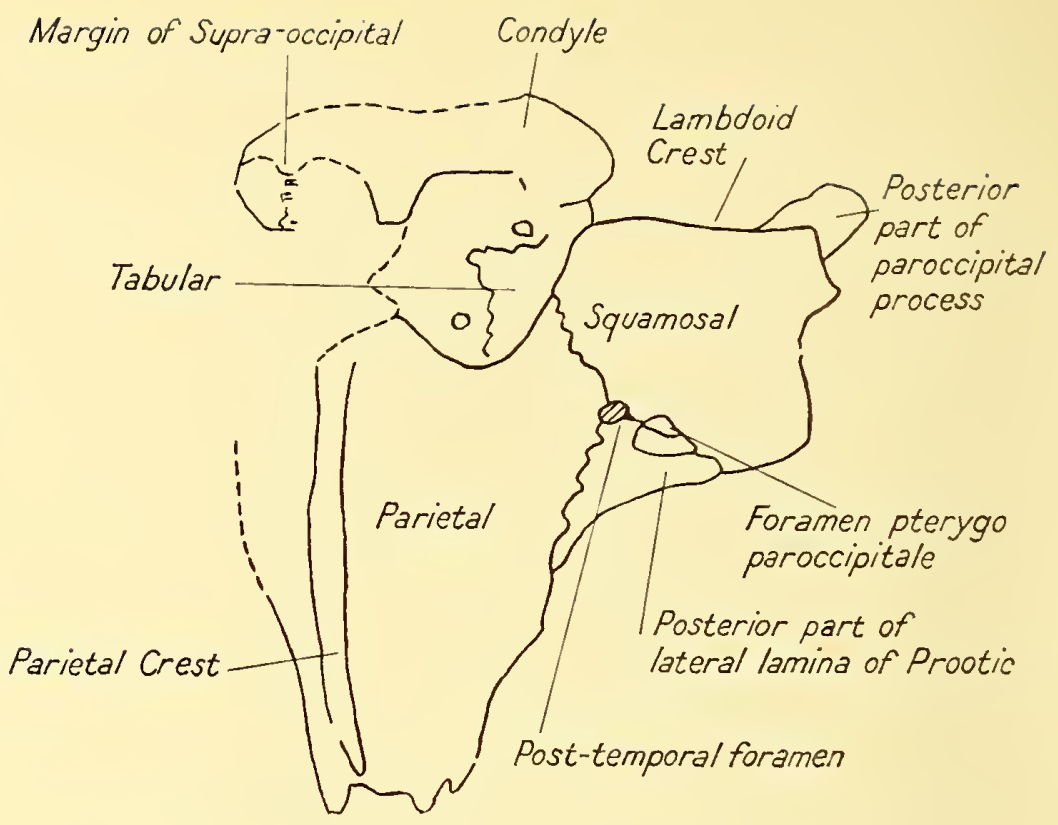

FIG. 2.-Ventral view of posterior half of skull. R.709o. $\times 3$.
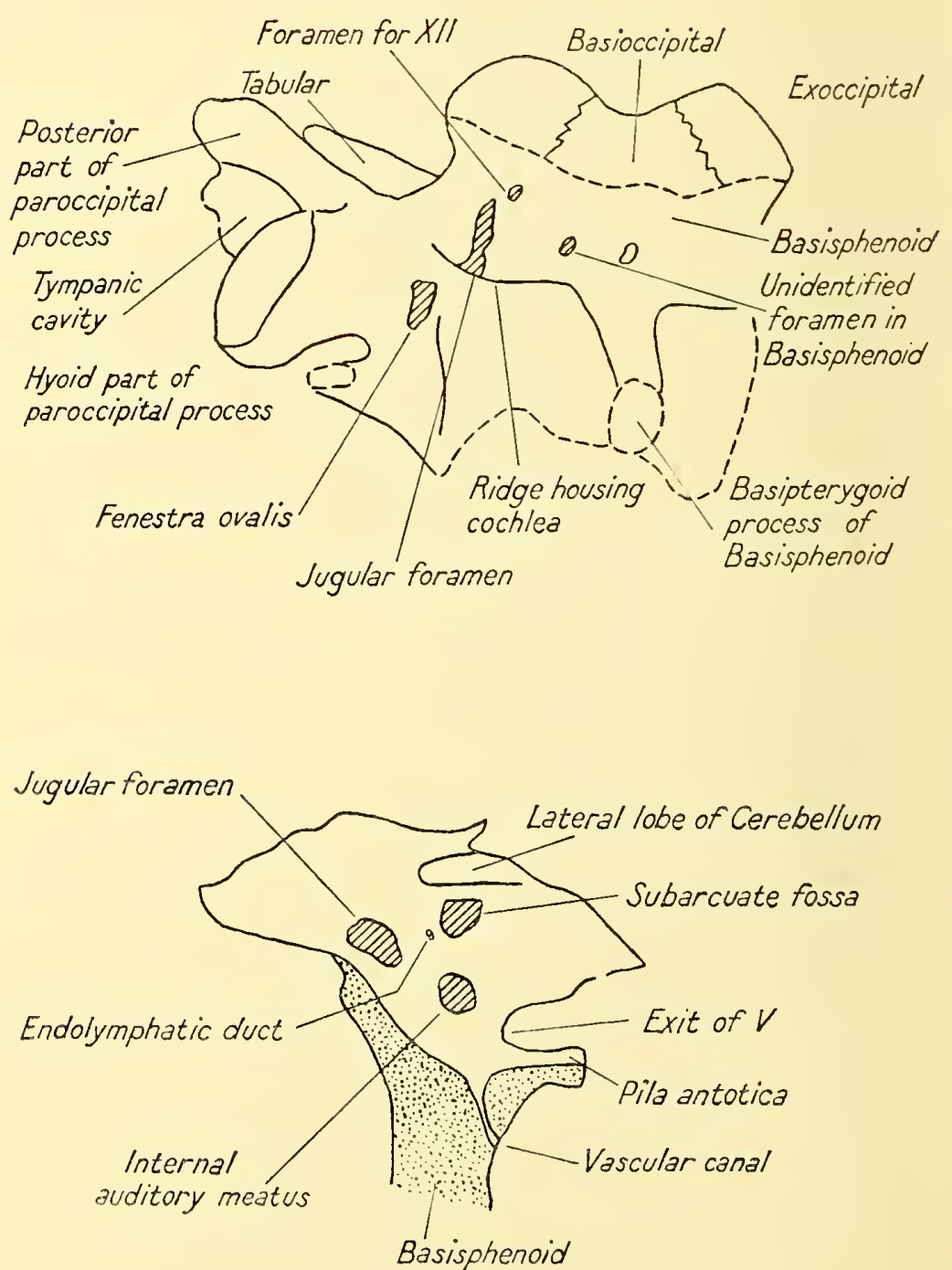

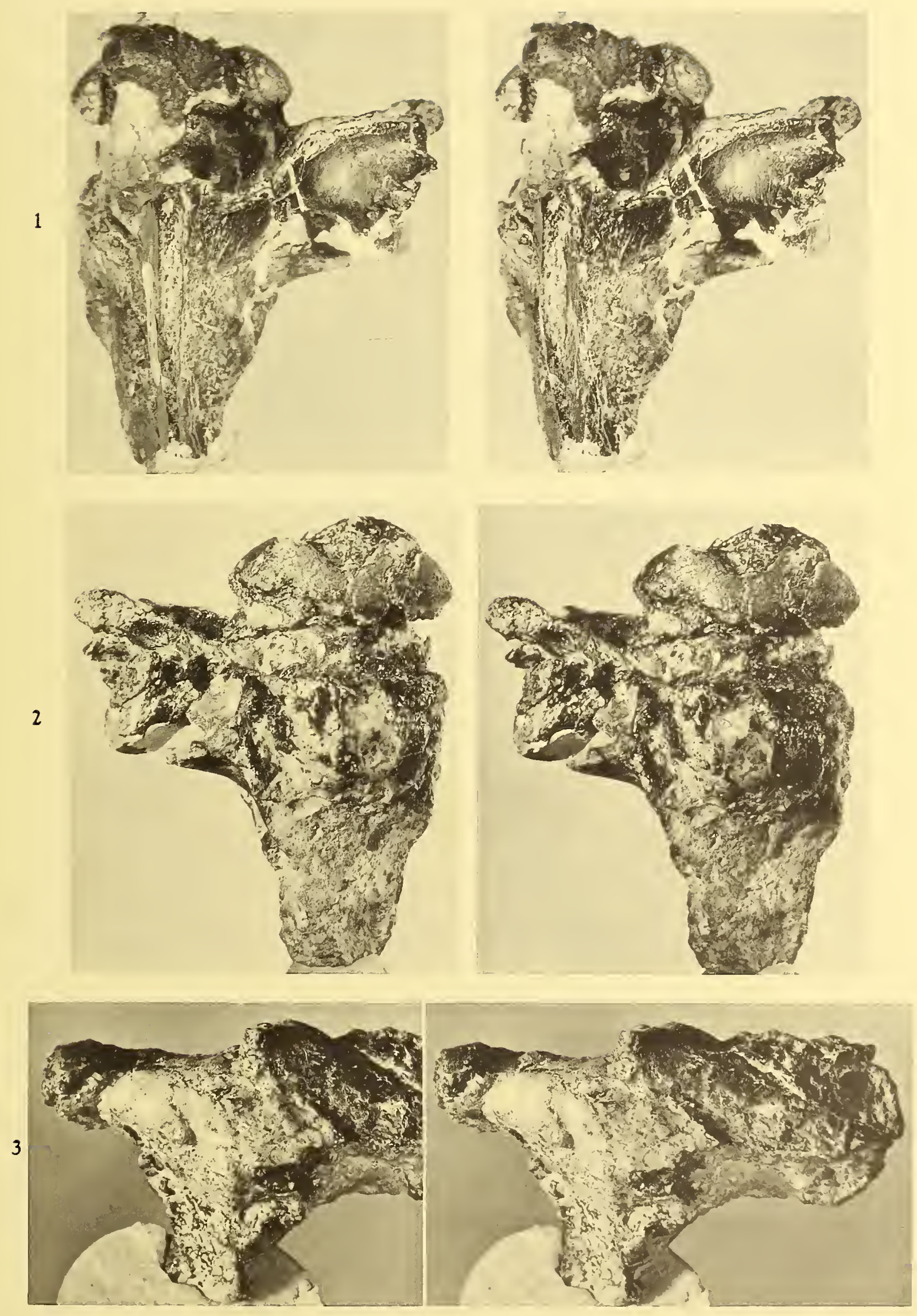



\section{PLATE 9 \\ OLIGOKYPHUS}


FIG, I.--Internal view of left periotic and part of basicranium.

R.7IIO. $\times 3.5$.

FIG. 2.-Ventral view of left periotic and posterior part of basicranium. R.7IIo. $\times 3 \cdot 8$.
FIG. 3.-Dorsal view of basicranium. R.7II3. $\times$ 3. The vascular duct is shown leading from brain through subarcuate fossa and from labyrinth to anterior opening of posttemporal foramen.
FIG. 4.-Lateral view of posterior part of skull. R.7ogo. $\times 3$.
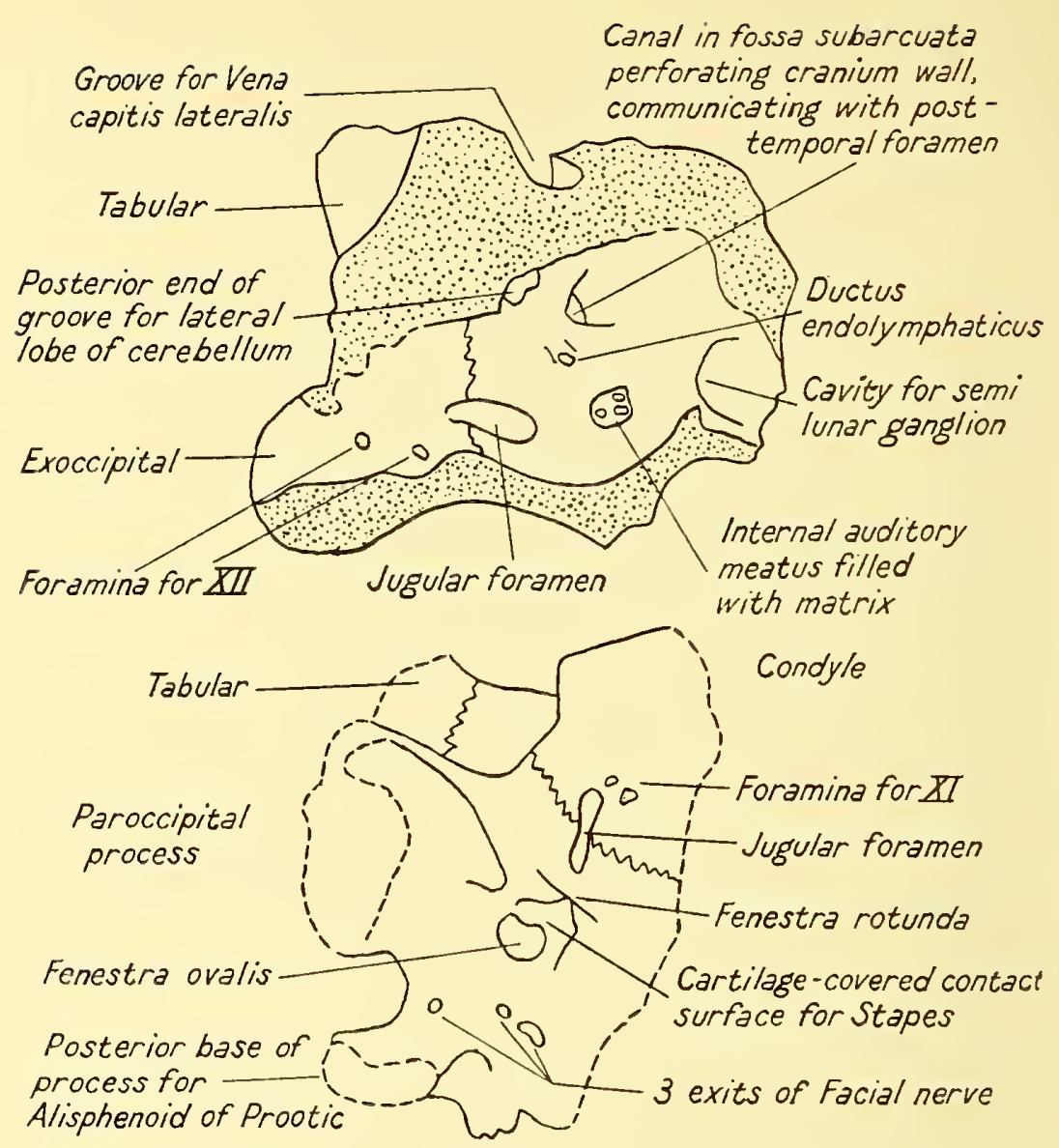

Post temporal foramen

Sutural surface for Squamosal

Tympanic cavity in paroccipital process Vascular duct leading from brain through subarcuate fossa and $\mathrm{AC} C$ from labyrinth to anterior opening of post-temporal foramen

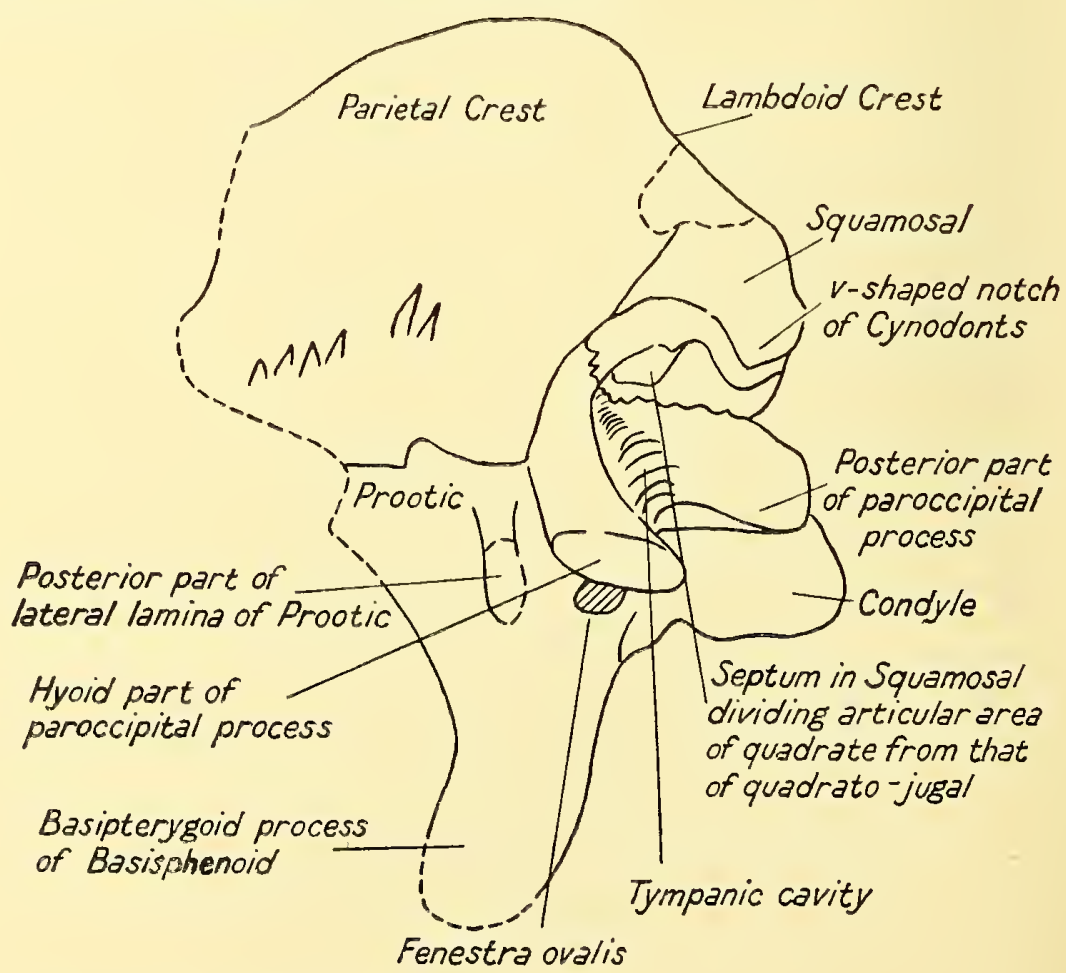

KEY TO PLATE 9 

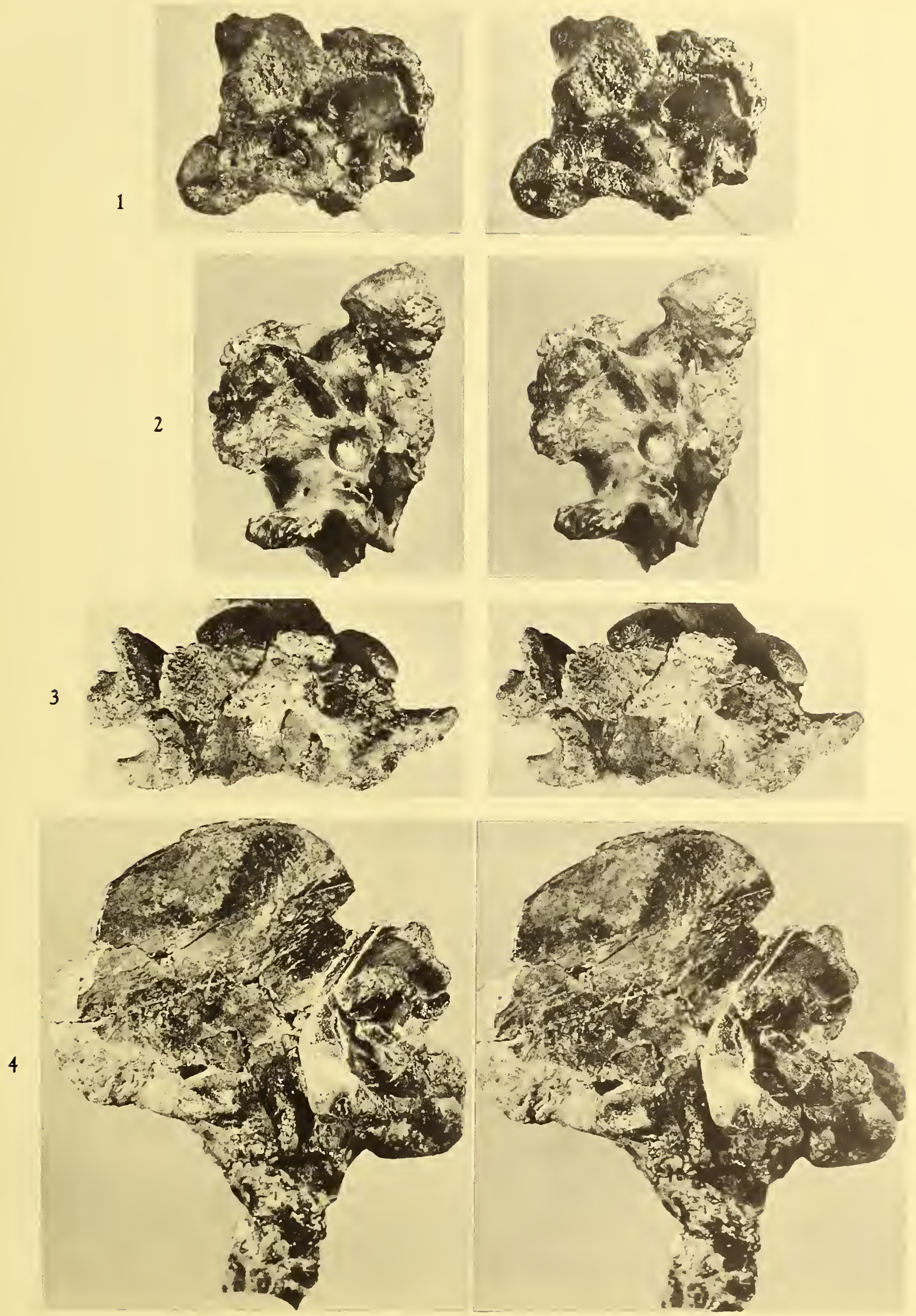

PLATE I O

OLIGOKYPHUS 

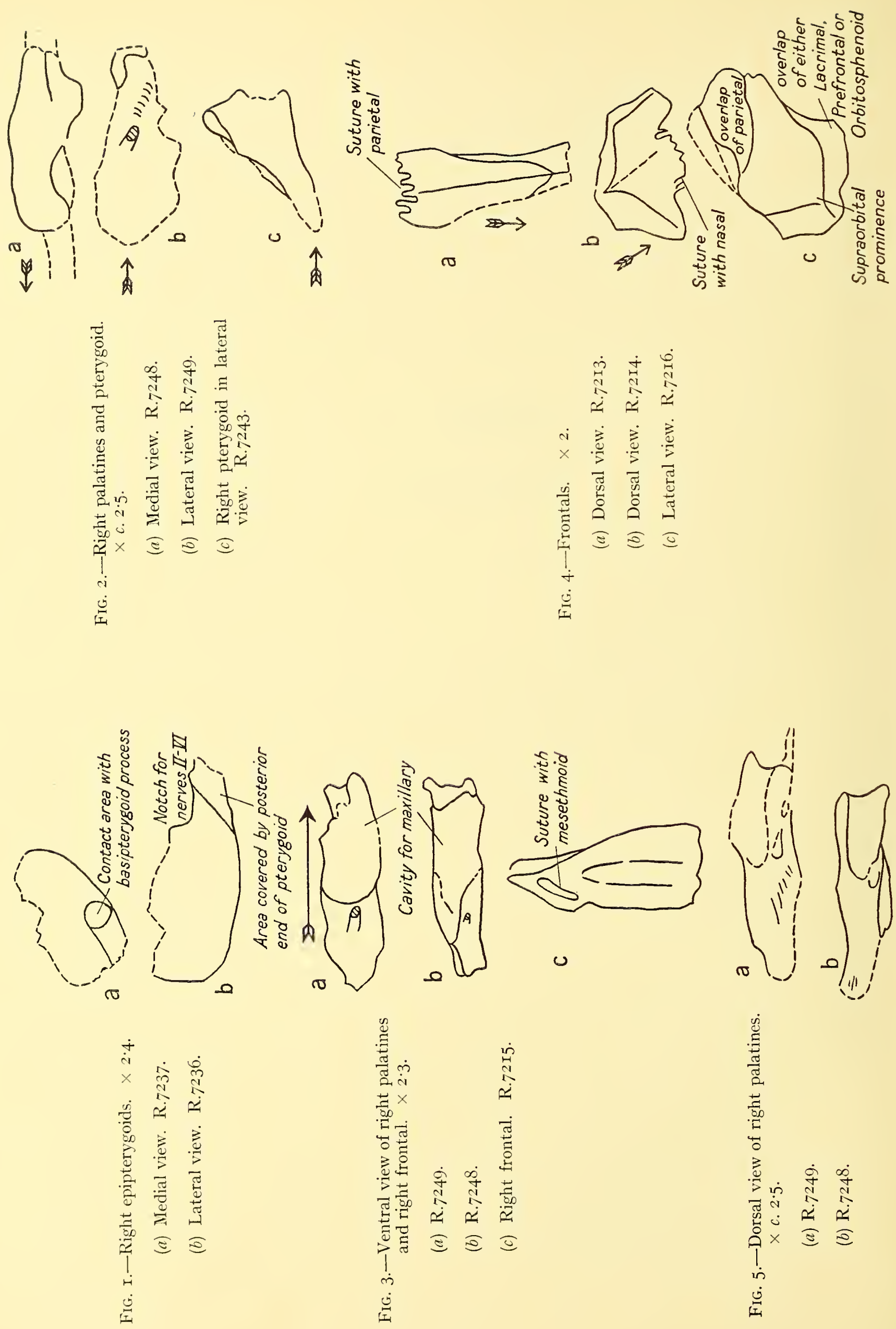

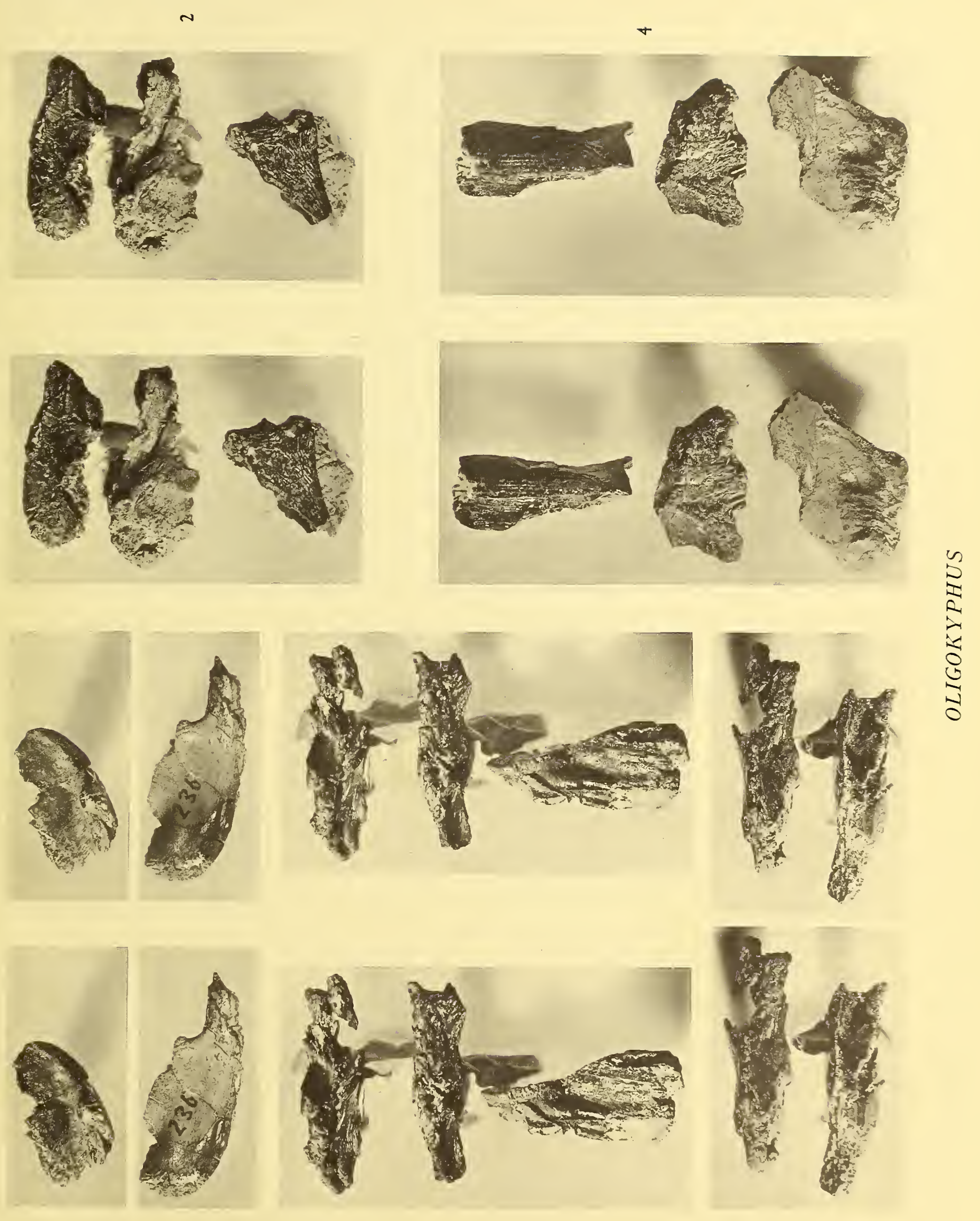

PLATE I I
OLIGOKYPHUS 
FIG. I.-Left maxillaries with cheek teeth I-5.

$\times 2 \cdot 3$.

Left: Type of Oligokyphus minor. R.7025.

Right: Type of Oligokyphus major.

R.7030.
FIG. 2.-Right maxillary. Small specimen with complete dentition. Heavily worn. The anterior part of the first tooth has escaped wear due to loss of its antagonist. R.7254. $\times 2 \cdot 6$.

FIG. 3.-Dentaries. $\times 2 \cdot 6$.

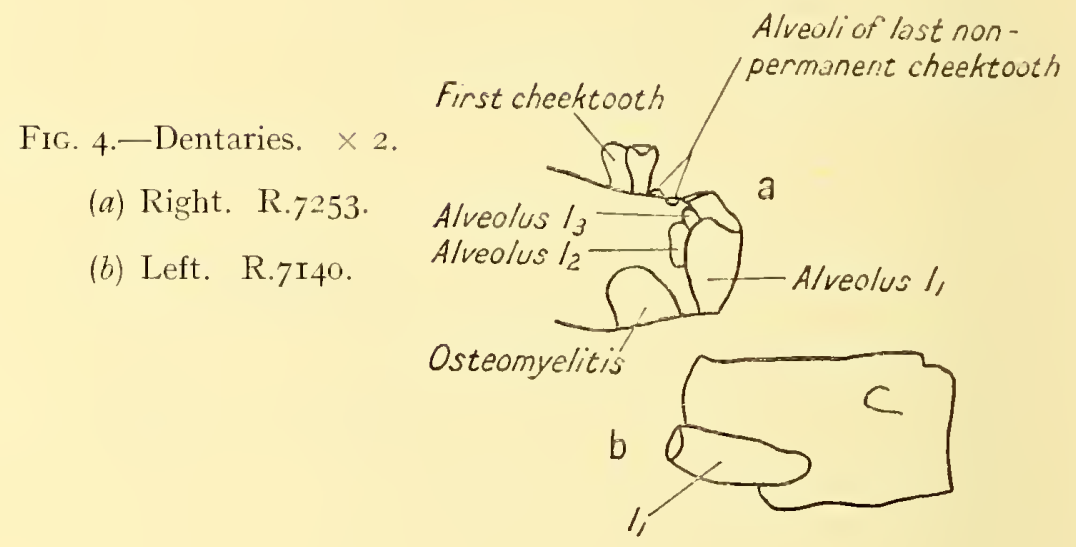

FIG. 5.-Right dentary showing implantation of roots. R.7I80.

FIG. 6.-Maxillary (R.7030) and dentary (R.7I8I) in occlusion. $\times 2 \cdot 2$

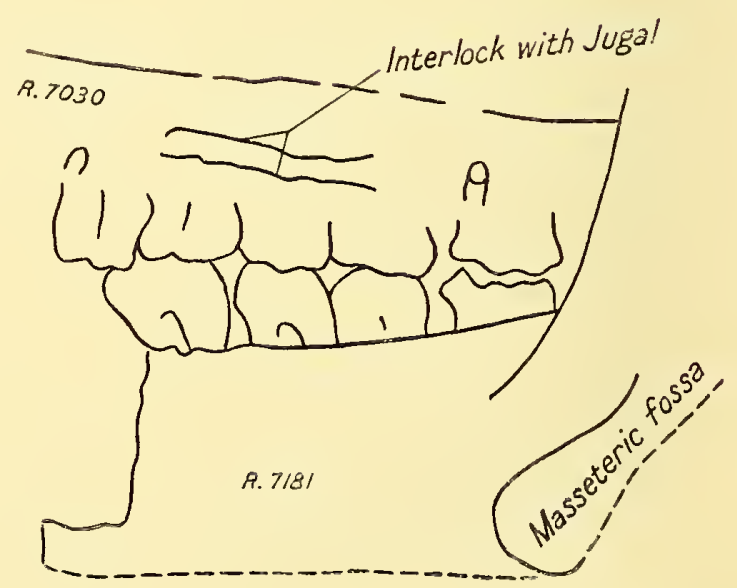


Brit. Mus. (NAt. Hist.)

Plate 11
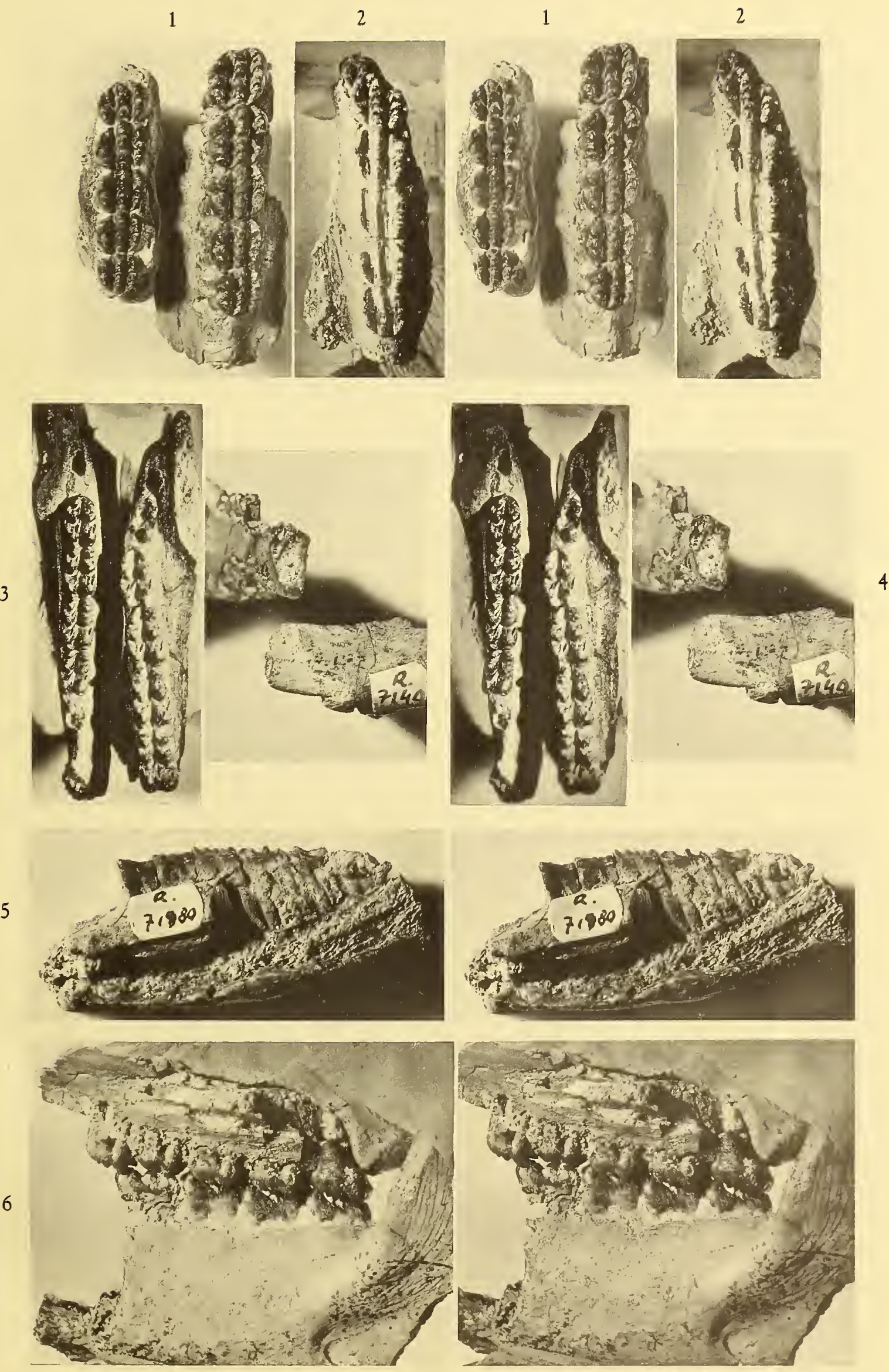

PLATE I 2

OLIGOKYPHUS 

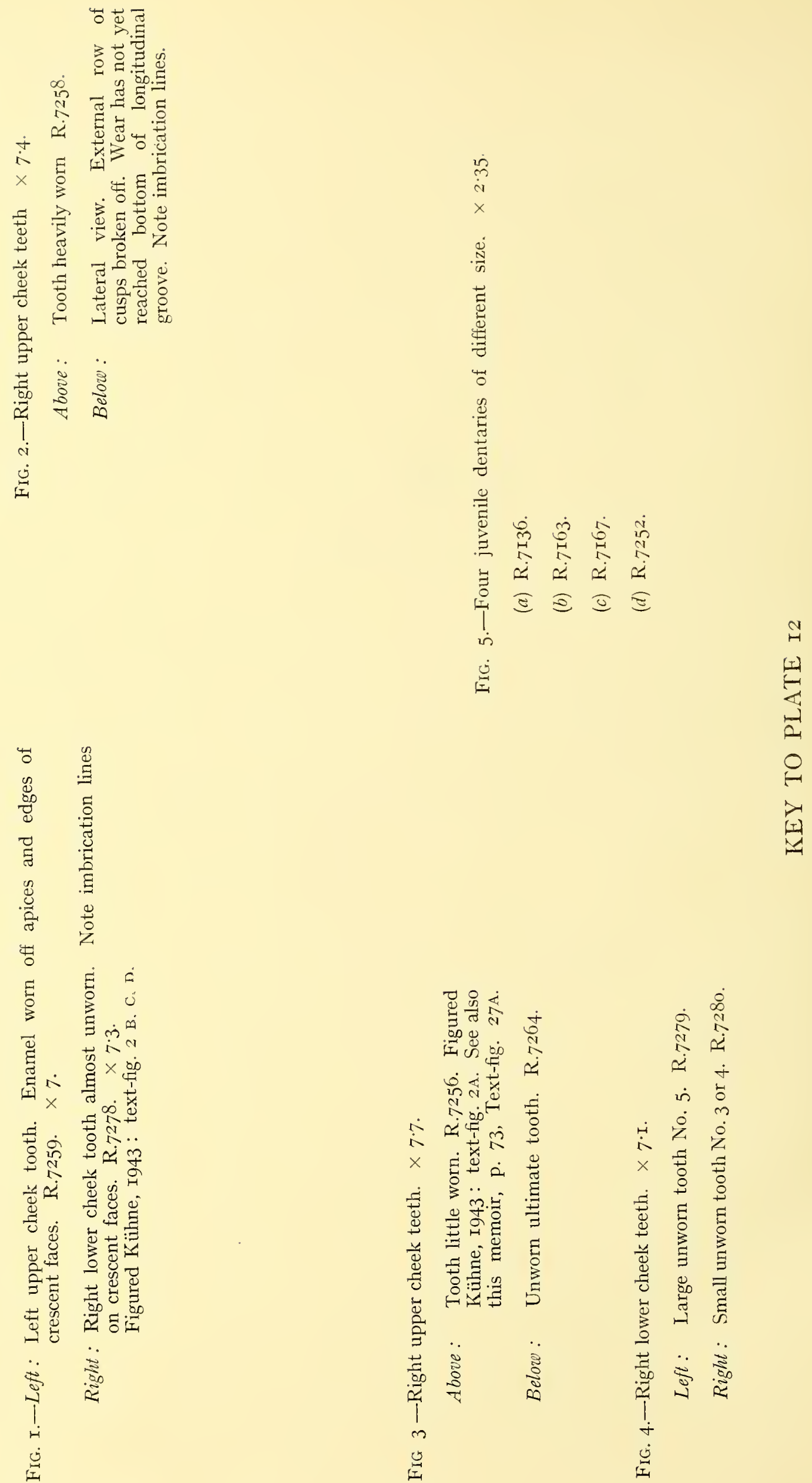

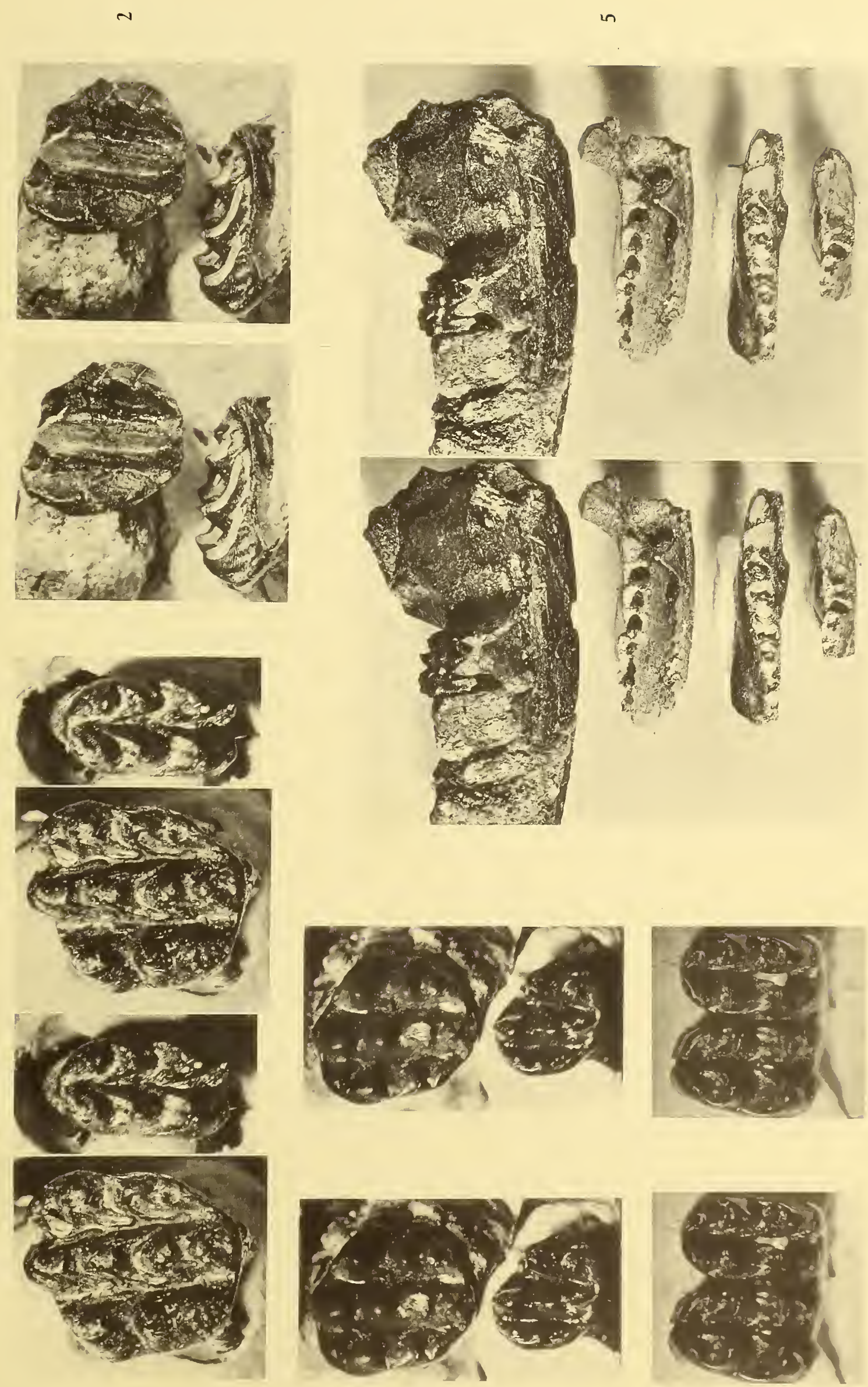

3
0
0
0
0
0
0
0 
$\ominus$ 



